\title{
AGC-3 Graphite Preirradiation Data Analysis Report
}

William Windes

W. David Swank

David Rohrbaugh

Joseph Lord

September 2013

The INL is a

U.S. Department of Energy

National Laboratory

operated by

Battelle Energy Alliance

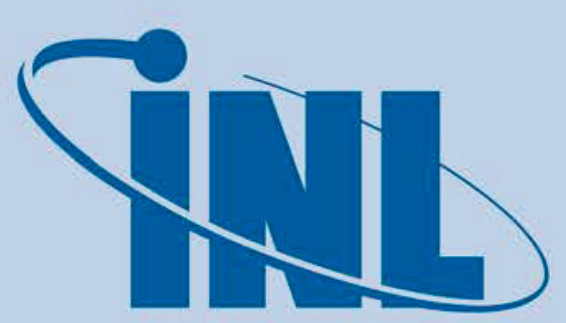

Idaho National Laboratory

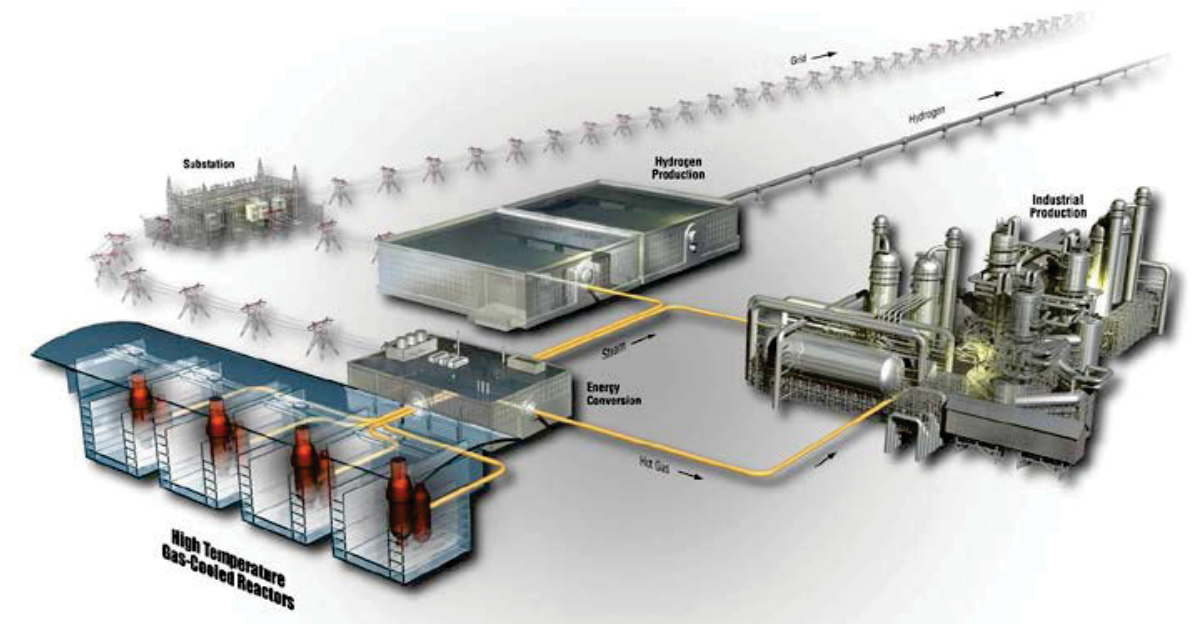




\section{DISCLAIMER}

This information was prepared as an account of work sponsored by an agency of the U.S. Government. Neither the U.S. Government nor any agency thereof, nor any of their employees, makes any warranty, expressed or implied, or assumes any legal liability or responsibility for the accuracy, completeness, or usefulness, of any information, apparatus, product, or process disclosed, or represents that its use would not infringe privately owned rights. References herein to any specific commercial product, process, or service by trade name, trade mark, manufacturer, or otherwise, does not necessarily constitute or imply its endorsement, recommendation, or favoring by the U.S. Government or any agency thereof. The views and opinions of authors expressed herein do not necessarily state or reflect those of the U.S. Government or any agency thereof. 
INL/EXT-13-30297

Revision 0

\section{AGC-3 Graphite Preirradiation Data Analysis Report}

William Windes, W. David Swank, David Rohrbaugh, and Joseph Lord

September 2013

Idaho National Laboratory

VHTR Program

Idaho Falls, Idaho 83415

http://www.inl.gov

Prepared for the

U.S. Department of Energy

Office of Nuclear Energy

Under DOE Idaho Operations Office

Contract DE-AC07-05ID14517 

VHTR Program

\section{AGC-3 Graphite Preirradiation Data Analysis Report}

INL/EXT-13-30297

Revision 0

September 2013

Approved by:

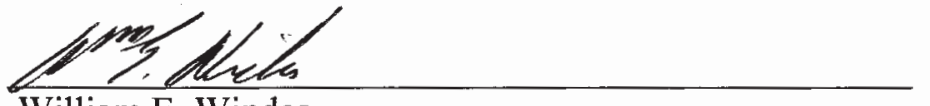

William E. Windes

Author

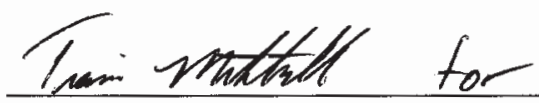

Tim D. Burchell

Independent Technical Reviewer

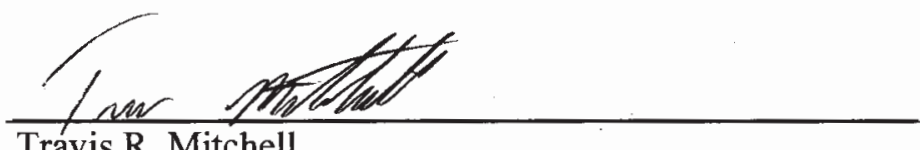

Travis R. Mitchell

VHTR TDO Project Manager

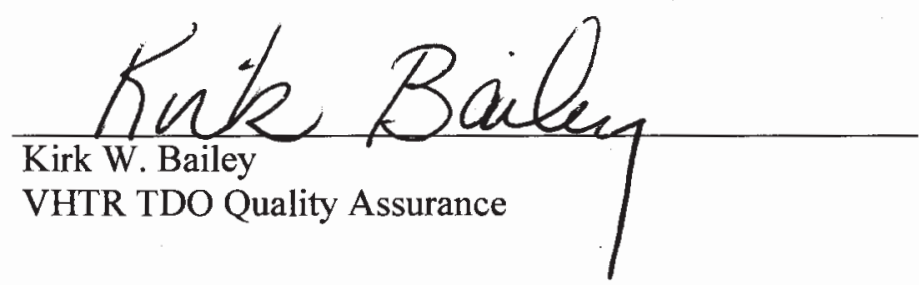

$9 / 30 / 2013$

Date

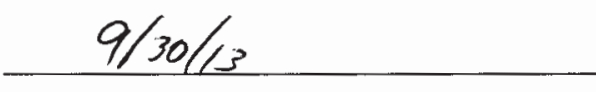

Date

\section{$9 / 30 / 13$}

Date

$9 \cdot 30 \cdot 2013$

Date 



\section{SUMMARY}

This report describes the specimen loading order and documents all preirradiation examination material property measurement data for the graphite specimens contained within the third Advanced Graphite Capsule (AGC-3) irradiation capsule. The AGC-3 capsule is third in six planned irradiation capsules comprising the Advanced Graphite Creep (AGC) test series. The AGC test series is used to irradiate graphite specimens allowing quantitative data necessary for predicting the irradiation behavior and operating performance of new nuclear graphite grades to be generated, which will ascertain the in-service behavior of the graphite for pebble bed and prismatic Very High Temperature Reactor (VHTR) designs. The general design of the AGC-3 test capsule is similar to the AGC-2 test capsule. Material property tests were conducted on graphite specimens prior to loading into the AGC-3 irradiation assembly. However, the six major nuclear graphite grades in AGC-2 were modified, two previous graphite grades (IG-430 and H-451) were eliminated, and one graphite grade was added (Mersen's 2114 was added). Specimen testing from three graphite grades (PCEA, 2114, and NBG-17) was conducted at Idaho National Laboratory (INL) and specimen testing for two grades (IG-110 and NBG-18) was conducted at Oak Ridge National Laboratory (ORNL) from May 2011 to July 2013.

This report also details the specimen loading methodology for the graphite specimens inside the AGC-3 irradiation capsule. The AGC-3 capsule design requires "matched pair" creep specimens that have similar dose levels above and below the neutron flux profile mid-plane to provide similar specimens with and without an applied load. This experiment utilized the neutron flux profile calculated for the AGC-3 capsule design, the capsule dimensions, and the size (length) of the selected graphite and silicon carbide specimens to create a stacking order that can produce "matched pairs" of graphite specimens above and below the AGC-3 capsule elevation mid-point to provide specimens with similar neutron dose levels. 


\section{CONTENTS}

SUMMARY vii

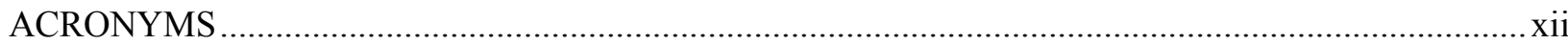

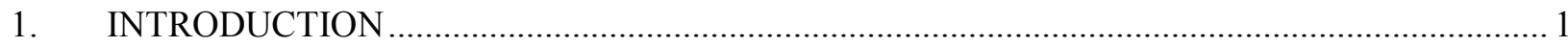

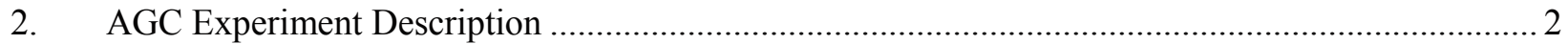

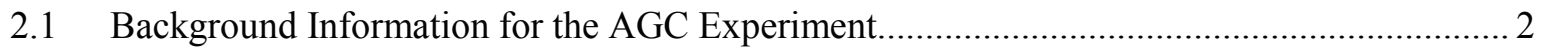

2.2 Description of AGC-3 Test Series .............................................................................. 4

2.2.1 Establishing the Capsule Physical Centerline to the Core Neutron Flux Mid-

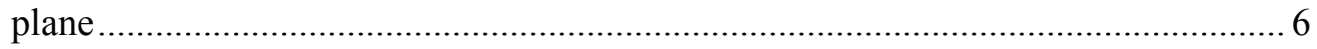

2.2.2 Establishing the Dose Levels as a Function of Position Within the Capsule.............. 8

2.2.3 Determining the Physical Positions of Irradiation Creep Specimens in the

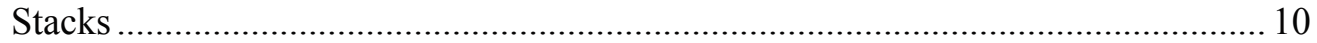

2.2.4 Determining the Physical Positions of Piggy-Back Specimens in the Central

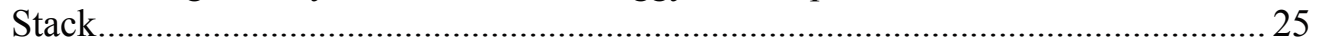

3. PREIRRADIATION MATERIAL PROPERTY MEASUREMENTS …..................................... 25

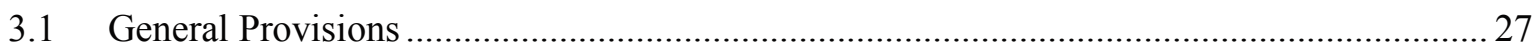

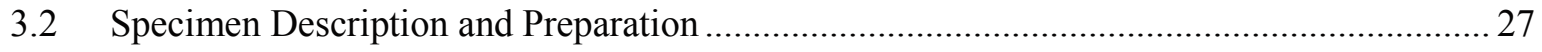

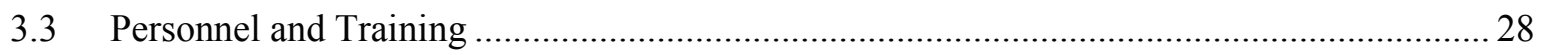

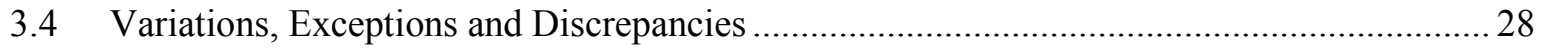

3.5 Calibration and Functional Validation .......................................................................... 29

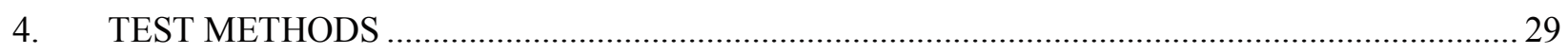

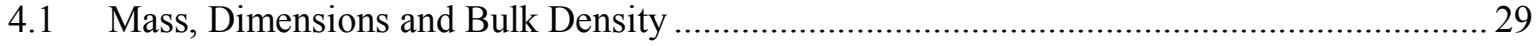

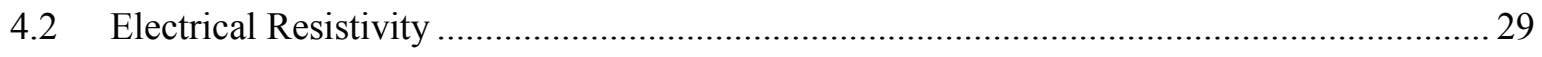

4.3 Approximation of Elastic Modulus from the Measurement of Sonic Velocity .................... 30

4.4 Modulus of Elasticity by Measurement of Fundamental Frequency ..................................... 33

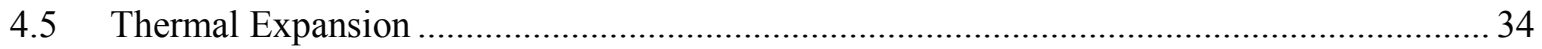

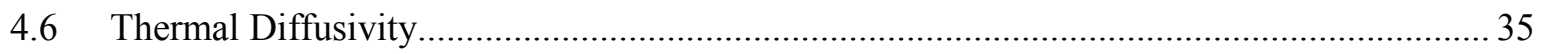

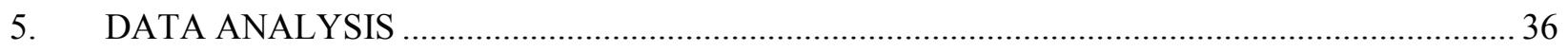

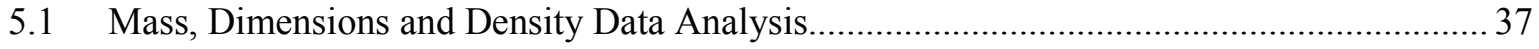

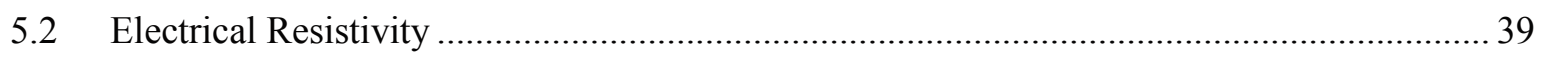

5.3 Approximation of Elastic Modulus from the Measurement of Sonic Velocity .................... 40

5.4 Modulus of Elasticity by Measurement of Fundamental Frequency .................................... 41

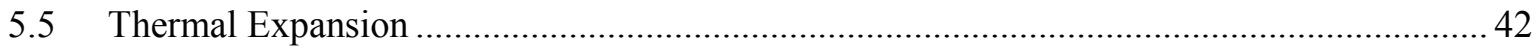

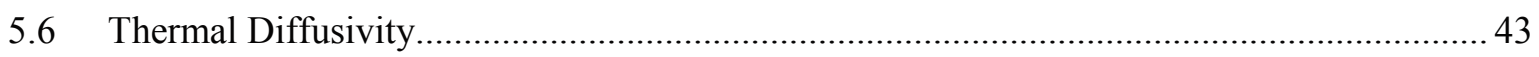

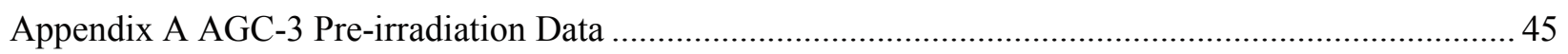

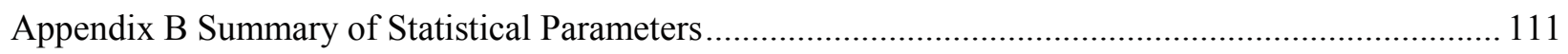




\section{FIGURES}

Figure 1. Original design of AGC experiment illustrating planned dose levels and irradiation temperatures for all six test irradiation capsules.

Figure 2. Revised irradiation dose and temperature parameters of the AGC test series. Note the gray area between AGC-1 and AGC-2 is the expected dose overlap between capsule irradiation.

Figure 3. AGC-3 specimen and capsule elevation locations (from DWG 603524 - AGC-3

Specimen Stack-up Arrangements).

Figure 4. Calculated dose levels for each stack position within AGC-3 after 140 full power days. ............ 8

Figure 5. AGC-3 dose profiles for top and bottom specimens with various offset distances..................... 9

Figure 6. Calculated difference between top and bottom stack dose levels........................................... 10

Figure 7. Typical AGC-3 dose profile for creep graphite specimens utilizing similar applied stress

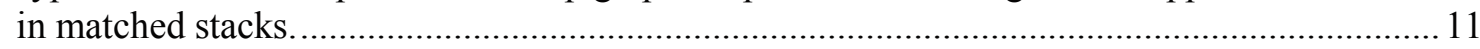

Figure 8. Estimated creep specimen dose profiles for each major graphite type .................................... 14

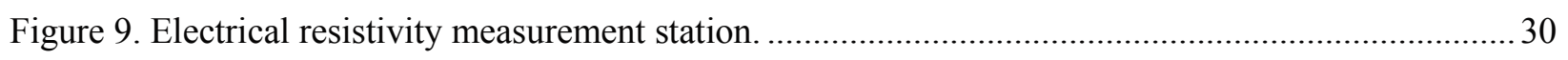

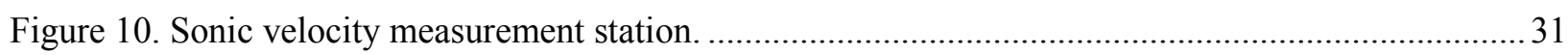

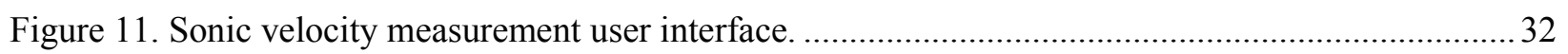

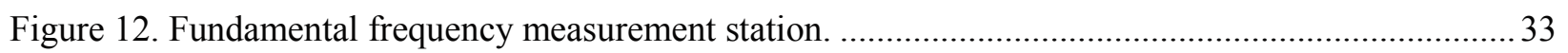

Figure 13. Commercial push rod dilatometer for measurement of CTE.................................................. 35

Figure 14. LFA measurement station for determination of thermal diffusivity......................................... 36

Figure 15. Schematic showing location and dimensional details of NBG-17 billet density

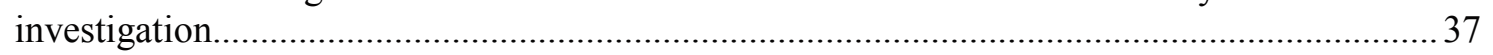

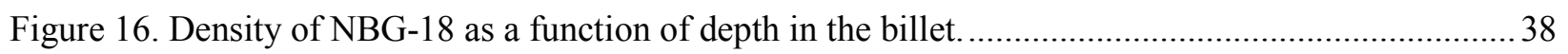

Figure 17. Young's modulus by sonic velocity of NBG-17 as a function of density............................... 38

Figure 18. Electrical resistivity for several nuclear grade graphite types. The anisotropy ratio is called out above each set of data bars.

Figure 19. Mean CTE for several types of nuclear grade graphite as a function of temperature. Error bars represent \pm 1 standard deviation in the data that was used to obtain the averages plotted.

Figure 20. CTE anisotropy ratio for several types of nuclear grade graphite as a function of temperature.

Figure 21. Thermal diffusivity for various graphite types as a function of temperature. Error bars are 1 standard deviation in the data and in some cases smaller than the data symbol. 
Figure 22. Thermal diffusivity anisotropy ratio for several types of nuclear grade graphite as a function of temperature.

\section{TABLES}

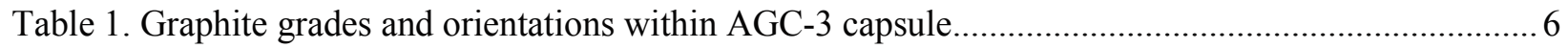

Table 2. Total number of irradiated creep specimens in AGC-3 capsule. .............................................. 11

Table 3. Final loading configuration for AGC-3 creep specimens in outer stacks. ............................... 14

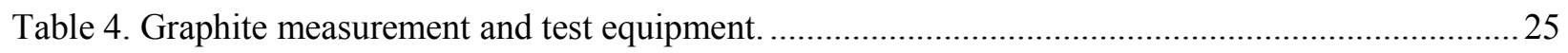

Table 5. Young's modulus by measurement of sonic velocity ................................................................. 40

Table 6. Young's modulus by measurement of fundamental frequency................................................ 41

Table B-1. Creep Pre Thermal Measurement Length (mm) Summary Statistics. .................................. 111

Table B-2. Creep Pre Thermal Measurement Diameter (mm) Summary Statistics................................ 112

Table B-3.Creep Pre Thermal Measurement Mass (g) Summary Statistics. .......................................... 113

Table B-4. Creep Pre Thermal Measurement Density $\left(\mathrm{g} / \mathrm{cm}^{3}\right)$ Summary Statistics. ............................. 114

Table B-5. Creep Coefficient of Thermal Expansion $(1 / \mathrm{K})$ at $100^{\circ} \mathrm{C}$ Summary Statistics..................... 115

Table B-6. Creep Coefficient of Thermal Expansion $(1 / \mathrm{K})$ at $500{ }^{\circ} \mathrm{C}$ Summary Statistics..................... 116

Table B-7. Creep Coefficient of Thermal Expansion $(1 / \mathrm{K})$ at $900{ }^{\circ} \mathrm{C}$ Summary Statistics..................... 117

Table B-8. Creep Modulus (GPa) by Sonic Resonance Summary Statistics........................................ 118

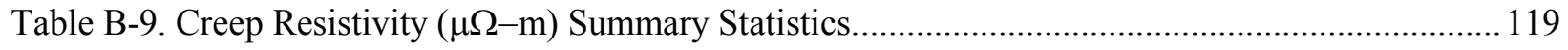

Table B-10. Creep Young's Modulus (GPa) by Sonic Velocity.......................................................... 120

Table B-11. Creep Shear Modulus (GPa) by Sonic Velocity. ............................................................. 121

Table B-12. Piggyback Pre Thermal Measurement Length (mm) Summary Statistics......................... 122

Table B-13. Piggyback Pre Thermal Measurement Diameter (mm) Summary Statistics. ..................... 123

Table B-14. Piggyback Pre Thermal Measurement Mass (g) Summary Statistics................................ 124

Table B-15. Piggyback Pre Thermal Measurement Density $\left(\mathrm{g} / \mathrm{cm}^{3}\right)$ Summary Statistics...................... 125

Table B-16. Piggyback Diffusivity $\left(\mathrm{mm}^{2} / \mathrm{sec}\right)$ at $100{ }^{\circ} \mathrm{C}$ Summary Statistics....................................... 126

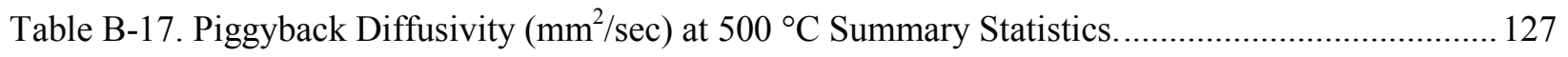

Table B-18. Piggyback Diffusivity $\left(\mathrm{mm}^{2} / \mathrm{sec}\right)$ at $900{ }^{\circ} \mathrm{C}$ Summary Statistics..................................... 128 


\section{ACRONYMS}

\begin{tabular}{|c|c|}
\hline AGC & Advanced Graphite Creep \\
\hline AGC-1 & First Advanced Graphite Capsule \\
\hline AGC-3 & Third Advanced Graphite Capsule \\
\hline ASTM & American Society for Testing and Materials \\
\hline ATR & Advanced Test Reactor \\
\hline $\mathrm{COV}$ & Coefficient of Variation \\
\hline CTE & coefficient of thermal expansion \\
\hline EFPD & Effective Full Power Days \\
\hline HOPG & Highly Oriented Pyrolytic Graphite \\
\hline HTGR & high temperature gas-cooled reactor \\
\hline INL & Idaho National Laboratory \\
\hline IQR & interquartile range \\
\hline LFA & Laser Flash Apparatus \\
\hline LWP & Laboratory-wide Procedure \\
\hline MCP & Management Control Procedure \\
\hline NDMAS & NGNP Data Management and Analysis System \\
\hline NGNP & Next Generation Nuclear Plant \\
\hline ORNL & Oak Ridge National Laboratory \\
\hline PB & Piggy-Back specimen \\
\hline PI & principal investigator \\
\hline PIE & post-irradiation examination \\
\hline PLN & Plan \\
\hline Pre-IE & Preirradiation Examination \\
\hline $\mathrm{R} \& \mathrm{D}$ & research and development \\
\hline TDP & Technology Development Plan \\
\hline VHTR & Very High Temperature Reactor \\
\hline
\end{tabular}




\section{AGC-3 Graphite Preirradiation Data Analysis Report}

\section{INTRODUCTION}

The Next Generation Nuclear Plant (NGNP) will be a helium-cooled, Very High Temperature Reactor (VHTR) with a large graphite core. In past applications, graphite has been used effectively as a structural and moderator material in both research and commercial High Temperature Gas-cooled Reactor (HTGR) designs [1]. Nuclear graphite type H-451, used previously in the United States for nuclear reactor core components, is no longer available. New graphite types have been developed and are considered suitable candidates for the new NGNP reactor design. To support the design and licensing of NGNP core components within a commercial reactor, a complete properties database must be developed for these current grades of graphite. Quantitative data are required for the physical, mechanical, and thermal properties of each graphite type with a specific emphasis on data accounting for the life-limiting effects of irradiation creep on key physical properties of the candidate graphites for NGNP. Further details on the research and development (R\&D) activities and associated rationale needed to qualify nuclear grade graphite for use within the NGNP are documented within the Graphite R\&D Technology Development Plan (TDP) [2, 3].

Based upon previous experience with earlier graphite core components, the phenomenon of irradiation-induced creep within the graphite has been discovered to be critical to the total useful lifetime of the graphite components. Irradiation-induced creep occurs under the simultaneous application of high temperatures, neutron irradiation, and applied stresses within the graphite components. Significant internal stresses within the graphite components can arise from a second phenomenon, irradiation-induced dimensional change, where the graphite physically changes (first shrinking and then expanding if greater levels of irradiation dose are experienced). This disparity in material volume change can induce significant internal stresses within the graphite components. Irradiation-induced creep assists in the removal of these large internal stresses, thus reducing the risk of crack formation and component failure. Obviously, higher irradiation creep levels tend to relieve more internal stress allowing the components longer useful lifetimes within the core. Determining the irradiation creep rates of nuclear graphites is critical for determining the useful lifetime of the graphite components and is a major component of the NGNP Graphite R\&D Program.

To determine the in-service behavior of these new graphite types for both pebble-bed and prismatic reactor designs, the Advanced Graphite Creep (AGC) experiment is underway. This experiment is examining the properties and behavior of nuclear grade graphites over a large spectrum of temperatures, irradiation fluencies, and applied stress levels to induce irradiation creep that is expected within a VHTR graphite component. The irradiation data is provided through the AGC test series, which is comprised of six planned irradiation capsules irradiated in the Advanced Test Reactor (ATR) in a large flux trap [2]. The test series exposes the selected graphite specimens to temperatures and a range of doses that are expected within the VHTR design. Each irradiation capsule consists of over 400 graphite specimens that are characterized before and after irradiation. Six irradiation capsules are planned within the AGC test series in order to cover all VHTR temperature and irradiation conditions (Figure 1).

The first irradiation capsule of the AGC test series, AGC-1, has been initially characterized, loaded into ATR, irradiated and is currently undergoing post-irradiation examination (PIE) $[4,5,6]$. The second irradiation capsule, AGC-2, has been initially characterized, loaded into ATR, irradiated, and is currently undergoing specimen disassembly from the irradiation capsule. Characterization of the graphite specimens for the third capsule (AGC-3) was recently completed and the material property measurements and specimen loading order are reported here. Three graphite grades were changed within AGC-3 to accommodate the requirement for more specimens of selected major grades. H-451 and IG-430 were removed from the test matrix and S2020 was replaced with the vendor's preferred grade 2114 . These are 
the final graphite grades to be tested for all remaining AGC capsules. All other test parameters were unchanged for AGC-3 specimens. All AGC-3 material property testing was conducted at Idaho National Laboratory (INL) and Oak Ridge National Laboratory (ORNL) from July 2009 to August 2010 [7].

To achieve the proper irradiation conditions and applied loads to the creep specimens, an exact specimen loading order is critical. Since irradiation creep is usually determined by the difference in dimensional change occurring within specimens that have an applied load and those that do not, it is assumed that the specimens must have the same irradiation dose and irradiation temperature values. To achieve these similar irradiation conditions for "matched pairs," a careful map of where each specimen resides within the irradiation capsule is required. A detailed analysis of the reactor flux profile to ascertain the dose levels for each specimen, as well as the designed loading configurations within the capsule, are required to guarantee that the matched pairs have the same temperature and dose levels. Details of the specimen loading order, the capsule loading design, the flux profile within the ATR, and the resulting estimated dose profiles for each graphite specimen are discussed for this preirradiation data analysis report for AGC-3.

\section{AGC Experiment Description}

The AGC experiment is designed to establish the safe operating envelope of graphite core components for a VHTR by measuring the irradiated material property changes and behavior of several new nuclear grade graphites over a large range of temperatures, neutron fluencies, and mechanical compressive loads. The experiment consists of three interrelated stages: preirradiation characterization of the graphite specimens, the irradiation test series (designated as six separate irradiation capsules), and PIE of the graphite specimens after irradiation. Separate reports for each distinct stage are prepared after the activity is completed. The preirradiation examination reports detail the total number of graphite types and specimens, the specimen loading configuration to expose all specimens to the entire range of irradiation conditions, and the preirradiation material property testing results. The irradiation test series reports detail the irradiation history of each capsule while in the reactor, noting any changes from the technical and function specifications for each specific test series capsule, and identifying the possible improvements to the next test series capsule design. The PIE reports detail the changes in the specimen material property measurements, compare the results to the preirradiation examination (pre-IE) material property measurements, and analyze the data to assist in determining a credible safe operating envelope for graphite core components in a VHTR design and licensing application.

\subsection{Background Information for the AGC Experiment}

The AGC experiment will provide irradiated material property data for current graphite types available for used within a VHTR design. Due to volume limitations within typical material test reactors (i.e., ATR) only a limited number of specimens can be irradiated - far fewer than can be used in an accurate statistical specimen population analysis. Therefore, the AGC only measures the irradiated material property changes and behavior of relatively few specimens of new nuclear grade graphites over the anticipated operating temperature range, neutron fluences and mechanical loads. The experiment does generate quantitative material property change data (and limited irradiation creep data), which will be used in conjunction with the as-fabricated material property measurement program (Baseline program) to predict the in-service behavior and operating performance of these new nuclear graphite grades for pebble bed and prismatic reactor designs. Changes to key thermal, physical, and mechanical material properties are determined by comparing the material properties of each specimen before irradiation and after irradiation. Differences measured from the irradiation conditions will provide irradiation behavior data in graphite with a specific emphasis on data accounting for the life-limiting effects of irradiation creep on key physical properties of several candidate graphites for NGNP. 
The critical component of the AGC Experiment is the irradiation test series, which irradiates the graphite specimens after pre-IE characterization has been completed. The AGC test series is comprised of six planned irradiation capsules that are irradiated in the ATR in a large flux trap as described in the Graphite TDP [2]. The test series exposes test specimens of the selected nuclear grade graphite types to temperatures and a range of doses that are expected within a HTGR design. Specifically, graphite specimens will be exposed to a fast neutron dose $(\mathrm{E}>0.1 \mathrm{MeV})$ ranging from 1 to $7 \mathrm{dpa}$ and temperatures of $600^{\circ} \mathrm{C}, 900^{\circ} \mathrm{C}$, and $1200^{\circ} \mathrm{C}$ (Figure 1). AGC-3 was designed to be irradiated within the ATR's East Flux Trap rather than the South Flux Trap as previously used with AGC-1 and AGC-2 [8]. Generally, for irradiations within the East Flux Trap, it requires approximately 225 effective full-power days to provide a nominal fast neutron dose range (in graphite) of 0.5 to $4.0 \mathrm{dpa}$. For those capsules needing a 3.5-7.0 dpa dose range, the irradiation capsule (containing the graphite specimens) will be irradiated within the East Flux Trap twice as long, approximately 450 full-power days.

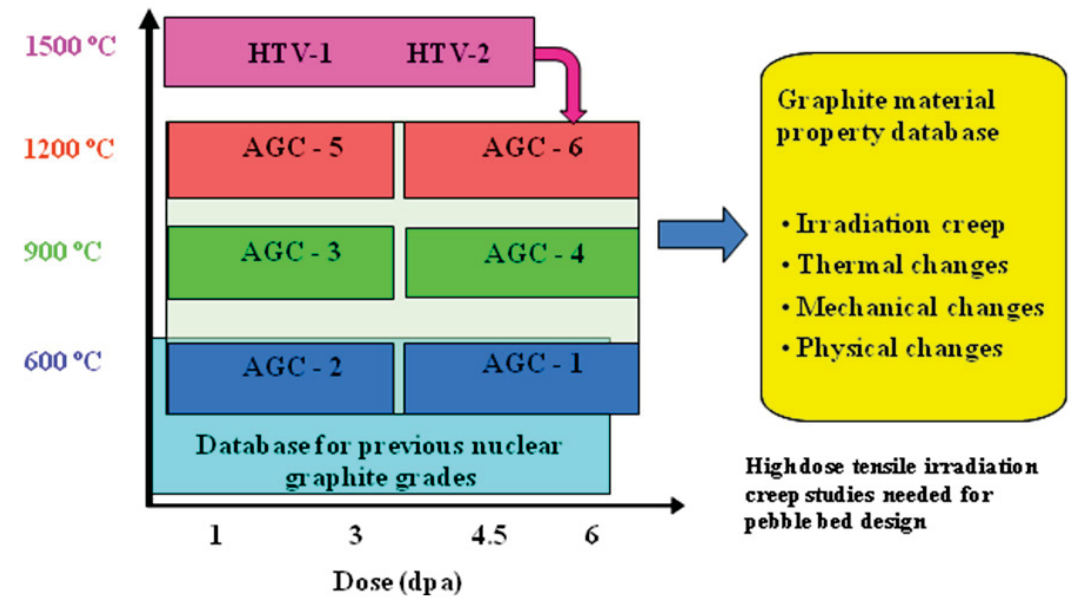

Figure 1. Original design of AGC experiment illustrating planned dose levels and irradiation temperatures for all six test irradiation capsules.

Significant scope is dedicated to determining the irradiation-induced creep rates of the different types of nuclear graphite within the AGC experiment. The traditional method for measuring irradiation-induced creep is to apply a significant load to half the specimens during irradiation while leaving the remaining half of the specimens unloaded. The resulting difference in dimensional change between the loaded and unloaded specimens (assuming the temperature and dose levels are the same) provides the amount of irradiation-induced strain for each "matched pair" of graphite specimens. From this strain level, a creep rate for each graphite type can be calculated as a function of dose if both specimens were irradiated at the same, constant temperature. Thus, each capsule is designed to be irradiated at a constant temperature allowing only the dose and applied mechanical load to vary inside each test series capsule. The creep rate of graphite types is therefore measured as only a function of load and dose within each capsule. To ascertain the temperature dependency of irradiation induced material property changes, the property changes for similar graphite types, at similar dose and load levels, must be compared between capsules. This implies that similar graphite types must be in the same locations in every capsule to receive similar dose and load levels at different temperatures.

Each test series capsule contains two primary specimen types: creep specimens providing irradiation creep rate values as well as mechanical properties and "piggyback" specimens providing thermal material property changes to the graphite. Generally, the creep specimens are larger (25.4 $\mathrm{mm}$ tall) and are irradiated in the mechanically loaded outer stack positions of the capsule body where an applied load can 
be imposed upon half of the specimens. Piggy-back specimens are short (6 $\mathrm{mm}$ tall) button specimens that reside in the axial spine of the capsule receiving no applied load and are subjected only to neutron irradiation to assess the effects of a reactor environment on the specific graphite grade. Together, both types of specimens provide the material property changes for stressed and unstressed graphite types. The physical dimensions for both creep and piggyback specimens are shown in INL Drawing 601501 [9].

The larger creep specimens were best suited for physical and mechanical testing techniques, such as dimensional change, irradiation creep, elastic modulus, density, and thermal expansion. The smaller piggyback specimens were best suited for thermal and physical testing, such as thermal diffusivity, mass measurements, and density. Because piggyback specimens were not stressed during irradiation, new "experimental" types of graphite and graphitic material were allowed to be tested for irradiation stability. These newer experimental types of graphite may provide superior irradiation performance and could be used in future reactor designs.

\subsection{Description of AGC-3 Test Series}

The Advanced Graphite Creep experiment consists of six irradiation capsules irradiating approximately 500 graphite specimens in each capsule. The first two capsules are intended to be irradiated at $600^{\circ} \mathrm{C}$, the next two capsules at $900^{\circ} \mathrm{C}$, and the final two at $1200^{\circ} \mathrm{C}$. The design of these capsules make use of the symmetric neutron flux profile of the ATR to irradiated "matched pairs" of graphite specimens; graphite specimens above the reactor mid-point elevation that are mechanically loaded and matching graphite specimens below the reactor mid-point line that are not mechanically loaded during irradiation. The design of the capsule ensures a constant temperature for all specimens throughout the capsule, but to ensure that specimen "matched pairs" are similar in dose, the specimen stacking order must be matched to the ATR flux profile so that similar specimens on the top portion of the capsule match the dose received in the bottom of the capsule.

To achieve this similar dose level in the AGC-3 test capsule, the ATR flux profile is compared to the specimen/capsule elevation specifications. By taking into account the flux profile, the AGC-3 specimens can be stacked/ordered so that matched pairs will receive similar dose levels on the bottom and top sections of the capsule. This is further complicated by the fact that the axial ATR neutron flux profile is not completely symmetric due to core components altering the profile. This requires a small offset in the specimen stacking order in the bottom half of the capsule to line up specimens to the correct dose level.

In addition, due to the limited volume in the capsules and the large number of graphite grades being investigated, the stacking order must ensure an equal distribution of specimens over the entire dose range in the irradiation capsule. With five graphite grades, three different mechanical load levels, and specimens $25.4 \mathrm{~mm}$ long being investigated, this requires a careful plan to evenly distribute as many specimens as possible over the entire dose range. For this study, some graphite grades were allowed more specimens than other types (i.e., new PCEA graphite verses established Mersen's 2114 type) skewing the number of specimens slightly from grade to grade.

The microstructural orientation of the graphite specimens to the applied load during irradiation is important since it is anticipated that with grain creep rates differ from against-grain creep rates. The microstructure orientation is an additional parameter that must be considered when ordering the specimen stacks.

Finally, each capsule is designed to irradiate all specimens at a constant temperature allowing only the dose and applied mechanical load to vary inside each capsule. Therefore, the creep rate of graphite types is measured as only a function of load and dose within each capsule. To ascertain the temperature dependency of irradiation induced material property changes the creep rates of similar graphite type specimens, at similar dose and load levels, must be compared between capsules. This implies that similar graphite grades must be in the same locations in every capsule to receive similar dose and load levels at 
different temperatures. Since two of the five graphite grades were changed from AGC-1 and 2 to AGC-3, this required performing a check to ensure the specimen locations within AGC-3 matched with similar graphite-type specimens in AGC-2 if applicable.

AGC-3 was originally designed to be the shorter $900^{\circ} \mathrm{C}$ irradiation capsule to provide the 0.5 to 4.0 dpa dose level irradiated specimens, Figure 1. The AGC-3 specimens were planned to be compared to AGC-1 specimens of the same grade experiencing the same stress levels and dose but a tower $600^{\circ} \mathrm{C}$ temperature. However, due to issues with temperature control within the first AGC-1 capsule [5], it was decided during AGC-1 irradiation to hold the capsule inside the ATR as long as possible to ascertain the viability of the capsule design at high dose levels. Therefore, the irradiation dose levels for AGC-1 were changed to the long, 3.5 to $7.0 \mathrm{dpa}$, irradiation levels at the $600^{\circ} \mathrm{C}$ temperature conditions.

Since the AGC-1 specimen temperature limits exceeded the technical specifications, it was determined that it would likely prove difficult to determine material property changes and creep rates at a constant temperature. Therefore, an intermediate, 1.5 to $5 \mathrm{dpa}$, dose level irradiated at a temperature of $600^{\circ} \mathrm{C}$ was selected as the final irradiation conditions for the AGC-2 test series to bridge between the two original $600^{\circ} \mathrm{C}$ test series capsules (Figure 2). It was assumed that some of the specimens from AGC-1 could be utilized in conjunction with the AGC-3 graphite specimens to produce more reliable material property changes at the constant $600^{\circ} \mathrm{C}$ temperature condition. While not optimal, it was determined that providing graphite specimens irradiated over this overlapping dose level at a more consistent temperature condition would be sufficient to ascertain the irradiation creep and material property changes for the selected graphites. No change to irradiation temperature or maximum dose is planned for AGC-3 but the material property comparisons will be with AGC-2 specimens rather than the originally planned AGC-1 specimens.

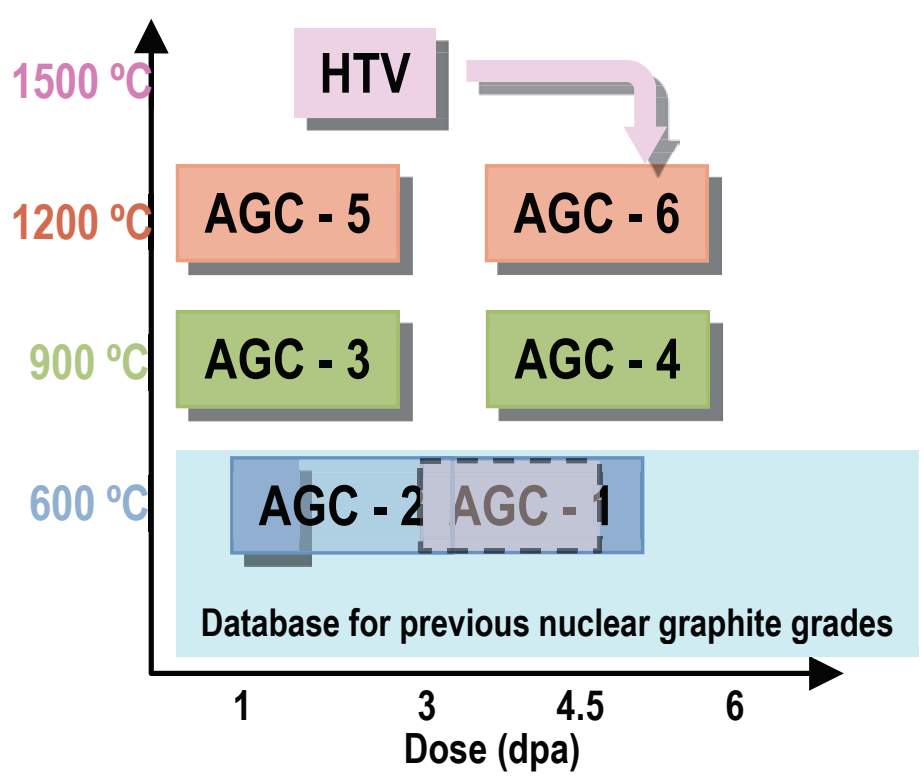

Figure 2. Revised irradiation dose and temperature parameters of the AGC test series. Note the gray area between AGC-1 and AGC-2 is the expected dose overlap between capsule irradiation.

Three of the previous major grades of graphite contained within AGC-1 and AGC-2 were changed. H-451 and IG-430 were removed from the test matrix to allow for more major grade specimen numbers. Additionally, specimens of the preferred nuclear grade 2114 from Mersen USA, which replaced grade S2020 in AGC-2, were added as a major grade. The five remaining major grades of graphite are PCEA, 
NBG-17, NBG-18, IG-110, and 2114. These are the final graphite major grades to be tested for all remaining AGC capsules.

AGC-3 piggyback specimens consisted of small button specimens $(\varnothing 12 \mathrm{~mm} \times 6 \mathrm{~mm})$ of the major grades of graphite plus two graphite container-type specimens: $12.5 \mathrm{~mm}(1 / 2 \mathrm{inch})$ and $6 \mathrm{~mm}(1 / 4 \mathrm{inch})$ tall containers. The $6 \mathrm{~mm}$ (1/4 inch) tall containers each held one Highly Oriented Pyrolytic Graphite (HOPG) specimen. The $12.5 \mathrm{~mm}(1 / 2 \mathrm{inch})$ tall containers each held a silicon carbide-coated graphite hemisphere specimen fabricated at SGL to test for irradiation performance of SiC-coated graphite). Similar to the AGC-1 and AGC-2 designs, piggyback graphite grades were irradiated in the unloaded center stack of AGC-3. A list of all major graphite grades, piggyback grades, and experimental graphite grades is in Table 1 .

Table 1. Graphite grades and orientations within AGC-3 capsule.

\begin{tabular}{|c|c|c|c|}
\hline Grade & Specimen Type & Dimension & $\begin{array}{c}\text { With Grain (wg) - Against Grain } \\
\text { (ag) }\end{array}$ \\
\hline PCEA & Creep & $\varnothing 12.5 \times 25.4 \mathrm{~mm}$ & $36 \mathrm{wg} / 12 \mathrm{ag}$ \\
\hline NBG-17 & Creep & $\varnothing 12.5 \times 25.4 \mathrm{~mm}$ & $18 \mathrm{wg}-18 \mathrm{ag}$ \\
\hline NBG-18 & Creep & $\varnothing 12.5 \times 25.4 \mathrm{~mm}$ & $24 \mathrm{wg}-24 \mathrm{ag}$ \\
\hline 2114 & Creep & $\varnothing 12.5 \times 25.4 \mathrm{~mm}$ & $48 \mathrm{ag}$ \\
\hline IG-110 & Creep & $\emptyset 12.5 \times 25.4 \mathrm{~mm}$ & $32 \mathrm{wg}-14 \mathrm{ag}$ \\
\hline PCEA & Piggyback & $\varnothing 12.5 \times 6.3 \mathrm{~mm}$ & $11 \mathrm{wg}-22 \mathrm{ag}$ \\
\hline NBG-17 & Piggyback & $\varnothing 12.5 \times 6.3 \mathrm{~mm}$ & $21 \mathrm{wg}-9 \mathrm{ag}$ \\
\hline NBG-18 & Piggyback & $\varnothing 12.5 \times 6.3 \mathrm{~mm}$ & $11 \mathrm{wg}-9 \mathrm{ag}$ \\
\hline 2114 & Piggyback & $\varnothing 12.5 \times 6.3 \mathrm{~mm}$ & $24 \mathrm{ag}$ \\
\hline IG-110 & Piggyback & $\varnothing 12.5 \times 6.3 \mathrm{~mm}$ & $17 \mathrm{wg}-12 \mathrm{ag}$ \\
\hline PCIB & Piggyback & $\varnothing 12.5 \times 6.3 \mathrm{~mm}$ & 3 (no direction ID) \\
\hline GrafTech 200 & Piggyback & $\varnothing 12.5 \times 6.3 \mathrm{~mm}$ & 14 (no direction ID) \\
\hline GrafTech 324 & Piggyback & $\varnothing 12.5 \times 6.3 \mathrm{~mm}$ & 9 (no direction ID) \\
\hline GrafTech 325 & Piggyback & $\varnothing 12.5 \times 6.3 \mathrm{~mm}$ & 14 (no direction ID) \\
\hline GrafTech 328 & Piggyback & $\varnothing 12.5 \times 6.3 \mathrm{~mm}$ & 9 (no direction ID) \\
\hline SGL SiC & Piggyback & $\varnothing 12.5 \times 6.3 \mathrm{~mm}$ & 8 (no direction ID) \\
\hline SGL-17 & Piggyback & $\varnothing 12.5 \times 6.3 \mathrm{~mm}$ & 8 (no direction ID) \\
\hline MLRF-1 & 1/2-in. container & $10 \mathrm{~mm}$ hemisphere & 9 (no direction ID) \\
\hline HOPG & 1/4-in. container & $2 \times 2 \times 5 \mathrm{~mm}$ wafer & 17 (NA) \\
\hline
\end{tabular}

\subsubsection{Establishing the Capsule Physical Centerline to the Core Neutron Flux Mid-plane}

To ensure similar dose levels within matched pairs the specimen positions were correlated through the total capsule elevation within the core. Capsule arrangement drawings were used to determine the position of each specimen in the capsule as a function of height above and below the mid-plane of the core neutron flux profile (Figure 3) $[10,11,12]$. The size of each creep specimen, the need for periodically placed spacers containing flux wires, and the space requirements in the top of the stacks for the push rods 
were considered. The core flux mid-plane in relation to the capsule arrangement was established so that the reactor neutron flux field could be correlated to the physical elevations/positions in the capsule. The dose levels through all elevations in the ATR are calculated based upon the core mid-plane flux levels. By establishing the specimen locations to the core mid-plane elevation, each specimen dose below and above the core mid-plane can be estimated.
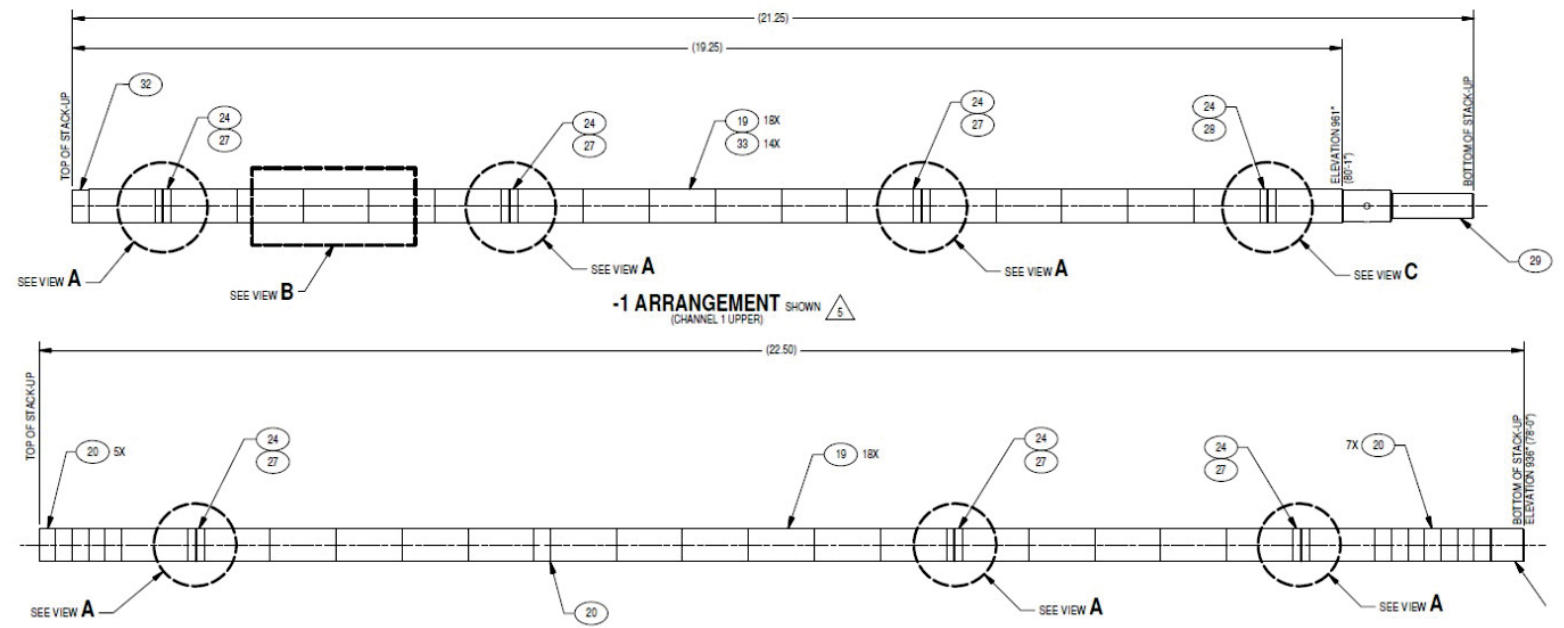

-2 ARRANGEMENT HOWN $\$$

Figure 3. AGC-3 specimen and capsule elevation locations (from DWG 603524 - AGC-3 Specimen Stack-up Arrangements).

Two important points were learned from AGC-1 irradiations: (1) the lower half of the capsule crept under irradiation and produced a slightly smaller gap between the lower stack and the shuttle piston at the end of irradiations, and (2) the loaded specimens in the upper stacks shrink under irradiation substantially more than expected requiring longer push rods for the expected higher levels of shrinkage for this higher temperature capsule. The shuttle piston is used to upset the upper specimens in each of the loaded upper stacks to make sure they do not become bound within the stack during loading. The gap between the shuttle piston and the top of the lower stack is needed to allow free movement of the shuttle piston when it is not engaged. If this gap had been reduced completely, AGC-1 might have transferred the load in the upper stacks to the specimens in the lower stacks. This did not occur, but design changes to the specimen loading to increase the gap were taken in AGC-3 to ensure this did not occur over the expected higher creep rates of AGC-3. Because the upper specimens did not volumetrically shrink the same amount as the lower specimens, the received dose levels did not match the expected values established at the start of the irradiation. The changes are slight, no more than $4 \%$, but this was considered when the final specimen offset distance was established at the beginning of the experiment.

These irradiation lessons learned have a bearing on the final positions of both the upper specimen position and the lower specimen position since the centerlines for all specimens will change over time and irradiation. The centerline movement of the specimens cannot be adjusted for during irradiations. Any final differences between the matched pairs will be accounted for in the post-irradiation analysis of this test series. 


\subsubsection{Establishing the Dose Levels as a Function of Position Within the Capsule}

Once the physical elevation dimensions were established and correlated to the reactor core centerline the dose as a function of distance from the core centerline was determined. The estimated AGC-3 experiment fluence profiles were calculated by Reactor Engineering utilizing a Monte Carlo N-Particle Transport Code model based upon the known neutron flux profile of the ATR, the position of the experiment in the East Flux Trap, and the number of Effective Full Power Days (EFPD) planned for the test series capsule. Irradiation dose values, as a function of distance from the reactor core centerline, were calculated from the total estimated fluence using standard conversion factors for carbon in a fast neutron irradiation field ( $\mathrm{E}>0.1 \mathrm{Mev}$ ) (Figure 4) [13]. As can be seen from Figure 4, after the capsule has been rotated 180 degrees at the irradiation, mid-point the fluence/dose levels for all stacks are shown to be very similar. This indicates a uniform neutron fluence profile for all stacks regardless of their position within the capsule. Since all stacks within the capsule are estimated to have similar dose profiles after complete irradiation the dose profile for the center stack within the capsule was utilized to determine the proper stacking order and specimen offset position in the lower half of the irradiation capsule.

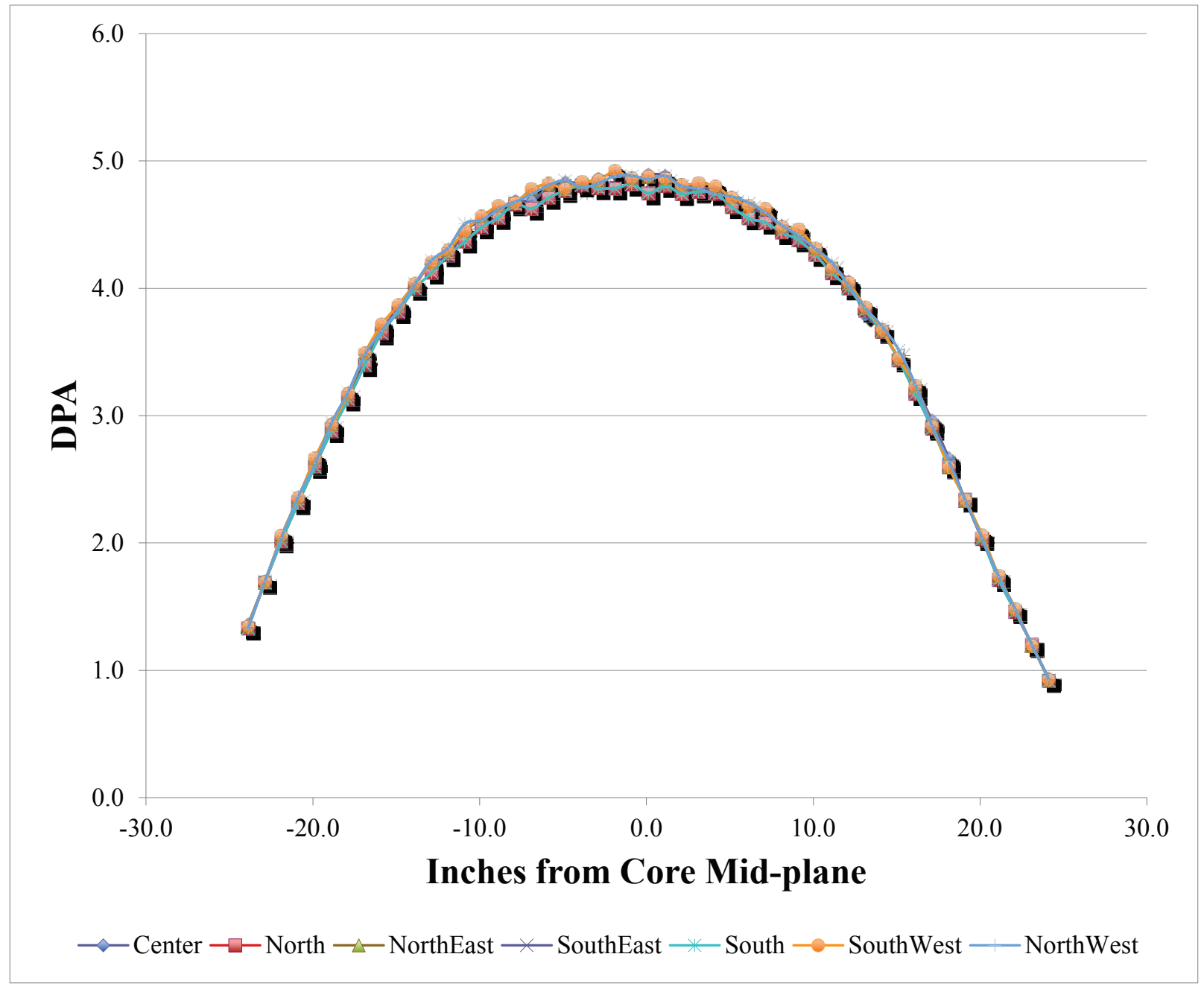

Figure 4. Calculated dose levels for each stack position within AGC-3 after 140 full power days. 
Figure 4 also shows that the ATR neutron flux profile is not completely symmetric along the vertical axis. Thus, to produce matched pair specimens that have similar dose profiles both above and below the core mid-plane, an offset from the mid-plane is required to adjust the specimen positions and match dose levels. For AGC-3, the offset spacing was determined using the accumulated dose levels from the central stack dose profile, both below and above the core mid-plane. Two methods were used to estimate the specimen position offset value: (1) dose levels at $1 / 4$-inch increments (the height of a piggyback specimen) were established from a polynomial fit equation of the curve generated from the dose/position calculations, and (2) "manual" calculations of the average dose values at $1 / 4$-inch increments for each creep specimen were estimated in an Excel spreadsheet.

To determine the length of the offset space, the dose levels calculated at the respective elevations in the AGC-3 capsule were compared as the specimens in the bottom half of the AGC capsule were moved farther away from the mid-plane. The qualitative curves in Figure 5 illustrate how the lower specimen dose profiles change as the specimens are shifted away from the core mid-plane elevation. Particular attention is placed on matching the irradiation dose profiles within the center region of the capsule where the creep specimens were to be irradiated (approximately 38 inches centered on the mid-plane elevation).

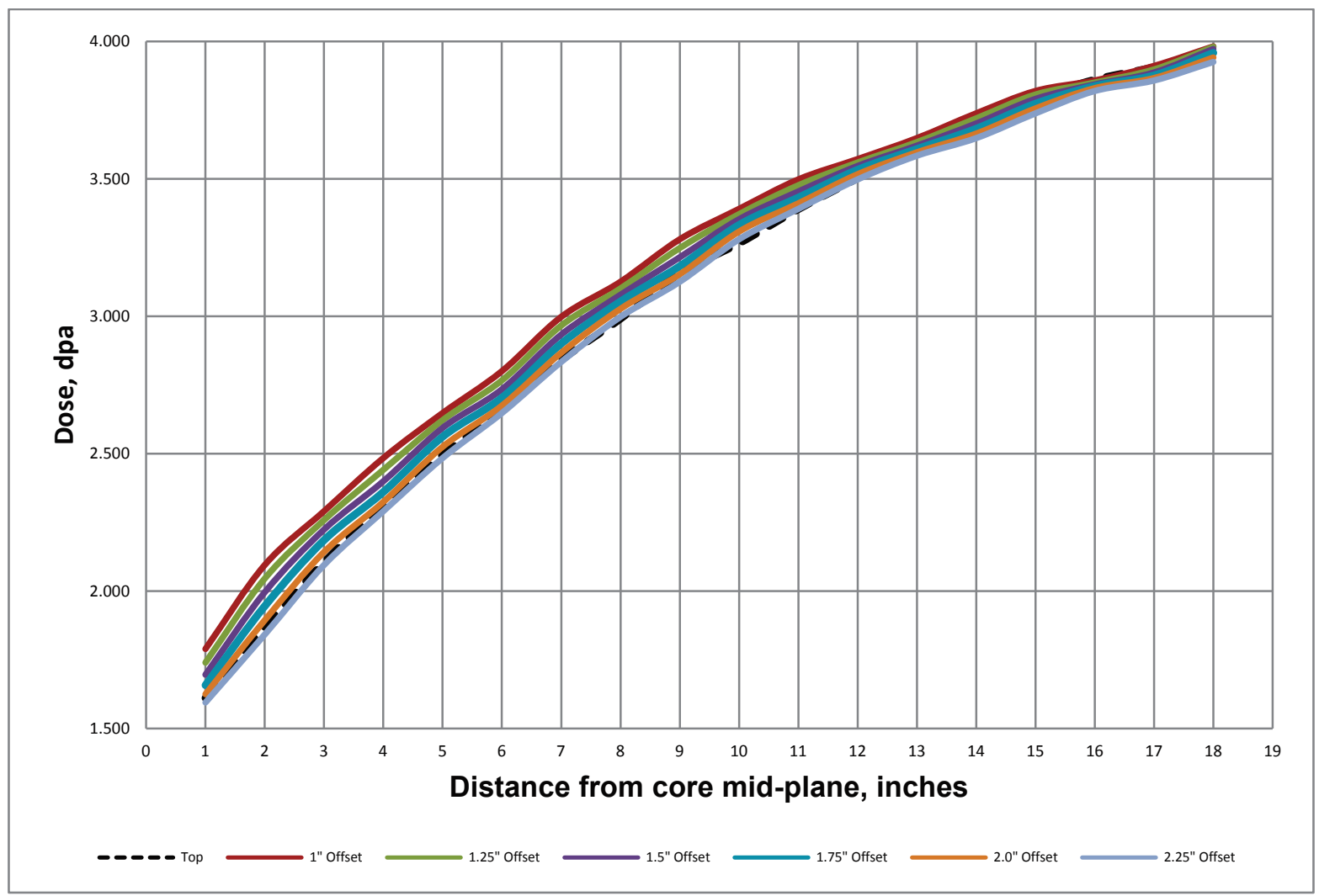

Figure 5. AGC-3 dose profiles for top and bottom specimens with various offset distances.

A more quantitative plot of the differences between calculated dose levels between the top and bottom specimens illustrates the differences between upper and lower specimen matched pair dose profiles (Figure 6). It can be seen that a 1.75-in. offset distance from the mid-plane for the bottom creep specimens produced the closest dose matches between specimens. This offset distance is further warranted when the specimen shrinkage and the graphite specimen holder dimensionally shrinks under irradiation. As seen in both Figure 4 and Figure 5, the dose profiles for the specimen farthest from the core mid-plane have the largest difference between received dose levels. As the upper stack shrinks and 
becomes closer to the core mid-plane this difference is reduced and the dose levels actually become a closer match than was achieved before the irradiation. Effectively, over time and dose the irradiation profiles will become closer due to irradiation shrinkage. While it was impossible to exactly match both the upper and lower matched pair of specimens, the dose levels for each specimen pair where fairly close ranging from 0 to $4 \%$ and converging rather than diverging during irradiation.

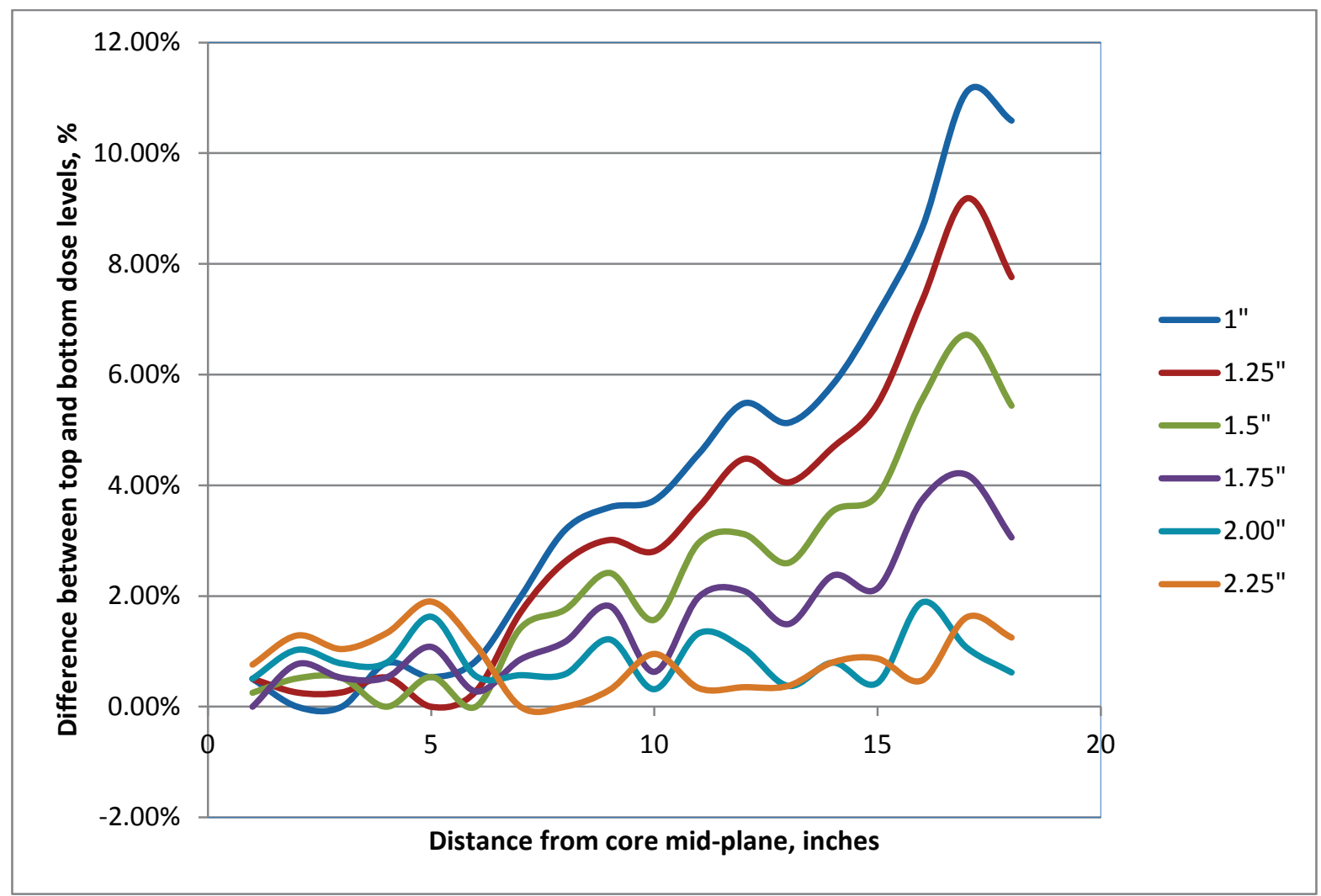

Figure 6. Calculated difference between top and bottom stack dose levels.

It should be noted that the specimen offset in the lower half of the AGC-1 capsule is significantly different from the offset calculated for the AGC-3 capsule [14,15]. AGC-1 used an older ATR flux profile which did not accurately reflect the current flux profiles within the South Flux Trap. As a consequence, the off-set was approximately 3.75 inches (15 piggyback specimen heights) leading to slightly larger differences between the upper and lower matched specimen pairs than expected [14]. This will need to be addressed in detail in the AGC-1 PIE report to be issued after PIE is complete.

\subsubsection{Determining the Physical Positions of Irradiation Creep Specimens in the Stacks}

Once the specimen position offset was established for the bottom half of the specimens, the number of total creep specimens for each type of graphite were determined. To increase the number of creep specimens in the AGC-3 test series capsule, most of the 0.25 -in.-tall NBG- 25 graphite spacers between creep specimens were eliminated. This decision to eliminate all spacers except those containing flux wires increased the total number of specimens by over 20 in the entire capsule. This allowed more specimens per graphite type to be irradiated within the AGC-3 capsule.

A further decision was made to increase the creep specimen number population for the newer graphite types since there is little to no irradiation data on these types. Specifically, more specimens of NBG-18 
and PCEA were chosen to be irradiated than the IG-110, and 2114 graphite grades. NBG-18 and PCEA were determined to have 16 specimens per applied stress level for a total of 48 specimens within AGC-3. Graphite types IG-110 and Mersen 2114 only had 14 specimens per applied stress level for a total of 42 specimens within AGC-3. NBG-17 had the fewest at 12 specimens per load for a total of 36 total creep specimens with AGC-3. The total number of creep specimens irradiated per graphite grade are shown in Table 2.

Table 2. Total number of irradiated creep specimens in AGC-3 capsule.

\begin{tabular}{|c|c|}
\hline Graphite & Total creep specimen \\
\hline PCEA & 48 \\
\hline NBG-18 & 48 \\
\hline IG-110 & 42 \\
\hline 2114 & 42 \\
\hline NBG-17 & 36 \\
\hline
\end{tabular}

A number of other factors were considered before the stacking order for each stack could be finalized. First, there are three load levels applied to the specimens in the loaded upper parts of the stacks (loads of $13.8,17$, and $21 \mathrm{MPa}$ ). There are six outer stacks in the capsule allowing the specimens in two of the stacks to be loaded at $13.8 \mathrm{MPa}$, with specimens loaded at 17 and $21 \mathrm{MPa}$, respectively, in the other two pairs of stacks. Since there are two stacks at similar applied stress levels, the specimen loading order can be shifted between the two stacks allowing the same type of graphite loaded at the specimen stress levels to be exposed over a broader neutron dose range (Figure 7). Assuming that both stacks will have the same applied stress, receive similar dose levels per position, and have a constant temperature allows for this shifting of the specimens and more uniform, smoother dose profile for each graphite type.

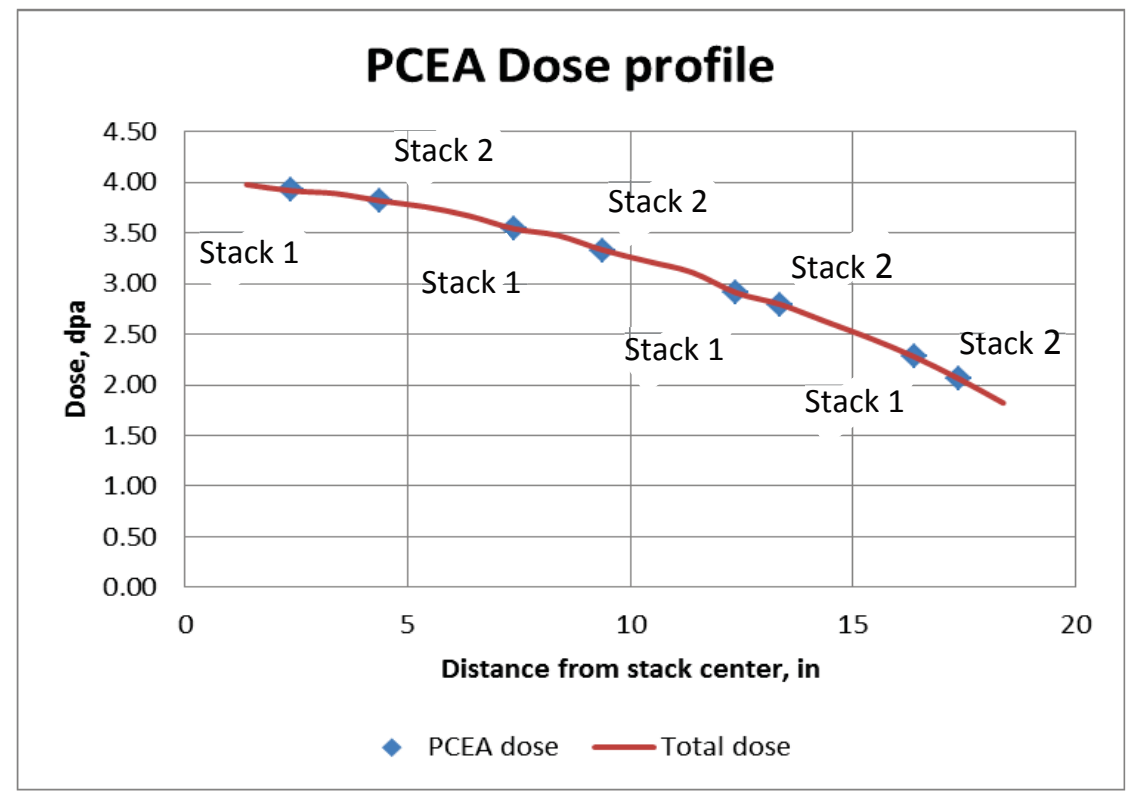

Figure 7. Typical AGC-3 dose profile for creep graphite specimens utilizing similar applied stress in matched stacks.

The second consideration is the grain orientation of the specimens. A decision was made to have approximately $75 \%$ of the specimens be orientated in the "with grain" direction and $25 \%$ of the specimens 
be "against grain". However, in the case of the vibration-molded graphite types (NBG-18 and NBG-17), there are actually two with grain directions and one against grain direction as a consequence of the fabrication process [16]. As such, it was logical to split the with grain and against grain specimens evenly rather than the $75 / 25$ ratio established for the other specimens.

Once these considerations were accounted for, the dose level profiles were determined for each graphite type and within each stack. The estimated creep specimen dose profiles for each graphite type at each stress level are illustrated in the charts for Figure 8.
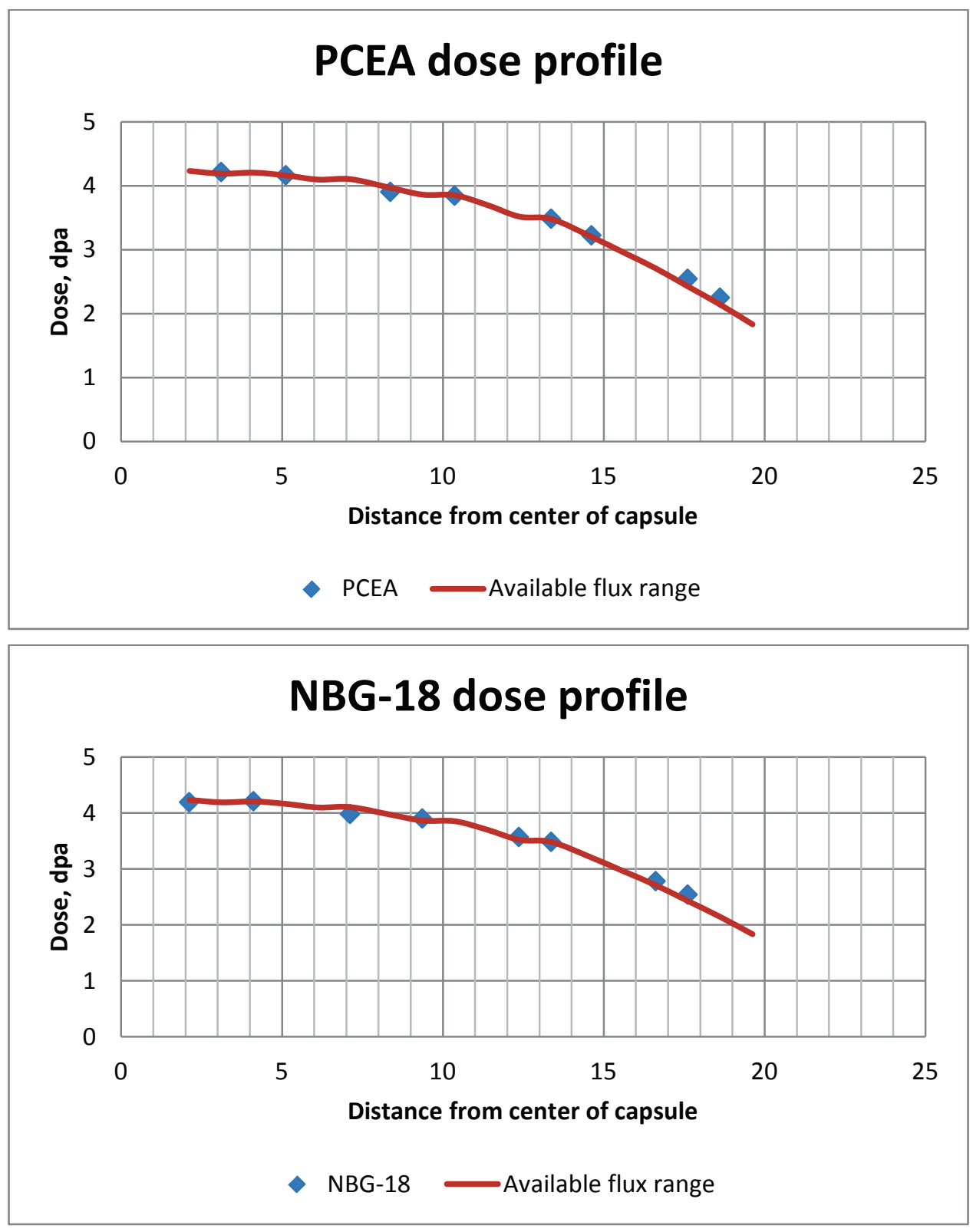

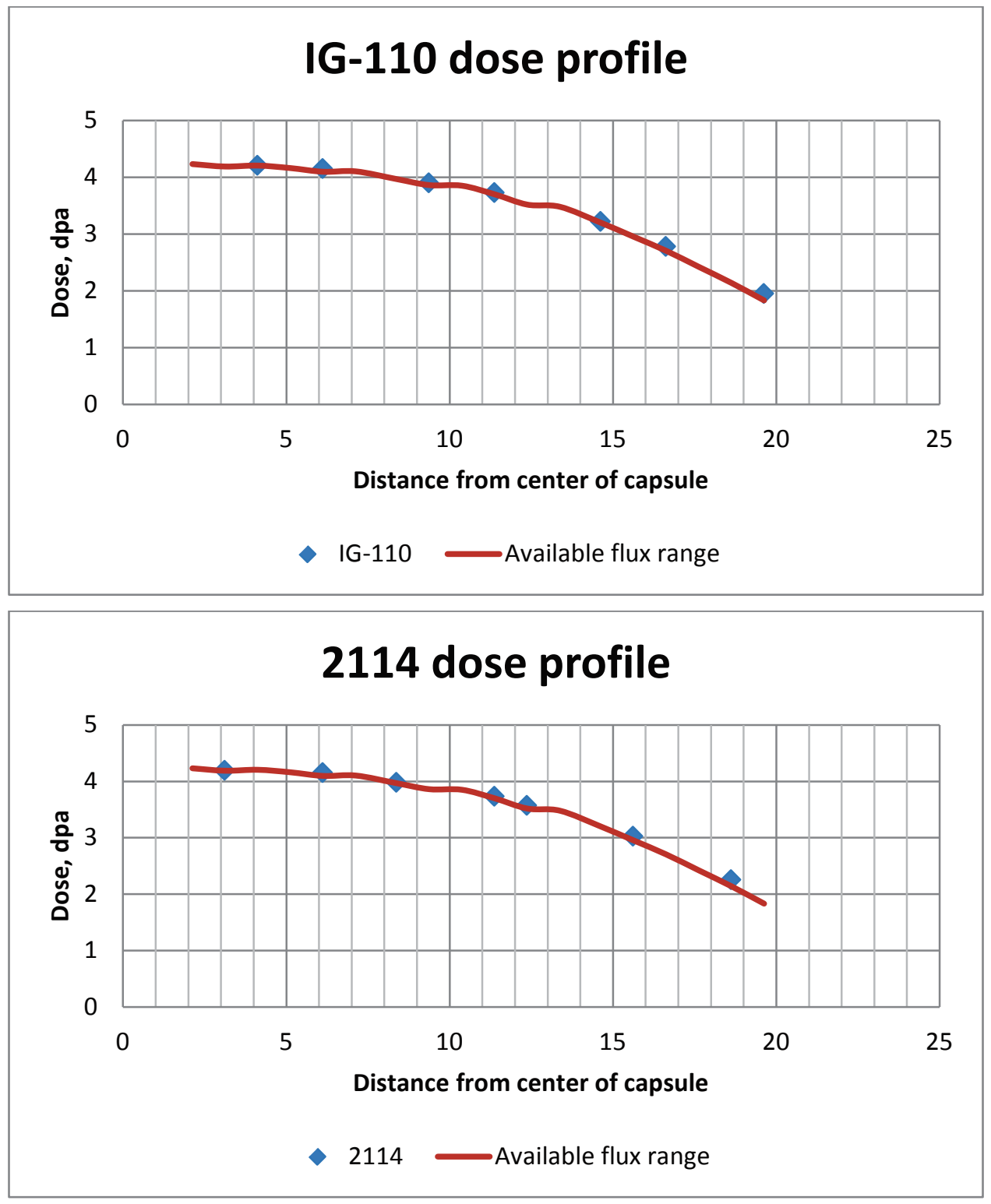


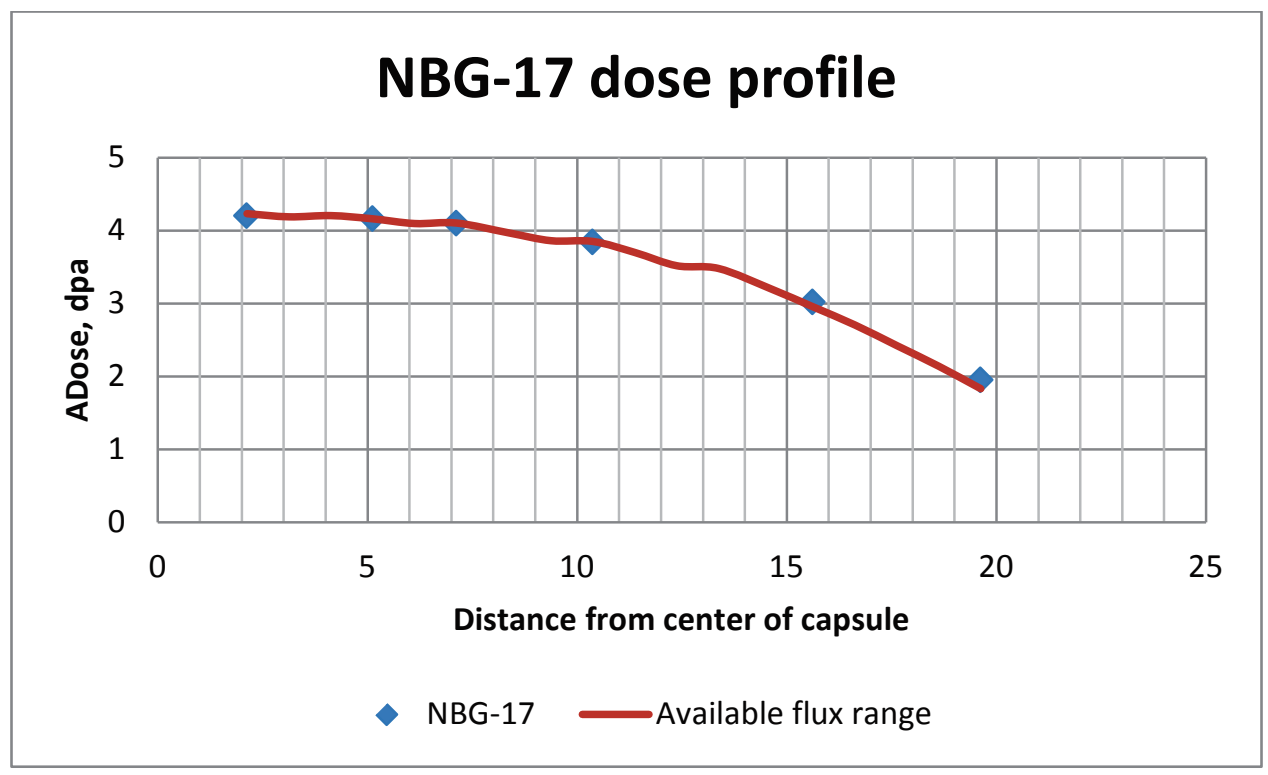

Figure 8. Estimated creep specimen dose profiles for each major graphite type

The final loading configuration for the outer stacks was established once a smooth dose profile was achieved for each graphite type (Table 3). The lower stack offset, the flux wire spacers, creep specimens, and specimen symmetry above and below the capsule mid-plane were mapped for each graphite specimen.

Table 3. Final loading configuration for AGC-3 creep specimens in outer stacks.

\begin{tabular}{|l|l|l|l|}
\hline \multicolumn{5}{|c|}{ Stack 1 } \\
\hline \multicolumn{1}{|c|}{ Orientation } & ID No. & \multicolumn{1}{|c|}{ Graphite } & Height \\
\hline $\begin{array}{l}\text { Stack End Cap } \\
\text { NBG-25 }\end{array}$ & & & \\
\hline AP & AP3001 & NBG-17 & 19.625 \\
\hline & & FLUX Wire & 18.875 \\
\hline DW & DW3001 & PCEA & 18.250 \\
\hline BW & BW3001 & NBG-18 & 17.250 \\
\hline EW & EW3001 & IG-110 & 16.250 \\
\hline TW & TW3001 & 2114 & 15.250 \\
\hline DW & DW3004 & PCEA & 14.250 \\
\hline & & FLUX Wire & 13.625 \\
\hline BP & BP3002 & NBG-18 & 13.000 \\
\hline TW & TW3004 & 2114 & 12.000 \\
\hline EW & EW3004 & IG-110 & 11.000 \\
\hline DW & DW3103 & PCEA & 10.000 \\
\hline BW & BW3101 & NBG-18 & 9.000 \\
\hline TW & TW3705 & 2114 & 8.000 \\
\hline & & FLUX Wire & 7.375 \\
\hline
\end{tabular}




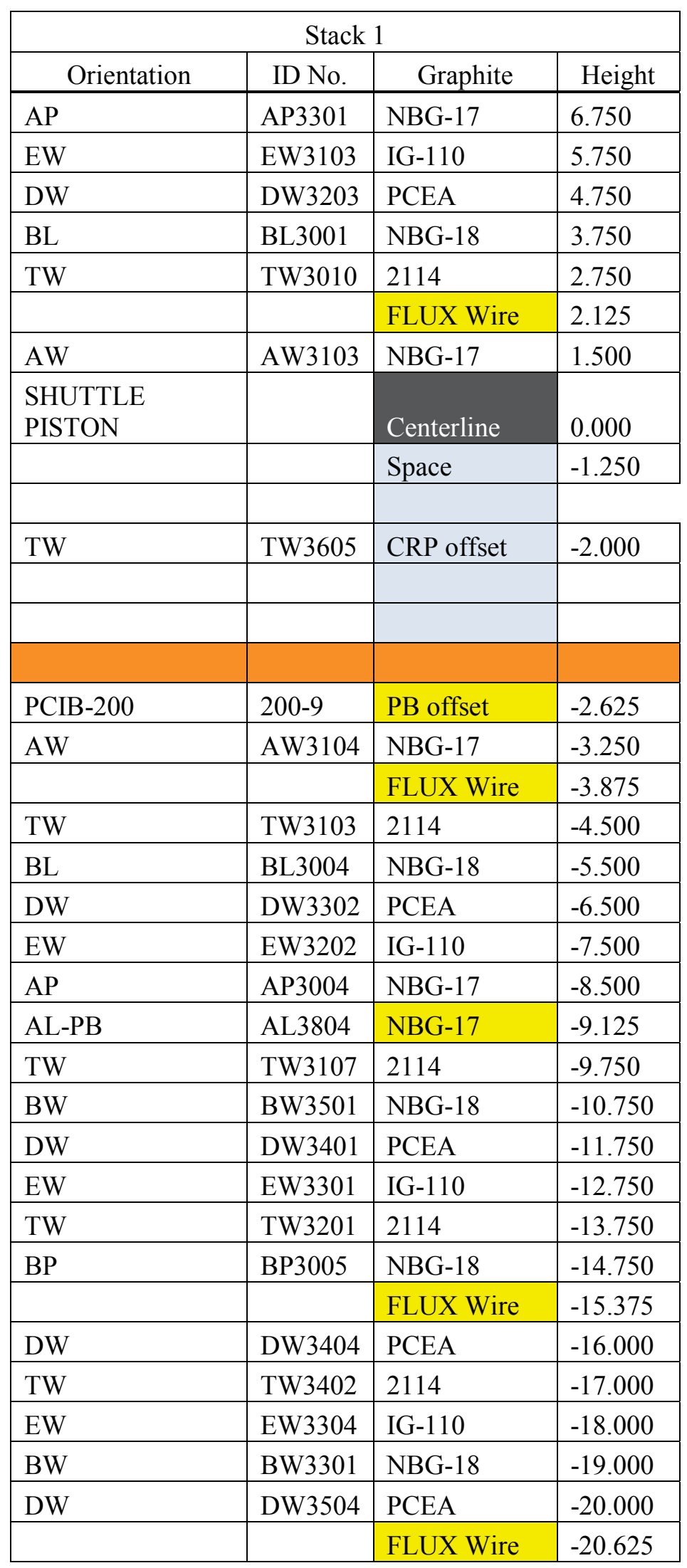




\begin{tabular}{|l|c|l|c|}
\hline \multicolumn{4}{|c|}{ Stack 1 } \\
\hline \multicolumn{1}{|c|}{ Orientation } & ID No. & \multicolumn{1}{c|}{ Graphite } & Height \\
\hline AP & AP3202 & NBG-17 & -21.250 \\
\hline & & & \\
\hline TW & TW3608 & CRP offset & -22.250 \\
\hline & & & \\
\hline & & & \\
\hline PCIB-325 & $325-12$ & PB offset & -22.875 \\
\hline PCIB-200 & $200-12$ & PB offset & -23.125 \\
\hline AL-PB & AL3805 & PB offset & -23.375 \\
\hline
\end{tabular}

\begin{tabular}{|l|l|l|l|}
\hline \multicolumn{5}{|c|}{ Stack 2 } \\
\hline \multicolumn{1}{|c|}{ Orientation } & ID No. & \multicolumn{1}{c|}{ Graphite } & Height \\
\hline $\begin{array}{l}\text { Stack End Cap } \\
\text { NBG-25 }\end{array}$ & & & \\
\hline AP & AP3002 & NBG-17 & 19.625 \\
\hline EA & EA-SP1 & IG-110 & 18.875 \\
\hline DW & DW3002 & PCEA & 18.250 \\
\hline BW & BW3002 & NBG-18 & 17.250 \\
\hline EW & EW3802 & IG-110 & 16.250 \\
\hline TW & TW3002 & 2114 & 15.250 \\
\hline DW & DW3101 & PCEA & 14.250 \\
\hline & & FLUX Wire & 13.625 \\
\hline BP & BP3003 & NBG-18 & 13.000 \\
\hline TW & TW3005 & 2114 & 12.000 \\
\hline EW & EW3101 & IG-110 & 11.000 \\
\hline DW & DW3201 & PCEA & 10.000 \\
\hline BW & BW3402 & NBG-18 & 9.000 \\
\hline TW & TW3008 & 2114 & 8.000 \\
\hline EA & EA-SP2 & IG-110 & 7.375 \\
\hline AP & AP3302 & NBG-17 & 6.750 \\
\hline EW & EW3104 & IG-110 & 5.750 \\
\hline DW & DW3204 & PCEA & 4.750 \\
\hline BL & BL3002 & NB-18 & 3.750 \\
\hline TW & TW3101 & 2114 & 2.750 \\
\hline & & FLUX Wire & 2.125 \\
\hline AW & AW3201 & NBG-17 & 1.500 \\
\hline SHUTTLE & & & \\
PISTON & & Centerline & 0.000 \\
\hline
\end{tabular}




\begin{tabular}{|c|c|c|c|}
\hline \multicolumn{4}{|c|}{ Stack 2} \\
\hline Orientation & ID No. & Graphite & Height \\
\hline & & Space & -1.250 \\
\hline TW & TW3606 & CRP offset & -2.000 \\
\hline & & & \\
\hline PCIB-200 & $200-10$ & PB offset & -2.625 \\
\hline AW & AW3202 & NBG-17 & -3.250 \\
\hline EA-PB & EA3609 & IG-110 & -3.875 \\
\hline TW & TW3105 & 2114 & -4.500 \\
\hline $\mathrm{BL}$ & BL3101 & NBG-18 & -5.500 \\
\hline DW & DW3303 & PCEA & -6.500 \\
\hline EW & EW3203 & IG-110 & -7.500 \\
\hline $\mathrm{AP}$ & AP3101 & NBG-17 & -8.500 \\
\hline EA-PB & EA3610 & IG-110 & -9.125 \\
\hline TW & TW3108 & 2114 & -9.750 \\
\hline $\mathrm{BW}$ & BW3202 & NBG-18 & -10.750 \\
\hline DW & DW3402 & PCEA & -11.750 \\
\hline EW & EW3302 & IG-110 & -12.750 \\
\hline TW & TW3202 & 2114 & -13.750 \\
\hline $\mathrm{BP}$ & BP3101 & NBG-18 & -14.750 \\
\hline & & FLUX Wire & -15.375 \\
\hline DW & DW3501 & PCEA & -16.000 \\
\hline TW & TW3403 & 2114 & -17.000 \\
\hline EW & EW3401 & IG-110 & -18.000 \\
\hline BW & BW3503 & NBG-18 & -19.000 \\
\hline DW & DW3601 & PCEA & -20.000 \\
\hline EA-PB & EA3611 & IG-110 & -20.625 \\
\hline AP & AP3203 & NBG-17 & -21.250 \\
\hline TW & TW3609 & CRP offset & -22.250 \\
\hline PCIB-325 & $325-13$ & PB offset & -22.875 \\
\hline PCIB-200 & $200-13$ & PB offset & -23.125 \\
\hline AL-PB & AL3806 & $P B$ offset & -23.375 \\
\hline
\end{tabular}




\begin{tabular}{|c|c|c|c|}
\hline \multicolumn{4}{|c|}{ Stack 3} \\
\hline Orientation & ID No. & Graphite & Height \\
\hline $\begin{array}{l}\text { Stack End Cap } \\
\text { NBG-25 }\end{array}$ & & & 19.625 \\
\hline $\mathrm{AP}$ & AP3003 & NBG-17 & 19.500 \\
\hline $\mathrm{EW}$ & EW-SP1 & IG-110 & 18.875 \\
\hline DW & DW3003 & PCEA & 18.250 \\
\hline $\mathrm{BW}$ & BW3401 & NBG-18 & 17.250 \\
\hline EW & EW3003 & IG-110 & 16.250 \\
\hline TW & TW3003 & 2114 & 15.250 \\
\hline DW & DW3102 & PCEA & 14.250 \\
\hline & & FLUX Wire & 13.625 \\
\hline BP & BP3004 & NBG-18 & 13.000 \\
\hline $\mathrm{TW}$ & TW3006 & 2114 & 12.000 \\
\hline $\mathrm{EW}$ & EW3801 & IG-110 & 11.000 \\
\hline DW & DW3202 & PCEA & 10.000 \\
\hline $\mathrm{BW}$ & BW3103 & NBG-18 & 9.000 \\
\hline TW & TW3009 & 2114 & 8.000 \\
\hline EW & EW-SP2 & IG-110 & 7.375 \\
\hline $\mathrm{AP}$ & AP3303 & NBG-17 & 6.750 \\
\hline EW & EW3201 & IG-110 & 5.750 \\
\hline DW & DW3301 & PCEA & 4.750 \\
\hline $\mathrm{BL}$ & BL3003 & NB-18 & 3.750 \\
\hline TW & TW3102 & 2114 & 2.750 \\
\hline & & FLUX Wire & 2.125 \\
\hline $\mathrm{AW}$ & AW3203 & NBG-17 & 1.500 \\
\hline $\begin{array}{l}\text { SHUTTLE } \\
\text { PISTON }\end{array}$ & & Centerline & 0.000 \\
\hline & & Space & -1.250 \\
\hline TW & TW3607 & CRP offset & -2.000 \\
\hline & & & \\
\hline & & & \\
\hline PCIB-200 & $200-11$ & PB offset & -2.625 \\
\hline $\mathrm{AW}$ & AW3204 & NBG-17 & -3.250 \\
\hline EW-PB & EW4607 & IG-110 & -3.875 \\
\hline TW & TW3106 & 2114 & -4.500 \\
\hline $\mathrm{BL}$ & BL3102 & NBG-18 & -5.500 \\
\hline
\end{tabular}




\begin{tabular}{|c|c|c|c|}
\hline \multicolumn{4}{|c|}{ Stack 3} \\
\hline Orientation & ID No. & Graphite & Height \\
\hline DW & DW3304 & PCEA & -6.500 \\
\hline EW & EW3204 & IG-110 & -7.500 \\
\hline $\mathrm{AP}$ & AP3102 & NBG-17 & -8.500 \\
\hline EW-PB & EW4608 & IG-110 & -9.125 \\
\hline TW & TW3109 & 2114 & -9.750 \\
\hline $\mathrm{BW}$ & BW3502 & NBG-18 & -10.750 \\
\hline DW & DW3403 & PCEA & -11.750 \\
\hline EW & EW3303 & IG-110 & -12.750 \\
\hline TW & TW3704 & 2114 & -13.750 \\
\hline \multirow[t]{2}{*}{$\mathrm{BP}$} & BP3102 & NBG-18 & -14.750 \\
\hline & & FLUX Wire & -15.375 \\
\hline DW & DW3502 & PCEA & -16.000 \\
\hline $\mathrm{TW}$ & TW3404 & 2114 & -17.000 \\
\hline EW & EW3402 & IG-110 & -18.000 \\
\hline $\mathrm{BW}$ & BW3303 & NBG-18 & -19.000 \\
\hline DW & DW3602 & PCEA & -20.000 \\
\hline EW-PB & EW4609 & IG-110 & -20.625 \\
\hline $\mathrm{AP}$ & AP3204 & NBG-17 & -21.250 \\
\hline TW & TW3610 & CRP offset & -22.250 \\
\hline PCIB-325 & $325-14$ & $P B$ offset & -22.875 \\
\hline PCIB-200 & $200-14$ & PB offset & -23.125 \\
\hline AL-PB & AL3807 & PB offset & -23.375 \\
\hline
\end{tabular}

\begin{tabular}{|l|l|l|l|}
\hline \multicolumn{5}{|c|}{ Stack 4 } \\
\hline \multicolumn{1}{|c|}{ Orientation } & ID No. & \multicolumn{1}{c|}{ Graphite } & Height \\
\hline $\begin{array}{l}\text { Stack End Cap } \\
\text { NBG-25 }\end{array}$ & & & \\
\hline EW & EW3601 & IG-110 & 19.625 \\
\hline & & FLUX Wire & 18.875 \\
\hline TW & TW3703 & 2114 & 18.250 \\
\hline DA & DA3001 & PCEA & 17.250 \\
\hline BP & BP3103 & NBG-18 & 16.250 \\
\hline AL & AL3202 & NBG-17 & 15.250 \\
\hline EW & EW3604 & IG-110 & 14.250 \\
\hline
\end{tabular}




\begin{tabular}{|c|c|c|c|}
\hline \multicolumn{4}{|c|}{ Stack 4} \\
\hline \multirow[t]{2}{*}{ Orientation } & ID No. & Graphite & Height \\
\hline & & FLUX Wire & 13.625 \\
\hline DW & DW3801 & PCEA & 13.000 \\
\hline $\mathrm{BL}$ & BL3103 & NBG-18 & 12.000 \\
\hline TW & TW3405 & 2114 & 11.000 \\
\hline AW & AW3001 & NBG-17 & 10.000 \\
\hline EA & EA3001 & IG-110 & 9.000 \\
\hline \multirow[t]{2}{*}{$\mathrm{DA}$} & DA3203 & PCEA & 8.000 \\
\hline & & FLUX Wire & 7.375 \\
\hline $\mathrm{BP}$ & BP3202 & NBG-18 & 6.750 \\
\hline TW & TW3408 & 2114 & 5.750 \\
\hline $\mathrm{AP}$ & AP3304 & NBG-17 & 4.750 \\
\hline EA & EA3004 & IG-110 & 3.750 \\
\hline \multirow[t]{2}{*}{ DW } & DW3603 & PCEA & 2.750 \\
\hline & & FLUX Wire & 2.125 \\
\hline $\mathrm{BP}$ & BP3301 & NBG-18 & 1.500 \\
\hline \multirow[t]{2}{*}{$\begin{array}{l}\text { SHUTTLE } \\
\text { PISTON } \\
\end{array}$} & & Centerline & 0.000 \\
\hline & & Space & -1.250 \\
\hline AW-PB & AW3810 & PB offset & -1.625 \\
\hline EW-PB & EW4601 & PB offset & -1.875 \\
\hline TW-PB & TW3821 & PB offset & -2.125 \\
\hline PCIB-325 & $325-9$ & PB offset & -2.375 \\
\hline PCIB-PB & P3-07 & PB offset & -2.625 \\
\hline \multirow[t]{2}{*}{$\mathrm{BP}$} & BP3304 & NBG-18 & -3.250 \\
\hline & & FLUX Wire & -3.875 \\
\hline DW & DW3702 & PCEA & -4.500 \\
\hline EA & EA3103 & IG-110 & -5.500 \\
\hline AP & AP3403 & NBG-17 & -6.500 \\
\hline TW & TW3501 & 2114 & -7.500 \\
\hline $\mathrm{BP}$ & BP3603 & NBG-18 & -8.500 \\
\hline TW-PB & TW3904 & 2114 & -9.125 \\
\hline DA & DA3302 & PCEA & -9.750 \\
\hline EA & EA3202 & IG-110 & -10.750 \\
\hline AW & AW3004 & NBG-17 & -11.750 \\
\hline TW & TW3504 & 2114 & -12.750 \\
\hline $\mathrm{BL}$ & BL3201 & NBG-18 & -13.750 \\
\hline
\end{tabular}




\begin{tabular}{|l|l|l|l|}
\hline \multicolumn{5}{|c|}{ Stack 4 } \\
\hline \multicolumn{1}{|c|}{ Orientation } & ID No. & \multicolumn{1}{c|}{ Graphite } & Height \\
\hline DW & DW3803 & PCEA & -14.750 \\
\hline \multicolumn{5}{|c|}{} & FLUX Wire & -15.375 \\
\hline EW & EW3403 & IG-110 & -16.000 \\
\hline AL & AL3301 & NBG-17 & -17.000 \\
\hline BP & BP3801 & NBG-18 & -18.000 \\
\hline DA & DA3403 & PCEA & -19.000 \\
\hline TW & TW3507 & 2114 & -20.000 \\
\hline \multicolumn{5}{|c|}{} & FLUX Wire & -20.625 \\
\hline EW & EW3502 & IG-110 & -21.250 \\
\hline BL-PB & BL3601 & PB offset & -21.875 \\
\hline AL-PB & AL3801 & $P B$ offset & -22.125 \\
\hline EW-PB & EW4509 & PB offset & -22.375 \\
\hline AW-PB & AW3901 & PB offset & -22.625 \\
\hline DW-PB & DW4511 & $P B$ offset & -22.875 \\
\hline DA-PB & DA3510 & $P B$ offset & -23.125 \\
\hline TW-PB & TW3827 & $P B$ offset & -23.375 \\
\hline
\end{tabular}

\begin{tabular}{|l|l|l|l|}
\hline \multicolumn{5}{|c|}{ Stack 5 } \\
\hline \multicolumn{1}{|c|}{ Orientation } & ID No. & \multicolumn{1}{c|}{ Graphite } & Height \\
\hline $\begin{array}{l}\text { Stack End Cap } \\
\text { NBG-25 }\end{array}$ & & & \\
\hline EW & EW3602 & IG-110 & 19.625 \\
\hline DA-PB & DA-SP1 & PCEA & 19.500 \\
\hline TW & TW3702 & 2114 & 18.875 \\
\hline DA & DA3003 & PCEA & 17.250 \\
\hline BP & BP3104 & NBG-18 & 16.250 \\
\hline AL & AL3203 & NBG-17 & 15.250 \\
\hline EW & EW3701 & IG-110 & 14.250 \\
\hline & & FLUX Wire & 13.625 \\
\hline DW & DW3804 & PCEA & 13.000 \\
\hline BL & BL3104 & NBG-18 & 12.000 \\
\hline TW & TW3406 & 2114 & 11.000 \\
\hline AW & AW3002 & NBG-17 & 10.000 \\
\hline EA & EA3002 & IG-110 & 9.000 \\
\hline DA & DA3204 & PCEA & 8.000 \\
\hline DA-PB & DA-SP2 & PCEA & 7.375 \\
\hline BP & BP3204 & NBG-18 & 6.750 \\
\hline
\end{tabular}




\begin{tabular}{|c|c|c|c|}
\hline \multicolumn{4}{|c|}{ Stack 5} \\
\hline Orientation & ID No. & Graphite & Height \\
\hline TW & TW3409 & 2114 & 5.750 \\
\hline $\mathrm{AP}$ & AP3401 & NBG-17 & 4.750 \\
\hline EA & EA3101 & IG-110 & 3.750 \\
\hline \multirow[t]{2}{*}{ DW } & DW3604 & PCEA & 2.750 \\
\hline & & FLUX Wire & 2.125 \\
\hline $\mathrm{BP}$ & BP3302 & NBG-18 & 1.500 \\
\hline \multirow[t]{2}{*}{$\begin{array}{l}\text { SHUTTLE } \\
\text { PISTON } \\
\end{array}$} & & Centerline & 0.000 \\
\hline & & Space & -1.250 \\
\hline AW-PB & AW3811 & PB offset & -1.625 \\
\hline EW-PB & EW4602 & PB offset & -1.875 \\
\hline TW-PB & TW3822 & PB offset & -2.125 \\
\hline PCIB-325 & $325-10$ & PB offset & -2.375 \\
\hline PCIB-PB & P3-08 & PB offset & -2.625 \\
\hline $\mathrm{BP}$ & BP3601 & NBG-18 & -3.250 \\
\hline DA-PB & DA3603 & PCEA & -3.875 \\
\hline DW & DW3703 & PCEA & -4.500 \\
\hline EA & EA3104 & IG-110 & -5.500 \\
\hline $\mathrm{AP}$ & AP3404 & NBG-17 & -6.500 \\
\hline TW & TW3502 & 2114 & -7.500 \\
\hline $\mathrm{BP}$ & BP3604 & NBG-18 & -8.500 \\
\hline DA-PB & DA3604 & PCEA & -9.125 \\
\hline DA & DA3303 & PCEA & -9.750 \\
\hline EA & EA3203 & IG-110 & -10.750 \\
\hline AW & AW3101 & NBG-17 & -11.750 \\
\hline TW & TW3505 & 2114 & -12.750 \\
\hline $\mathrm{BL}$ & BL3202 & NBG-18 & -13.750 \\
\hline \multirow[t]{2}{*}{ DW } & DW3901 & PCEA & -14.750 \\
\hline & & FLUX Wire & -15.375 \\
\hline EW & EW3704 & IG-110 & -16.000 \\
\hline $\mathrm{AL}$ & AL3101 & NBG-17 & -17.000 \\
\hline $\mathrm{BP}$ & BP3702 & NBG-18 & -18.000 \\
\hline DA & DA3404 & PCEA & -19.000 \\
\hline TW & TW3508 & 2114 & -20.000 \\
\hline DA-PB & DA3605 & PCEA & -20.625 \\
\hline EW & EW3503 & IG-110 & -21.250 \\
\hline
\end{tabular}




\begin{tabular}{|l|l|l|c|}
\hline \multicolumn{5}{|c|}{ Stack 5 } \\
\hline \multicolumn{1}{|c|}{ Orientation } & ID No. & \multicolumn{1}{c|}{ Graphite } & Height \\
\hline BL-PB & BL3602 & PB offset & -21.875 \\
\hline AL-PB & AL3802 & PB offset & -22.125 \\
\hline EW-PB & EW4510 & PB offset & -22.375 \\
\hline AW-PB & AW3902 & PB offset & -22.625 \\
\hline DW-PB & DW4512 & PB offset & -22.875 \\
\hline DA-PB & DA3511 & PB offset & -23.125 \\
\hline TW-PB & TW3828 & PB offset & -23.375 \\
\hline
\end{tabular}

\begin{tabular}{|c|c|c|c|}
\hline \multicolumn{4}{|c|}{ Stack 6} \\
\hline Orientation & ID No. & Graphite & Height \\
\hline $\begin{array}{l}\text { Stack End Cap } \\
\text { NBG-25 }\end{array}$ & & & 19.625 \\
\hline EW & EW3603 & IG-110 & 19.500 \\
\hline DA-PB & DA-SP3 & PCEA & 18.875 \\
\hline TW & TW3602 & 2114 & 18.250 \\
\hline $\mathrm{DA}$ & DA3004 & PCEA & 17.250 \\
\hline $\mathrm{BP}$ & BP3201 & NBG-18 & 16.250 \\
\hline $\mathrm{AL}$ & AL3204 & NBG-17 & 15.250 \\
\hline \multirow[t]{2}{*}{ EW } & EW3702 & IG-110 & 14.250 \\
\hline & & FLUX Wire & 13.625 \\
\hline DW & DW3902 & PCEA & 13.000 \\
\hline $\mathrm{BL}$ & BL3204 & NBG-18 & 12.000 \\
\hline TW & TW3407 & 2114 & 11.000 \\
\hline AW & AW3003 & NBG-17 & 10.000 \\
\hline EA & EA3003 & IG-110 & 9.000 \\
\hline DA & DA3301 & PCEA & 8.000 \\
\hline DA-PB & DA-SP4 & PCEA & 7.375 \\
\hline $\mathrm{BP}$ & BP3205 & NBG-18 & 6.750 \\
\hline TW & TW3410 & 2114 & 5.750 \\
\hline $\mathrm{AP}$ & AP3402 & NBG-17 & 4.750 \\
\hline EA & EA3102 & IG-110 & 3.750 \\
\hline \multirow[t]{2}{*}{ DW } & DW3701 & PCEA & 2.750 \\
\hline & & FLUX Wire & 2.125 \\
\hline $\mathrm{BP}$ & BP3303 & NBG-18 & 1.500 \\
\hline \multirow[t]{2}{*}{$\begin{array}{l}\text { SHUTTLE } \\
\text { PISTON }\end{array}$} & & Centerline & 0.000 \\
\hline & & Space & -1.375 \\
\hline
\end{tabular}




\begin{tabular}{|c|c|c|c|}
\hline \multicolumn{4}{|c|}{ Stack 6} \\
\hline Orientation & ID No. & Graphite & Height \\
\hline AW-PB & AW3812 & PB offset & -1.625 \\
\hline EW-PB & EW4603 & PB offset & -1.875 \\
\hline TW-PB & TW3823 & PB offset & -2.125 \\
\hline PCIB-325 & $325-11$ & PB offset & -2.375 \\
\hline PCIB-PB & P3-09 & PB offset & -2.625 \\
\hline $\mathrm{BP}$ & BP3602 & NBG-18 & -3.250 \\
\hline DA-PB & DA3608 & PCEA & -3.875 \\
\hline DW & DW3704 & PCEA & -4.500 \\
\hline EA & EA3201 & IG-110 & -5.500 \\
\hline $\mathrm{AP}$ & AP3103 & NBG-17 & -6.500 \\
\hline TW & TW3503 & 2114 & -7.500 \\
\hline $\mathrm{BP}$ & BP3605 & NBG-18 & -8.500 \\
\hline DA-PB & DA3607 & PCEA & -9.125 \\
\hline $\mathrm{DA}$ & DA3401 & PCEA & -9.750 \\
\hline EA & EA3204 & IG-110 & -10.750 \\
\hline AW & AW3102 & NBG-17 & -11.750 \\
\hline TW & TW3506 & 2114 & -12.750 \\
\hline $\mathrm{BL}$ & BL3203 & NBG-18 & -13.750 \\
\hline \multirow[t]{2}{*}{ DW } & DW3903 & PCEA & -14.750 \\
\hline & & FLUX Wire & -15.375 \\
\hline EW & EW3501 & IG-110 & -16.000 \\
\hline $\mathrm{AL}$ & AL3103 & NBG-17 & -17.000 \\
\hline $\mathrm{BP}$ & BP3703 & NBG-18 & -18.000 \\
\hline DA & DA3104 & PCEA & -19.000 \\
\hline TW & TW3509 & 2114 & -20.000 \\
\hline DA-PB & DA3606 & PCEA & -20.625 \\
\hline EW & EW3504 & IG-110 & -21.250 \\
\hline BL-PB & BL3603 & $P B$ offset & -21.875 \\
\hline AL-PB & AL3803 & $P B$ offset & -22.125 \\
\hline EW-PB & EW4511 & $P B$ offset & -22.375 \\
\hline AW-PB & AW3903 & $P B$ offset & -22.625 \\
\hline DW-PB & DW4601 & $P B$ offset & -22.875 \\
\hline DA-PB & DA3512 & $P B$ offset & -23.125 \\
\hline TW-PB & TW3829 & $P B$ offset & -23.375 \\
\hline
\end{tabular}




\subsubsection{Determining the Physical Positions of Piggy-Back Specimens in the Central Stack}

Similar to the AGC-1 and AGC-2 designs, the AGC-3 piggyback specimens are contained within the central stack and did not have a designed applied stress imposed. Similar to the AGC-2 design, AGC-3 piggyback specimens did not have a central hole machined in the center of the specimens. This allowed them to be tested for high temperature thermal diffusivity.

\section{PREIRRADIATION MATERIAL PROPERTY MEASUREMENTS}

The objective of the AGC-3 experiment is to determine the material property changes induced in nuclear grade graphite during exposure to a high-temperature neutron environment. The approach is to perform extensive preirradiation characterization testing on each specimen before exposing the graphite specimens to various neutron doses. After irradiation, the same characterization tests will be performed on each irradiated specimen to ascertain the quantitative changes to the material properties of the graphite.

A brief summary of the material testing is in Table 4. These measurements include metrology (before and after heating to $1000^{\circ} \mathrm{C}$ in the dilatometer and laser flash apparatus [LFA]) and non-destructive characterization of the physical properties. The properties measured were bulk density by mensuration, electrical resistivity, elastic constants, including flexural dynamic Young's modulus (fundamental frequency method), and sonic elastic constants, including Young's modulus, shear modulus, and Poisson's ratio; ambient temperature thermal conductivity, and thermal expansion $\left(\mathrm{RT}-1000^{\circ} \mathrm{C}\right)$.

Table 4. Graphite measurement and test equipment.

\begin{tabular}{|c|c|c|c|c|}
\hline Measurement & Standard & Instrumentation & Calibration Method & Result \\
\hline $\begin{array}{l}\text { Physical } \\
\text { Dimensions and } \\
\text { Mass }\end{array}$ & $\begin{array}{l}\text { ASTM C559-90 } \\
\text { (Reapproved } \\
\text { 2010) }\end{array}$ & $\begin{array}{l}\text { Mitutoyo Micrometer } \\
\text { 121-155 } \\
\text { INL ID: } 725884 \\
\text { INL ID: } 727312 \\
\text { Mitutoyo Caliper CD-6-in. } \\
\text { CSX } \\
\text { INL ID: } 725813 \\
\text { INL ID: } 726607 \\
\text { INL ID: } 727194 \\
\text { Sartorius Scale ME235P } \\
\text { INL ID: } 412642 \\
\text { INL ID: } 415907\end{array}$ & $\begin{array}{l}\text { INL Standards and } \\
\text { Calibration Laboratory }\end{array}$ & Bulk density \\
\hline $\begin{array}{l}\text { Fundamental } \\
\text { Frequency }\end{array}$ & $\begin{array}{l}\text { ASTM C747-93 } \\
\text { (Reapproved } \\
2010 \text { ) } \\
\text { ASTM C1259-08 }\end{array}$ & $\begin{array}{l}\text { J. W. Lemmens Grindosonic } \\
\text { INL ID: } 412850\end{array}$ & $\begin{array}{l}\text { No calibration required } \\
\text { per instrument } \\
\text { manufacturer }\end{array}$ & Elastic modulus \\
\hline Sonic Velocity & ASTM C769-09 & $\begin{array}{l}\text { Olympus NDT Sq. Wave } \\
\text { Pulser/Receiver 5077PR } \\
\text { INL ID: } 728024 \\
\text { National Instruments } \\
\text { Digitizer USB } 5133 \\
\text { INL ID: } 726725 \\
\text { INL ID: } 415868\end{array}$ & $\begin{array}{l}\text { INL Standards and } \\
\text { Calibration Laboratory }\end{array}$ & $\begin{array}{l}\text { Young's } \\
\text { modulus, Shear } \\
\text { modulus, } \\
\text { Poisson ratio }\end{array}$ \\
\hline
\end{tabular}




\begin{tabular}{|l|l|l|l|l|}
\hline \multicolumn{1}{|c|}{ Measurement } & \multicolumn{1}{|c|}{ Standard } & \multicolumn{1}{c|}{ Instrumentation } & \multicolumn{1}{c|}{ Calibration Method } & \multicolumn{1}{c|}{ Result } \\
\hline $\begin{array}{l}\text { 4-point Electrical } \\
\text { Resistivity }\end{array}$ & $\begin{array}{l}\text { ASTM C611-98 } \\
\text { (Reapproved } \\
\text { 2010) }\end{array}$ & $\begin{array}{l}\text { Kiethly 6220 Precision } \\
\text { Current Source } \\
\text { INL ID: 725865 } \\
\text { INL ID: 727290 } \\
\text { Kiethly 2182A Nano } \\
\text { Voltmeter } \\
\text { INL ID: 725866 } \\
\text { INL ID: 727289 }\end{array}$ & $\begin{array}{l}\text { INL Standards and } \\
\text { Calibration Laboratory }\end{array}$ & $\begin{array}{l}\text { Electrical } \\
\text { resistivity }\end{array}$ \\
\hline $\begin{array}{l}\text { Laser Flash } \\
\text { Diffusivity }\end{array}$ & ASTM E1461-07 & $\begin{array}{l}\text { Netzsch LFA 457 2 ea. } \\
\text { INL ID: 412855 } \\
\text { INL ID: 412864 }\end{array}$ & $\begin{array}{l}\text { Calibration by user per } \\
\text { manufacturer's } \\
\text { instructions }\end{array}$ & $\begin{array}{l}\text { Thermal } \\
\text { diffusivity }\end{array}$ \\
\hline $\begin{array}{l}\text { Push Rod } \\
\text { Dilatometry }\end{array}$ & ASTM E228-06 & $\begin{array}{l}\text { Netzsch DIL 402 C 2 ea. } \\
\text { INL ID: 412860 } \\
\text { INL ID: 412861 }\end{array}$ & $\begin{array}{l}\text { Calibration by user per } \\
\text { manufacturer's } \\
\text { instructions }\end{array}$ & $\begin{array}{l}\text { Coefficient of } \\
\text { thermal } \\
\text { expansion }\end{array}$ \\
\hline $\begin{array}{l}\text { Environmental } \\
\text { Monitoring }\end{array}$ & ALL & $\begin{array}{l}\text { Visala Pressure, Humidity } \\
\text { and Temperature PTU301 } \\
\text { INL ID: 726912 } \\
\text { INL ID: 727884 } \\
\text { INL ID: 727502 }\end{array}$ & $\begin{array}{l}\text { INL Standards and } \\
\text { Calibration Laboratory }\end{array}$ & $\begin{array}{l}\text { Laboratory } \\
\text { environmental } \\
\text { conditions }\end{array}$ \\
\hline
\end{tabular}

The measurements listed in Table 4 are segregated into individual stations that consist of the instrumentation necessary, a computer for automated data acquisition, and a bar code reader. The bar code of the individual specimen container is read and the file for that specimen is automatically opened for data input prior to each measurement. Associated with each measurement type is a unique laboratory notebook maintained to INL procedure Management Control Procedure (MCP)-2875 Rev 11, "Proper Use and Maintenance of Laboratory Notebooks," and Plan (PLN)-2690 Rev 9, "VHTR Technology Development Office Quality Assurance Program Plan," Paragraph 3.3. Accepted data will be stored in the NGNP Data Management and Analysis System (NDMAS), a satellite file location for NGNP. Data in a standardized Excel file format will be transmitted to NDMAS using Form 435.78 Rev 2, "VHTR Technology Development Office Information Input Sheet," following PLN-3319 Rev 2, "Records Management Plan for the VHTR Technology Development Office Program."

In addition to laboratory notebooks, the specific measuring instruments are networked to a server computer where the measurement data is automatically stored. This has been implemented in the INL Carbon Characterization Laboratory where custom LabVIEW software was written to facilitate automated data acquisition. This software is comprised of five main programs: Manufacturers Data, Physical and Dimensional Measurements, Electrical Resistivity Measurements, Sonic Resonance (Fundamental Frequency) Measurements, and Sonic Velocity Measurements. These five programs acquire data from instrumentation or user input and record the results in an Excel spreadsheet located on a server computer. In the case of thermal expansion and thermal diffusivity measurements, two other LabVIEW programs have also been written to parse vendor software acquired data into Excel spreadsheets. MCP-3058, "VHTR TDO Software Quality Assurance," and FRM-959-1 Rev 1; "VHTR Software Management Plan for Quality Level 1 or Safety Software used to implement MCP-3058 are currently used to govern the development, accuracy, and configuration control of this software.

Following is the sequence in which the measurements are made:

1. Wash and dry - All specimens. 
2. Mass and dimensional measurements - All specimens.

3. Thermal diffusivity - Piggy Back specimens.

4. Elastic modulus by sonic resonance-Creep specimens.

5. Electrical resistivity - Creep specimens.

6. Elastic modulus by measurement of sonic velocity - Creep specimens.

7. Wash and dry to remove couplant - Creep specimens.

8. Coefficient of thermal expansion - Creep specimens.

9. Post characterization of mass and dimensional measurements - All specimens.

\subsection{General Provisions}

The AGC-3 specimens have been characterized per PLN-3858 "AGC-3 Graphite Specimen Preirradiation Characterization Plan.” This plan describes the thermal, physical, and mechanical measurement techniques and methods that were used to characterize the different graphite types being tested in the AGC-3 experiment and is intended to meet the requirements of MCP-1380, "Research and Development Test Control," and NQA-1-2008; 1a-2009, "Quality Assurance Requirements for Nuclear Facility Applications"; requirement 11, Test Control. Described within the plan are the instruments, fixtures, and methods used for preirradiation material property measurements of bulk density, thermal diffusivity, coefficient of thermal expansion, elastic modulus, and electrical resistivity.

All work was performed in accordance with Laboratory-wide Procedure (LWP) LWP-21220, "Work Management." All records designated in implementing documents as quality assurance records were controlled in accordance with the records plan PLN-3319, "Records Management Plan for the VHTR Technology Development Office Program."

The data resulting from the preirradiation characterization are plotted in Appendix A and listed in Appendix C. Statistical evaluation has been performed using an inner quartile range analysis to identify levels of uncertainty and outliers in the data. The measured properties and characteristics of different graphite types will be compared along with the effect of grain orientation.

\subsection{Specimen Description and Preparation}

Major grades of the nuclear-grade graphite types to be tested in AGC-3 are NBG-17, NBG-18, PCEA, 2114, and IG-110. Minor grades of graphite include NBG-25, PCIB, and HOPG. All major grades have been characterized fully per PLN-3858 and the minor grades have only had dimensional, density, and thermal diffusivity measurements performed on them. The two primary specimen types in the AGC experiments are creep specimens and piggyback specimens. Creep specimens from major grade graphite types are shown in INL Drawing 601501, Rev. 4, and will be subjected to a mechanical load during irradiation to induce irradiation creep within the specimens. Piggyback specimens from both major and minor grade graphite types are also shown in INL Drawing 601501, Rev. 4. They are not subjected to a mechanical load and are subjected only to neutron irradiation at high operating temperatures to assess the effects of a reactor environment on the specific graphite grade.

All specimens are $12.5 \mathrm{~mm}$ in diameter, with the creep specimens being $25.4 \mathrm{~mm}$ long and the piggyback specimens being $6 \mathrm{~mm}$ long. Details of how specimens were cut from the graphite blocks are also contained in INL Drawing 601501, Rev. 4, "ATR Advanced Graphite Capsule (AGC-3) Experiment Graphite Specimen Cut-Out Diagrams."

Immediately after being machined, each specimen is placed in an individual container that is bar coded with a unique identification number per INL Drawing 601501, Rev. 4. Each graphite specimen is 
then laser engraved with that same unique identification number around the circumference at one end. Prior to any material property measurement, each specific specimen is identified by its unique identification number and the data is recorded/stored under this identification number. After the specimens have been laser engraved, they are ultrasonically cleaned as follows:

1. Handle the specimens only while wearing cotton or powder-free nitrile gloves.

2. Remove all dust and debris using an aerosol pressurized dust-off product.

3. Ultrasonically clean specimens for 20 minutes in deionized water.

4. Rinse specimens in ethyl alcohol to help displace water.

5. Allow to air dry.

6. Place specimens in a laboratory oven at $130^{\circ} \mathrm{C}$ for 2 hours.

7. Allow specimens to cool in a desiccator and retain there in storage until resistivity or bulk density measurements are taken.

It should be noted that irradiated specimens are not washed again prior to characterization measurements. However, for measurements of density and resistivity, Steps 6 and 7 above are followed. That is to say, the irradiated specimens are dried in a laboratory oven at $130^{\circ} \mathrm{C}$ for 2 hours and allowed to cool in a desiccator where they are retained until resistivity and/or bulk density measurements are performed.

\subsection{Personnel and Training}

Personnel who perform measurements identified in this plan are qualified in accordance with MCP-3052, "VHTR TDO Personnel Qualification and Certification." Their ability to adequately perform measurements described in this plan is demonstrated by instrument manufacturers' training and certification and/or performance of an instrument/measurement operational validation. Personnel qualifications are reviewed by the Graphite R\&D lead and documented in laboratory notebooks.

\subsection{Variations, Exceptions and Discrepancies}

There are several variations, exceptions, and discrepancies that may occur. The first is a known departure from the applicable American Society for Testing and Materials (ASTM) standard. These departures are typically related to geometrical constraints. All currently known departures or exceptions taken to the ASTM standard are described in detail in Section 3 of this plan. Any departure not captured in this plan will be recorded in laboratory notebooks associated with the measurement. In most cases, the effects of the exception or departure from the ASTM method on the measured value are not well understood. When possible, sensitivity studies will be performed and documented in laboratory notebooks to understand the impact of these exceptions and departures.

It is likely that the ASTM standards and/or test methods will be revised and improved during the more than 10-year-long AGC experiment cycle. Each revision or development will be evaluated for how it could impact future measurements and their consistency with measurements made under previous revisions or techniques. A programmatic determination will be made whether to continue with the current version of the ASTM/method or use the updated version. This determination will be documented in laboratory notebooks associated with the affected measurement.

While measurements are being made, it is possible that something out of the ordinary may occur. Any unusual event that occurs during a measurement will be documented in the laboratory notebook associated with that specific measurement and duly noted within the database associated with the data generated for this program. The principle investigator (PI) will be notified of the event and will determine 
what impact it has on the data. The significance of the result will be documented in the laboratory notebook by the PI.

\subsection{Calibration and Functional Validation}

The measurement protocol consists of calibration, functional validation, and data acquisition. Functional validations established for each measurement in collaboration with the instrument manufacturer are performed periodically to ensure that accurate and consistent data is acquired. All validations are performed on traceable standards and documented in retrievable laboratory notebooks associated with each measurement. In the event that an instrument functional validation fails, the reason for the failure is investigated and resolved prior to that measurement being used for further characterization. Upon resolution, a determination is made as to the impact the failure might have had on data taken prior to the failure and back to the last valid measurement. If it is determined the data captured during this interval is suspect, the impacted data will be evaluated for accuracy.

MCP-3066, "VHTR TDO Control of Measuring and Test Equipment," is followed for calibration standards, methods, and frequencies that have been established for each measurement. Where it is not possible to use the INL Standards and Calibration Laboratory, calibration by user procedures are established based on ASTM standards and manufacturers' instructions and performed against international standards. These procedures are documented in laboratory notebooks associated with each measurement.

\section{TEST METHODS}

Before any measurements are made, specimen numbers and basic information about each type of graphite are entered into the manufacturer's data program. Once basic information about the graphite type has been recorded, it is automatically saved to an Excel spreadsheet file and the individual specimen numbers are entered using a bar code reader. Following the initial input of general information, individual material property measurements are made starting with mass and dimensional measurements for determining bulk density.

\subsection{Mass, Dimensions and Bulk Density}

Dimensional change is one of the key parameters affecting the performance of graphite in a neutron environment. Determining volumetric and linear dimension as a function of temperature and radiological dose is necessary to understand critical performance measures such as dimensional change turnaround, irradiation creep, and internal stresses imposed upon graphite components. Dimensional and mass measurements are performed to ASTM Standard C559-90 (Reapproved 2010), which describes in detail the procedure for making dimensional measurements and calculating bulk density.

The accuracy of the dial micrometers used here is stated by the manufacture to be $2 \mu \mathrm{m}$. This is a $0.008 \%$ accuracy on a $25.4 \mathrm{~mm}$ measurement. However, when evaluating the uncertainty of the density determination other factors must be considered, such as the hardness of the material and the force with which the micrometer blade is engaged with the material, specimen temperature variation, technician skill, etc. These and other factors were considered in a propagation of error analysis to arrive at an uncertainty of $0.08 \%$ with the measurement of the diameter being the largest contributor to the error.

\subsection{Electrical Resistivity}

Electrical resistivity is used as a rapid, simple means to determine grain orientation, structure, and crystallinity of graphite. In conjunction with optical microscopy, it can be used to determine the microstructural texture of graphite components without much specimen preparation work. Resistivity is measured following ASTM C 611-98 (Reapproved 2010). The measurement technique is commonly 
referred to as 4-point probe. It consists of passing a known current through the specimen and measuring the voltage across the specimen at known locations.

Based on Ohms Law, the resistance is determined and the resistivity is calculated from:

$$
\rho=R \cdot A / L
$$

where

$$
\begin{aligned}
& \mathrm{R}=\text { the measured resistance } \\
& \mathrm{A}=\text { the cross sectional area } \\
& \mathrm{L}=\text { the length over which the voltage is measured. }
\end{aligned}
$$

Figure 9 shows a test fixture fabricated at INL that allows a specimen to be rotated for multiple measurements of voltage around its periphery.

Uncertainty in the resistivity measurement is mainly comprised of the contact resistance between the specimen and the contacting blades for the voltage measurement. Specimen temperature and the temperature of other bimetal junctions in the voltage measuring leads are also factors. These effects are minimized by passing the current through the specimen in two directions and averaging the measured voltage for each direction. In this way, any thermoelectric or small differences in junction resistances will cancel. A round robin test series reported in ASTM C 611 precision and bias section states a lab-to-lab variability of $2.5 \%$. A round robin test series such as this would take into account the variables discussed above and is considered a good estimate of the measurement uncertainty.

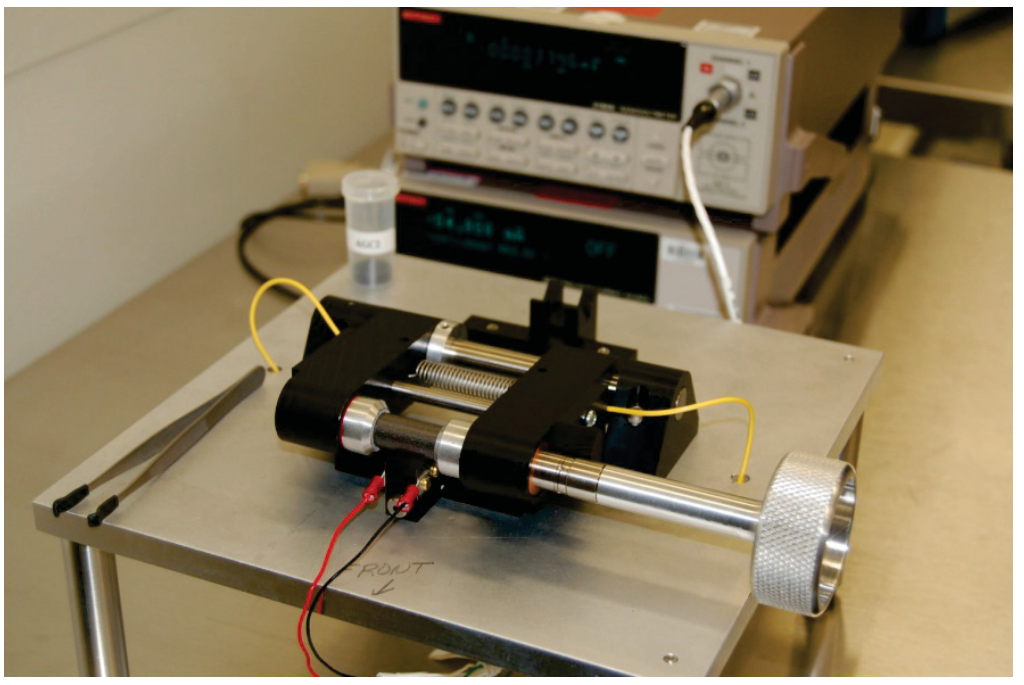

Figure 9. Electrical resistivity measurement station.

\subsection{Approximation of Elastic Modulus from the Measurement of Sonic Velocity}

The mechanical properties of graphite are necessary to determine the structural integrity of graphite components. These properties are vital to determining the viability of the structural strength and integrity of the reactor core. The as-received and irradiated values are needed for whole-core models, which will be used for the graphite design code. This test is carried out in accordance with ASTM C 769-09. In this measurement the transmitting piezoelectric transducer sends a $2.25 \mathrm{MHz}$ sound wave through the specimen. At the opposite end of the specimen, the acoustic wave is received by another piezoelectric 
transducer. The sonic velocity of the specimen is the ratio of specimen length to the signal time lapse between transducers.

An approximate value for Young's modulus, E, can be obtained from:

$$
E=\rho V^{2}
$$

where

$\rho=$ the specimen density

$\mathrm{V}=$ the sonic velocity.

Figure 10 shows the sonic velocity measurement station. In the foreground are the fixtures for clamping the specimen between the transducer and receiver that were specifically designed and fabricated at INL for this application. They have unique features that improve measurement accuracy, precision, and efficiency. Specimens are easily and rapidly loaded into the fixture using the cam-operated clamp. Measurement precision is improved because the spring loaded clamp applies consistent pressure between the transducers and specimen resulting in repeatable couplant thickness.

As specified in Paragraphs 8.1 and 8.5.1 of ASTM C 769-09, a suitable coupling medium should be used and reported with the data. Here, "Shear Gel," manufactured by Sonotech Inc., is used for a shear wave couplant and "Ultragel II," also manufactured by Sonotech Inc., is used for the transverse wave couplant.

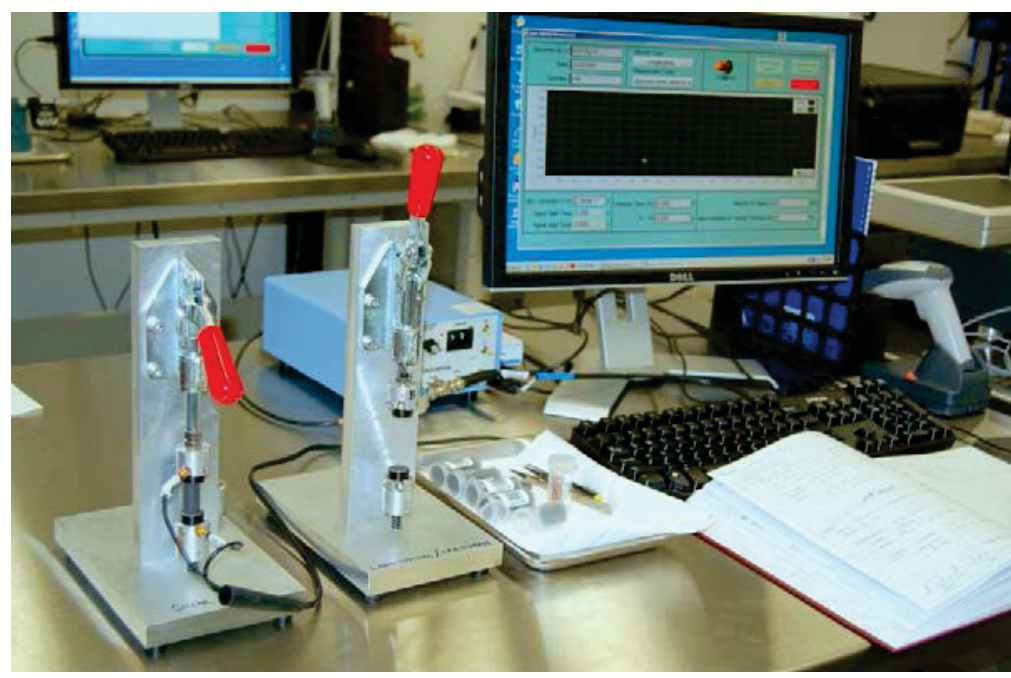

Figure 10. Sonic velocity measurement station.

Figure 11 shows the LabVIEW software user interface display for sonic velocity measurements after scanning the bar code of the specimen to be tested. This screen is used to acquire sonic velocity measurements of a specimen in both the longitudinal and shear directions. Operating much like an oscilloscope, the cursors automatically mark the time between the transmitted wave and the received wave. Also shown in Figure 11 are two examples of the shear wave and transverse wave timing locations for properly coupled specimens. The specimen length divided by this transit time is the sonic velocity.

The uncertainty in determining elastic moduli from the measurement of sonic velocity comes from several sources. First there is the effect of material and geometry related dispersion of the transmitted wave. ASTM C-769 provides guidance on how to minimize this problem by choosing the correct frequency. This technique also assumes linear elastic behavior and graphite is not completely linearly 
elastic. And finally, the operator's judgment on the placement of the timing cursors is somewhat subjective. Clean wave forms to base these judgments on are highly dependent on the quality of the transducer-material coupling. These sources of error are difficult to quantify, and therefore difficult to combine in a propagation of error analysis. However, ASTM C769 describes in some detail a round robin test series between different labs. Using round robin test data to determine a coefficient of variation $(\mathrm{COV})$ is a good means of estimating the measurement uncertainty. With caution, the COV of $3.8 \%$ reported in $\mathrm{C} 769$ is taken here to be representative of the uncertainty of these measurements. When considering a single material and making comparisons between the pre and post-irradiation values, the precision of these measurements is good enough to consider differences greater than $4 \%$ significant. However, one is cautioned to refrain from using the values here as absolute or better than $\pm 10 \%$ accurate.

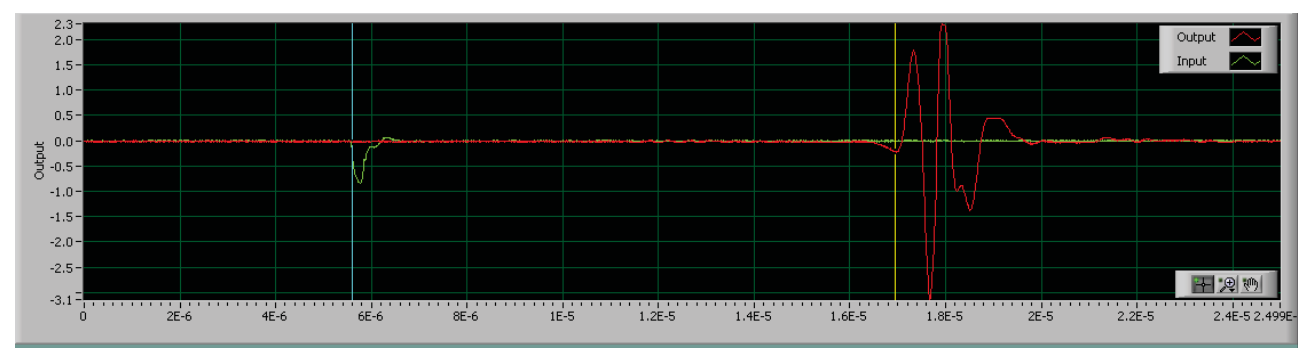

Shear wave timing

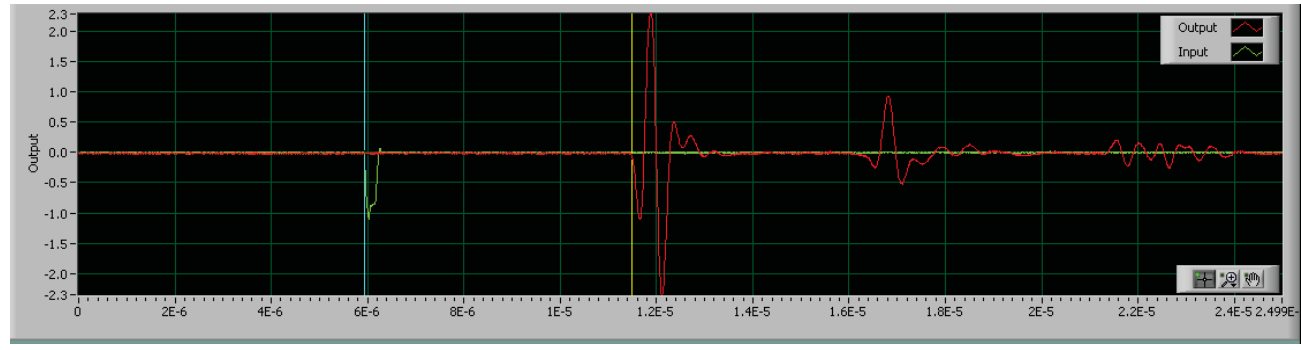

Transverse wave timing

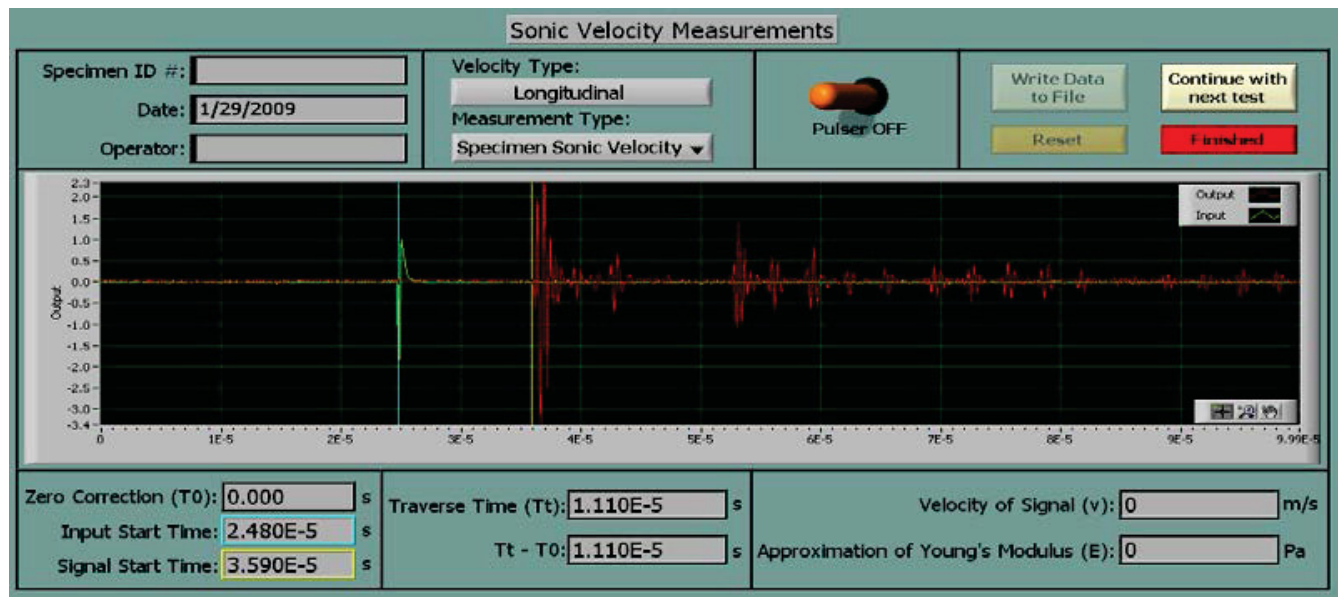

Figure 11. Sonic velocity measurement user interface. 


\subsection{Modulus of Elasticity by Measurement of Fundamental Frequency}

The mechanical properties of graphite are necessary to determine the structural integrity of graphitic components. These properties are vital to determining the viability of the structural strength and integrity of the reactor core. This test method measures the fundamental resonant frequency of test specimens of suitable geometry by exciting them mechanically with a singular elastic strike. Specimen supports, impulse locations, and signal pick-up points are selected to induce and measure specific modes of the transient vibration of the specimen. The transient signals are analyzed, and the fundamental resonant frequency is isolated and measured by the signal analyzer. The measured fundamental resonant frequency, specimen dimensions, and mass are used to calculate the dynamic Young's modulus, shear modulus, and Poisson's ratio per ASTM C747-93 (Reapproved 2010) in combination with apparatus and calculations described in ASTM C1259-08. The fundamental frequency measurement station is shown in Figure 12.

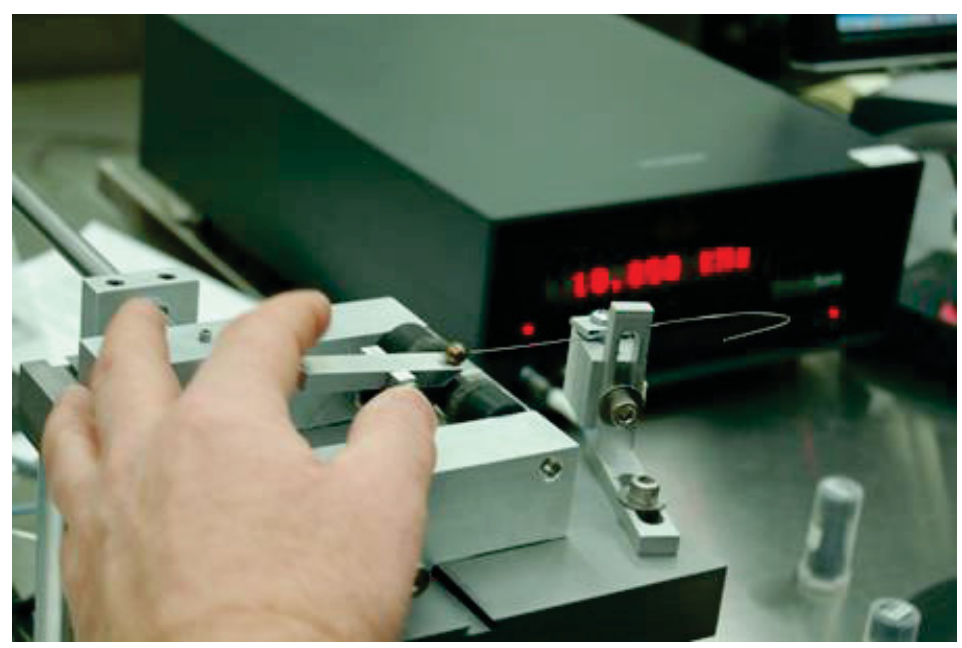

Figure 12. Fundamental frequency measurement station.

After placing a specimen in the test fixture, the user excites it by lightly tapping it with a small mechanical impulse. A consistent impulse is achieved by placing the ball hammer onto a lever that rotates out from under the hammer as it is raised. The specimen is supported in such a way that it vibrates at its natural frequency. A microphone placed underneath one end of the specimen in combination with the Grindosonic electronics measure this frequency, which is recorded and displayed by the computer. The modulus of elasticity is calculated and displayed next to the newly acquired frequency. If the results are satisfactory, the user can press the "Save $1^{\text {st }}$ Frequency" button and go on to the next measurement. Following the recommendations of ASTM C-1259-08, 10 readings of the fundamental frequency are measured before the results of the test are written to the applicable Excel spreadsheet.

ASTM C-1259 describes in detail a round robin test series using ceramic materials along with an analysis of the propagation of errors in the calculation of moduli from the measurement of resonant frequency, geometry, and mass of the specimen. This error analysis shows the major sources of experimental variation are the measurement of the fundamental frequency and the smallest dimension (diameter) of the specimens due to their higher exponent in the modulus calculations. Both the propagation of error analysis and round robin data indicated an uncertainty of less than $2 \%$. However, the creep specimens tested here do not meet the geometry requirements of $\mathrm{C}-1259$. With a length-to-diameter ratio of only 2 , these specimens are, at times, difficult to excite consistently and in a single mode of 
vibration. Once a resonant frequency is determined by an experienced operator for the flexural mode of vibration, it was easily repeated with accuracy within $2 \%$ uncertainty.

\subsection{Thermal Expansion}

Understanding the coefficient of thermal expansion (CTE) for graphite components is critical for determining the dimensional changes that occur as a result of temperature cycles. Localized external stresses can be imposed upon mechanically interlocked graphite core components as the individual pieces suffer differential thermal expansion. Internal stresses can occur within larger graphite components if there is a temperature gradient causing differential expansion within the piece (one side has a higher temperature than the other). Finally, the thermal expansion is highly dependent upon the graphite microstructure, such as orientation/anisotropy, pore size and distribution, and crystallinity. Irradiation damage can significantly alter graphite microstructure and thus CTE values. Determining the extent of the changes as a function of irradiation dose and temperature will be a key parameter for reliable calculation of stress states within graphite components, volumetric changes, and irradiation creep rates.

The CTE measured here follows ASTM E228-06. This test method uses a push-rod-type dilatometer to determine the change in length of a graphite specimen relative to that of the holder as a function of temperature. The temperature is varied over the desired range at a slow constant heating or cooling rate. The linear thermal expansion and mean coefficient of thermal expansion, $\alpha$, are calculated from the recorded data using:

$$
\alpha=\frac{1}{L_{0}} \frac{\Delta L}{\Delta T}
$$

where

$\mathrm{L}_{0}=$ the specimen initial length

$\Delta \mathrm{L}=$ the change in length

$\Delta \mathrm{T}=$ the temperature difference between a specified reference temperature and the temperature at which the change in length was measured.

The Netzsch DIL 402 C commercial system (Figure 13) currently used at INL does not have the capability to cool the specimen below ambient temperature. Therefore, the initial length at $20^{\circ} \mathrm{C}$ is linearly extrapolated from expansion data between $100^{\circ} \mathrm{C}$ and $150^{\circ} \mathrm{C}$ and the mean CTE is calculated from a $20^{\circ} \mathrm{C}$ reference temperature.

The greatest source of experimental error in the dilatometry method described here is the correction made for the expansion of the specimen holder and push rod/LVDT mechanism. This differential between the specimen and the apparatus must be accounted for in order to isolate the specimen expansion only. Studies reported in the precision and bias section of ASTM E228 have indicated that this type of dilatometry can be accurate to $4 \%$ when calibrations are performed carefully. 


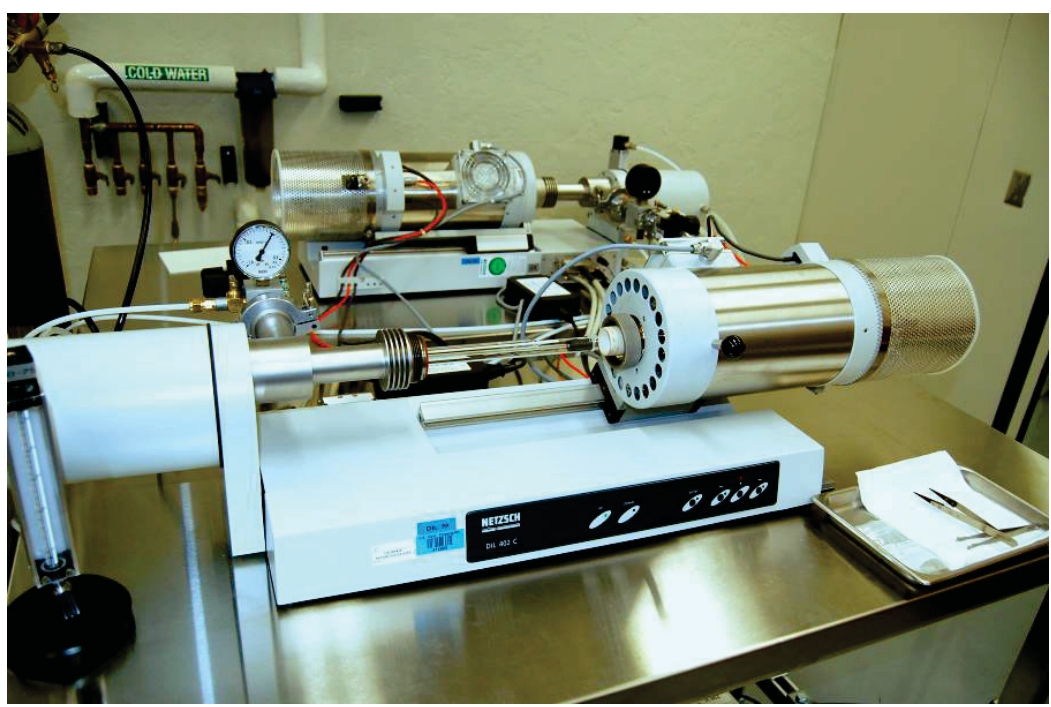

Figure 13. Commercial push rod dilatometer for measurement of CTE.

\subsection{Thermal Diffusivity}

The ability to conduct heat through the graphite core is critical to the passive removal of decay heat. Reduction of the thermal conductivity within graphite can have a significant effect on the passive heat removal rate and thus the peak temperature that the core and, subsequently the fuel particles, will experience during off-normal events. Determining changes to the conductivity as a function of irradiation dose and temperature is important for the HTGR safety analysis. Here, ASTM E1461-07 is followed for the calculation of thermal diffusivity and conductivity. Thermal diffusivity $(\delta)$ is measured and defined as the ratio of thermal conductivity to volumetric heat capacity by:

$$
\delta=\frac{k}{\rho C_{P}}
$$

where

$\mathrm{k}=$ the thermal conductivity

$\rho=$ the density

$C_{P}=$ the specific heat.

The measurement is performed on small, thin, disk-shaped specimens. A pulsed laser is used to subject one surface of the specimen to a high-intensity, short-duration energy pulse. The energy of this pulse is absorbed on the front surface of the specimen and the resulting rise in rear-face temperature is recorded. The thermal diffusivity is calculated from the specimen thickness and the time required for the rear face temperature to reach $50 \%$ of its maximum value. A commercially available LFA, complete with vendor-developed software for instrument control and data acquisition, is used as shown in Figure 14.

Uncertainty in the measurement of thermal diffusivity comes about from specimen heat loss and temperature measurement. Specimen temperature measurement is performed with a calibrated type-S thermocouple in the near vicinity of the specimen. Being a relatively straight forward, the specimen temperature measurement is typically a small contribution to the overall measurement error or uncertainty. 
The main contributor to the measurement uncertainty is heat loss from the specimen. Because this measurement technique depends on the assumption of one-dimensional heat transfer from the flat face receiving the laser pulse to the flat face radiating to the detector, heat loss errors are mainly attributed to radiative heat loss from the circumference of the specimen at temperatures above $300^{\circ} \mathrm{C}$. Typically provided with the instrument software are several correction models to account for this heat loss. As the specimen diameter to thickness ratio decreases, the heat loss increases to the point that the correction models no longer can account for the error. A study was performed to gain a fuller understanding of the limits of the models made available with the NETZSCH LFA and the dependence of the diameter to thickness ratio on measurement error. In this study, the heat loss models were applied to data taken on specimens with various diameter-to-thickness ratios and at specimen temperatures between $25^{\circ} \mathrm{C}$ and $1000^{\circ} \mathrm{C}$. It was determined that the Cowan [17] model along with diameter to thickness ratios greater than or equal to 2 resulted in determination of the diffusivity within ASTM E1461 and the manufactures specified uncertainties of $4 \%$ and $3 \%$, respectively. This was further verified by instrument functional tests performed monthly on a pure iron validation specimen for which the diffusivity was determined to be within $3 \%$ of the Touloukian [18] values between $100^{\circ} \mathrm{C}$ and $700^{\circ} \mathrm{C}$.

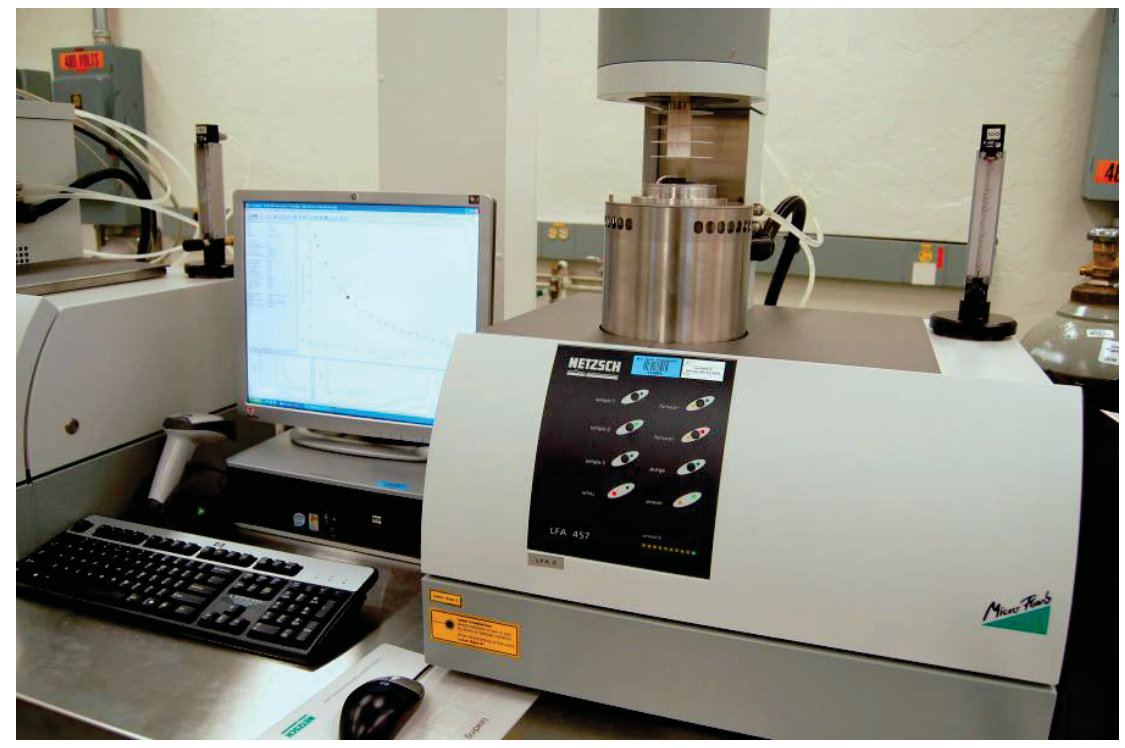

Figure 14. LFA measurement station for determination of thermal diffusivity.

\section{DATA ANALYSIS}

Data gathered for the characterization of AGC-3 specimens are contained in the appendixes of this report. This includes irradiation, sister, and spare specimens as described above. Appendix A contains plots of the individual data points for each specimen. Shown by the dashed lines in each plot are the upper and lower limits of the interquartile range. These limits are established by the lesser of either the least or greatest value in the data or by multiplying the interquartile range (IQR) by 1.5 , and adding or subtracting this value from the third and first quartile. Any data value outside of these limits is a suspected outlier of the established pattern. However, it is important to note that these outlying values are not only subject to measurement variability but also material variability, and therefore cannot necessarily be discarded. These outlying values are examined in the context of the entire data set and will be evaluated further following irradiation. Other statistical parameters are calculated and presented in the tables of Appendix B. The mean, standard deviation, and coefficient of variance are all calculated for the different measurement data sets and graphite types. Upper and lower limits called out in the tables of Appendix B are the IQR limits 
described above. Appendix $\mathrm{C}$ contains all of the raw data including parameters specified by the applicable ASTM (dates, performer ID, room conditions etc.).

There are many ways to combine and compare the data presented here. In doing so, the validity of the data is exercised and scrutinized. First the data sets are considered independently with the statistical analysis described above. Additionally, a limited comparison of the absolute property values is performed between different graphite types and grades. The degree of isotropy is also evaluated for a limited number of grades by calculating the anisotropy ratio.

$$
\text { Anisotropy Ratio }=\frac{\text { Value of the Property in the Against Grain Direction }}{\text { Value of the Property in the With Grain Direction }}
$$

Note that in the case of isostatically molded graphite with grain and against grain indicate specimens taken from orthogonal planes in the billet.

\subsection{Mass, Dimensions and Density Data Analysis}

Plots of the measured mass, dimensions, and density for all AGC-3 specimens are shown in Figures A-1 through A-40 (Appendix A). Beginning with graphite grade 2114, both dimension and mass measurements have relatively low COVs that result in a well behaved calculated values of density. The combined densities of piggyback and creep specimens have a COV of $0.22 \%$, Figure A-1 and A- 6 . Three outliers of the IQR can all be traced back to outliers in the mass measurement.

Variations in the density of NGB-17 were observed that were similar to those observed in AGC-2 preirradiation data. In this case, density trended with location in the billet (Figure A-3 and A-8). Dimensional measurements were consistent and well-behaved for all specimens, but the trend with position in the billet was observed in the mass measurements. To understand this unusual distribution, a study was completed to investigate the density as a function of depth in the NBG-17 billet. Twenty-three specimens $12 \mathrm{~mm} \times 12 \mathrm{~mm} \times 5.5 \mathrm{~mm}$ thick were cut from the top of the billet to a depth of $\sim 125 \mathrm{~mm}$ [16]. Figure 15 shows the density of specimens as a function of depth from the surface. In just $25 \mathrm{~mm}$, the density increases $3 \%$. This low density area or area of inconsistent properties at the outer surface of the billet is often referred to as the rind of the billet.

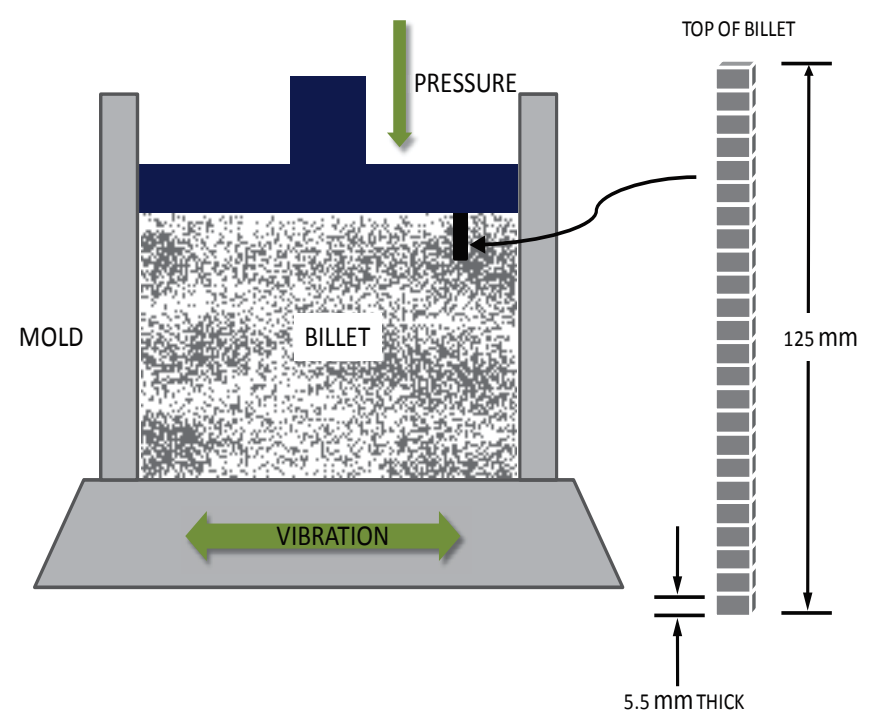

Figure 15. Schematic showing location and dimensional details of NBG-17 billet density investigation. 


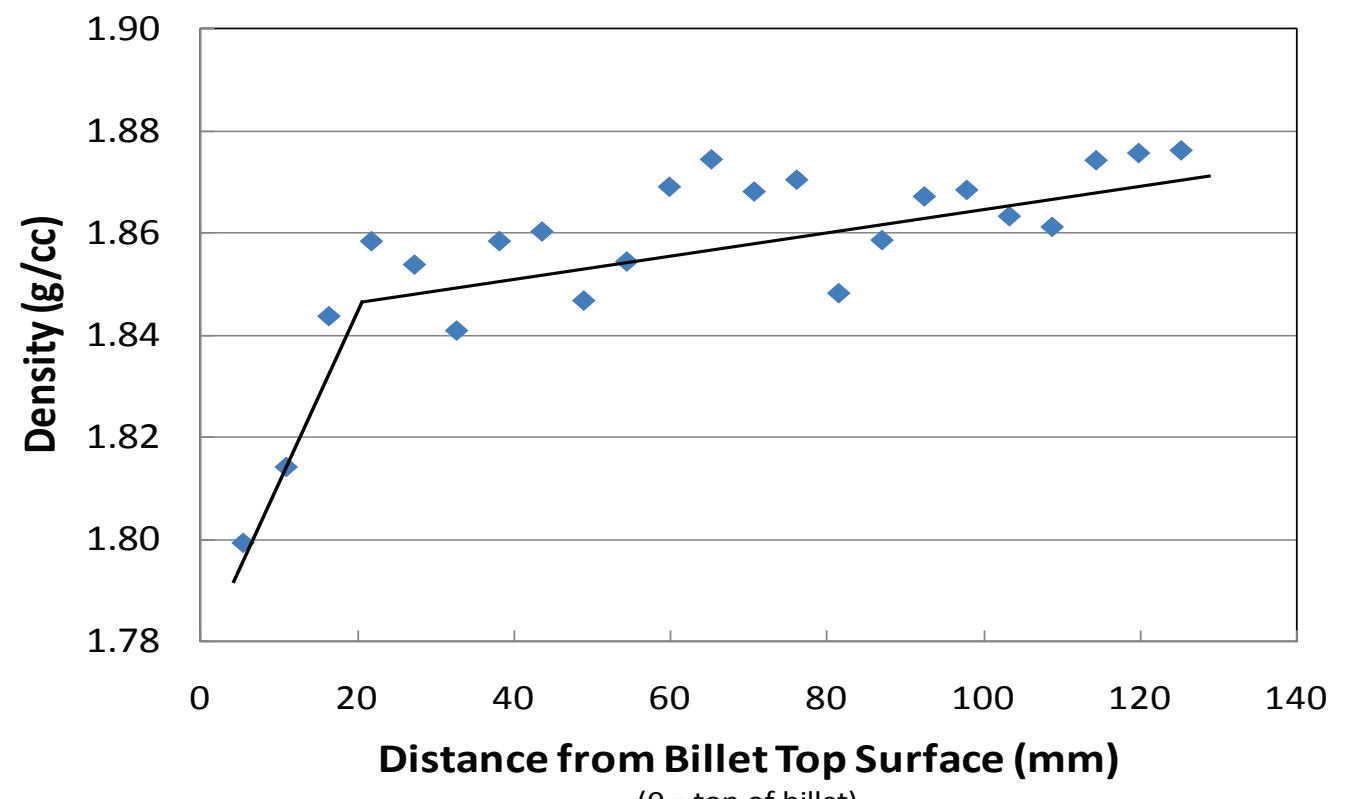

$(0=$ top of billet)

Figure 16. Density of NBG-18 as a function of depth in the billet.

Initially, it appeared unfortunate that specimens were taken from the rind of the billet, but in hind sight this provided a variation in a significant property. With specimens of the same graphite type (i.e., coke, binder, and particle size) other material variables are held constant. This makes it possible to correlate the effect of density on other intrinsic properties like resistivity, elastic modulus, diffusivity, etc. Figure 17 shows an example of this for the elastic modulus measurements of the NBG-17 specimens. As the density of the graphite increases the number of voids and cracks decrease. With the decrease in voids and cracks the graphite becomes less compliant and stiffer, indicated by the increase in Young's modulus.

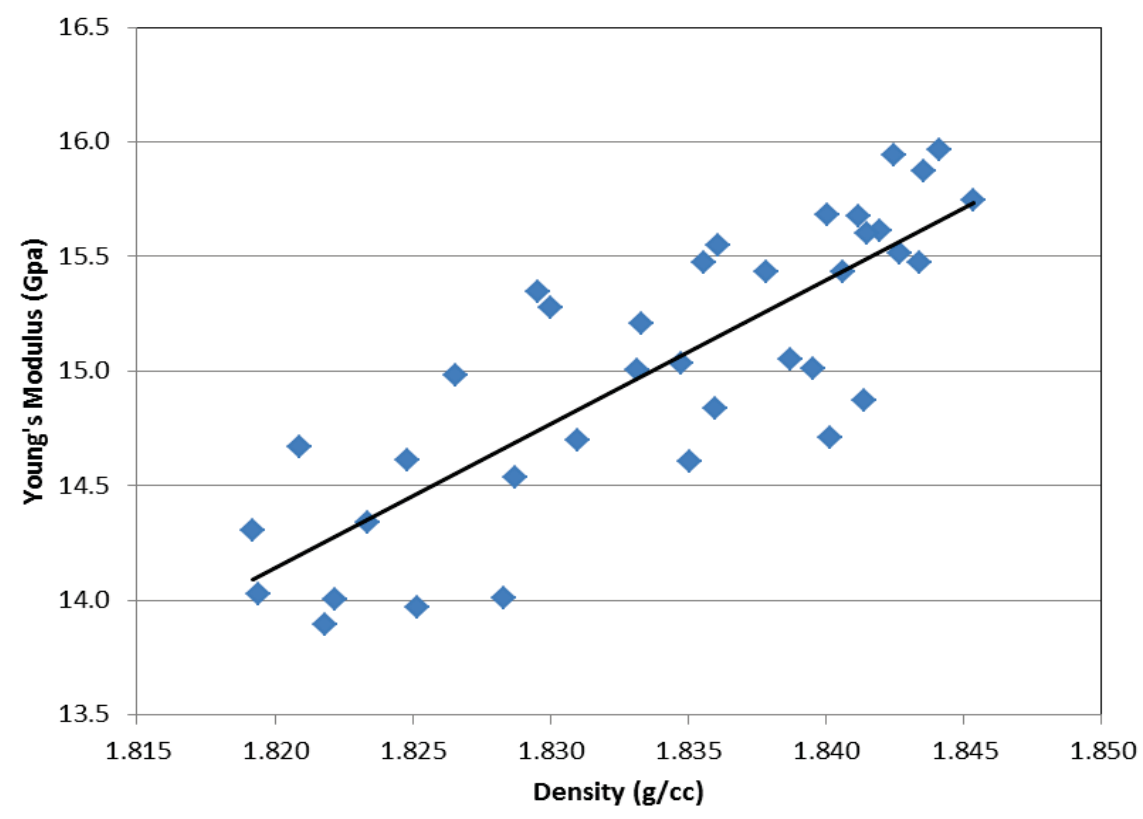

Figure 17. Young's modulus by sonic velocity of NBG-17 as a function of density. 
There are occasions when the determination of bulk density can be used to evaluate outliers or data that is near the IQR limits in either the dimensional or mass measurements. For example, Figures A-15, A-19, and A-20 show several data values of IG-110, NBG-18, and PCEA dimensional measurements that are near or outside the IQR limits. Evaluation of the corresponding density plots (Figures A-5, A-9, and A-10) show all values fall within the quartile limits with relatively low COVs. From this we can conclude that the dimensional outliers are simply specimens that were machined with different dimensions resulting in them being outside or near the limit of the quartile analysis.

Overall the density data of all graphite grades is well behaved and consistent with what was expected. The fact that these density data are so well-behaved indicates any outliers found in the associated dimensional or mass data are simply a result of machining tolerances and are valid.

Additionally, the dimensions and mass were measured following the thermal measurement of the coefficient of thermal expansion and diffusivity. These measurements were performed up to $1000^{\circ} \mathrm{C}$ and concerns existed that the specimens maintained some residual stress due to the machining operations and that this stress would relax during the thermal cycle to $1000^{\circ} \mathrm{C}$. Figures A-81 through A-109 contain plots of a comparison between the pre- and post-thermal treatment dimensions and mass. The comparison plots of specimen length show a slight trend of shrinkage that is on the order of $0.02 \%$. For a $25-\mathrm{mm}$ specimen, the accuracy of the dial micrometers used is $0.01 \%$. The uncertainty in the measurement is not only made up of the dial micrometer accuracy but also other variables such as room temperature, micrometer clutch wear, micrometer blade design, and velocity of blade approach relative to the specimen hardness.

Although there is a definite trend to the length measurements decreasing after the thermal measurements, it is difficult to draw concrete conclusions considering that the length change is probably on the order of the measurement uncertainty. Furthermore, the annealing of residual stress would tend to increase the size of the specimen, not decrease it. However, oxidation could decrease the size of the specimen during the thermal measurements if the furnace is not purged of all air and indeed the post mass measurements trend to lower values by $\sim 0.03 \%$ on average indicating that a very small amount of oxidation was occurring. There are several specimens of graphite type IG-110 (specimen numbers EA3501, EA3502, and EA3503) that displayed mass loss in excess of 3\% (Figures A-107). This suspected oxidation is corroborated in the relatively large reduction of these specimens' length and diameter. These specimens are not used in the irradiation experiment.

\subsection{Electrical Resistivity}

Plots of electrical resistivity are shown in Figures A-66 through A-70 for graphite grades of PCEA, NBG-18, IG-110, and NBG-17. All of the resistivity measurements were performed on the creep specimens only. The COV for all grades is in line with the measurement uncertainty of $2.5 \%$ reported in a round robin test series of ASTM C 611.

An example of a simple comparison between graphite grades and grain orientation is shown in Figure 18. Here the resistivity values of several primary grades of graphite are plotted for both grain orientations and the anisotropy ratio is displayed above the bars. It is interesting to note that all current grades of graphite have good resistivity isotropy. This plot also shows that there is a $25 \%$ difference in resistivity between PCEA and NBG-17. 


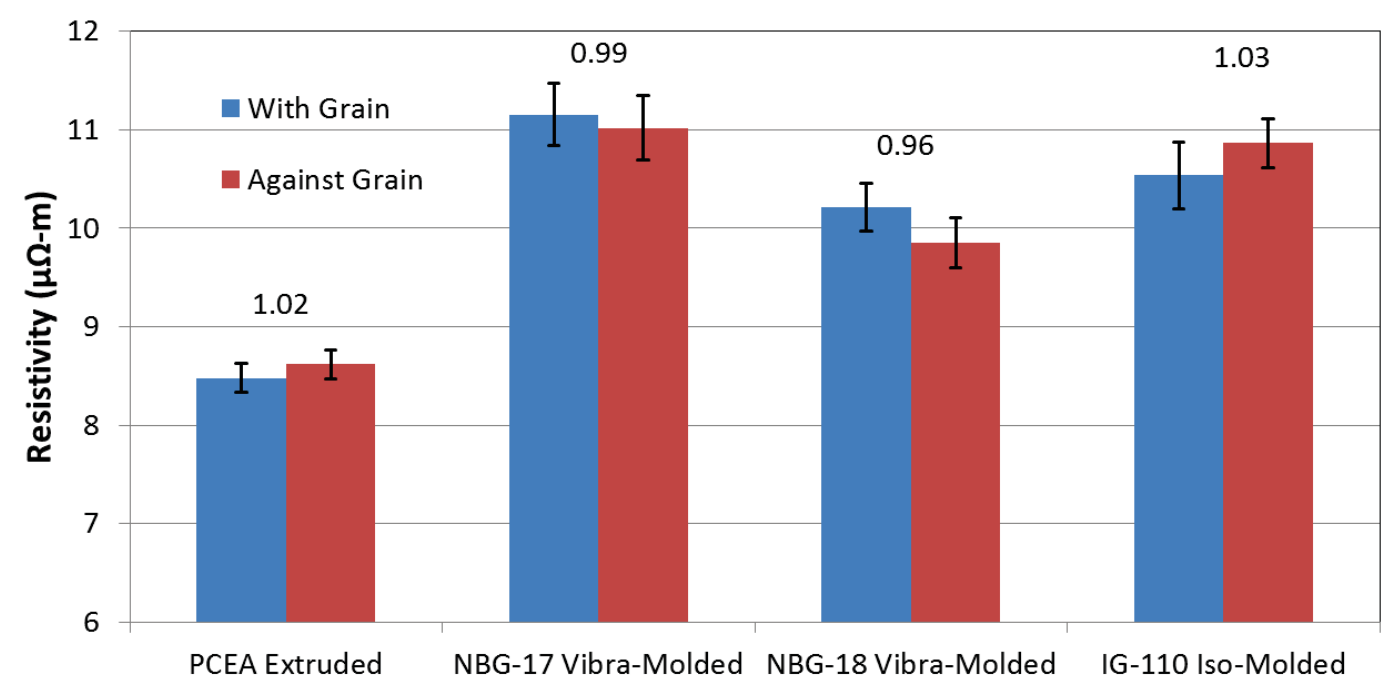

Figure 18. Electrical resistivity for several nuclear grade graphite types. The anisotropy ratio is called out above each set of data bars.

\subsection{Approximation of Elastic Modulus from the Measurement of Sonic Velocity}

Figures A-71 through A-80 are plots of Young's and shear modulus determined from the measurement of sonic velocity. Statistical parameters are shown in Table B-10 and B-11 for Young's and shear moduli, respectively. The IQR analysis does not reveal any inconsistency or outlier problems. As described in Section 4.1, the modulus is easily correlated with the density of the specimens. This known behavior results from the fact that material containing more porosity and cracks (lower density) is more compliant, and therefore will have a lower modulus of elasticity.

Table 5 shows Young's modulus measured using the sonic velocity technique. Although the COV includes potential material variability these values compare favorably to a COV of $3.8 \%$ reported in the precision and bias section of ASTM C769. Consistent with the discussion of the effects of density here, we see the highest density graphite, NBG-17, and NBG-18 produce the highest modulus.

Table 5. Young's modulus by measurement of sonic velocity.

\begin{tabular}{|l|l|l|l|l|l|}
\hline $\begin{array}{c}\text { Graphite } \\
\text { Type and } \\
\text { Process } \\
\text { Method }\end{array}$ & $\begin{array}{c}\text { Young's } \\
\text { Modulus } \\
\text { Against Grain } \\
\text { Mean (GPA) }\end{array}$ & $\begin{array}{c}\text { Against } \\
\text { Grain } \\
\text { COV \% }\end{array}$ & $\begin{array}{c}\text { Young's } \\
\text { Modulus with } \\
\text { Grain Mean } \\
\text { (GPA) }\end{array}$ & $\begin{array}{c}\text { With Grain } \\
\text { COV \% }\end{array}$ & $\begin{array}{c}\text { Young's Modulus } \\
\text { Anisotropy Ratio }\end{array}$ \\
\hline $\begin{array}{l}2114 \\
\text { Iso-Molded }\end{array}$ & 12.10 & 2.58 & & NA \\
\hline $\begin{array}{l}\text { IG-110 } \\
\text { Iso-Molded }\end{array}$ & 10.89 & 2.15 & 10.17 & 3.52 & 1.07 \\
\hline $\begin{array}{l}\text { NBG-17 } \\
\text { Vibra- } \\
\text { Molded }\end{array}$ & 15.03 & 4.00 & 15.06 & 0.82 & 1.00 \\
\hline
\end{tabular}




\begin{tabular}{|l|l|l|l|l|l|}
\hline $\begin{array}{c}\text { Graphite } \\
\text { Type and } \\
\text { Process } \\
\text { Method }\end{array}$ & $\begin{array}{c}\text { Young's } \\
\text { Modulus } \\
\text { Against Grain } \\
\text { Mean (GPA) }\end{array}$ & $\begin{array}{c}\text { Against } \\
\text { Grain } \\
\text { COV \% }\end{array}$ & $\begin{array}{c}\text { Young's } \\
\text { Modulus with } \\
\text { Grain Mean } \\
\text { (GPA) }\end{array}$ & $\begin{array}{c}\text { With Grain } \\
\text { COV \% }\end{array}$ & $\begin{array}{c}\text { Young's Modulus } \\
\text { Anisotropy Ratio }\end{array}$ \\
\hline $\begin{array}{l}\text { NBG-18 } \\
\text { Vibra- } \\
\text { Molded }\end{array}$ & 16.05 & 1.74 & 15.52 & 1.13 & 1.03 \\
\hline $\begin{array}{l}\text { PCEA } \\
\text { Extruded }\end{array}$ & 12.12 & 1.63 & 12.30 & 1.71 & 0.99 \\
\hline
\end{tabular}

\subsection{Modulus of Elasticity by Measurement of Fundamental Frequency}

Young's modulus determined by measurement of fundamental frequency is plotted in Figures A-61 through A-65 and the statistical data is contained in Table B-8. Statistically this data is well-behaved with the IQR analysis showing no problems with outliers. Table 6 shows a comparison of Young's modulus from the measurement of fundamental frequency for the primary grades of graphite. The COVs for all compare favorably with $\sim 3 \%$ uncertainty in the measurement technique.

Table 6 also shows that the new grades of graphite being tested exhibit very good isotropy in modulus. Note that for grade 2114 only, specimens were machined in what was defined as the against grain direction due to the geometry of the feed stock material.

Table 6. Young's modulus by measurement of fundamental frequency.

\begin{tabular}{|l|l|l|l|l|l|}
\hline $\begin{array}{l}\text { Graphite } \\
\text { Type and } \\
\text { Process } \\
\text { Method }\end{array}$ & $\begin{array}{c}\text { Young's } \\
\text { Modulus } \\
\text { Against Grain } \\
\text { Mean (GPA) }\end{array}$ & $\begin{array}{c}\text { Against } \\
\text { Grain } \\
\text { COV \% }\end{array}$ & $\begin{array}{c}\text { Young's } \\
\text { Modulus with } \\
\text { Grain Mean } \\
\text { (GPA) }\end{array}$ & $\begin{array}{c}\text { With Grain } \\
\text { COV \% }\end{array}$ & $\begin{array}{c}\text { Young's Modulus } \\
\text { Anisotropy Ratio }\end{array}$ \\
\hline $\begin{array}{l}2114 \\
\text { Isomolded }\end{array}$ & 10.56 & 1.99 & & NA \\
\hline $\begin{array}{l}\text { IG-110 } \\
\text { Iso-Molded }\end{array}$ & 9.72 & 2.53 & 9.28 & 3.17 & 1.05 \\
\hline $\begin{array}{l}\text { NBG-17 } \\
\text { Vibra- } \\
\text { Molded }\end{array}$ & 11.74 & 3.48 & 11.57 & 0.89 & 1.01 \\
\hline $\begin{array}{l}\text { NBG-18 } \\
\text { Vibra- } \\
\text { Molded }\end{array}$ & 12.35 & 1.52 & 11.80 & 0.57 & 1.05 \\
\hline $\begin{array}{l}\text { PCEA } \\
\text { Extruded }\end{array}$ & 9.81 & 2.35 & 9.09 & 3.31 & 1.08 \\
\hline
\end{tabular}




\subsection{Thermal Expansion}

Mean coefficient of thermal expansion data is plotted in Figures A-89 through A-112 . A statistical evaluation of the CTE data was performed at three discrete temperatures for each graphite type: $100^{\circ} \mathrm{C}$, $500^{\circ} \mathrm{C}$, and $900^{\circ} \mathrm{C}$. Again, the dashed lines in these plots indicate the upper and lower IQR limits, and there are no outliers to consider. Tables B-5 through B-7 contain the mean, standard deviation, and COV values for the data evaluated at the discrete temperatures. With the exception of NBG-18 and IG-110, all COVs are below or on the order of the $\pm 3 \%$ measurement uncertainty when calculated for the with and against grain specimen groups individually. The relatively large COVs of NGB-18 and IG-110 are a result of measurement equipment differences. In both cases, the against grain specimens were measured at INL and the with grain specimens were measure at ORNL. These measurement differences are directly visible in Figures A-45 and A-53.

Figure 19 shows the average of all specimens for several of the primary grades of graphite with error bars indicating \pm 1 standard deviation. All increase with temperature in a near linear fashion between $100^{\circ} \mathrm{C}$ and $1000^{\circ} \mathrm{C}$. This type of data is useful and necessary for specifying the type of graphite used in future reactor designs. The $\sim 25 \%$ difference between NBG-17 and IG-110 could result in significant differences stresses that arise from thermal gradients. These differences could influence graphite grade choices.

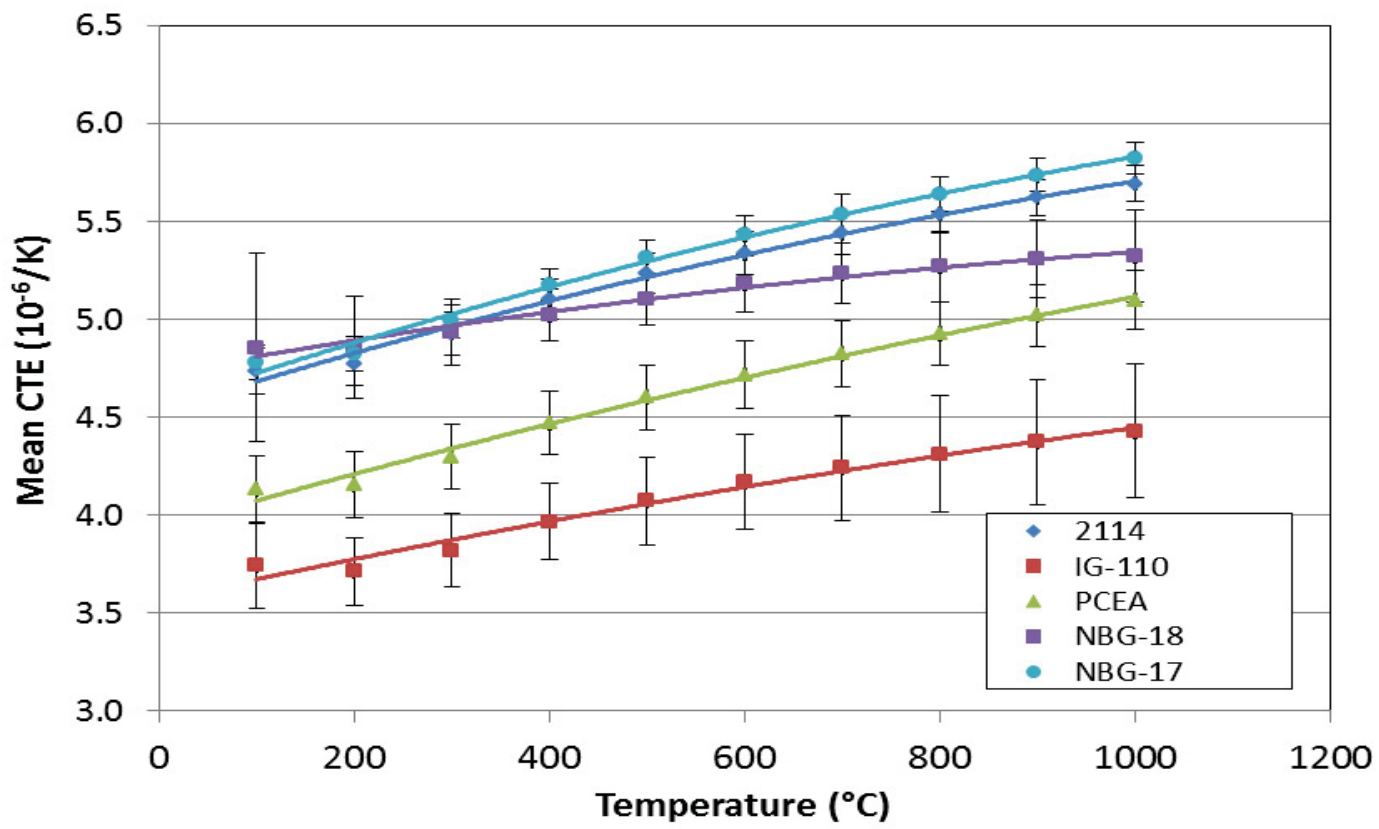

Figure 19. Mean CTE for several types of nuclear grade graphite as a function of temperature. Error bars represent \pm 1 standard deviation in the data that was used to obtain the averages plotted.

Measurements of CTE were performed on both with grain and against grain specimens. Figure 20 shows the CTE anisotropy ratio for the same primary grades of graphite as a function of temperature. Graphite grade 2114 is not included because specimens were only fabricated in the against grain direction. The trend with temperature for both IG-110 and NBG-18 is a result of measurement differences between INL and ORNL discussed above. Based on previous data [19], the isotropy ratios for IG-110 and NBG-18 are $\sim 1.01$ and $\sim 0.98$, respectively, and are constant with temperature as are the ratios for PCEA and NBG-17. 


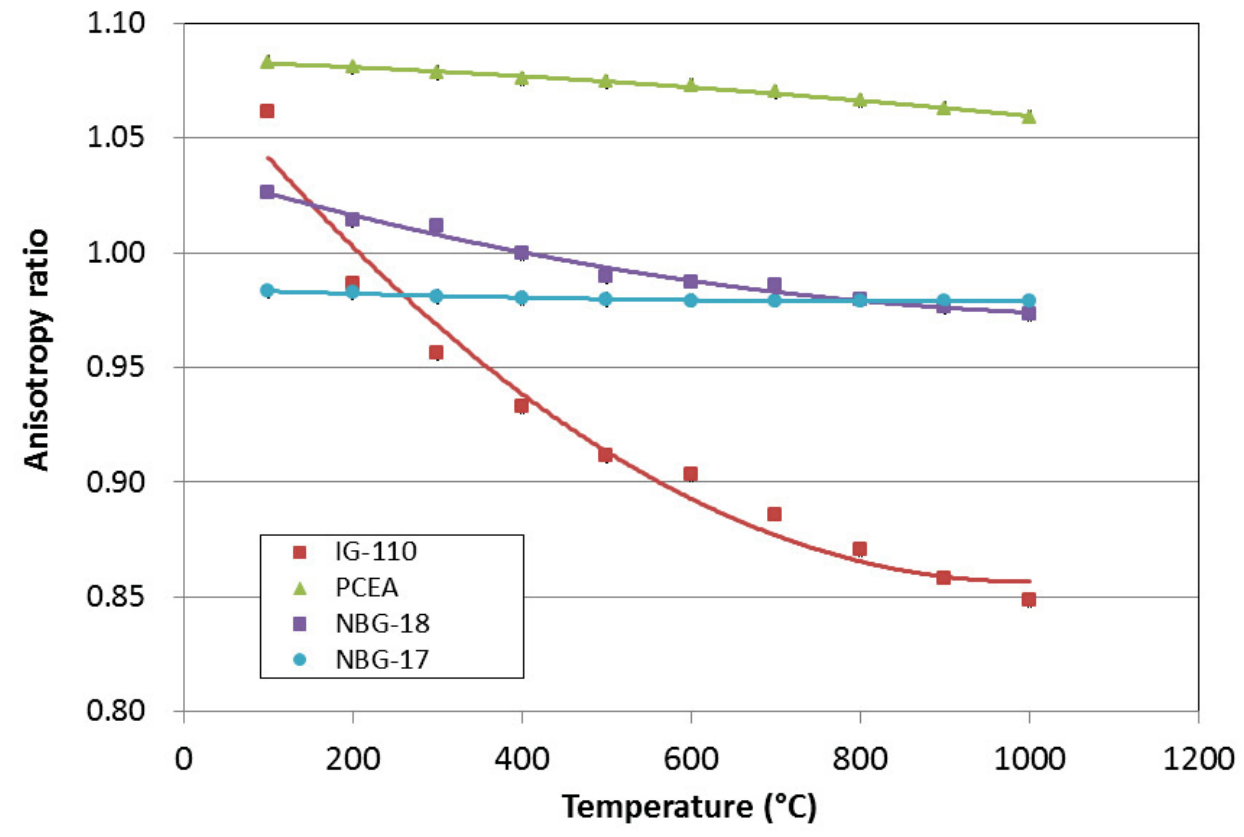

Figure 20. CTE anisotropy ratio for several types of nuclear grade graphite as a function of temperature.

\subsection{Thermal Diffusivity}

Plots of thermal diffusivity are shown in Figures A-111 through A-126. As with the CTE data, discrete temperatures of $100^{\circ} \mathrm{C}, 500^{\circ} \mathrm{C}$, and $1000^{\circ} \mathrm{C}$ were statistically evaluated. Tables B- 16 through B-18 contain values of the mean, standard deviation and COV. The COVs for all graphite types are in the neighborhood of the specified $\pm 3 \%$ uncertainty of the measurement technique. Mainly, the diffusivity data is well-behaved with only 3 data points of the 2114 grade that somewhat exceed the IQR limits and again are most likely a result of slight material variability.

Figure 21 shows the average thermal diffusivity for the five primary graphite grades of interest. Error bars are \pm 1 standard deviation and in many cases are smaller than the plotted symbol. The diffusivity of the different grades of graphite varies as much as $40 \%$ at room temperature, but only $\sim 15 \%$ at $1000^{\circ} \mathrm{C}$. As seen in the coefficient of thermal expansion, these differences in diffusivity are significant and will need to be considered in the choice of graphite's for future reactor designs.

Shown in Figure 22 is the anisotropy ratio for the same graphite grades. The anisotropy for all are constant with temperature and as with the other properties, the current grades of graphite show a high degree of isotropy for thermal diffusivity. 


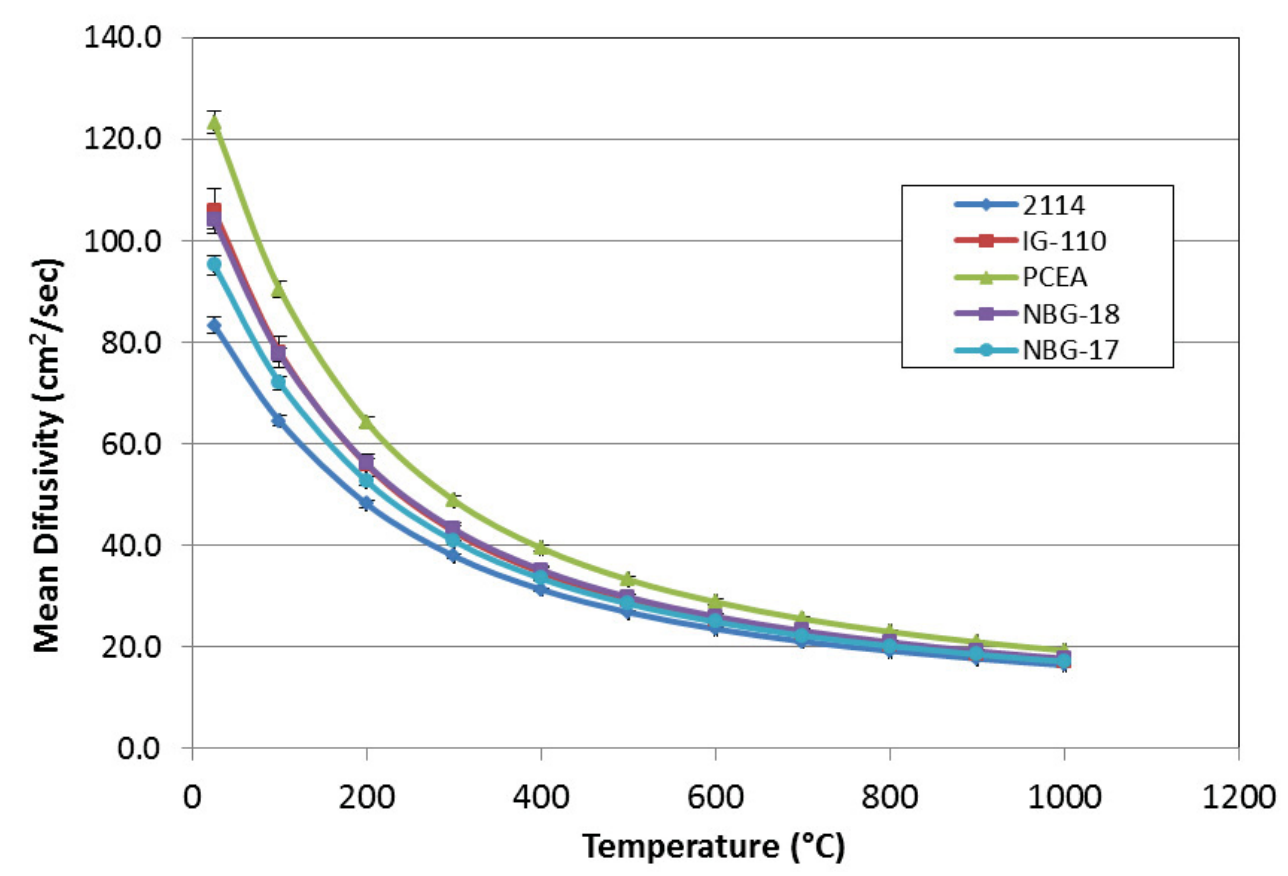

Figure 21. Thermal diffusivity for various graphite types as a function of temperature. Error bars are 1 standard deviation in the data and in some cases smaller than the data symbol.

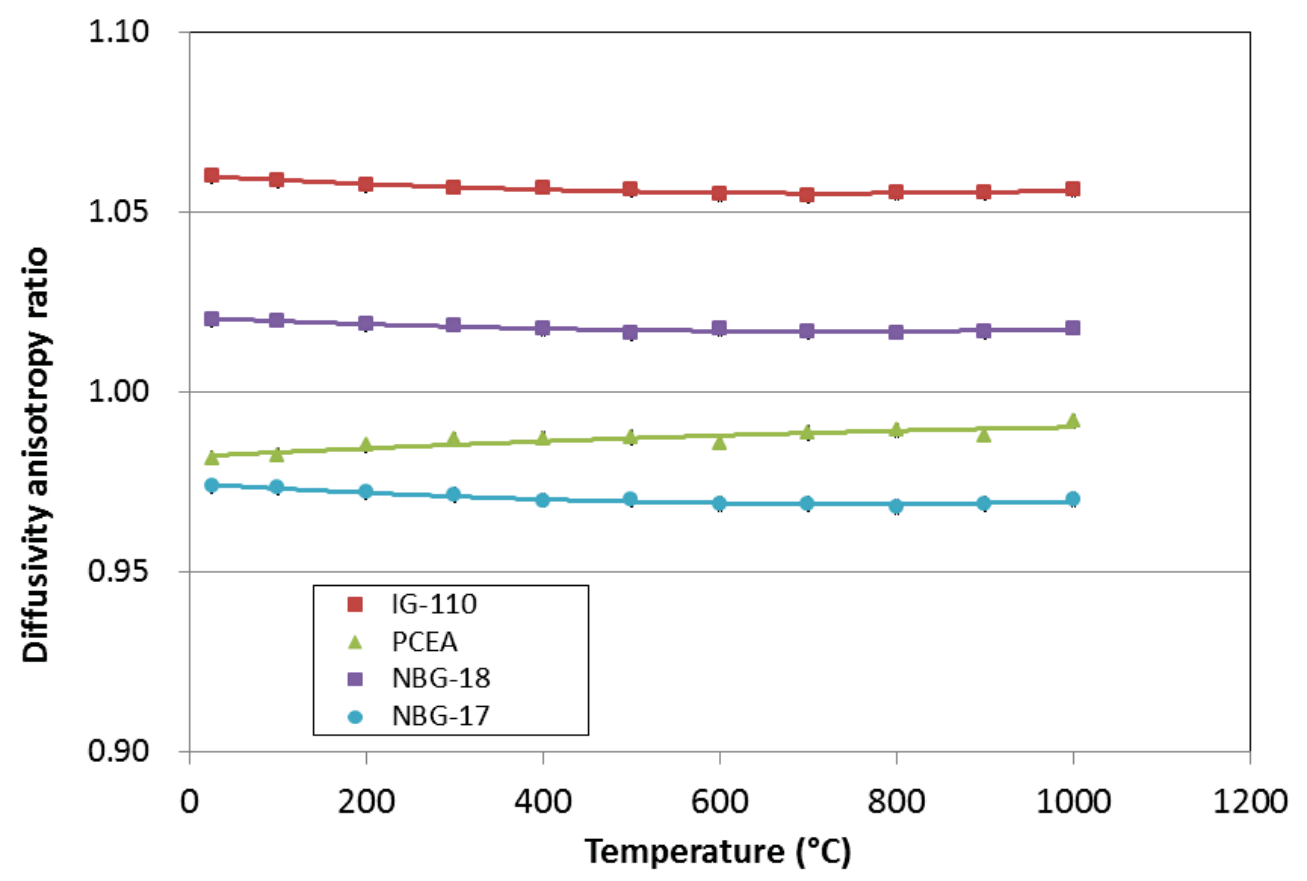

Figure 22. Thermal diffusivity anisotropy ratio for several types of nuclear grade graphite as a function of temperature. 


\section{Appendix A AGC-3 Pre-irradiation Data}

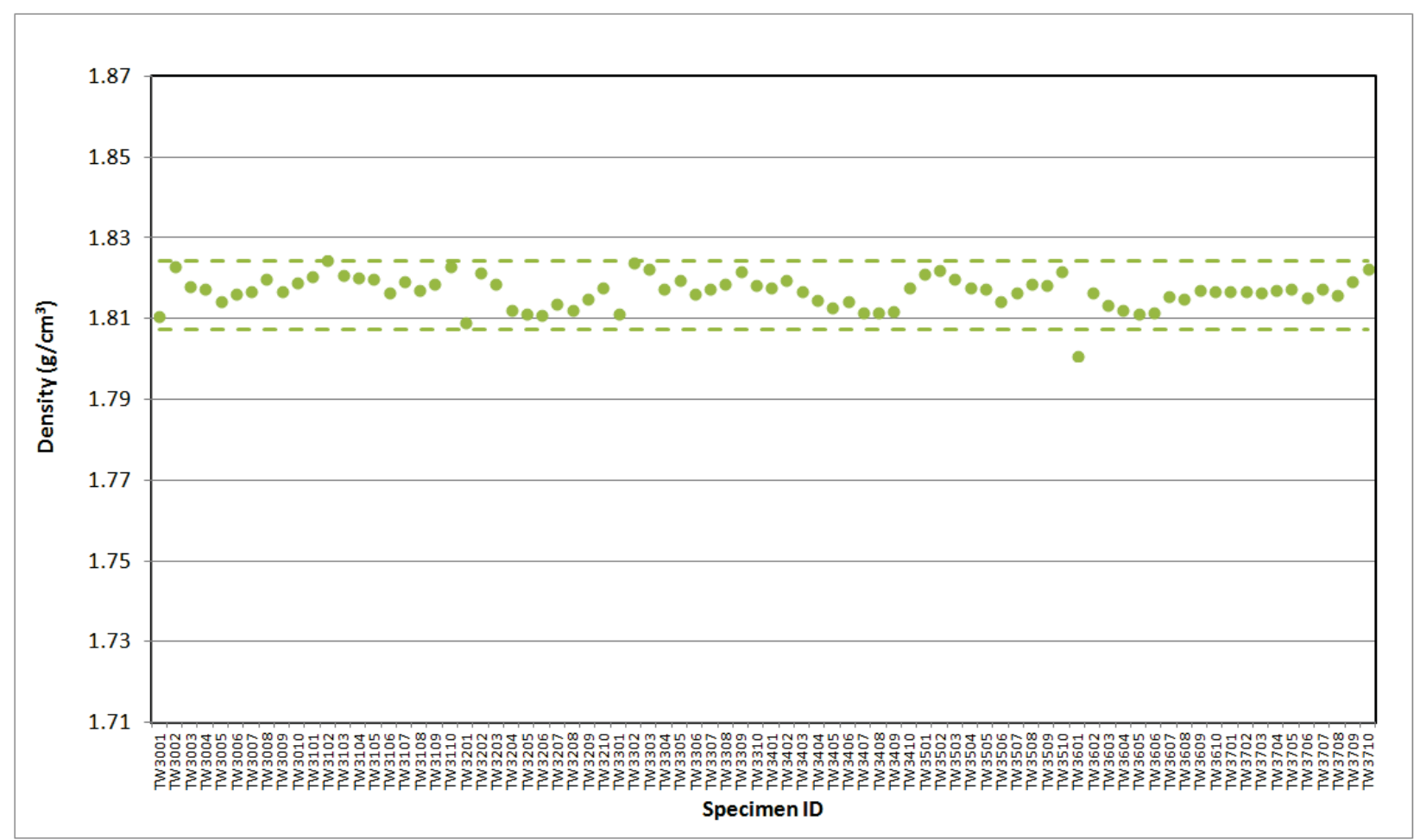

Figure A-1. 2114 Creep Pre Thermal Measurement Density. 


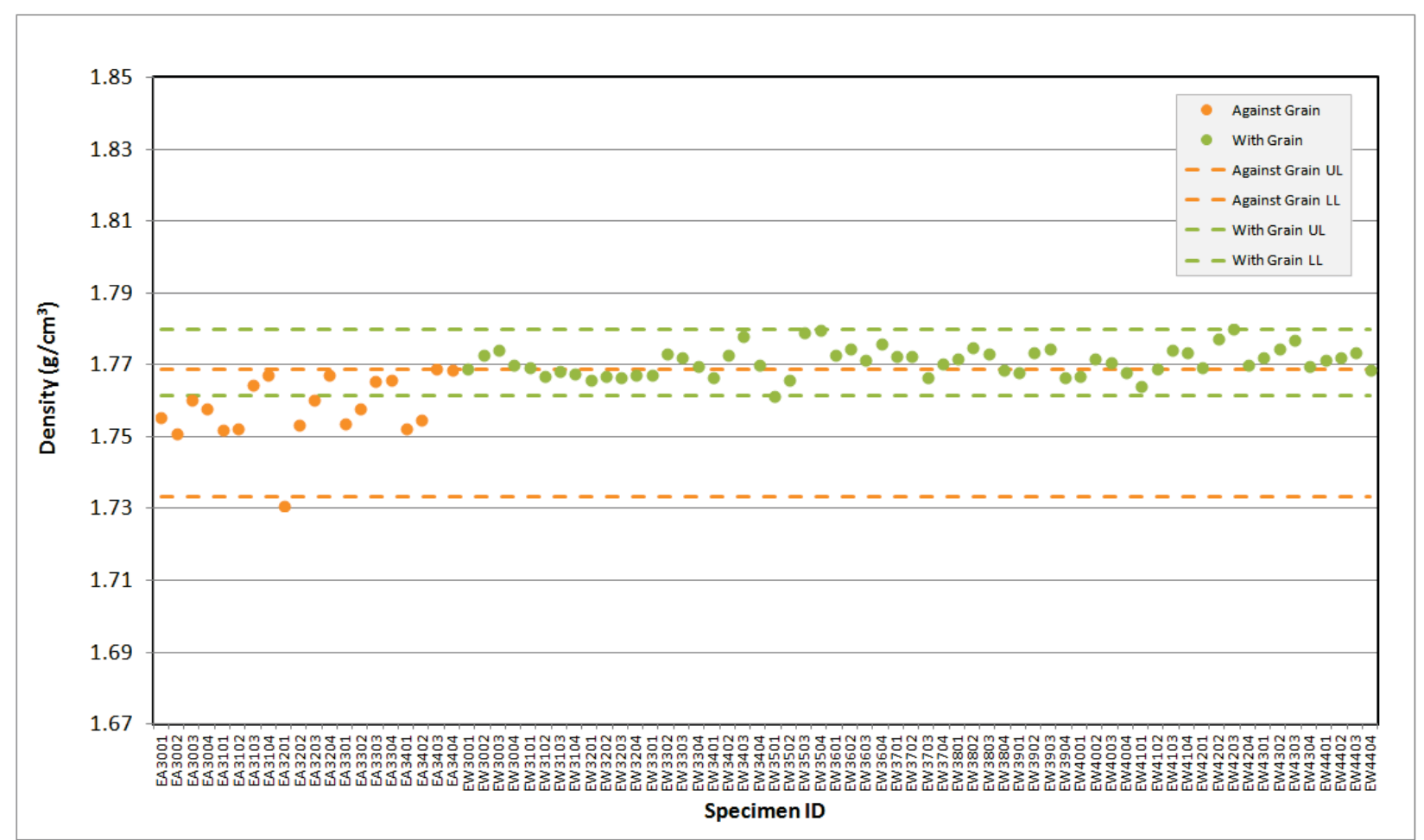

Figure A-2. IG-110 Creep Pre Thermal Measurement Density.

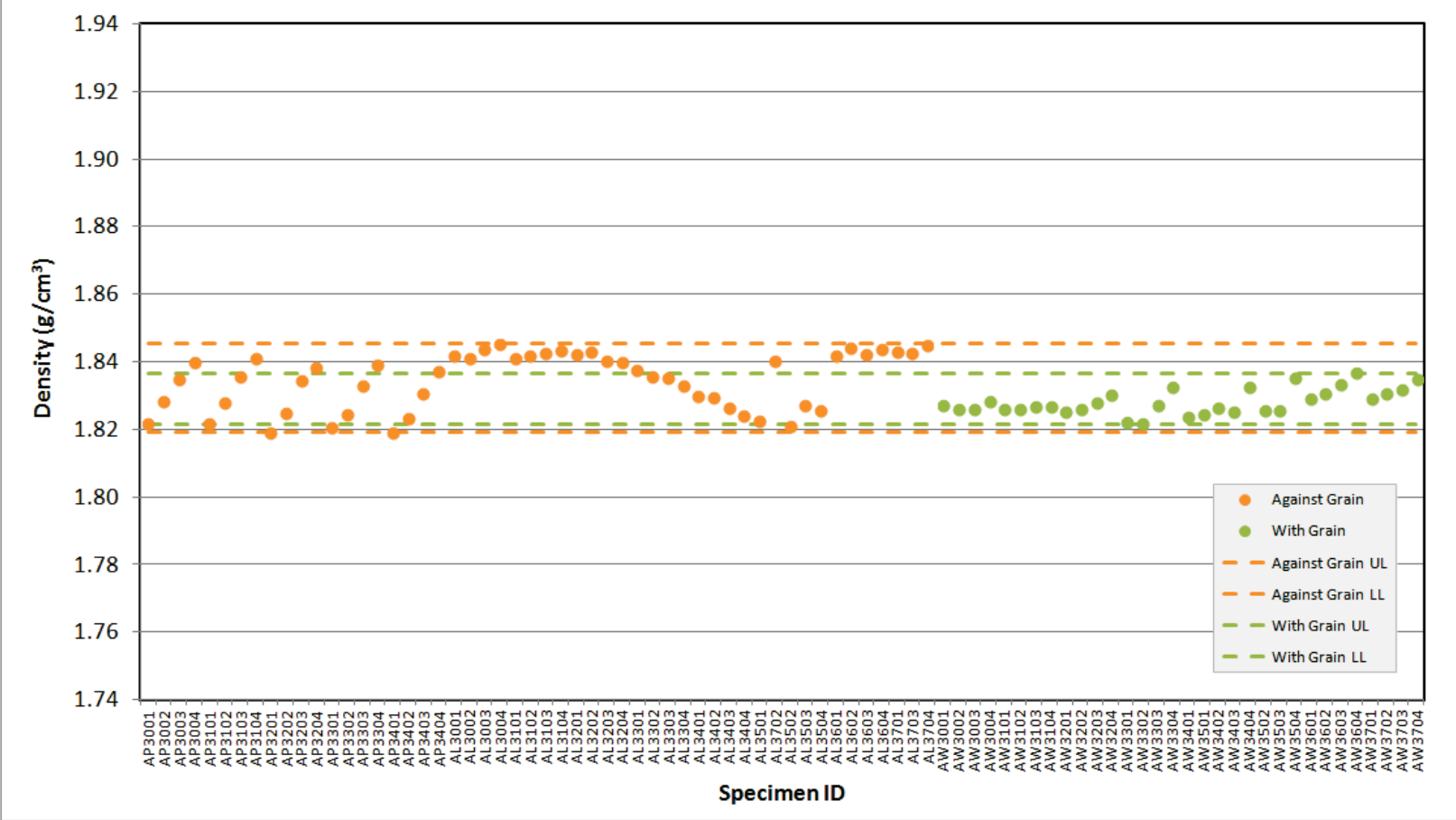

Figure A-3. NBG-17 Creep Pre Thermal Measurement Density. 


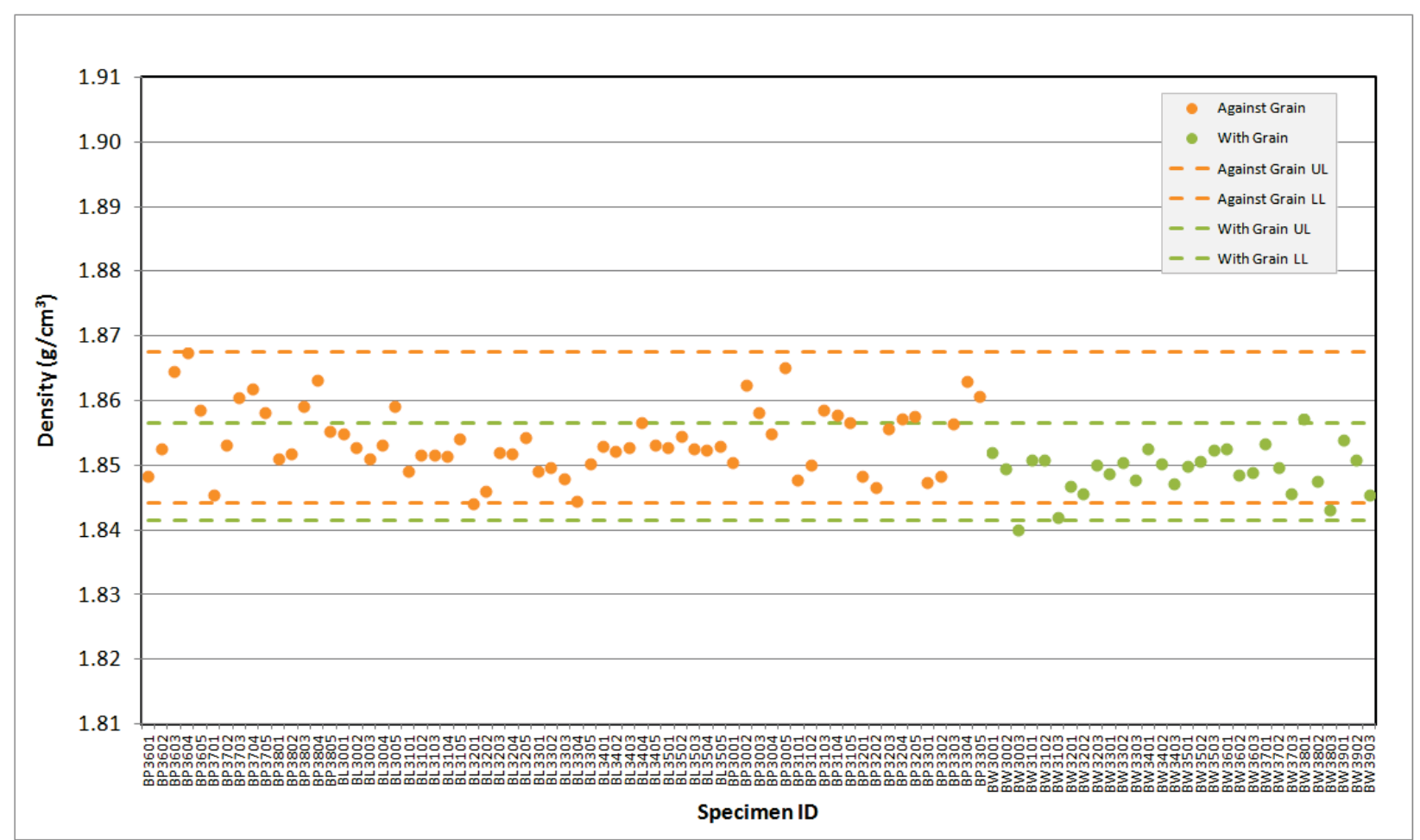

Figure A-4. NBG-18 Creep Pre Thermal Measurement Density.

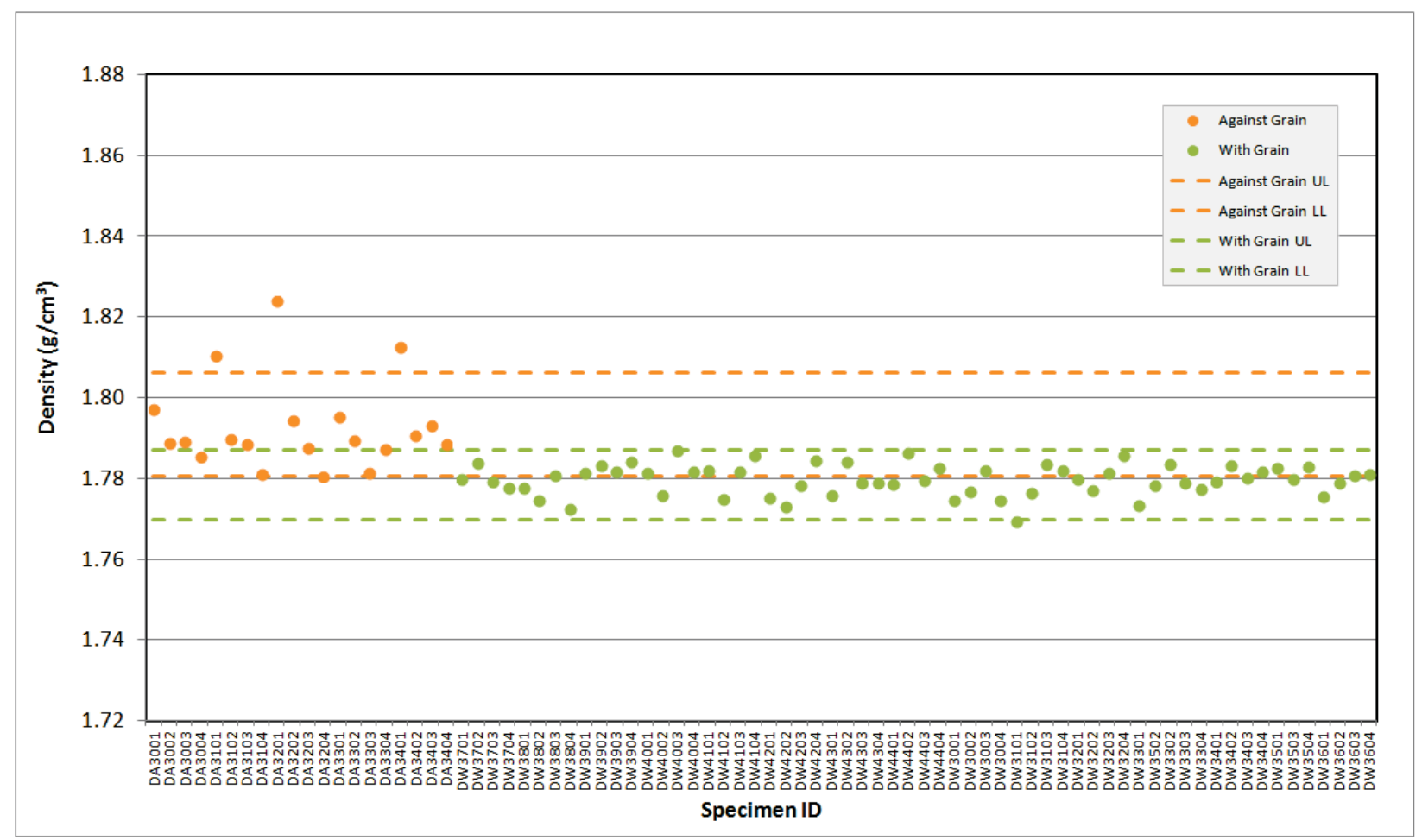

Figure A-5. PCEA Creep Pre Thermal Measurement Density. 


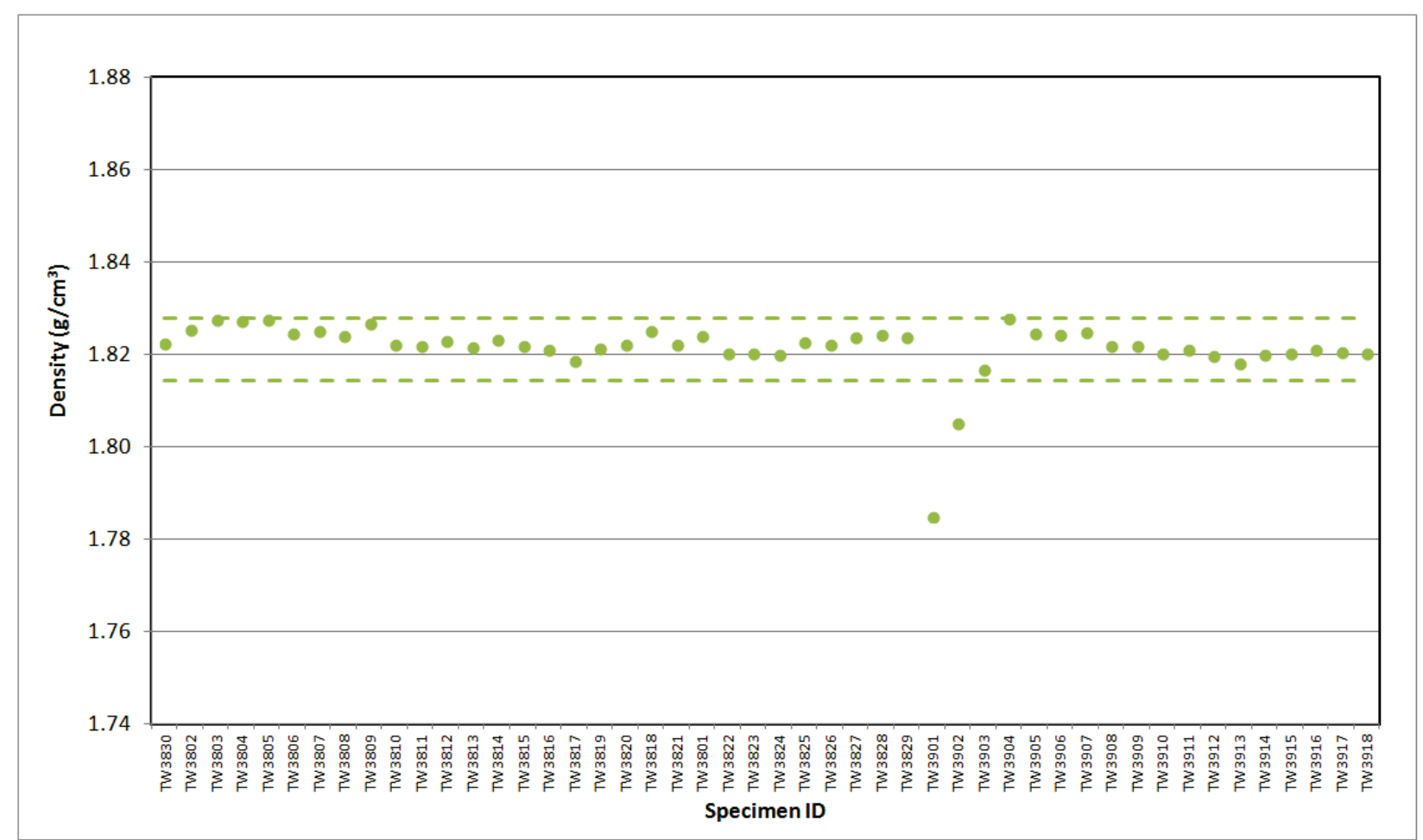

Figure A-6. 2114 Piggyback Pre Thermal Measurement Density.

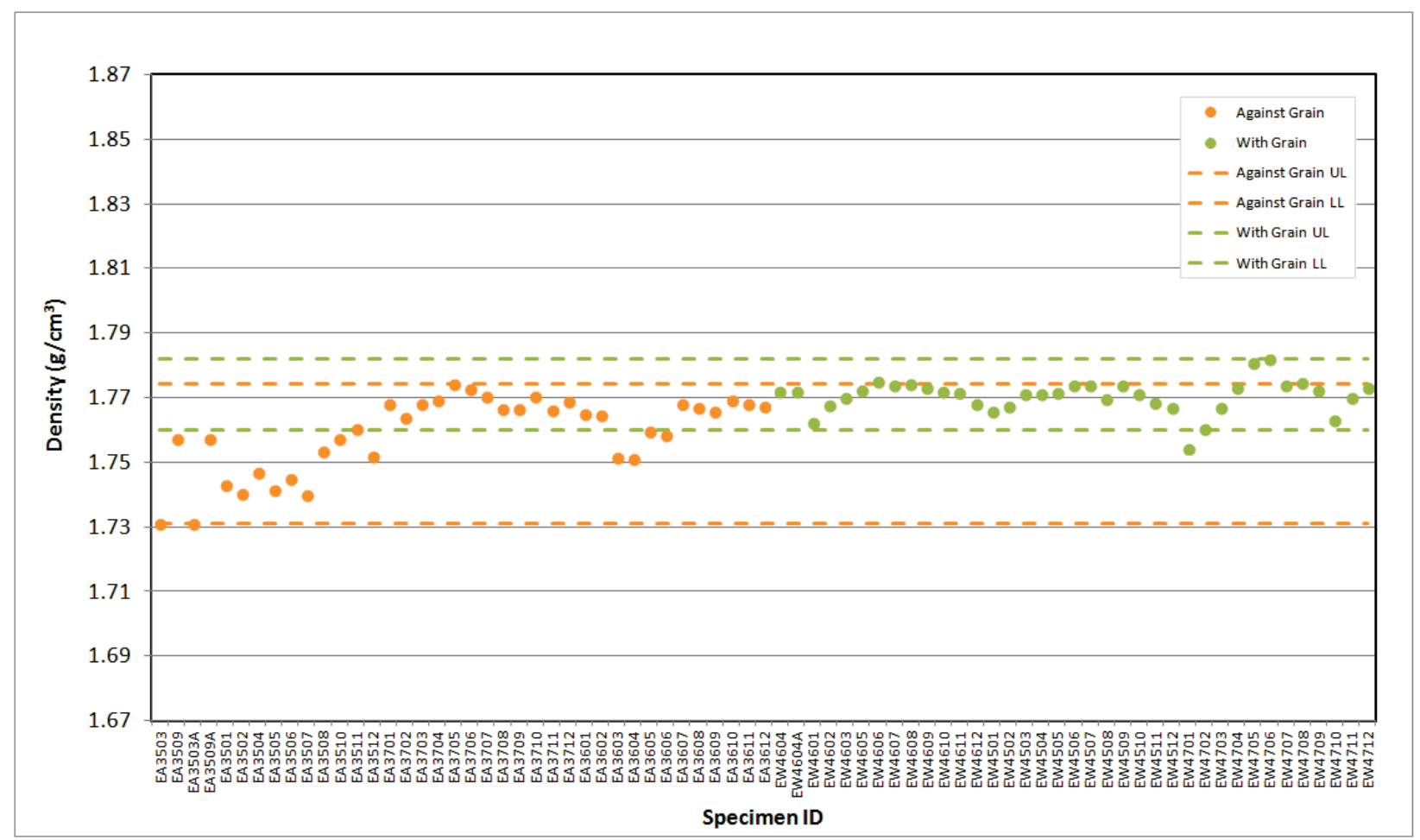

Figure A-7. IG-110 Piggyback Pre Thermal Measurement Density. 


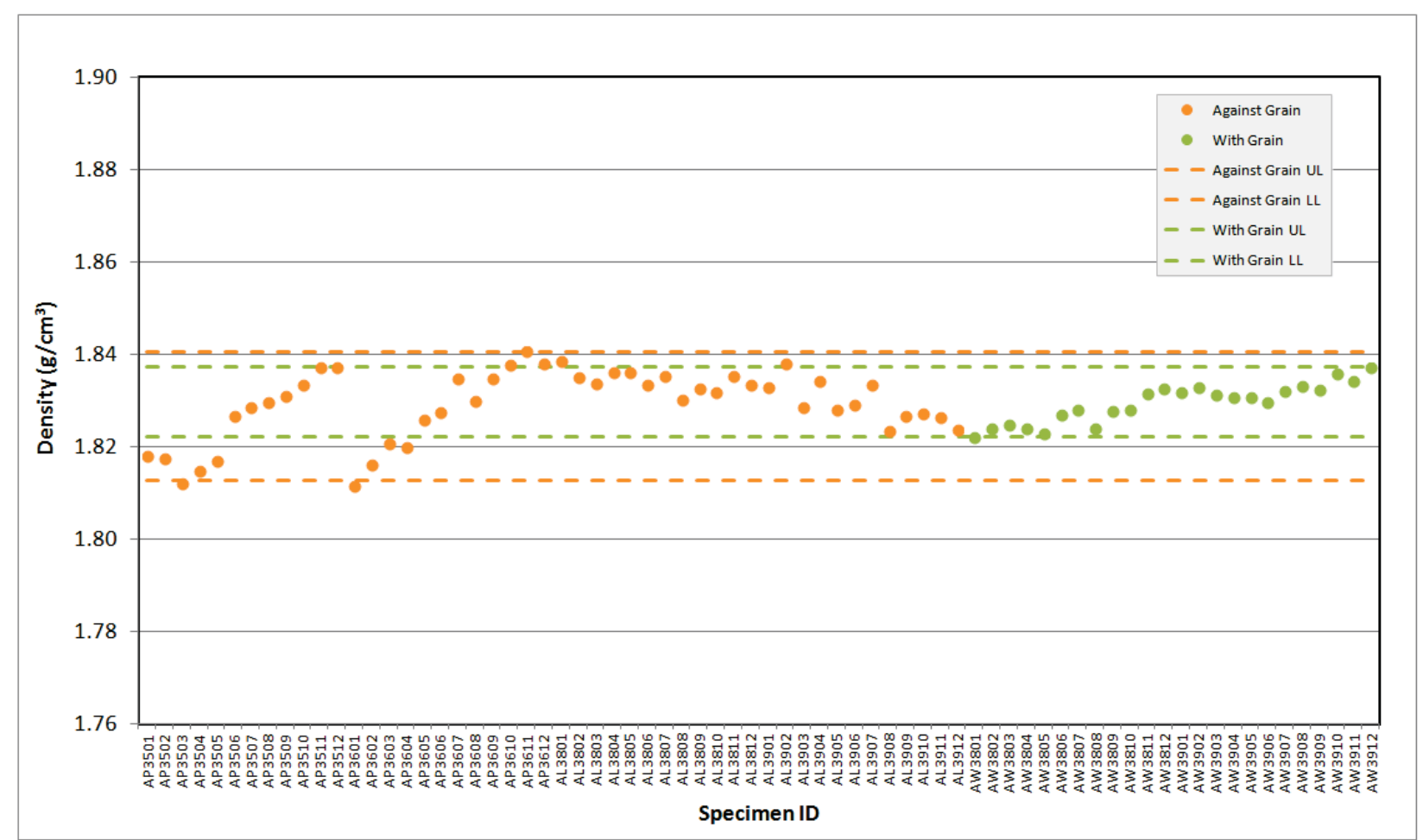

Figure A-8. NBG-17 Piggyback Pre Thermal Measurement Density.

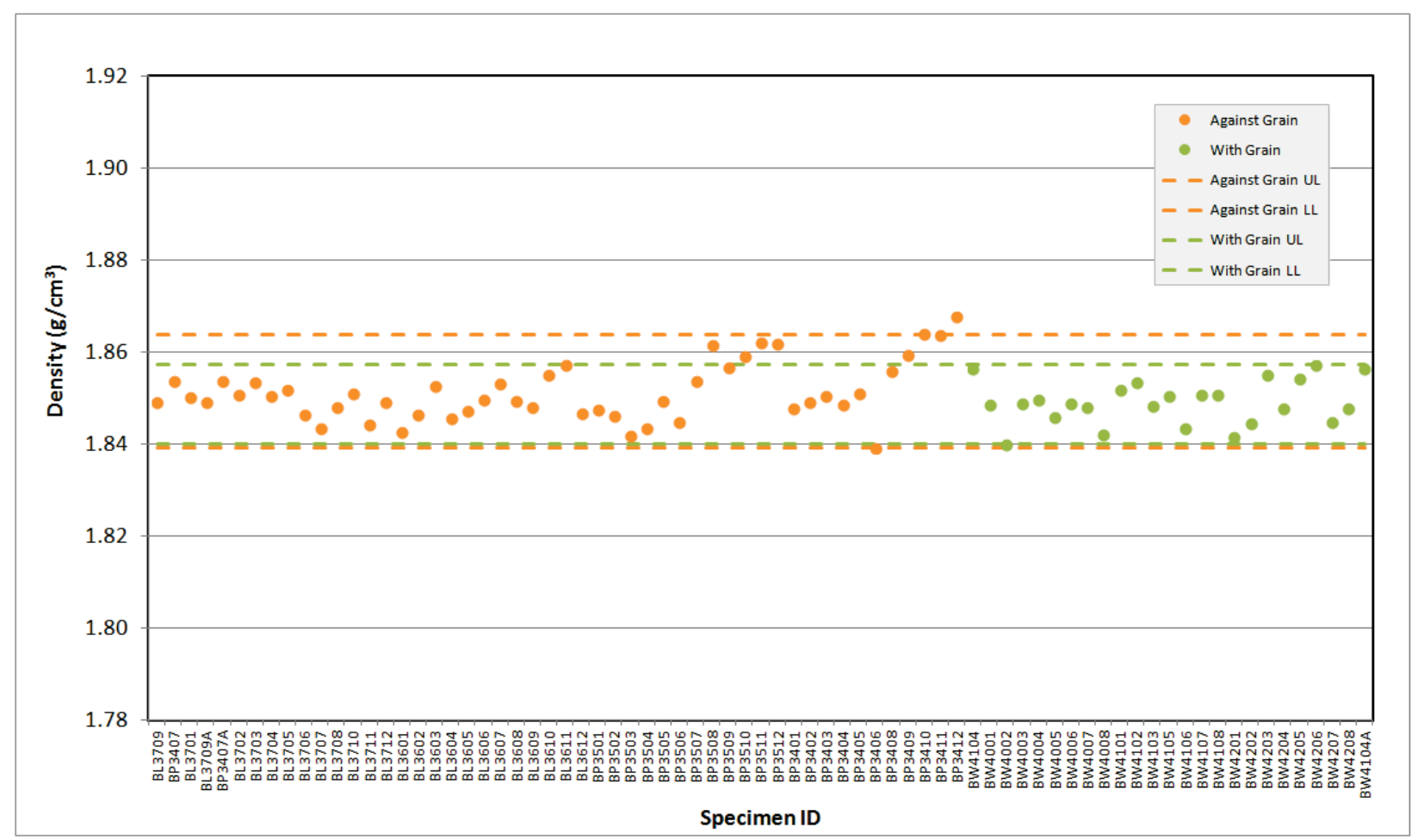

Figure A-9. NBG-18 Piggyback Pre Thermal Measurement Density. 


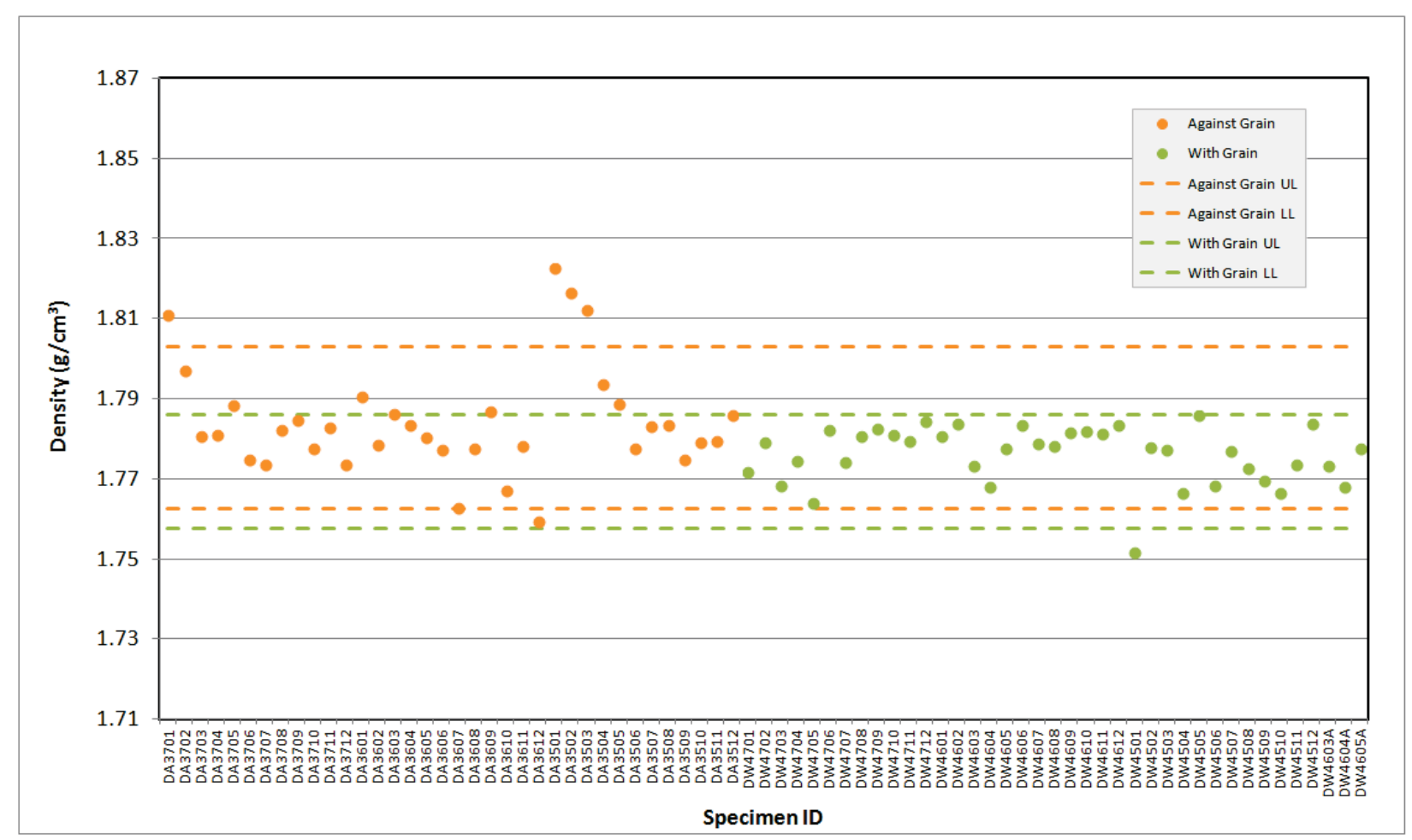

Figure A-10. PCEA Piggyback Pre Thermal Measurement Density.

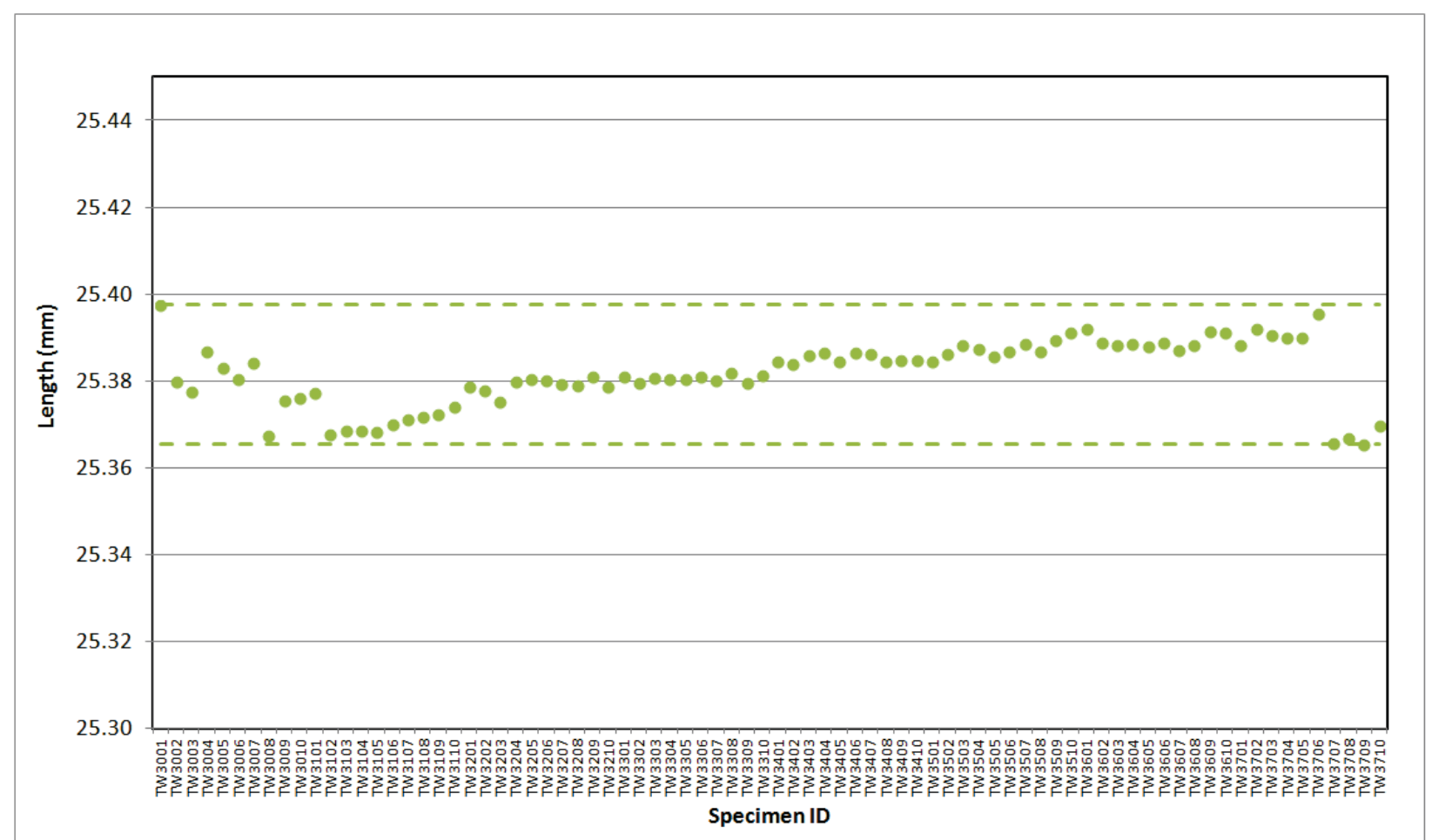

Figure A-11. 2114 Creep Pre Thermal Measurement Length. 


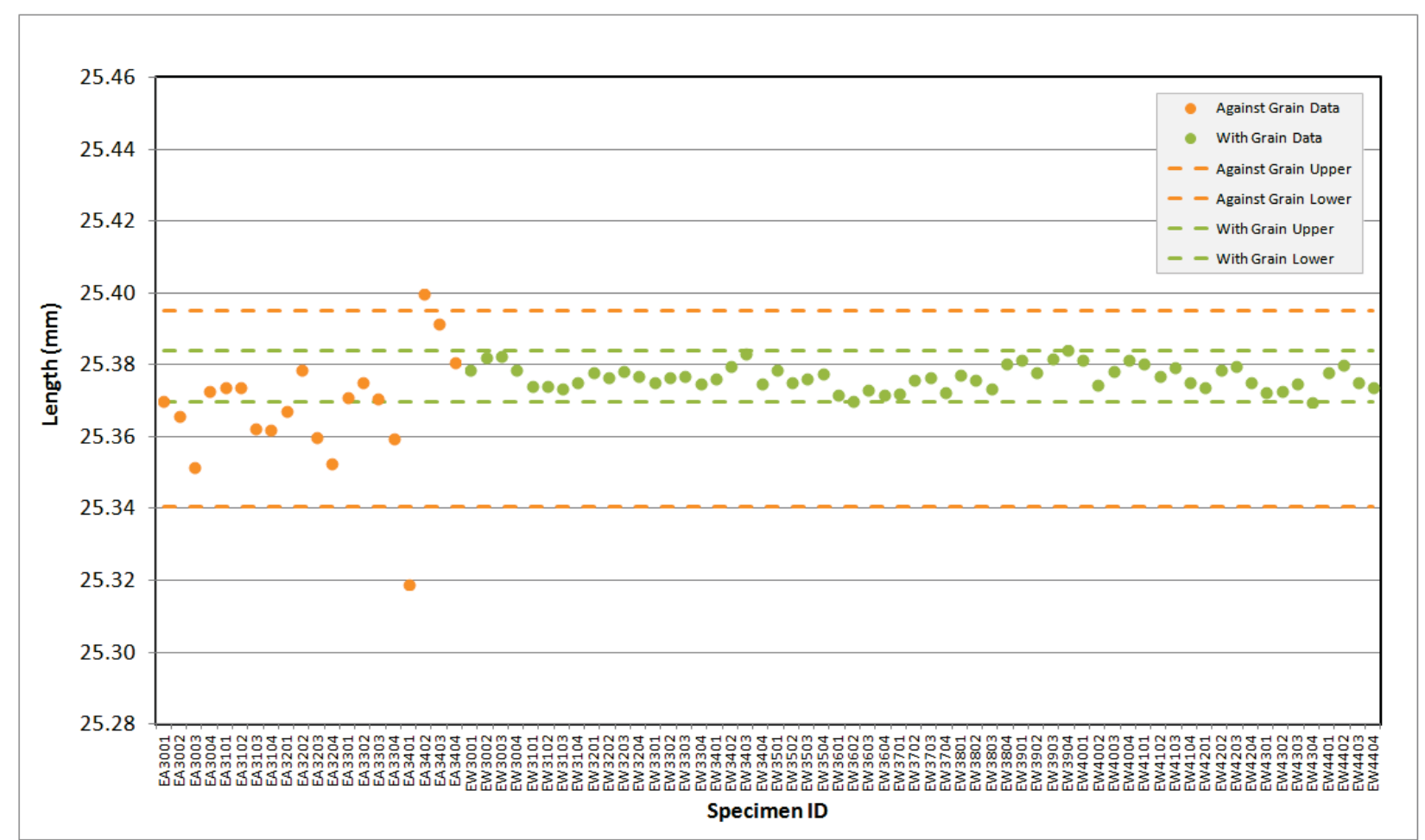

Figure A-12. IG-110 Creep Pre Thermal Measurement Length.

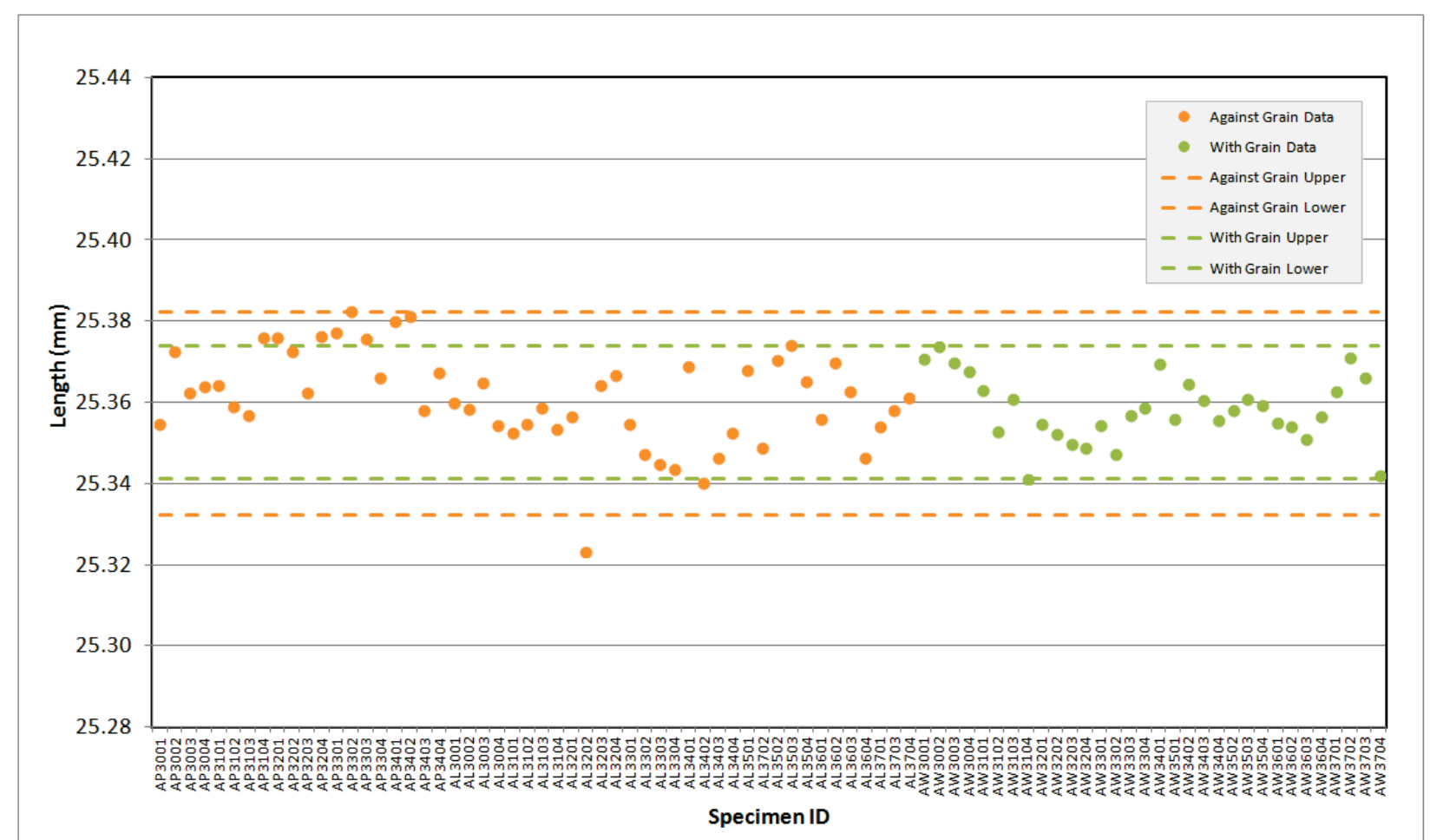

Figure A-13. NBG-17 Creep Pre Thermal Measurement Length. 


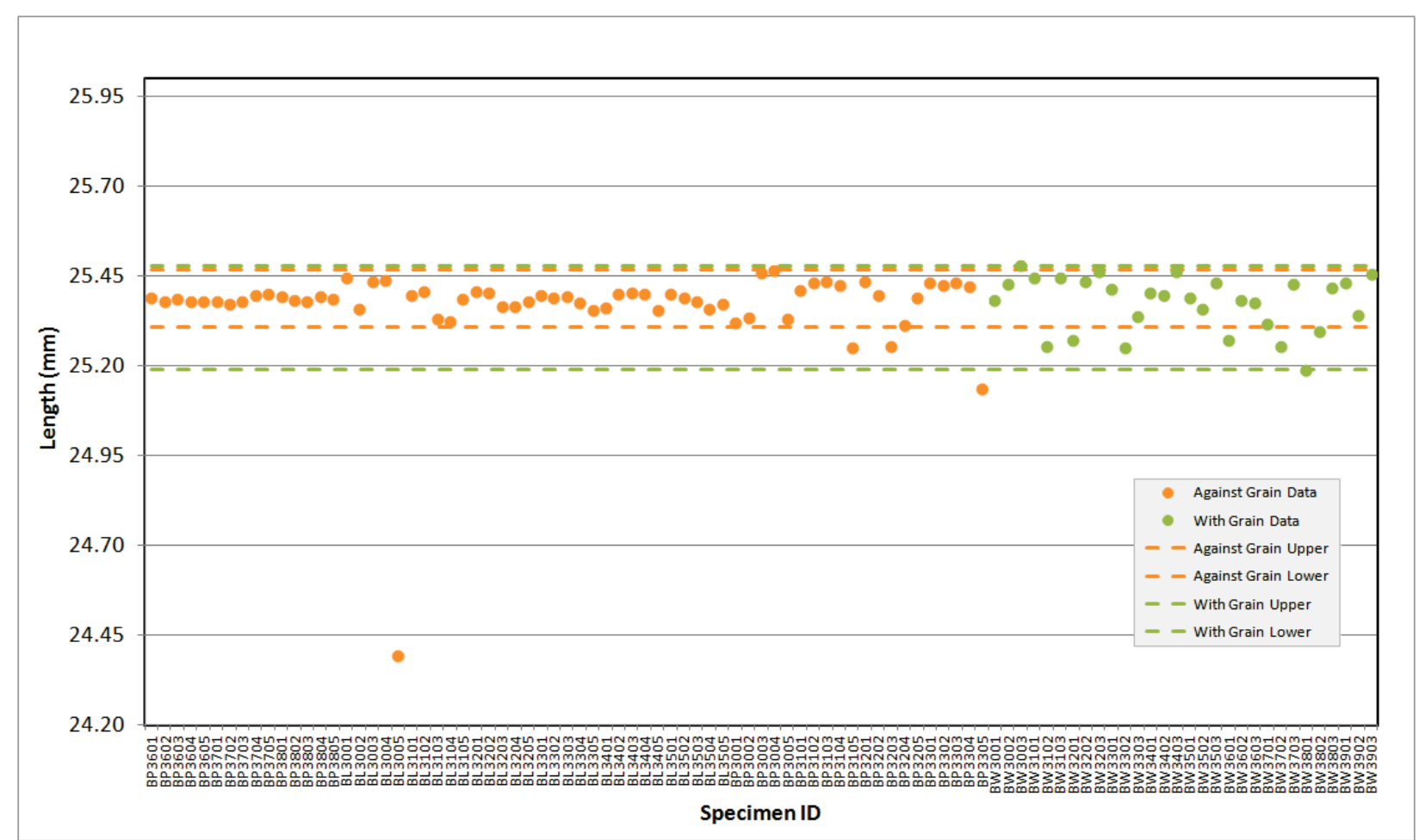

Figure A-14. NBG-18 Creep Pre Thermal Measurement Length.

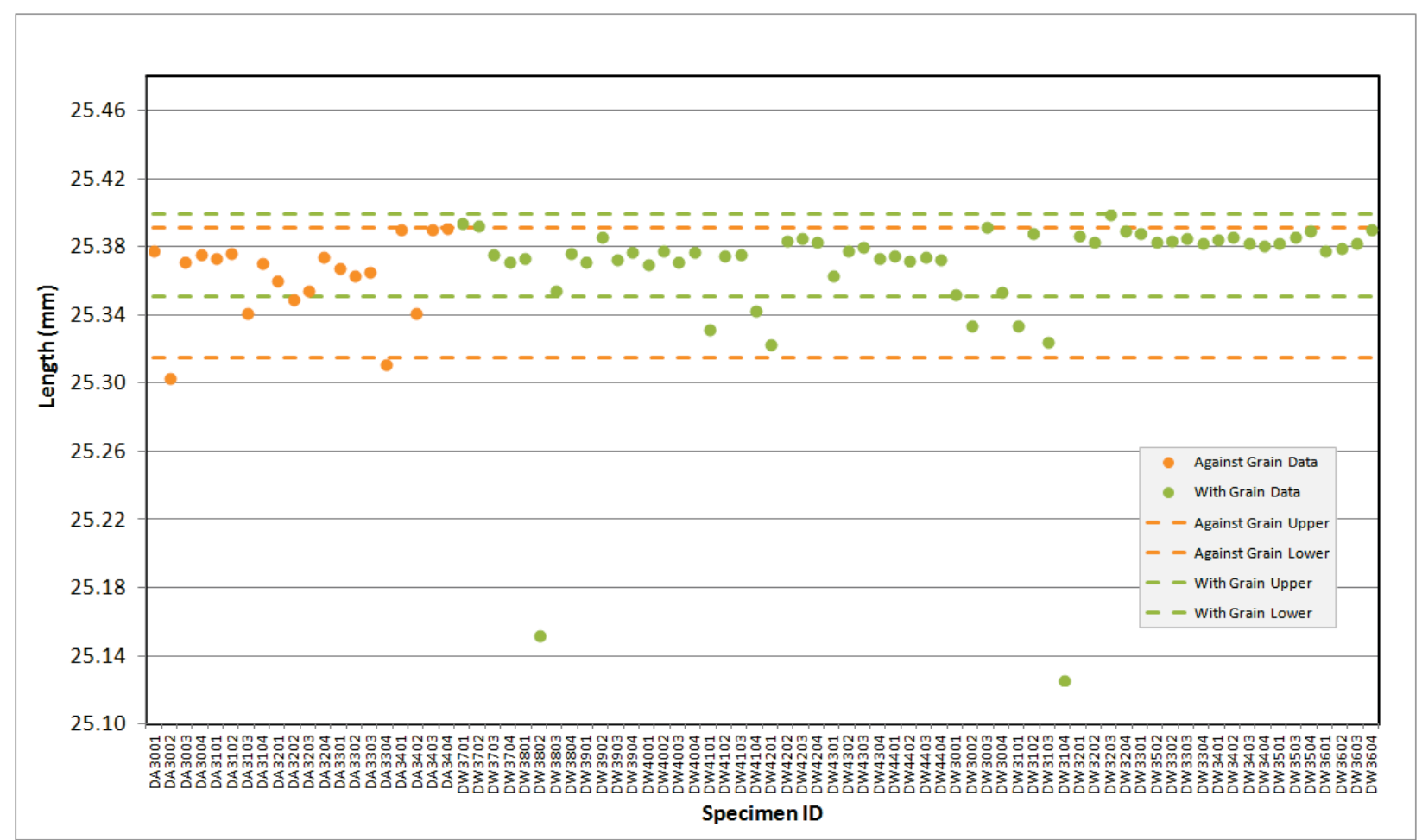

Figure A-15. PCEA Creep Pre Thermal Measurement Length. 


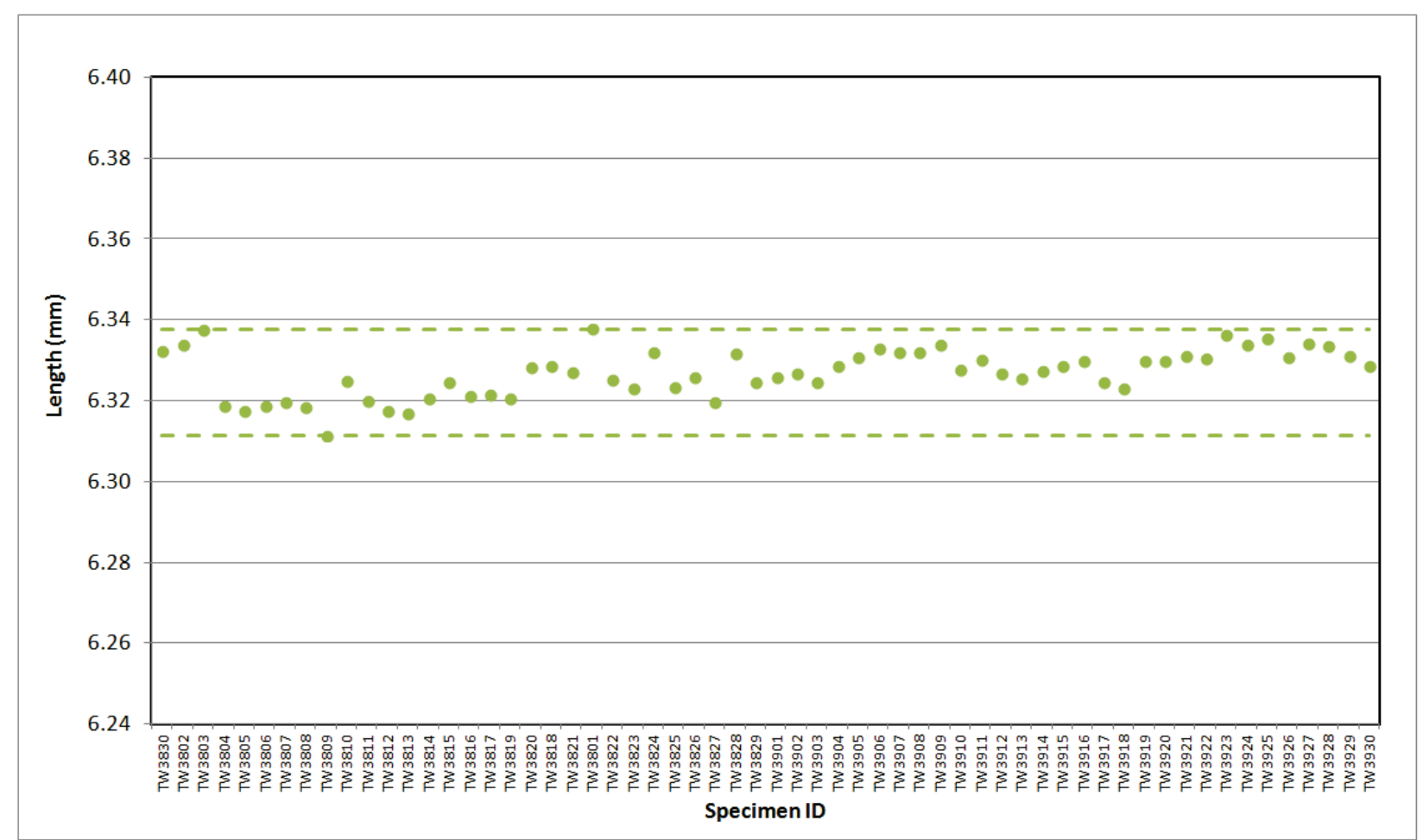

Figure A-16. 2114 Piggyback Pre Thermal Measurement Length.

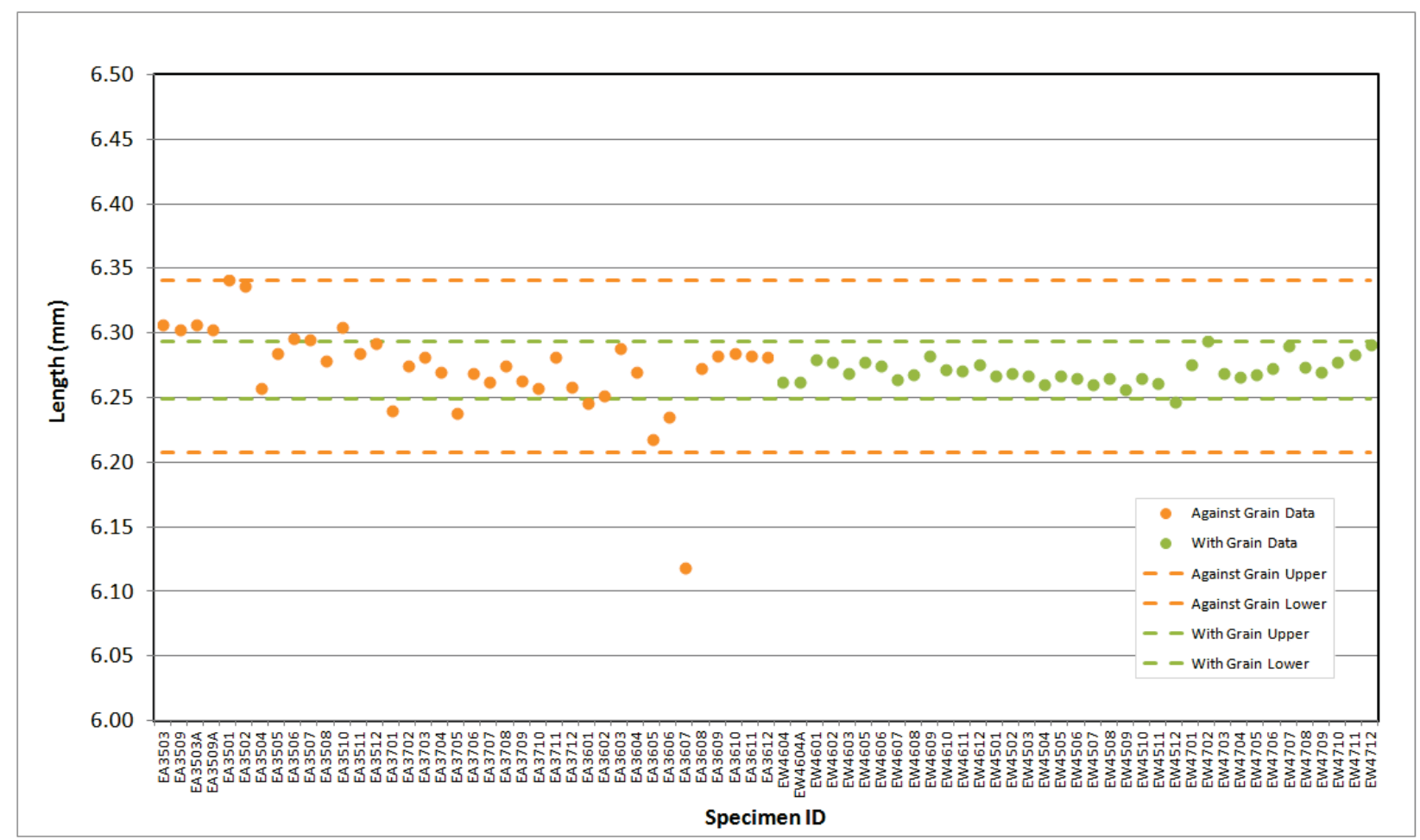

Figure A-17. IG-110 Piggyback Pre Thermal Measurement Length. 


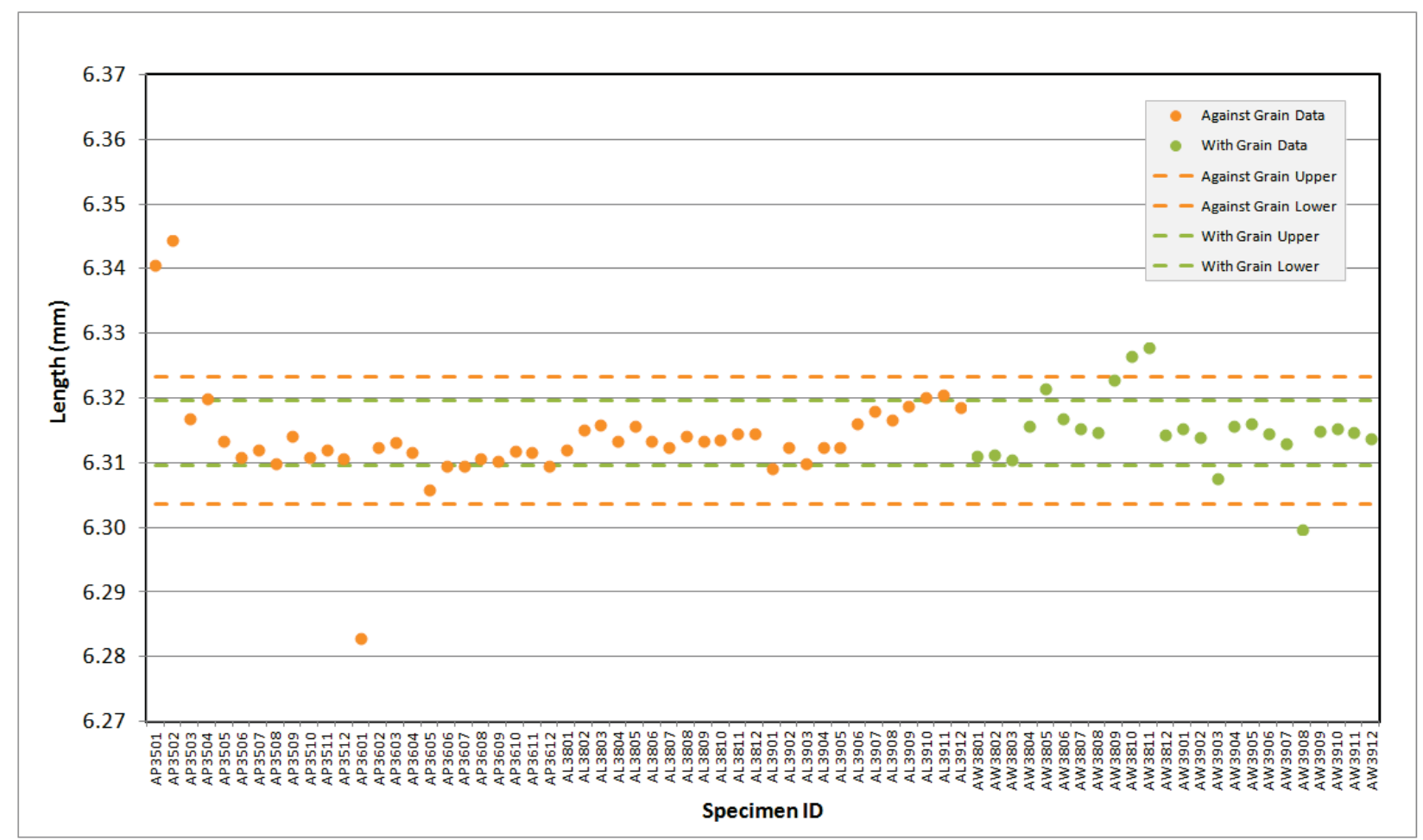

Figure A-18. NBG-17 Piggyback Pre Thermal Measurement Length.

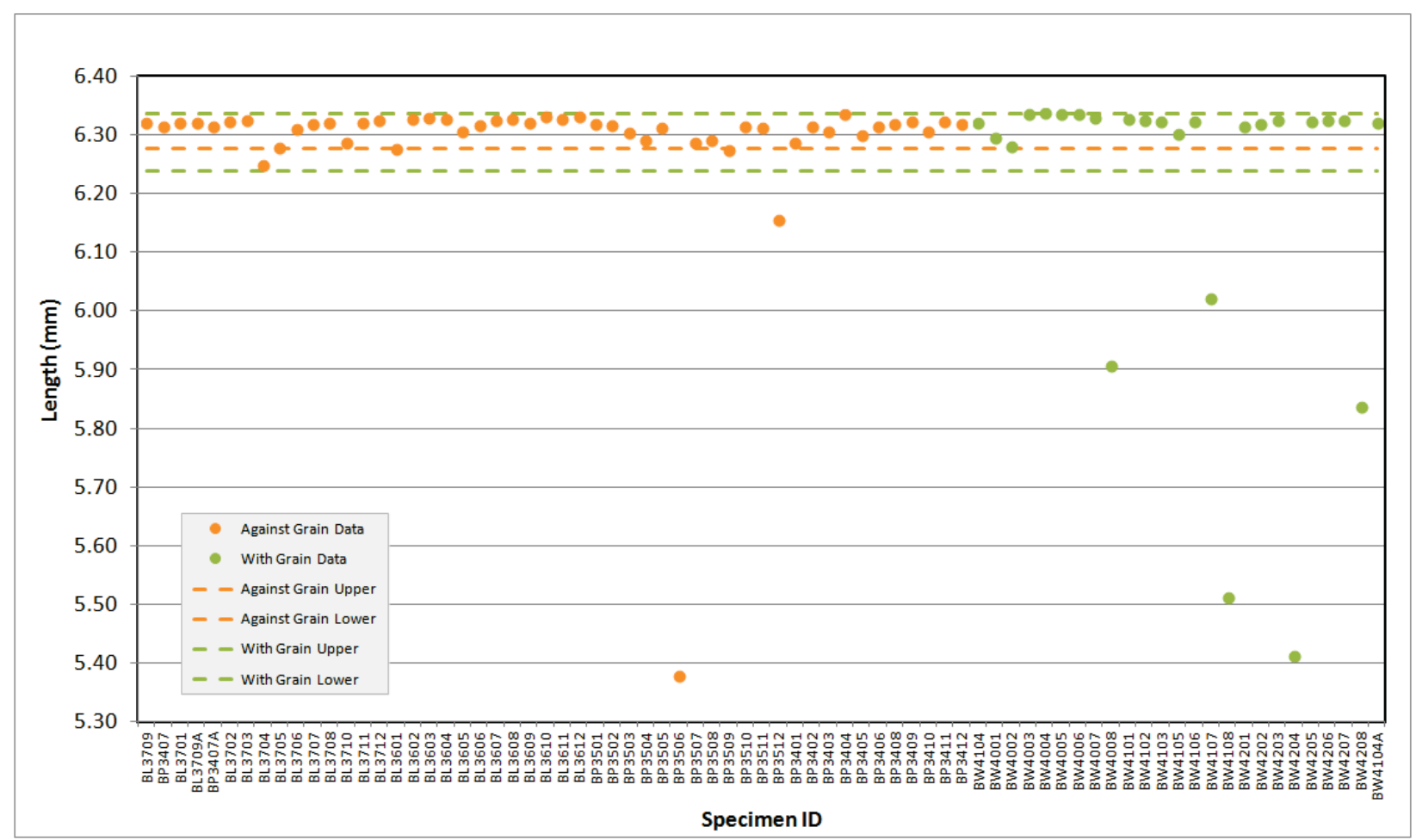

Figure A-19. NBG-18 Piggyback Pre Thermal Measurement Length. 


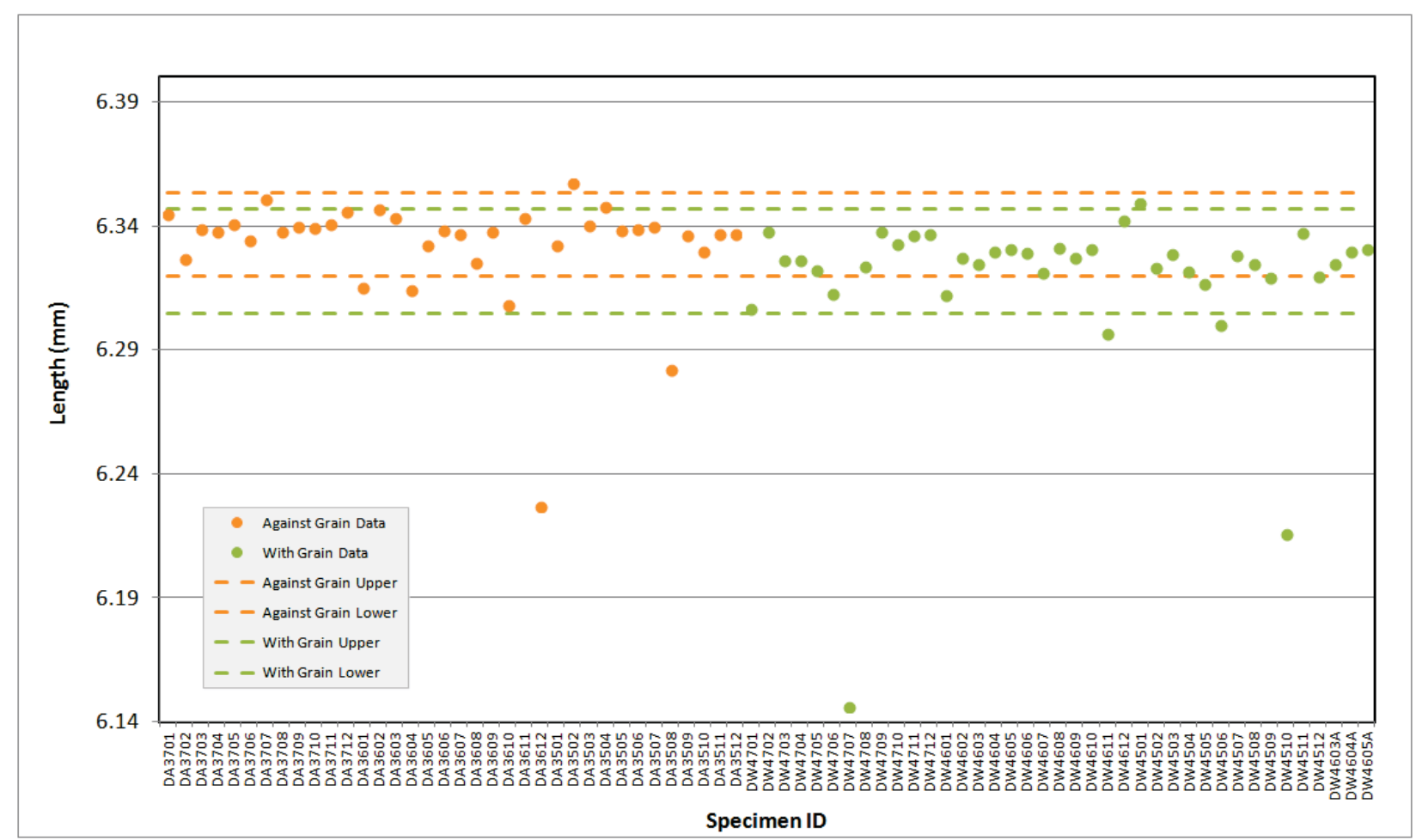

Figure A-20. PCEA Piggyback Pre Thermal Measurement Length.

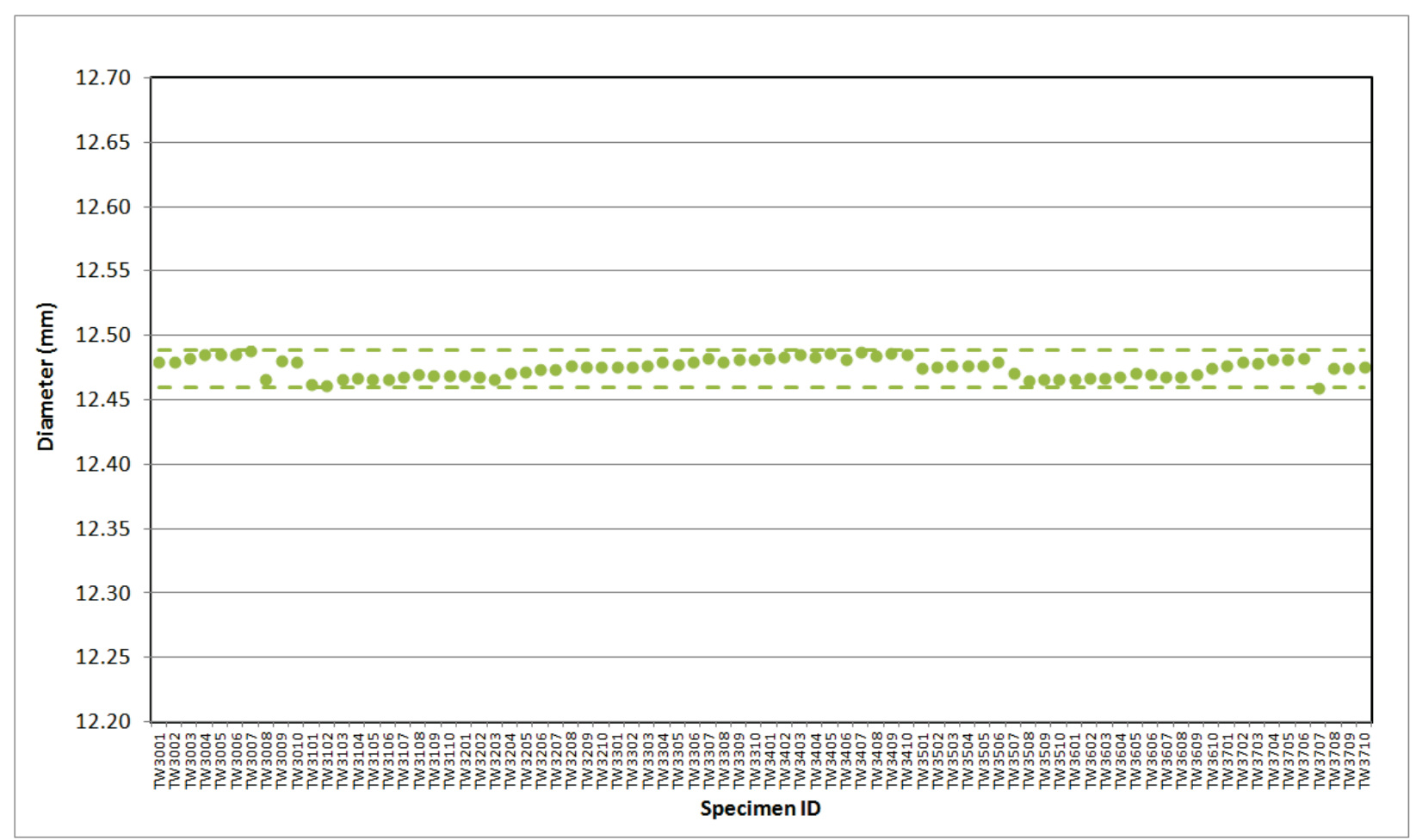

Figure A-21. 2114 Creep Pre Thermal Measurement Diameter. 


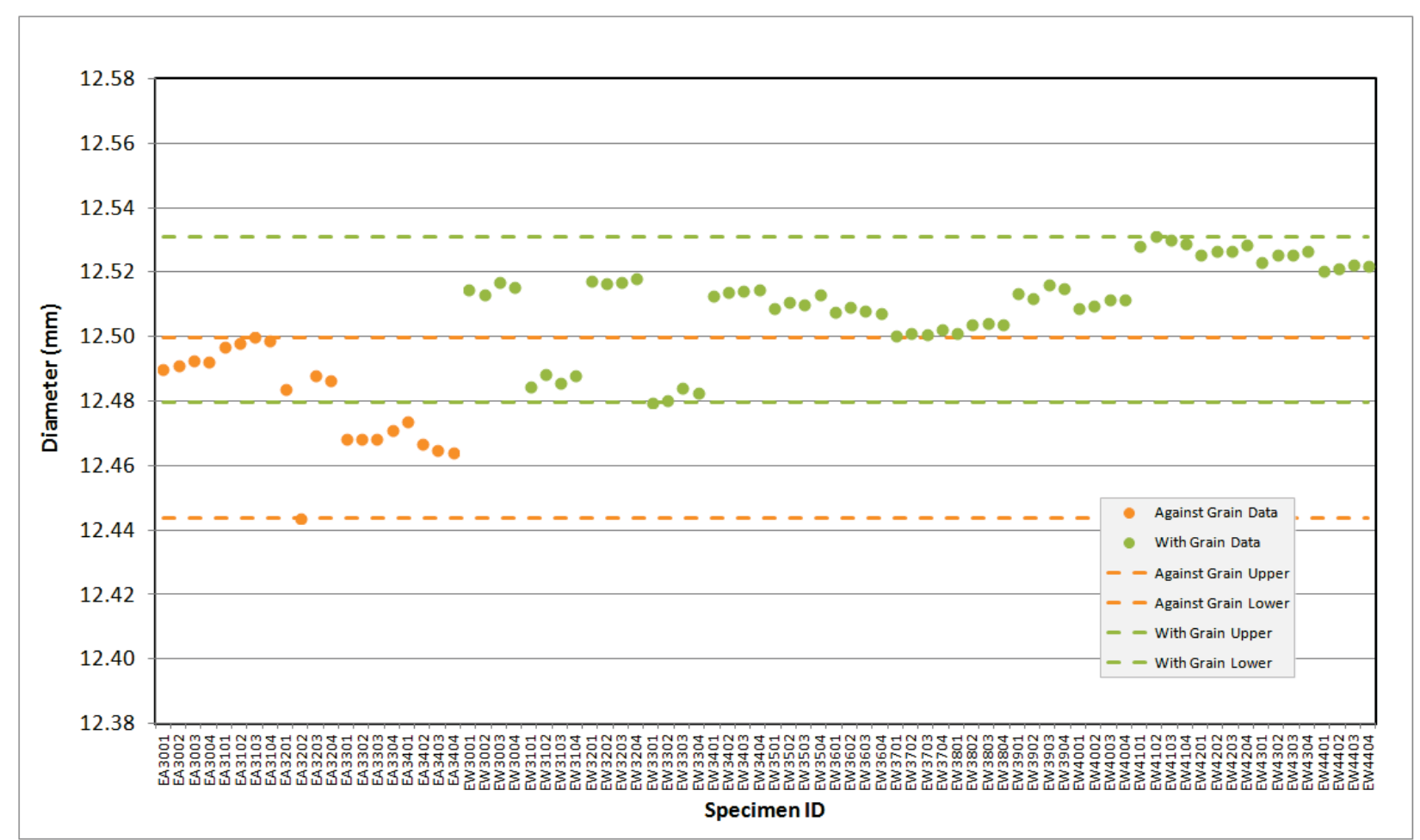

Figure A-22. IG-110 Creep Pre Thermal Measurement Diameter.

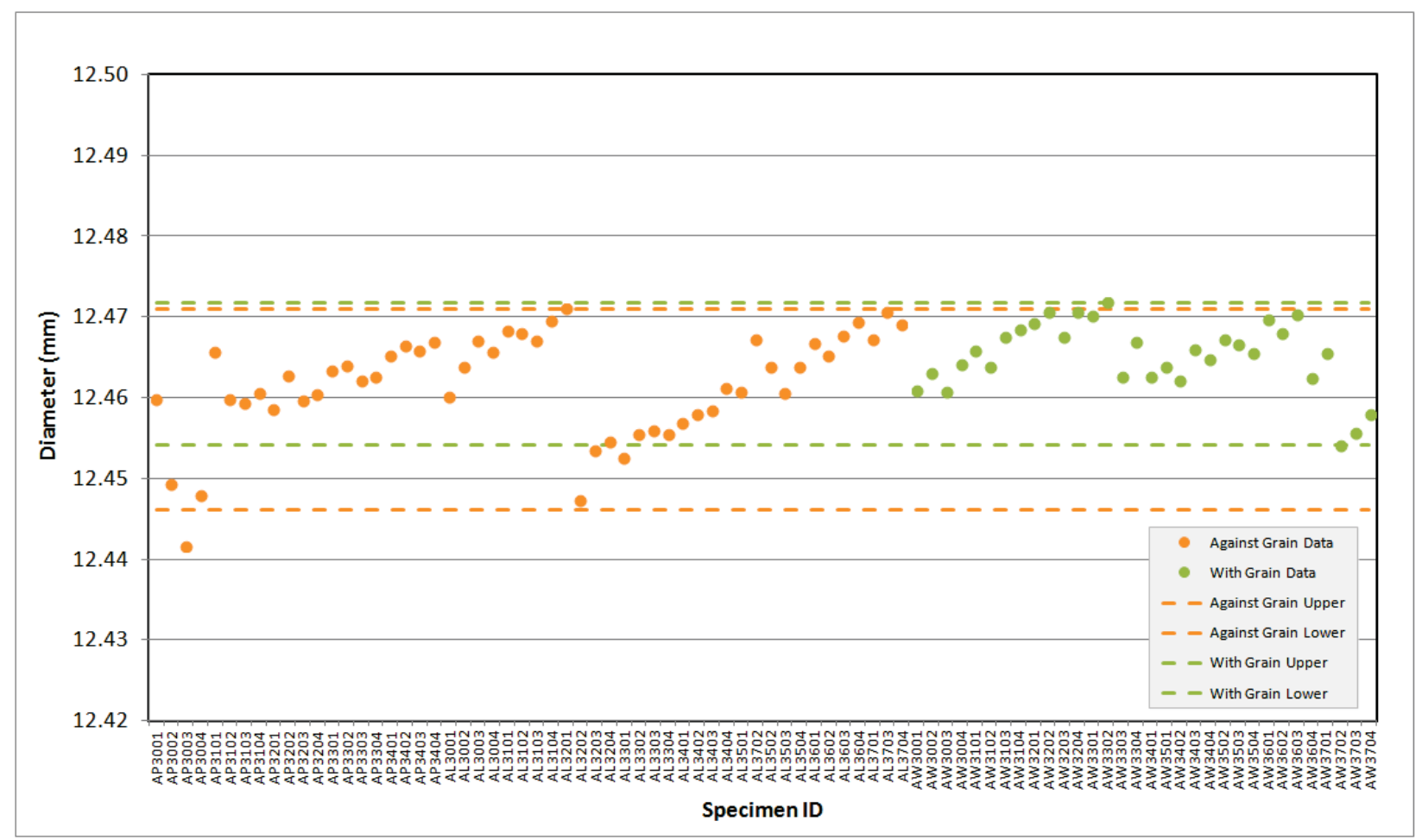

Figure A-23. NBG-17 Creep Pre Thermal Measurement Diameter. 


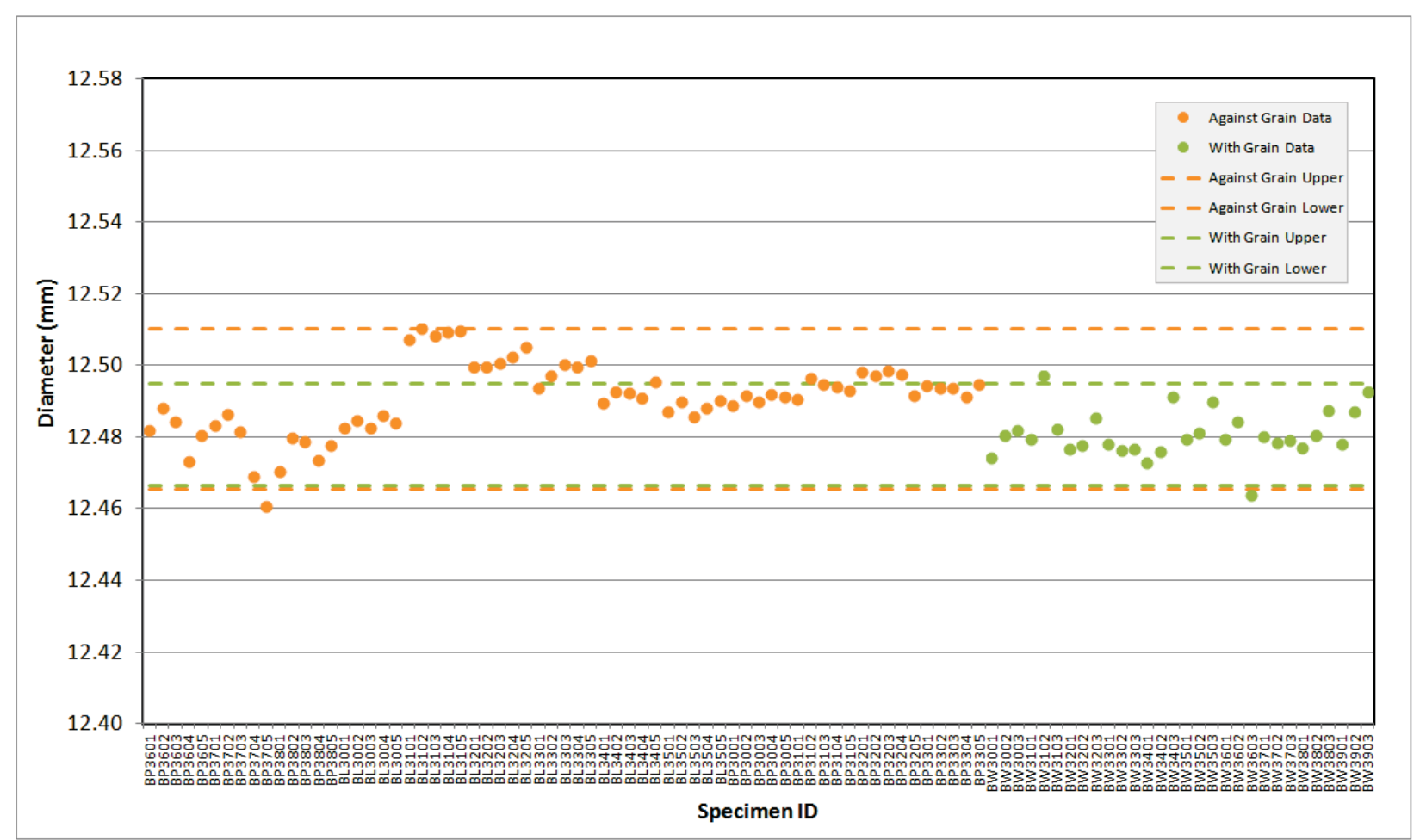

Figure A-24. NBG-18 Creep Pre Thermal Measurement Diameter.

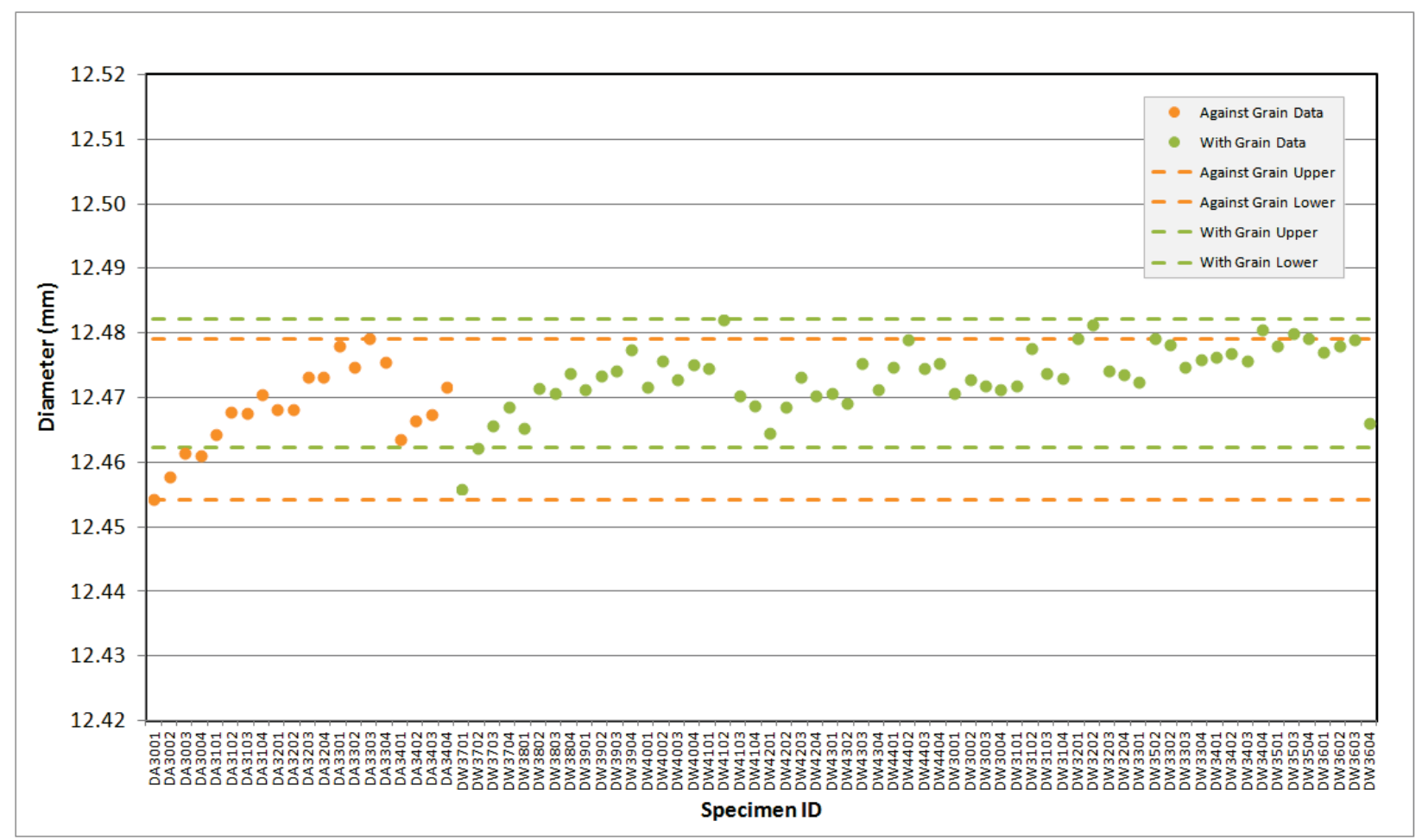

Figure A-25. PCEA Creep Pre Thermal Measurement Diameter. 


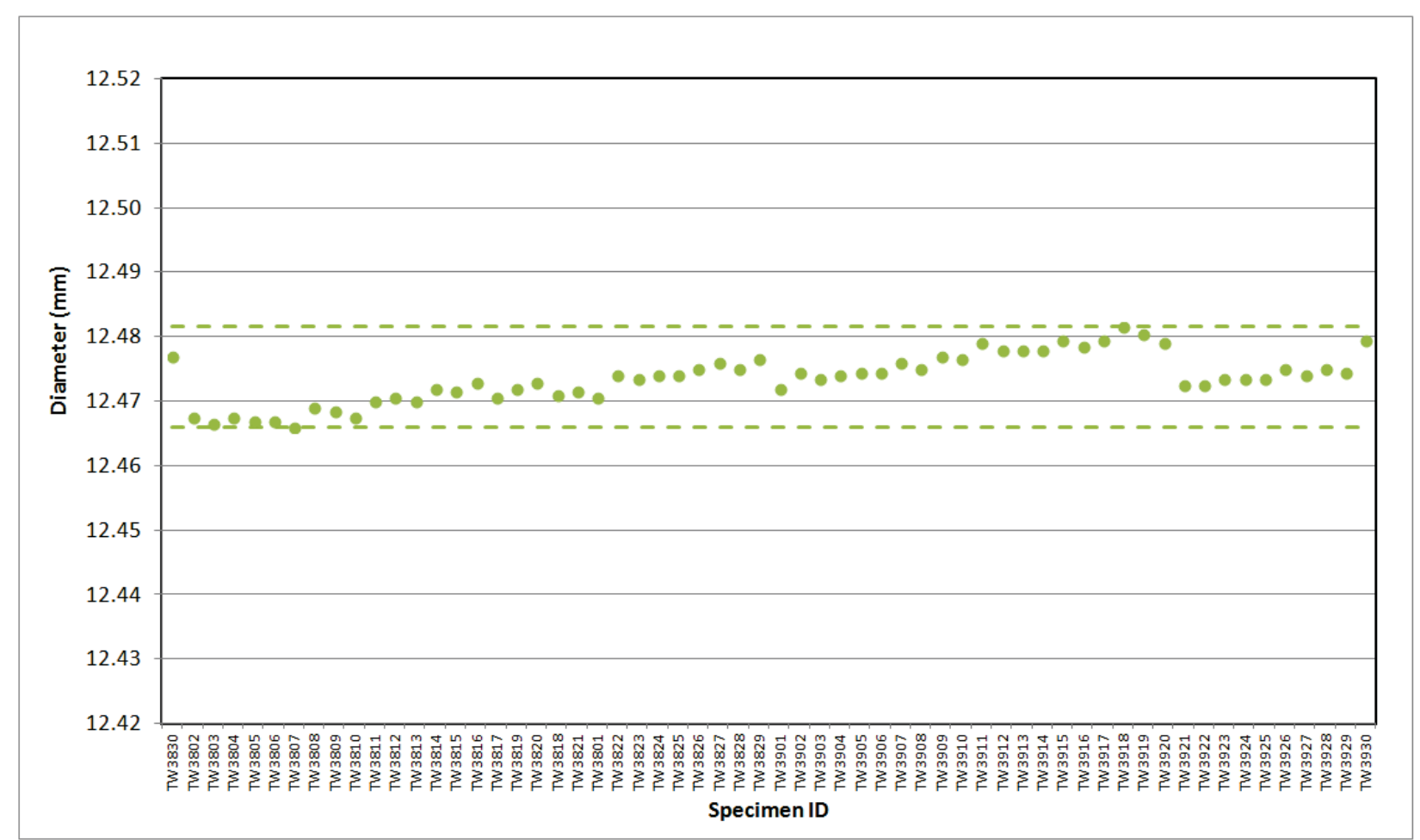

Figure A-26. 2114 Piggyback Pre Thermal Measurement Diameter.

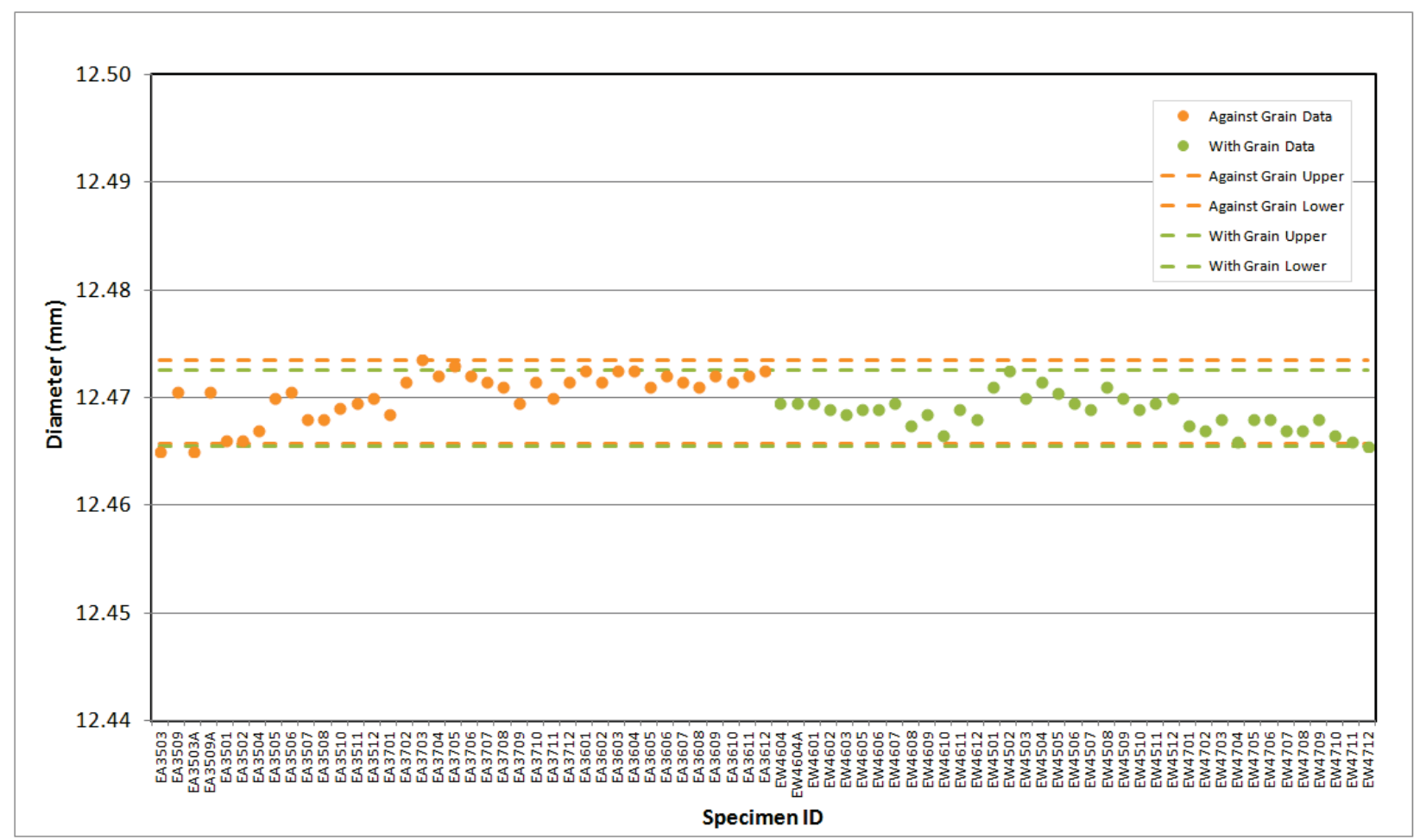

Figure A-27. IG-110 Piggyback Pre Thermal Measurement Diameter. 


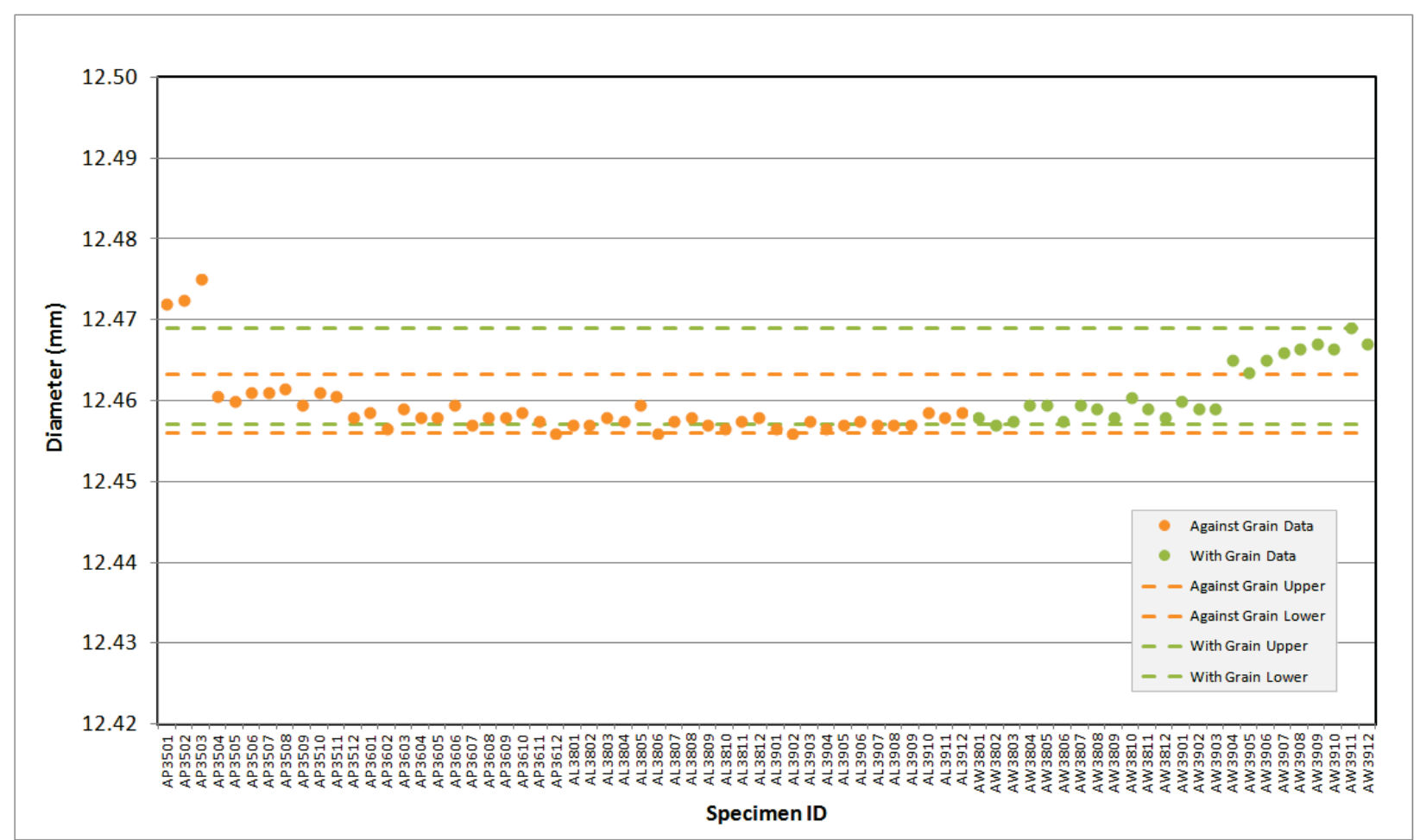

Figure A-28. NBG-17 Piggyback Pre Thermal Measurement Diameter.

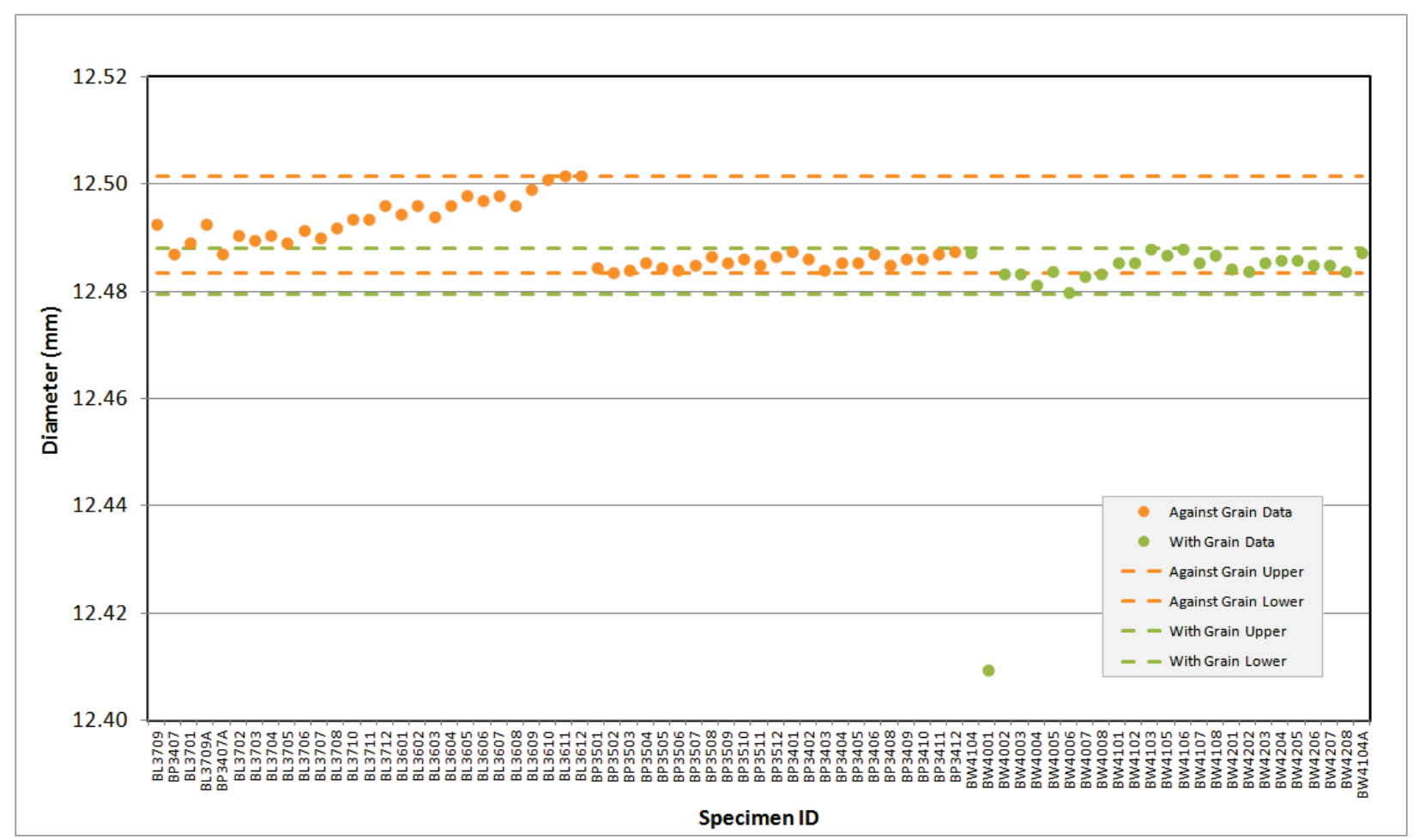

Figure A-29. NBG-18 Piggyback Pre Thermal Measurement Diameter. 


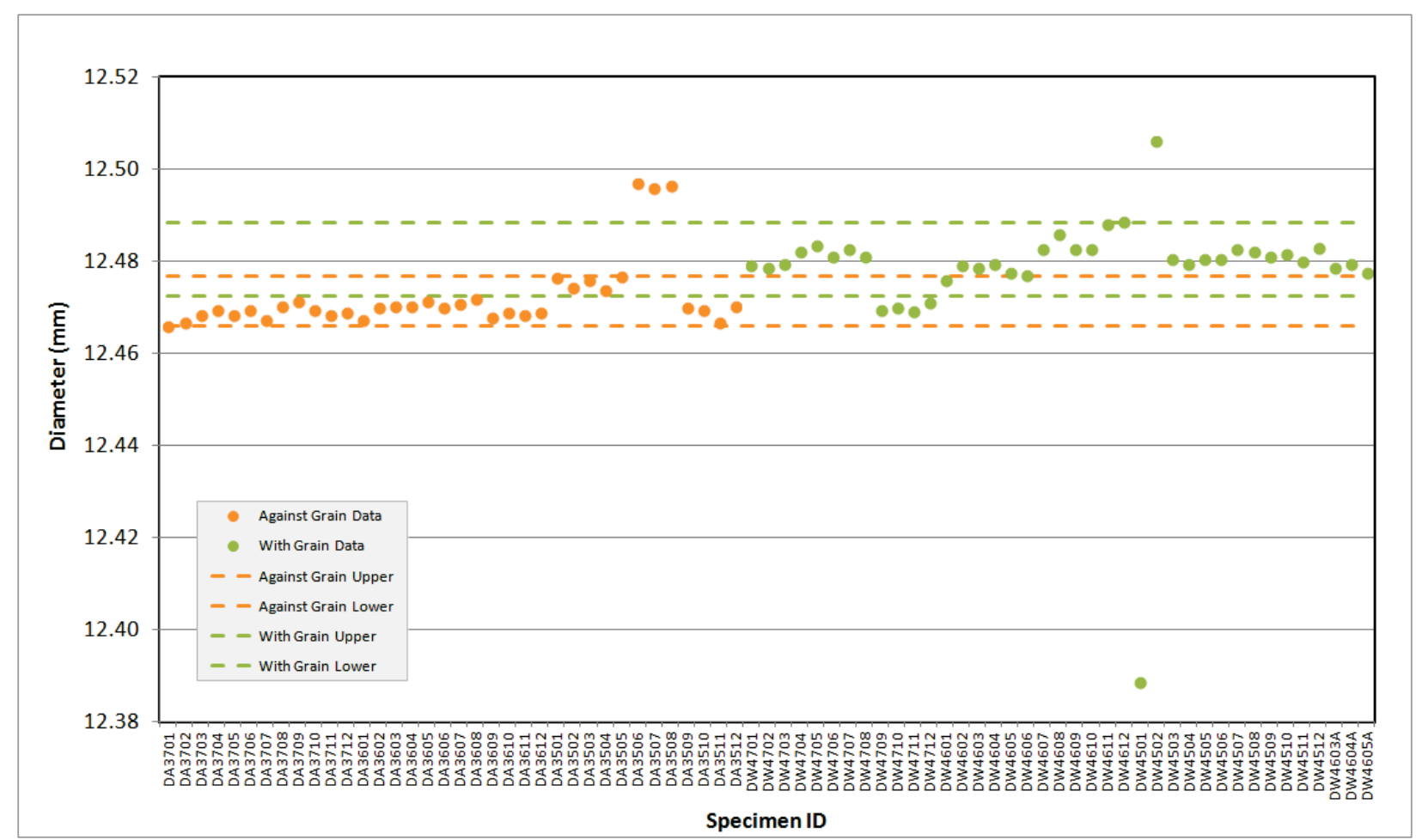

Figure A-30. PCEA Piggyback Pre Thermal Measurement Diameter.

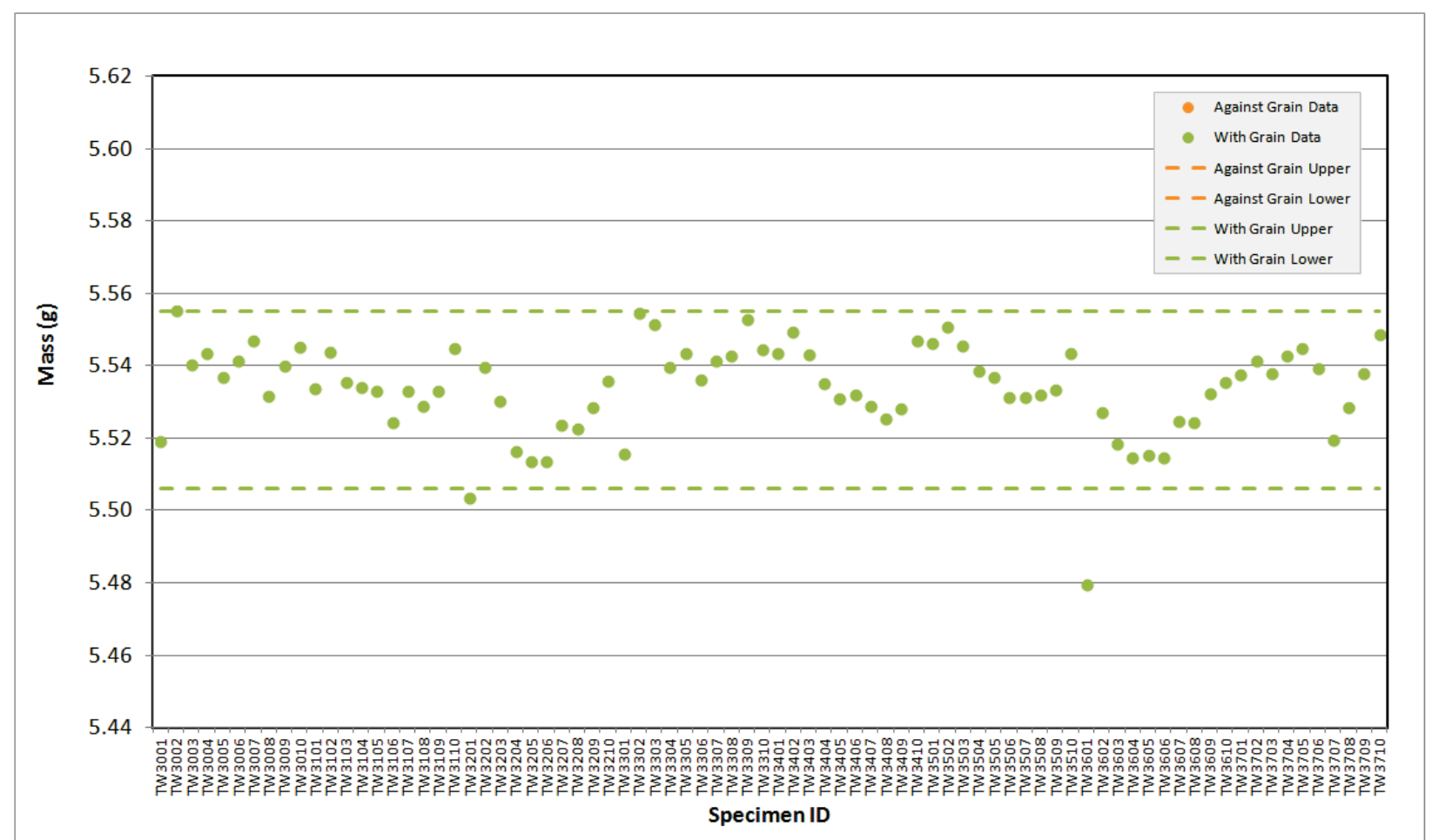

Figure A-31. 2114 Creep Pre Thermal Measurement Mass. 


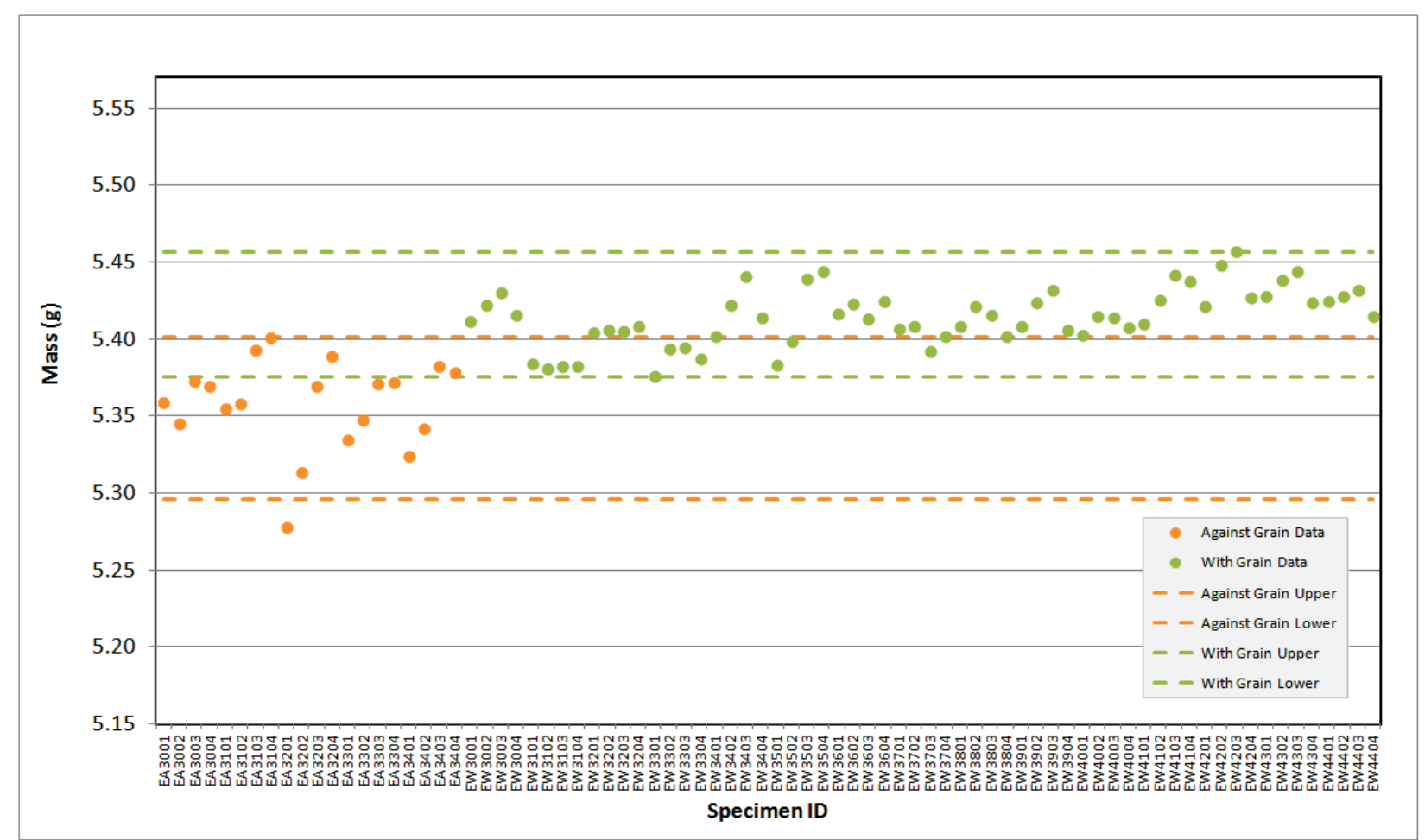

Figure A-32. IG-110 Creep Pre Thermal Measurement Mass.

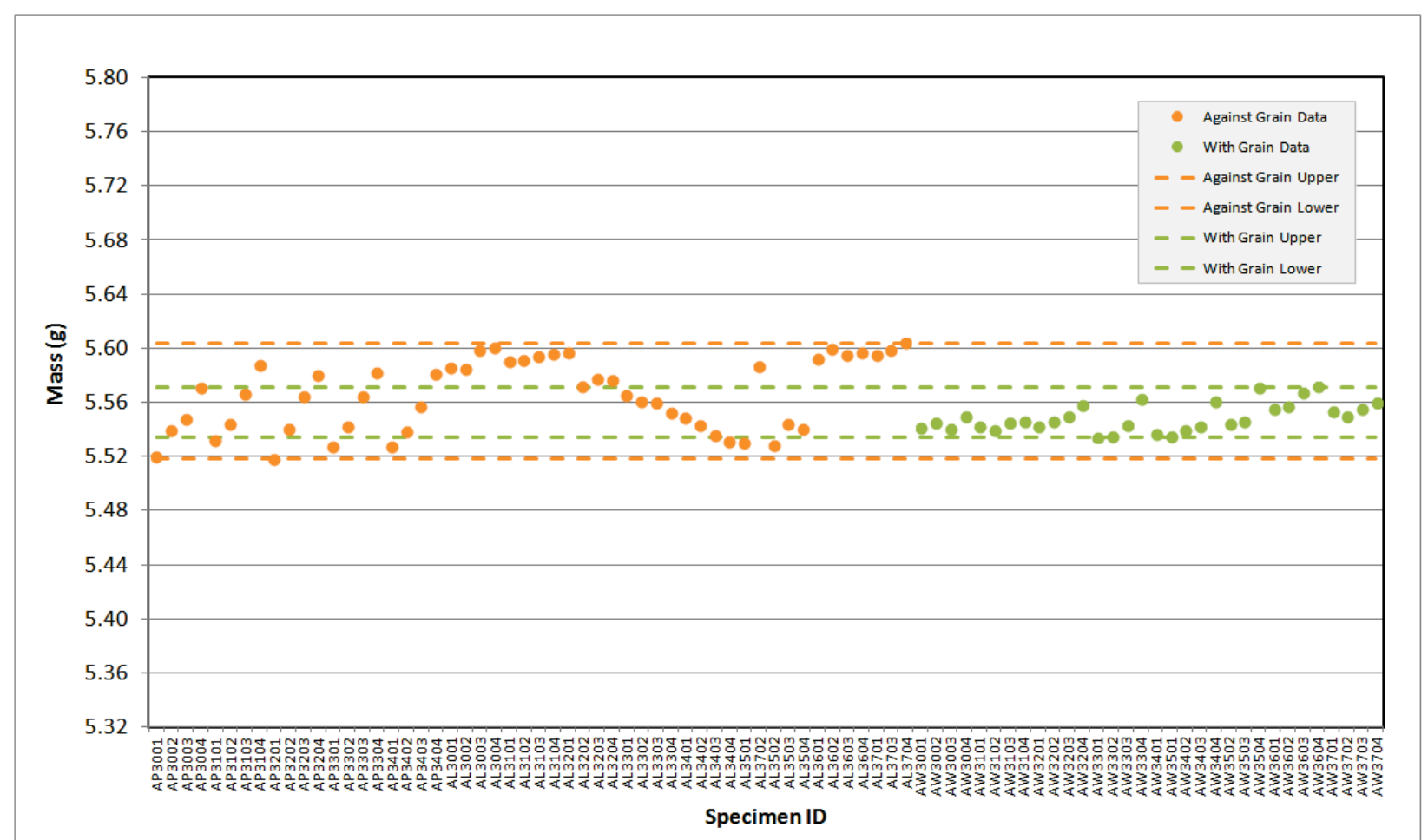

Figure A-33. NBG-17 Creep Pre Thermal Measurement Mass. 


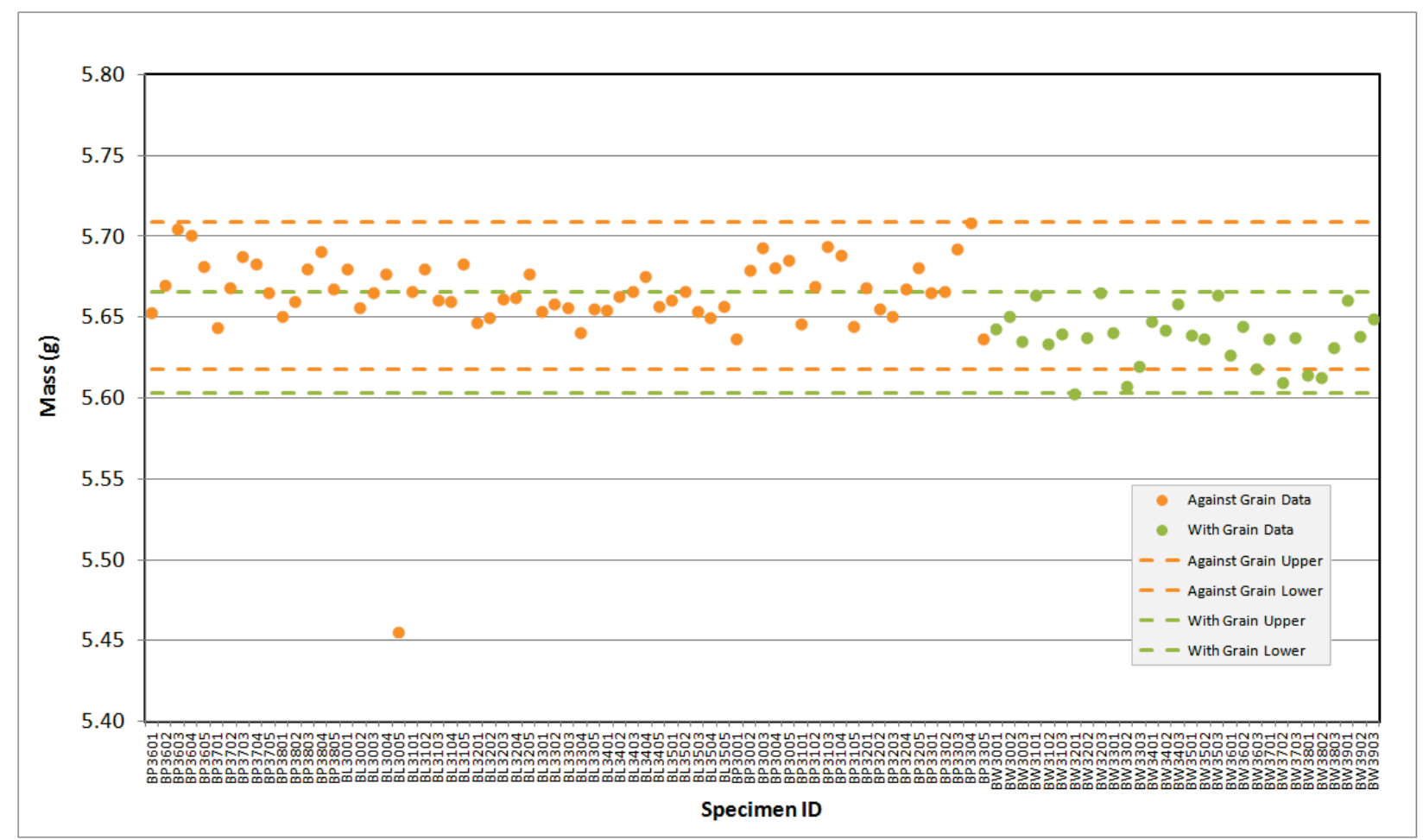

Figure A-34. NBG-18 Creep Pre Thermal Measurement Mass.

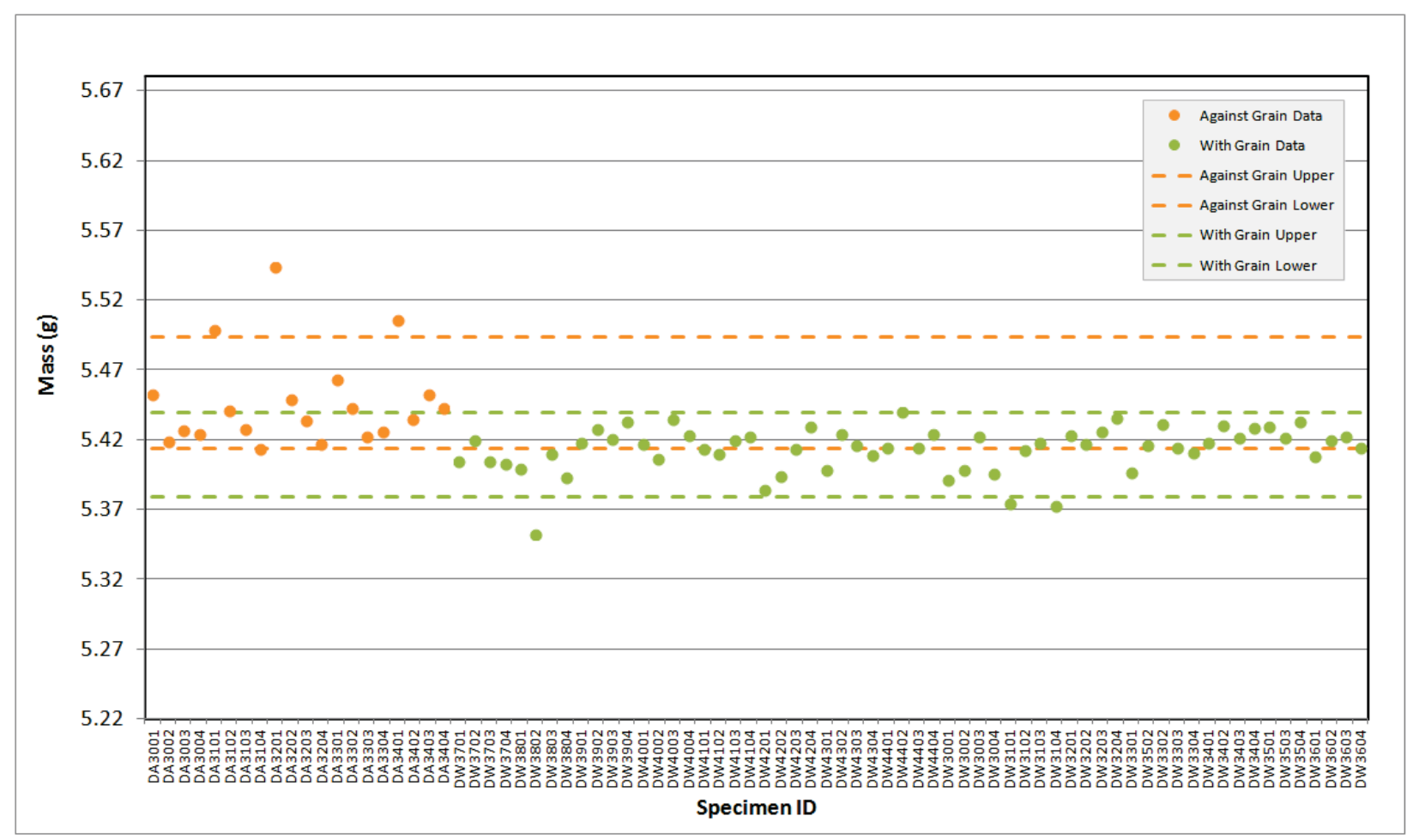

Figure A-35. PCEA Creep Pre Thermal Measurement Mass. 


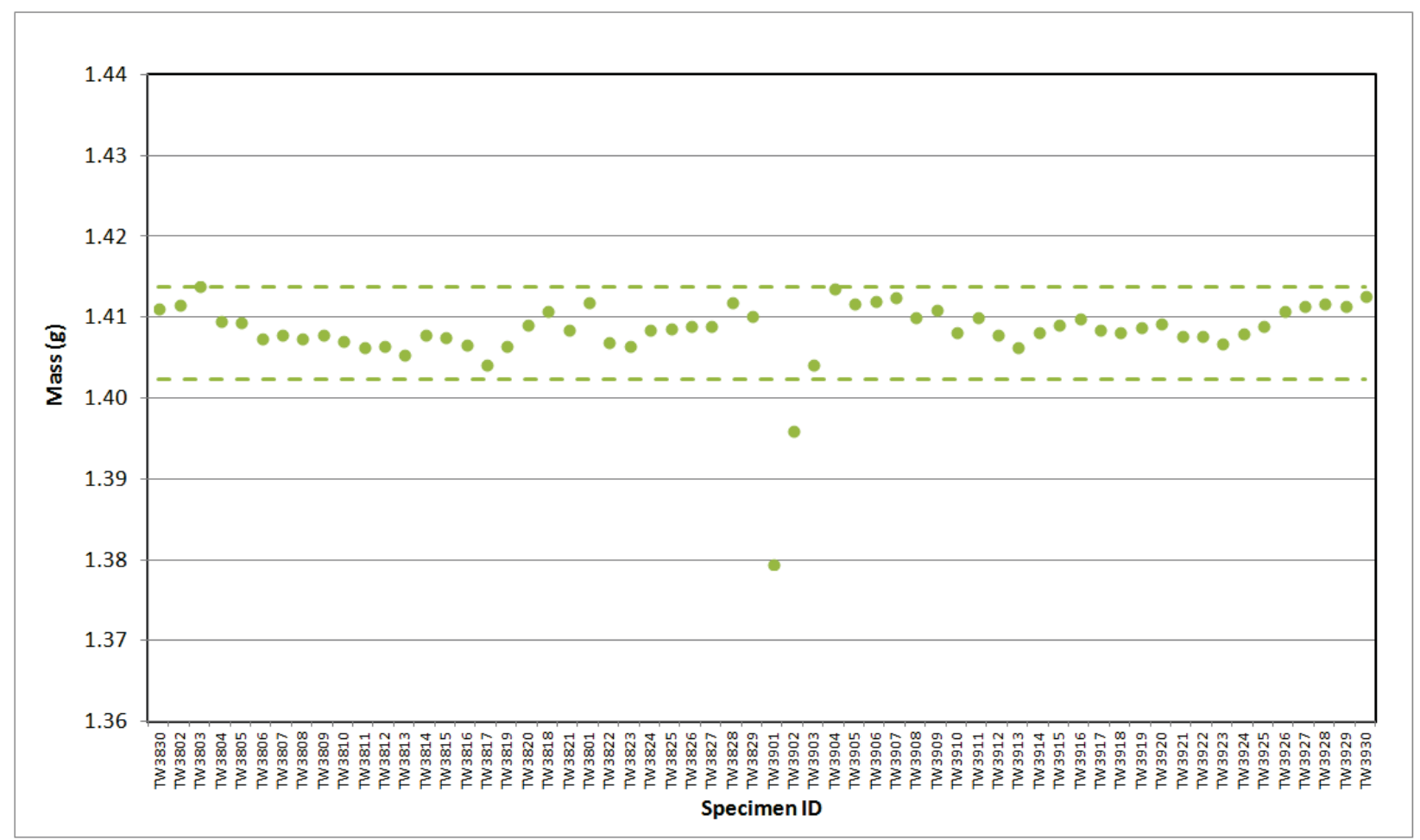

Figure A-36. 2114 Piggyback Pre Thermal Measurement Mass.

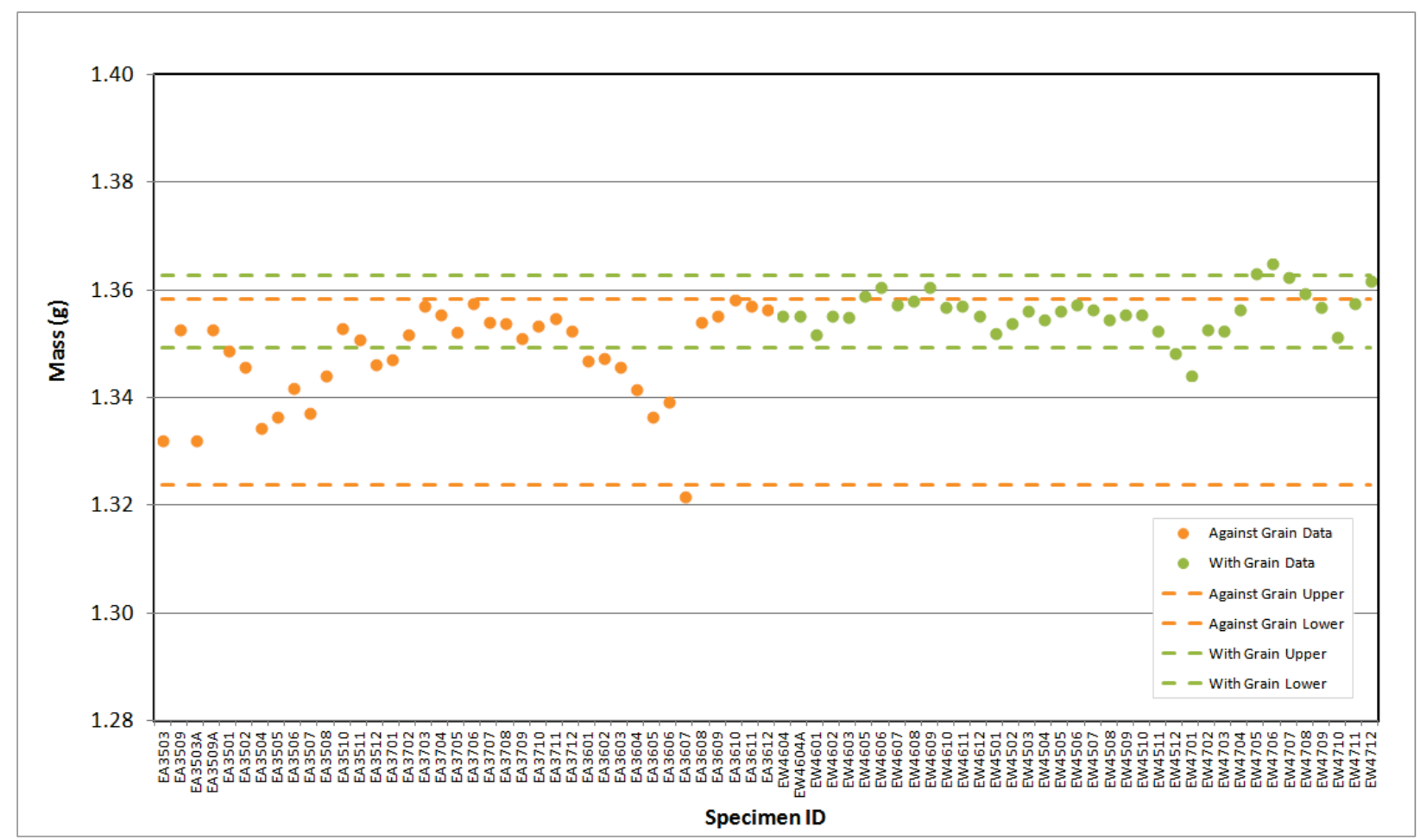

Figure A-37. IG-110 Piggyback Pre Thermal Measurement Mass. 


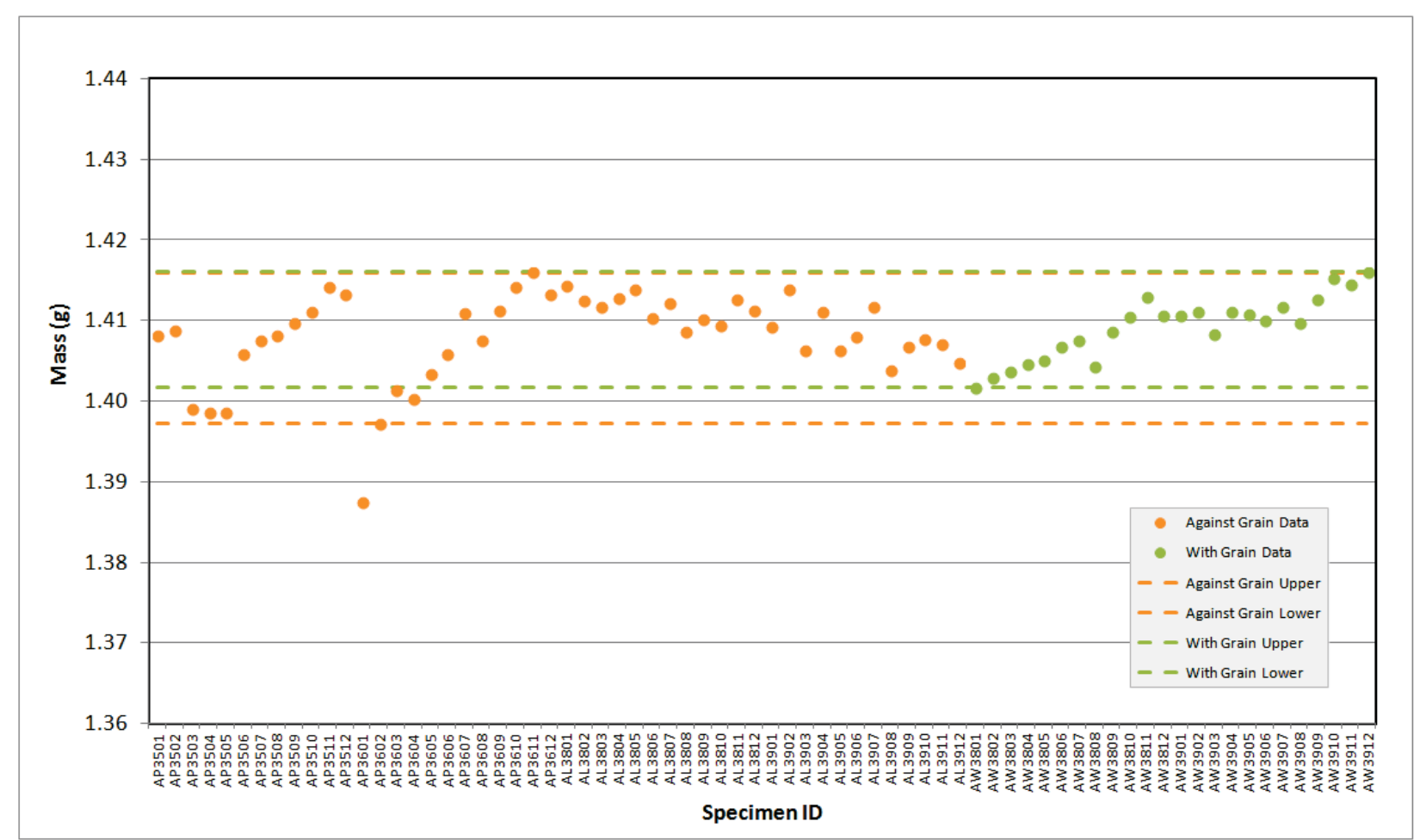

Figure A-38. NBG-17 Piggyback Pre Thermal Measurement Mass.

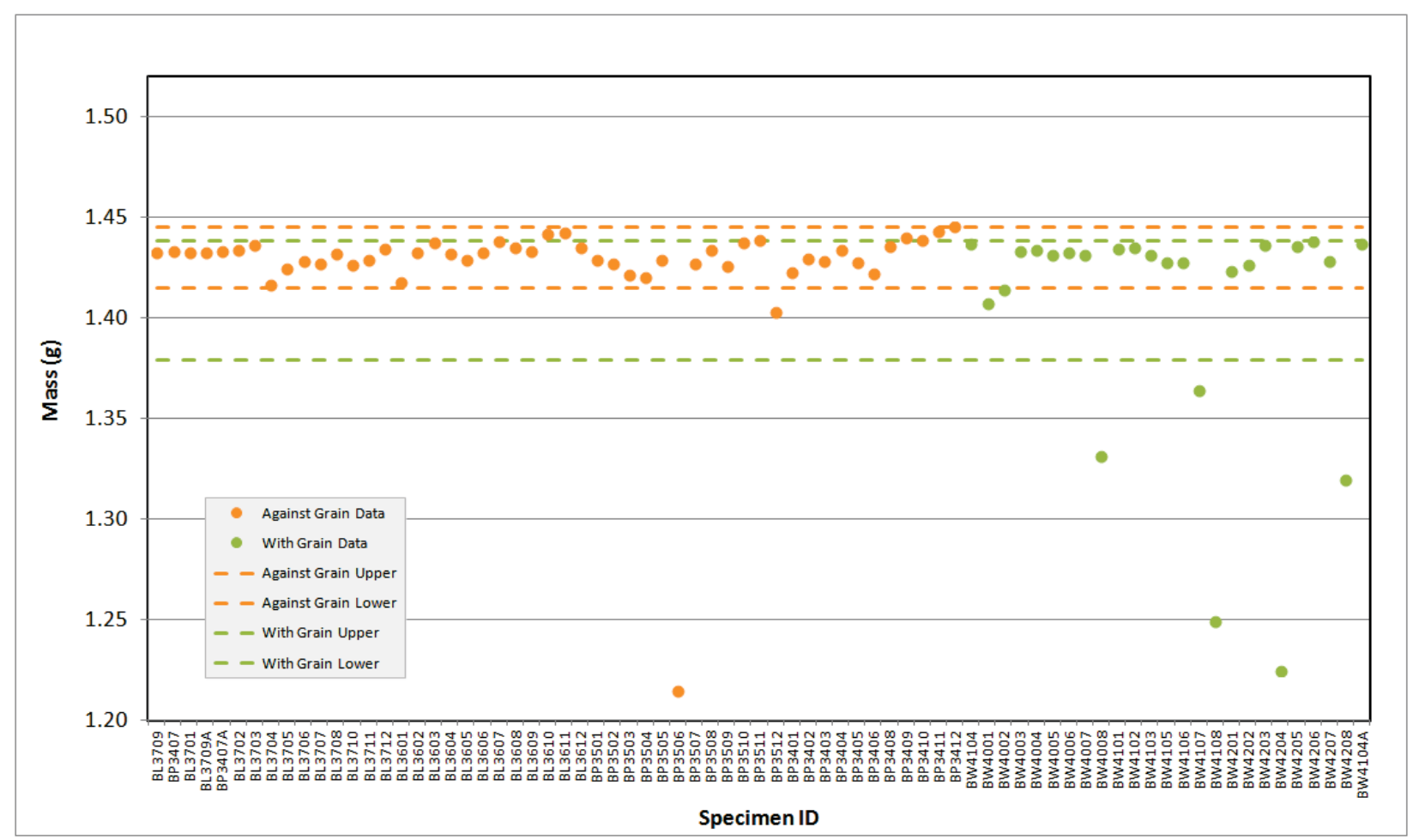

Figure A-39. NBG-18 Piggyback Pre Thermal Measurement Mass. 


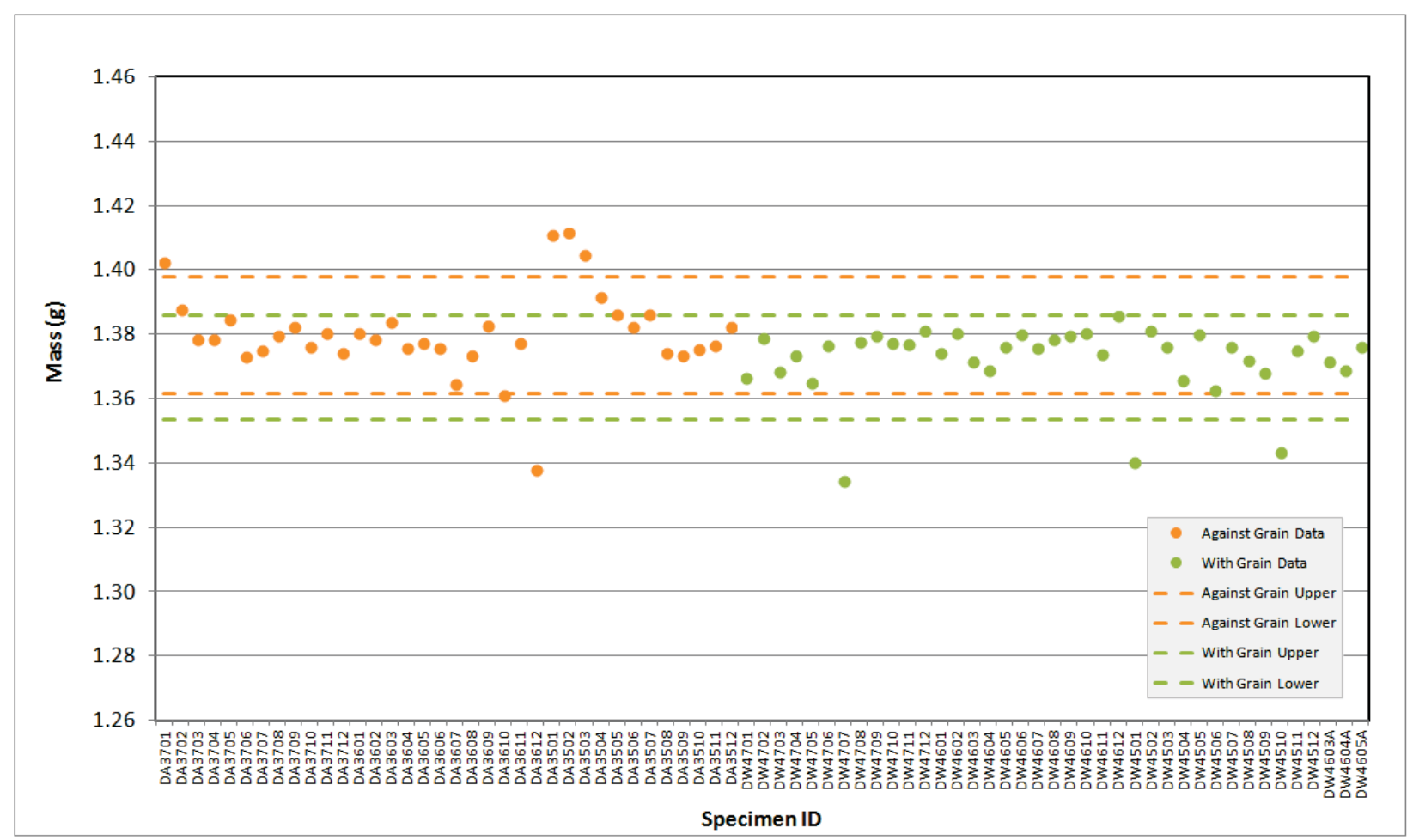

Figure A-40. PCEA Piggyback Pre Thermal Measurement Mass.

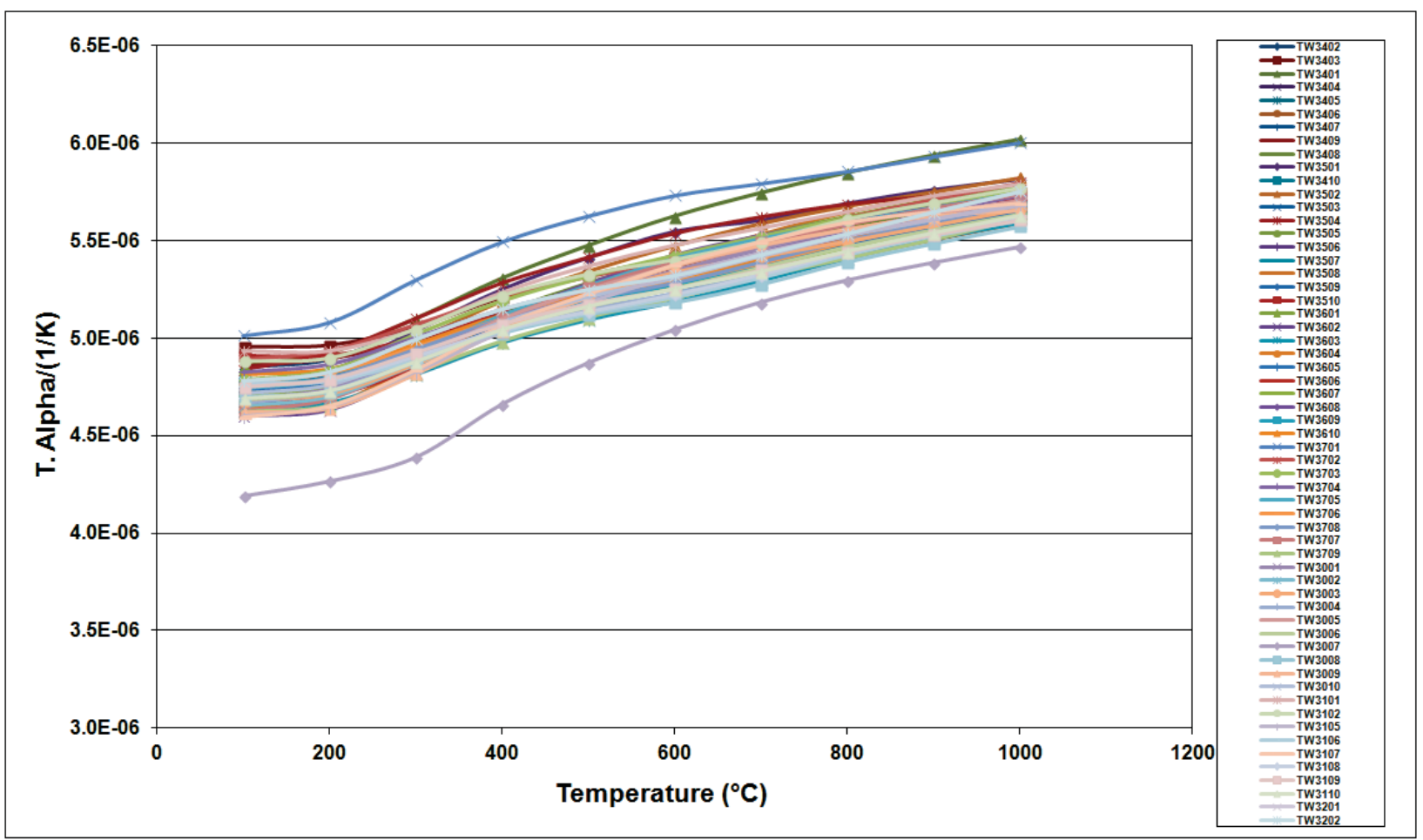

Figure A-41. 2114 Creep Coefficient of Thermal Expansion. 


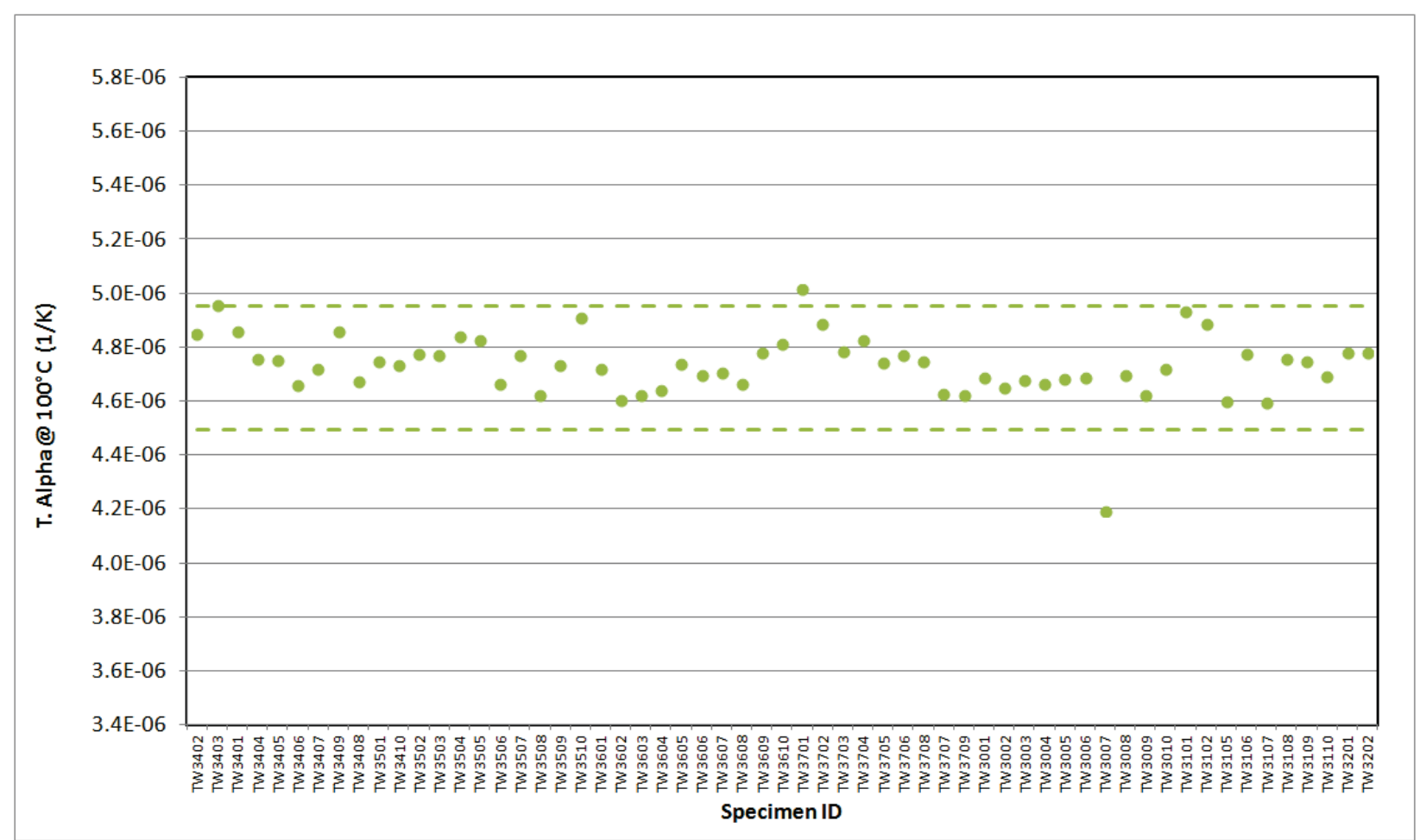

Figure A-42.2114 Creep Coefficient of Thermal Expansion @ $100^{\circ} \mathrm{C}$.

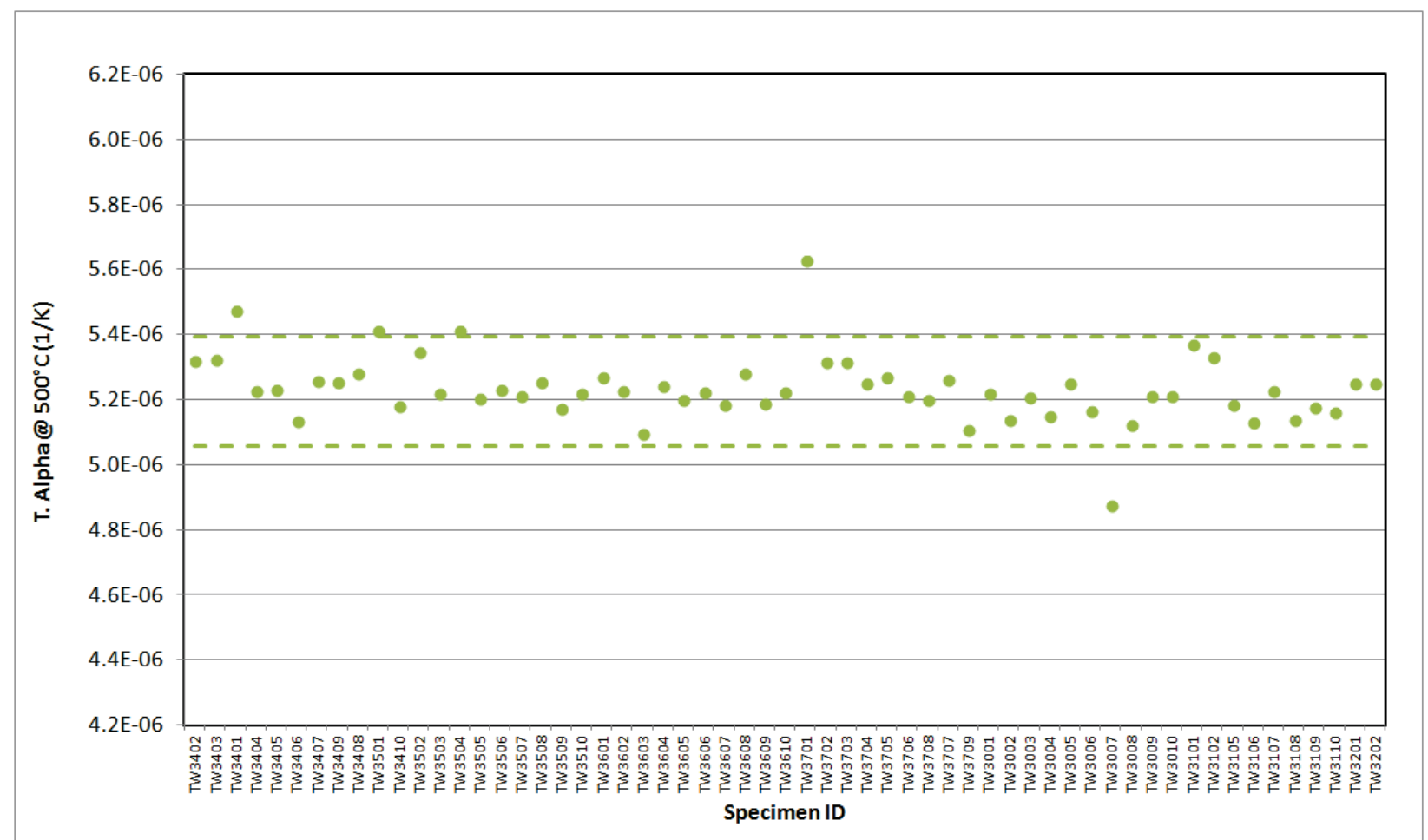

Figure A-43.2114 Creep Coefficient of Thermal Expansion @ $500^{\circ} \mathrm{C}$. 


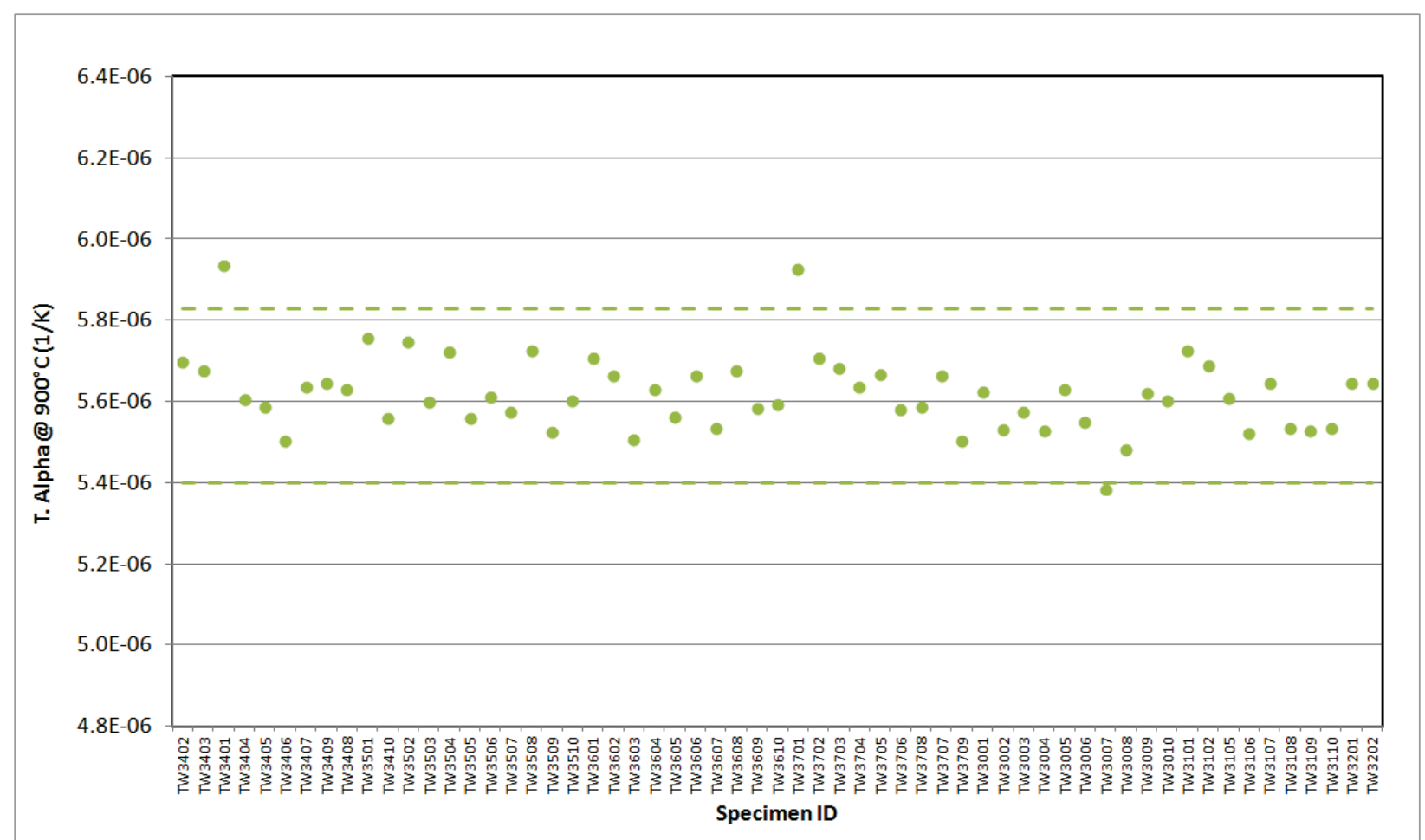

Figure A-44. 2114 Creep Coefficient of Thermal Expansion @ $900^{\circ} \mathrm{C}$.

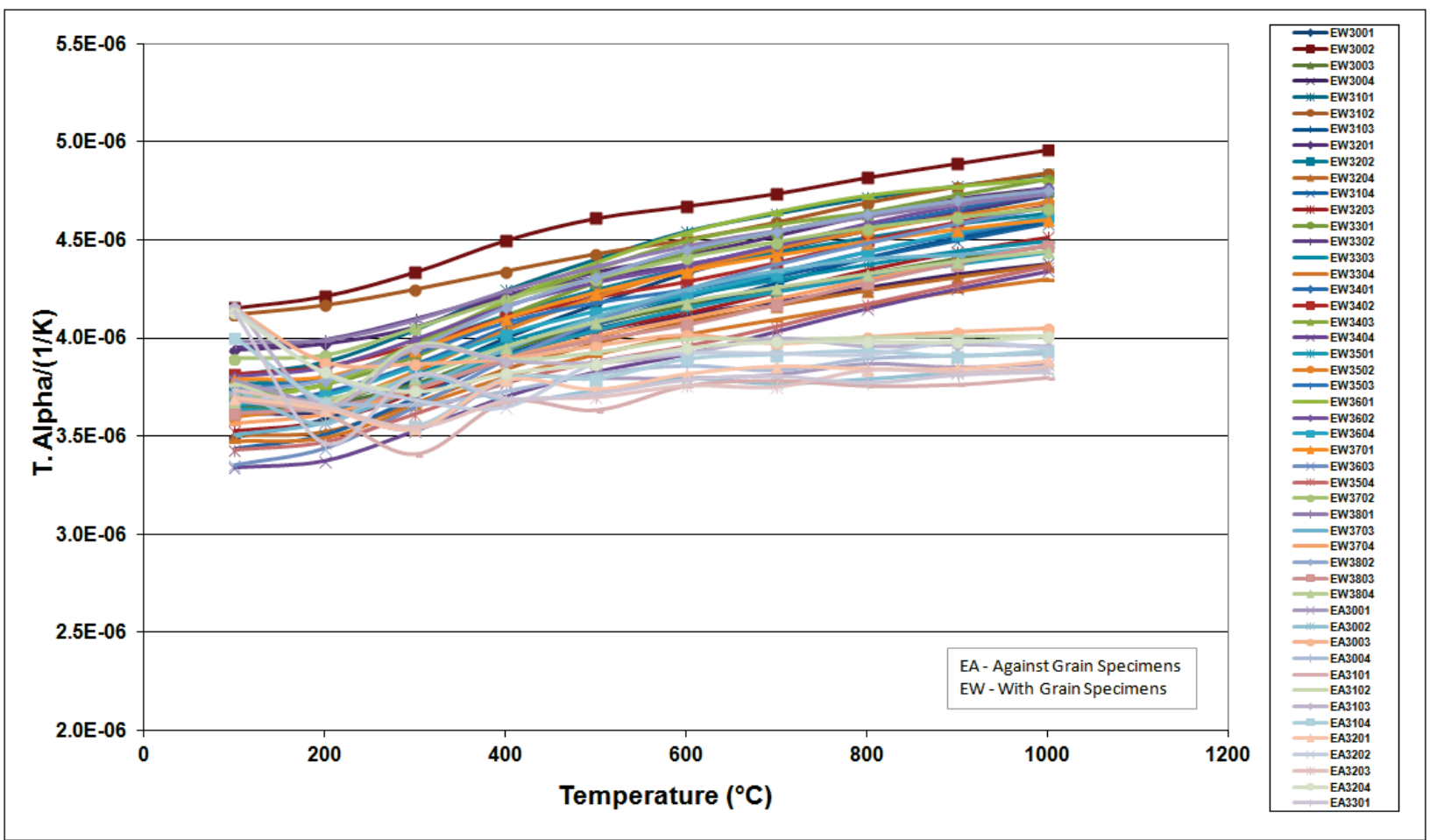

Figure A-45. IG-110 Creep Coefficient of Thermal Expansion. 


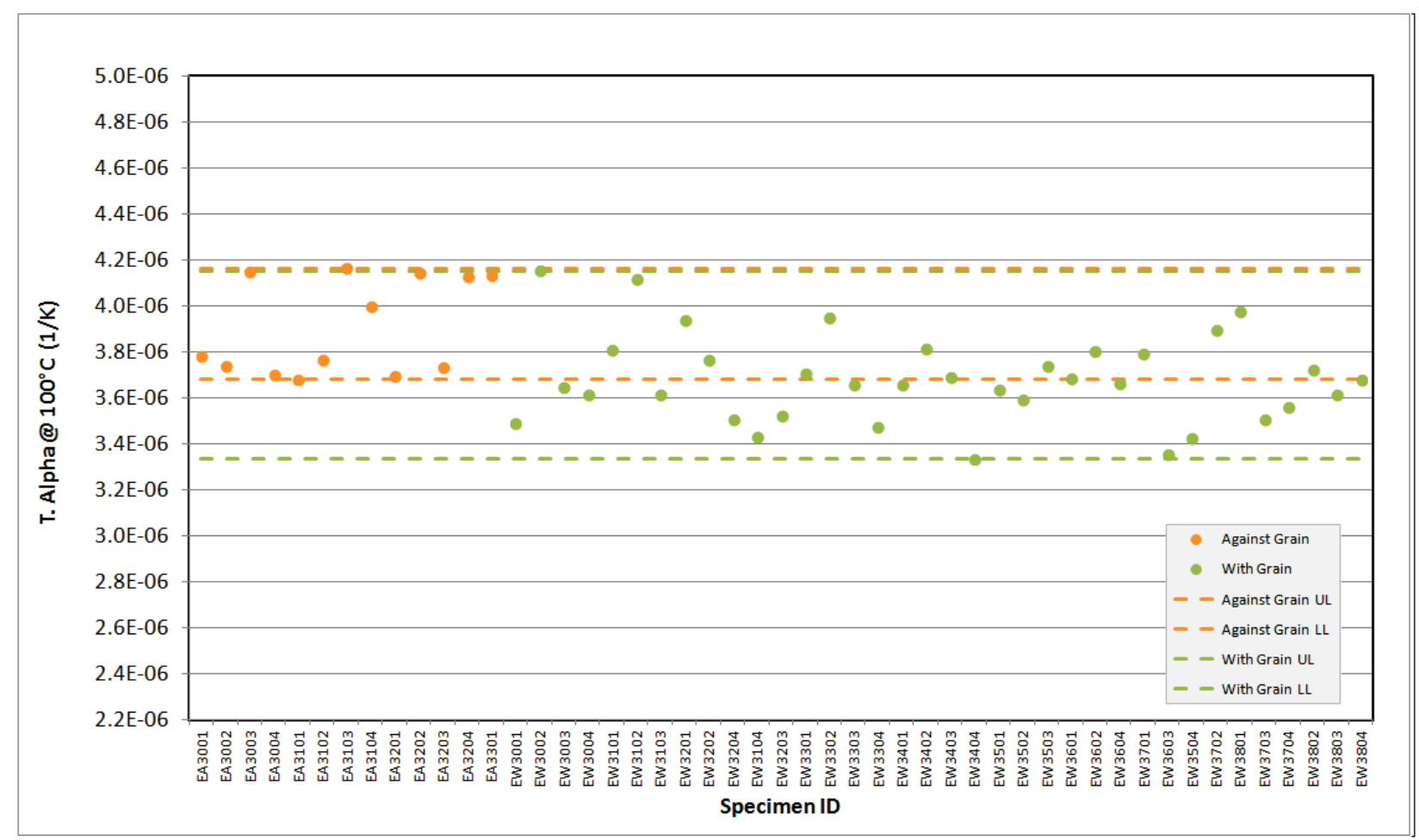

Figure A-46. IG-110 Creep Coefficient of Thermal Expansion @ $100^{\circ} \mathrm{C}$.

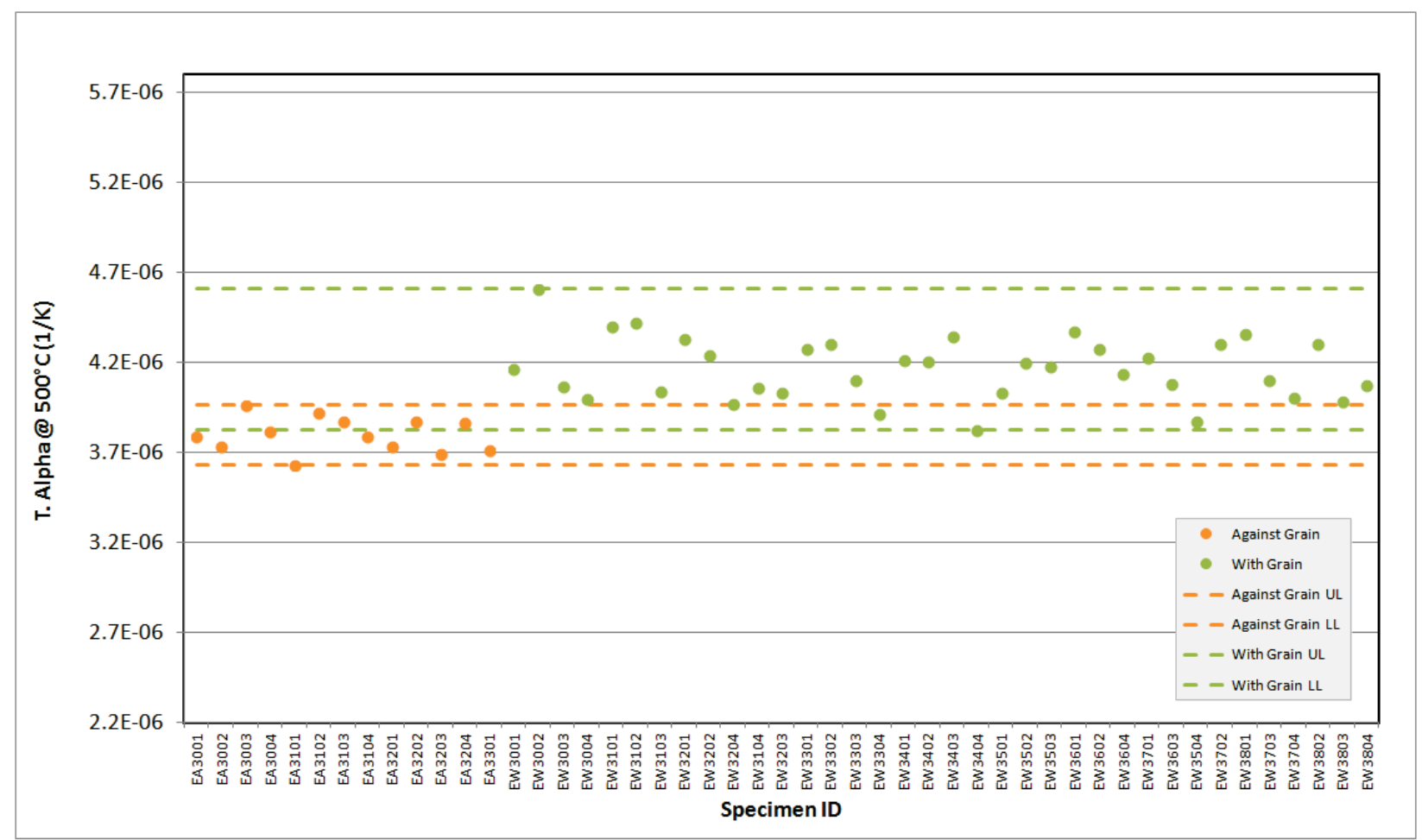

Figure A-47. IG-110 Creep Coefficient of Thermal Expansion @ $500^{\circ} \mathrm{C}$. 


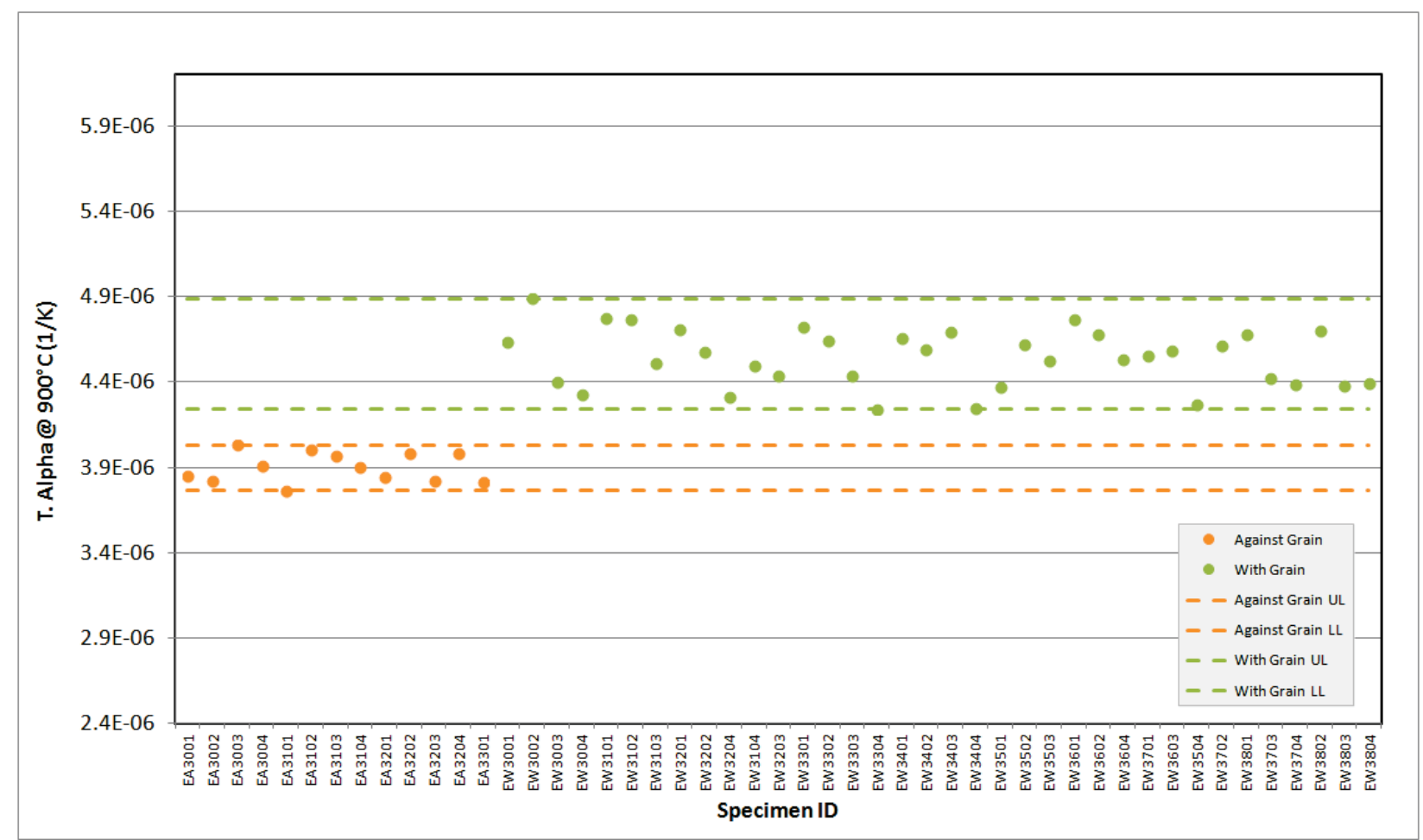

Figure A-48. IG-110 Creep Coefficient of Thermal Expansion @ $900^{\circ} \mathrm{C}$.

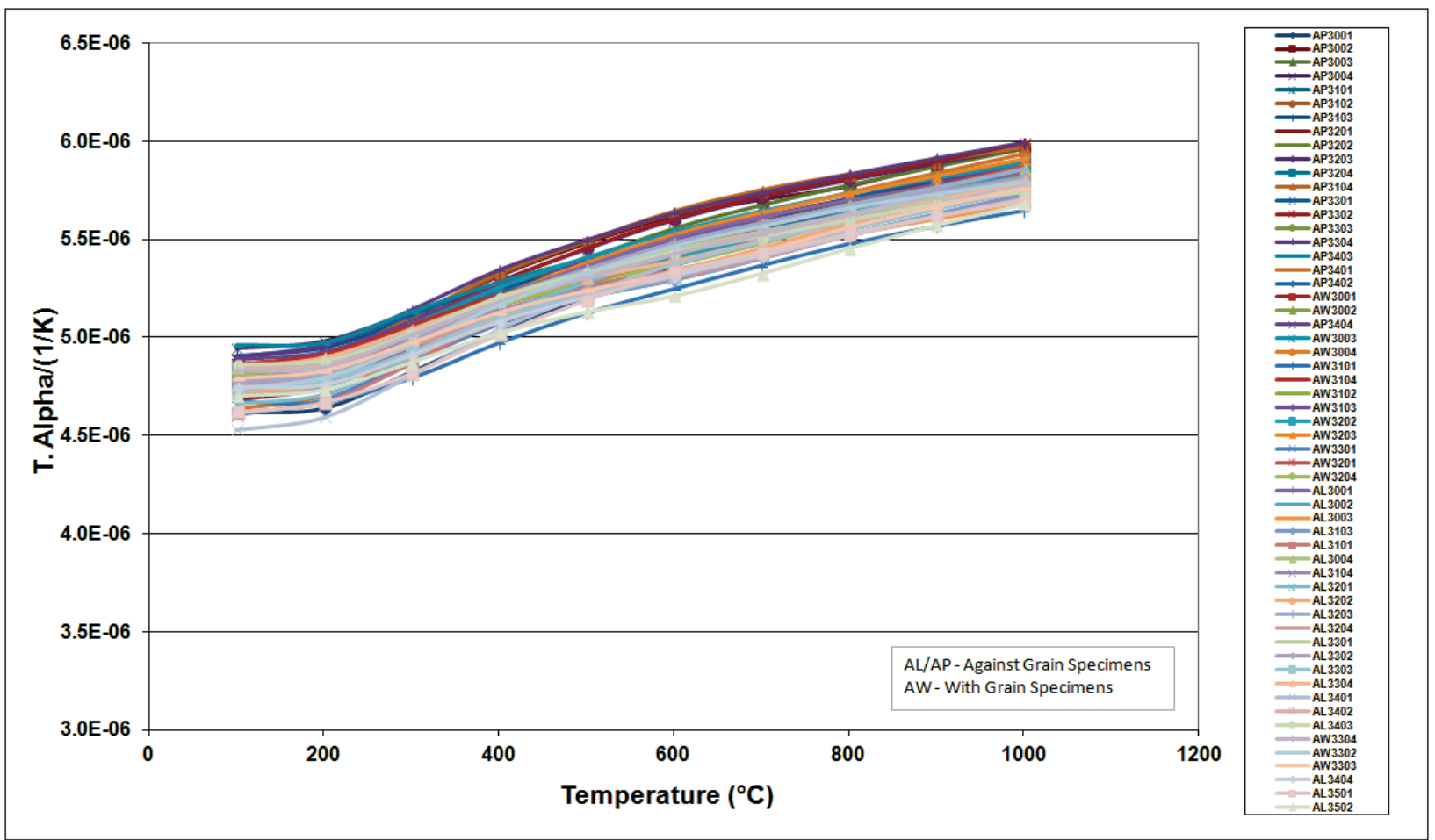

Figure A-49. NBG-17 Creep Coefficient of Thermal Expansion. 


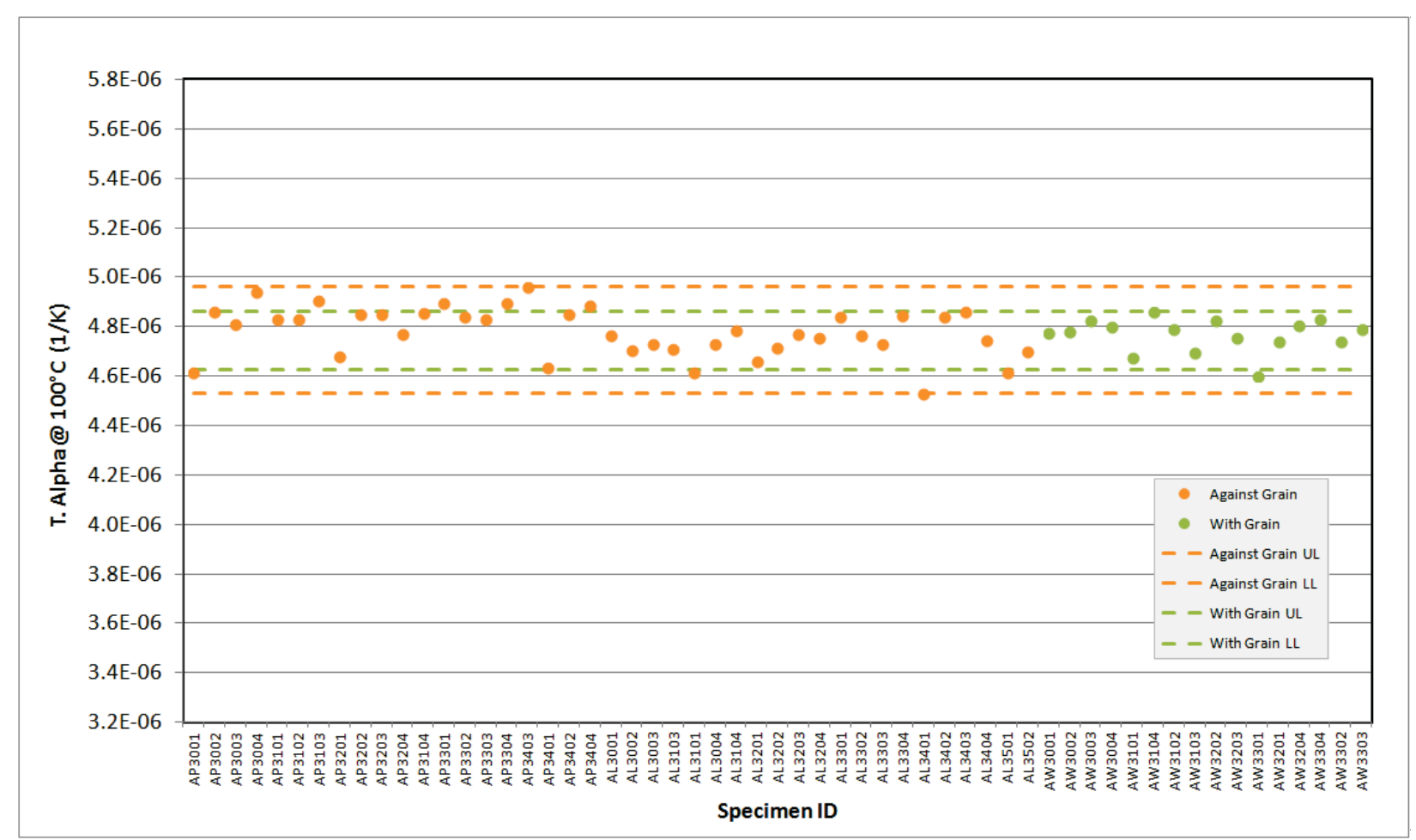

Figure A-50. NBG-17 Creep Coefficient of Thermal Expansion @ $100^{\circ} \mathrm{C}$.

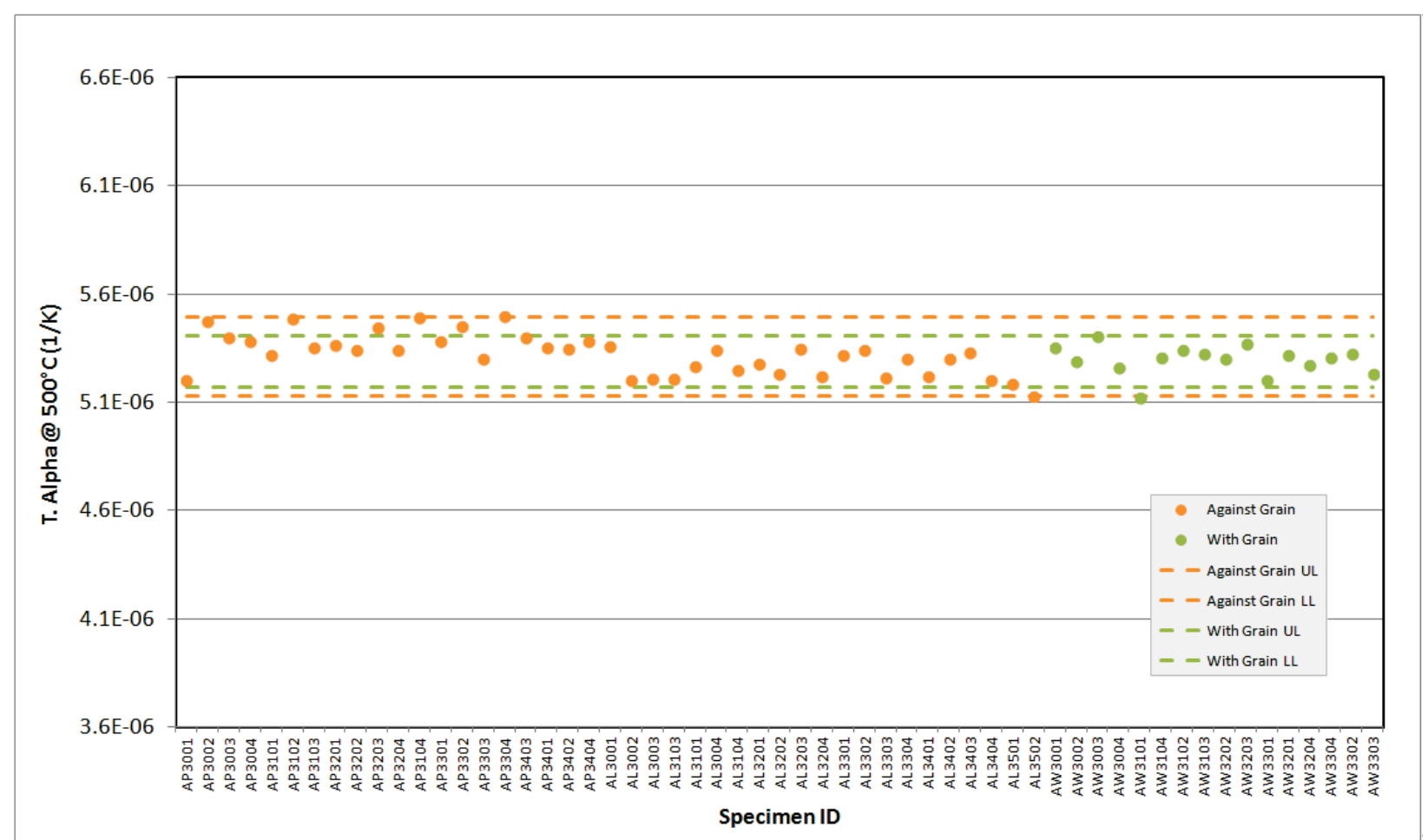

Figure A-51. NBG-17 Creep Coefficient of Thermal Expansion @ $500^{\circ} \mathrm{C}$. 


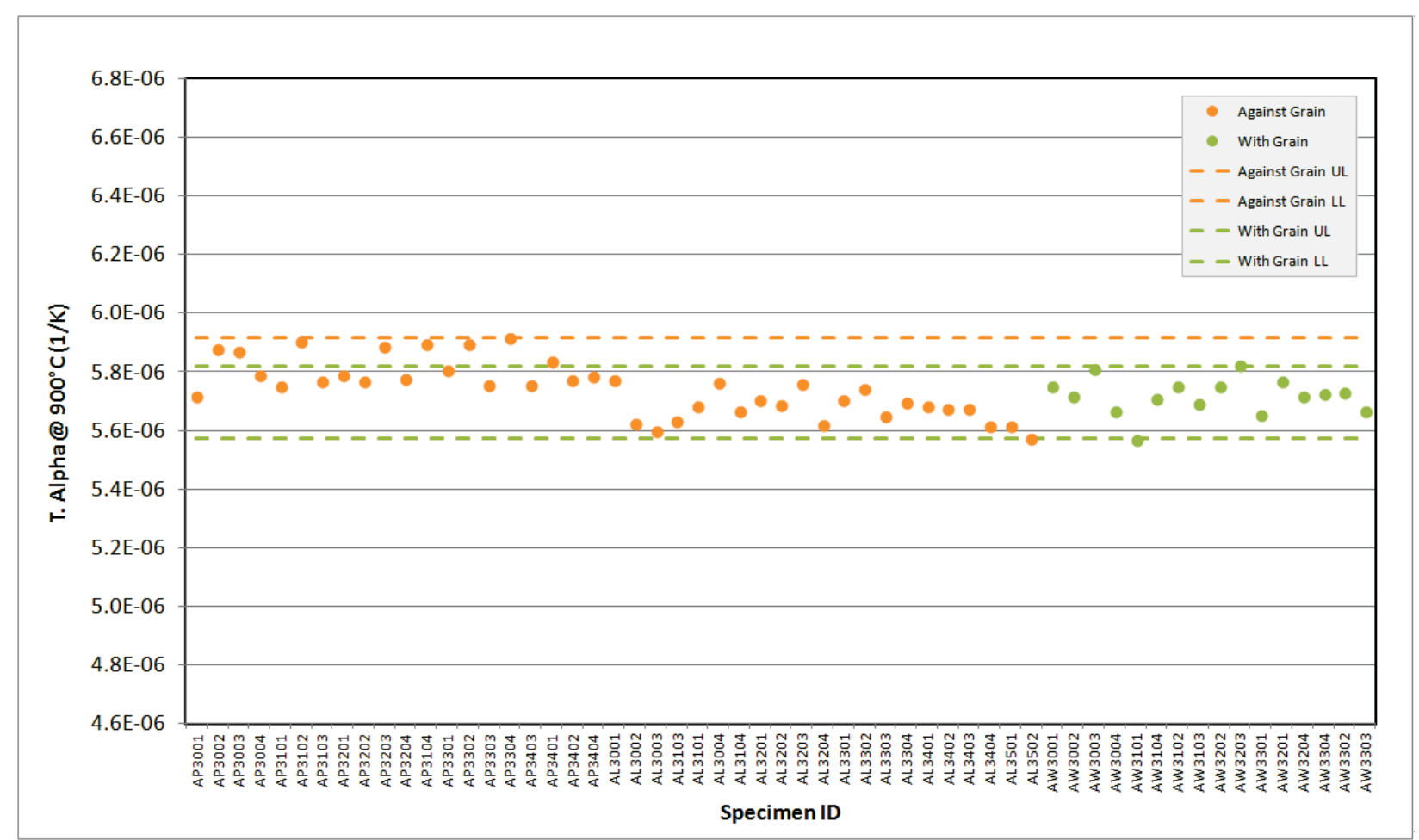

Figure A-52. NBG-17 Creep Coefficient of Thermal Expansion @ $900^{\circ} \mathrm{C}$.

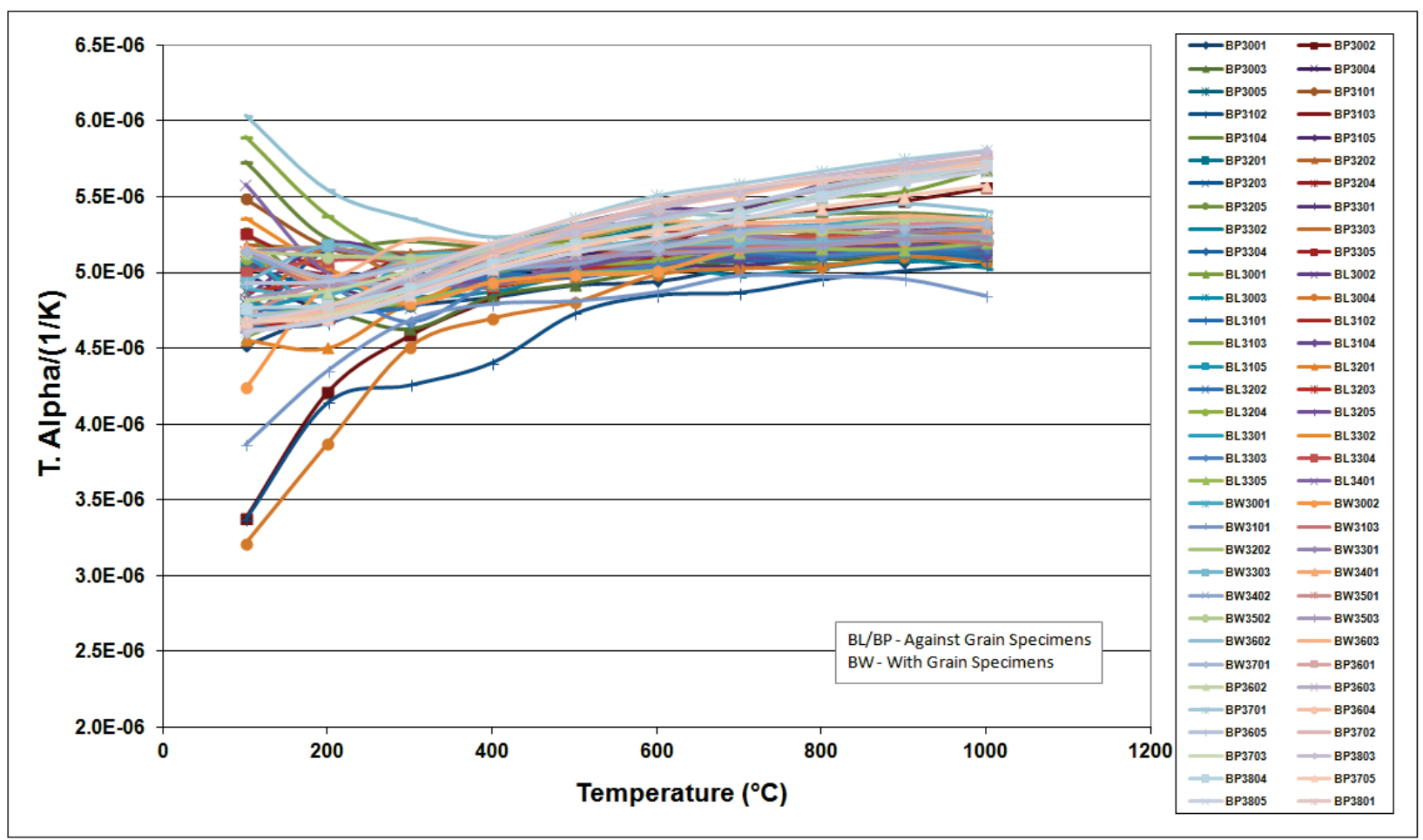

Figure A-53. NBG-18 Creep Coefficient of Thermal Expansion. 


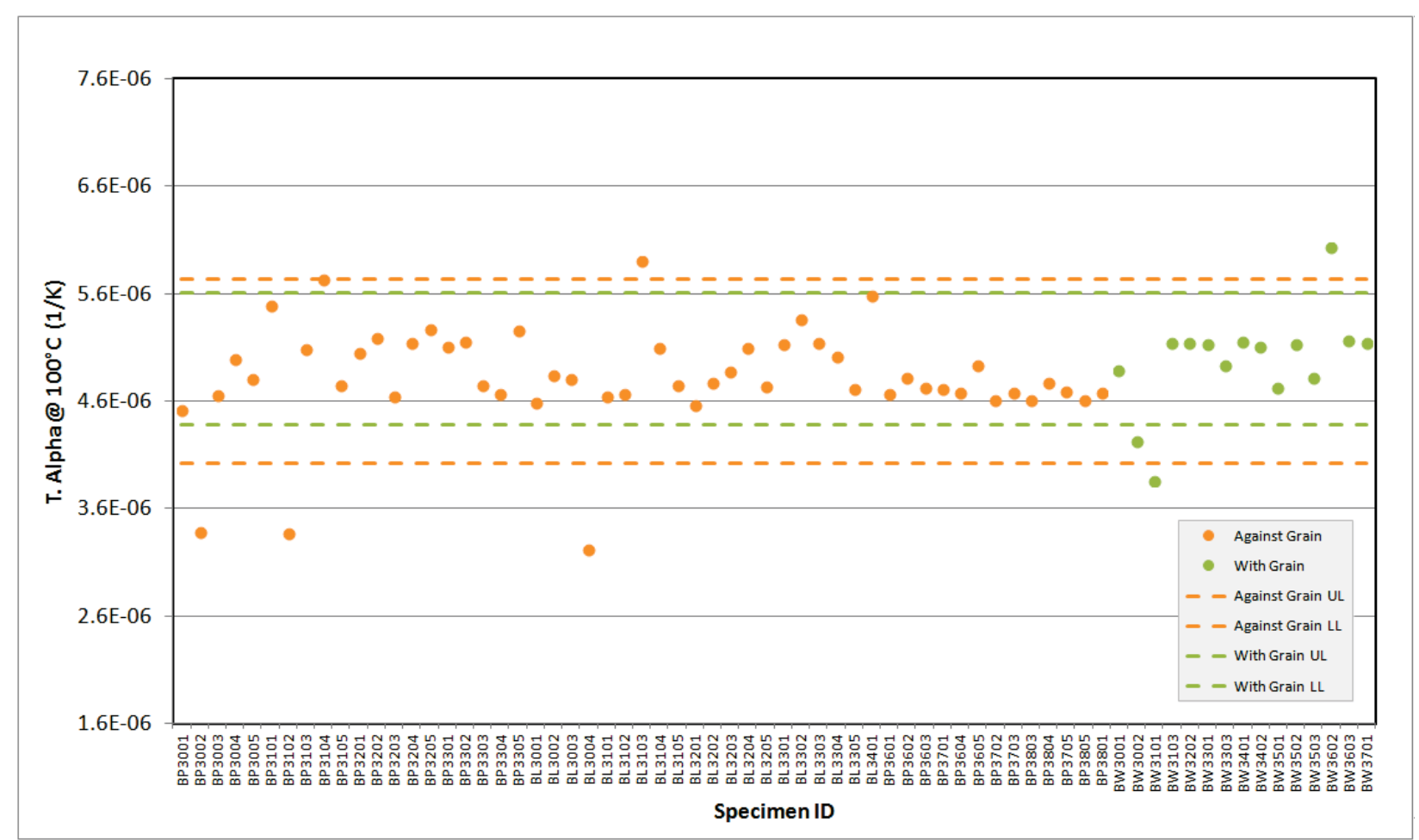

Figure A-54. NBG-18 Creep Coefficient of Thermal Expansion @ $100^{\circ} \mathrm{C}$.

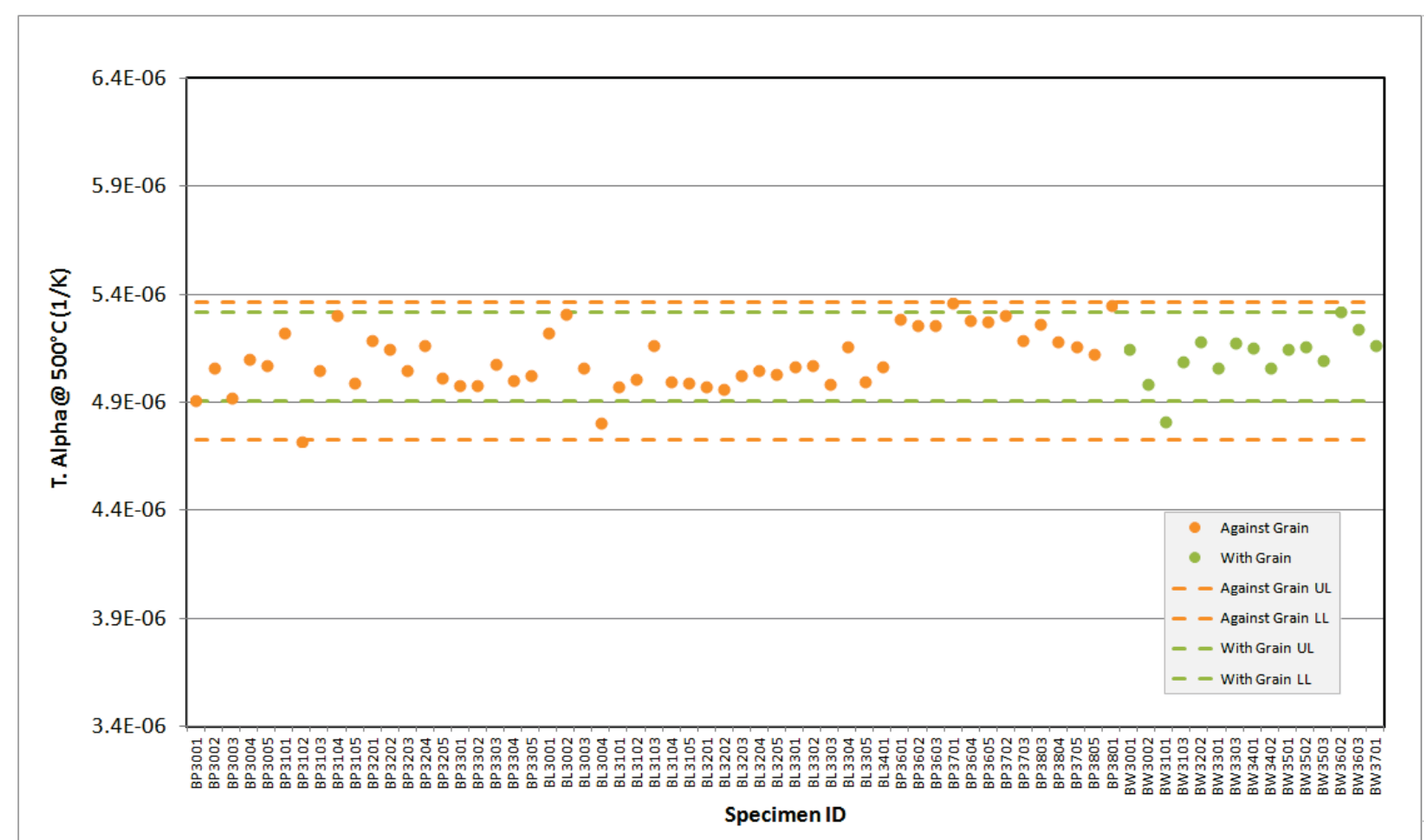

Figure A-55. NBG-18 Creep Coefficient of Thermal Expansion @ $500^{\circ} \mathrm{C}$. 


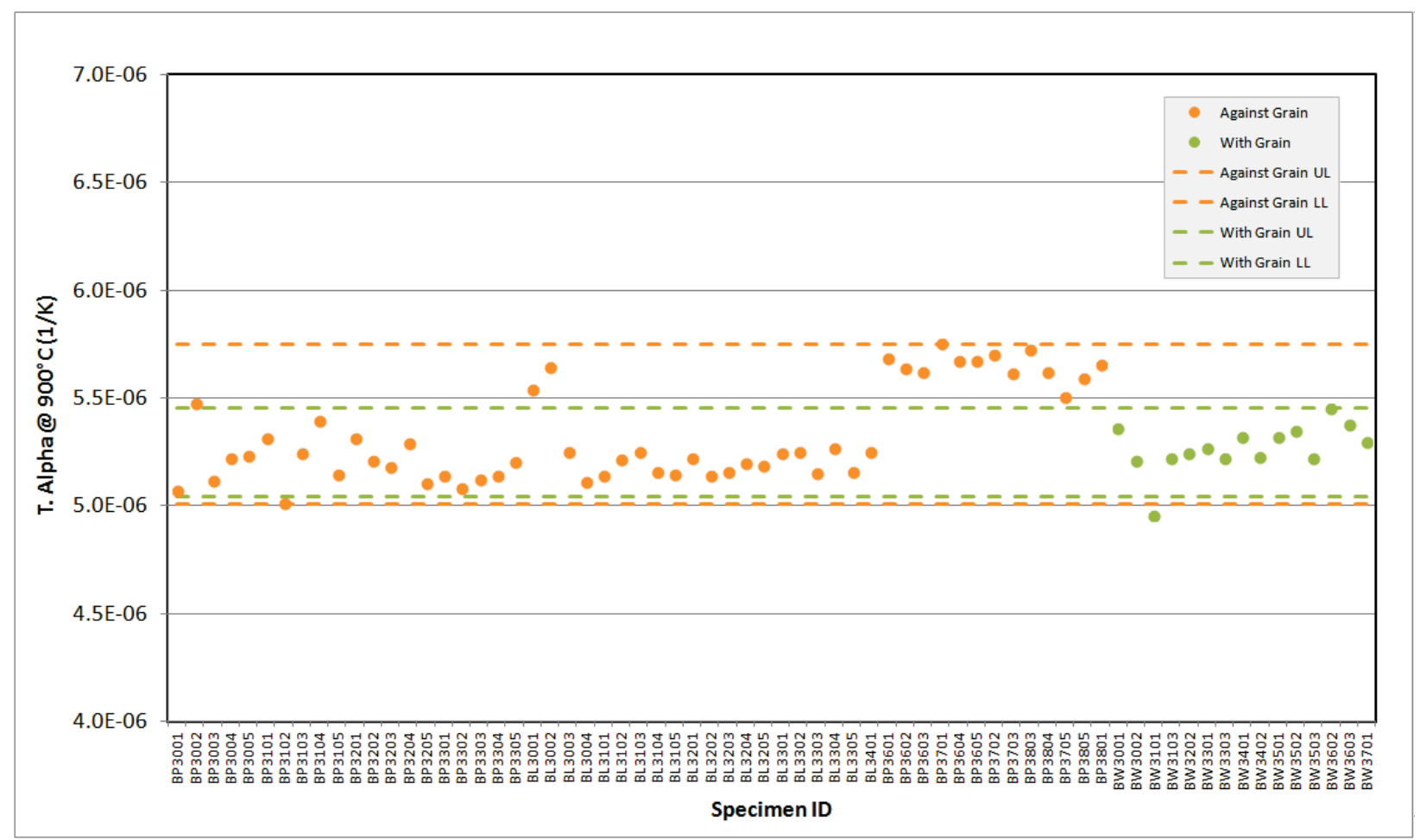

Figure A-56. NBG-18 Creep Coefficient of Thermal Expansion @ $900^{\circ} \mathrm{C}$.

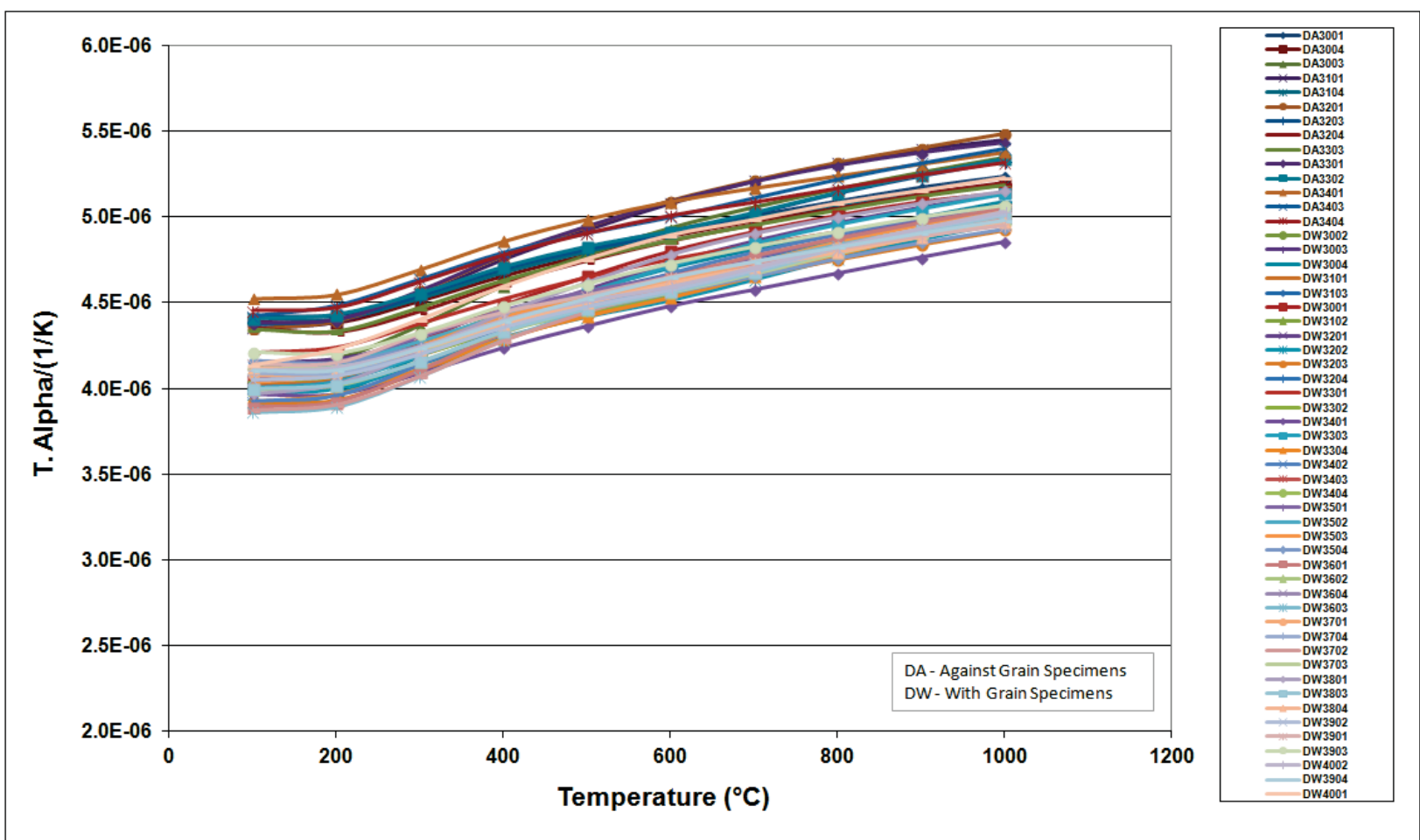

Figure A-57. PCEA Creep Coefficient of Thermal Expansion. 


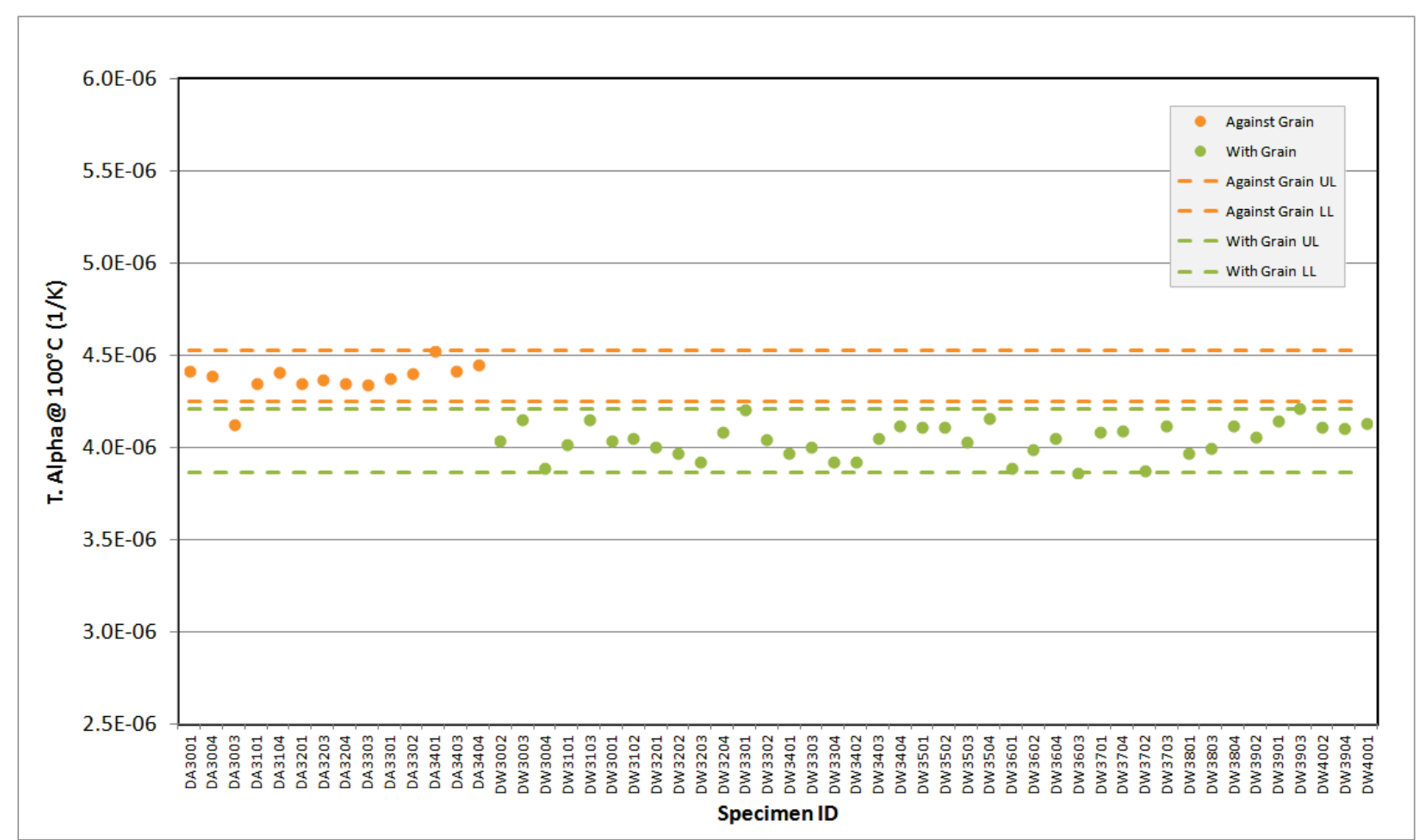

Figure A-58. PCEA Creep Coefficient of Thermal Expansion @ $100^{\circ} \mathrm{C}$.

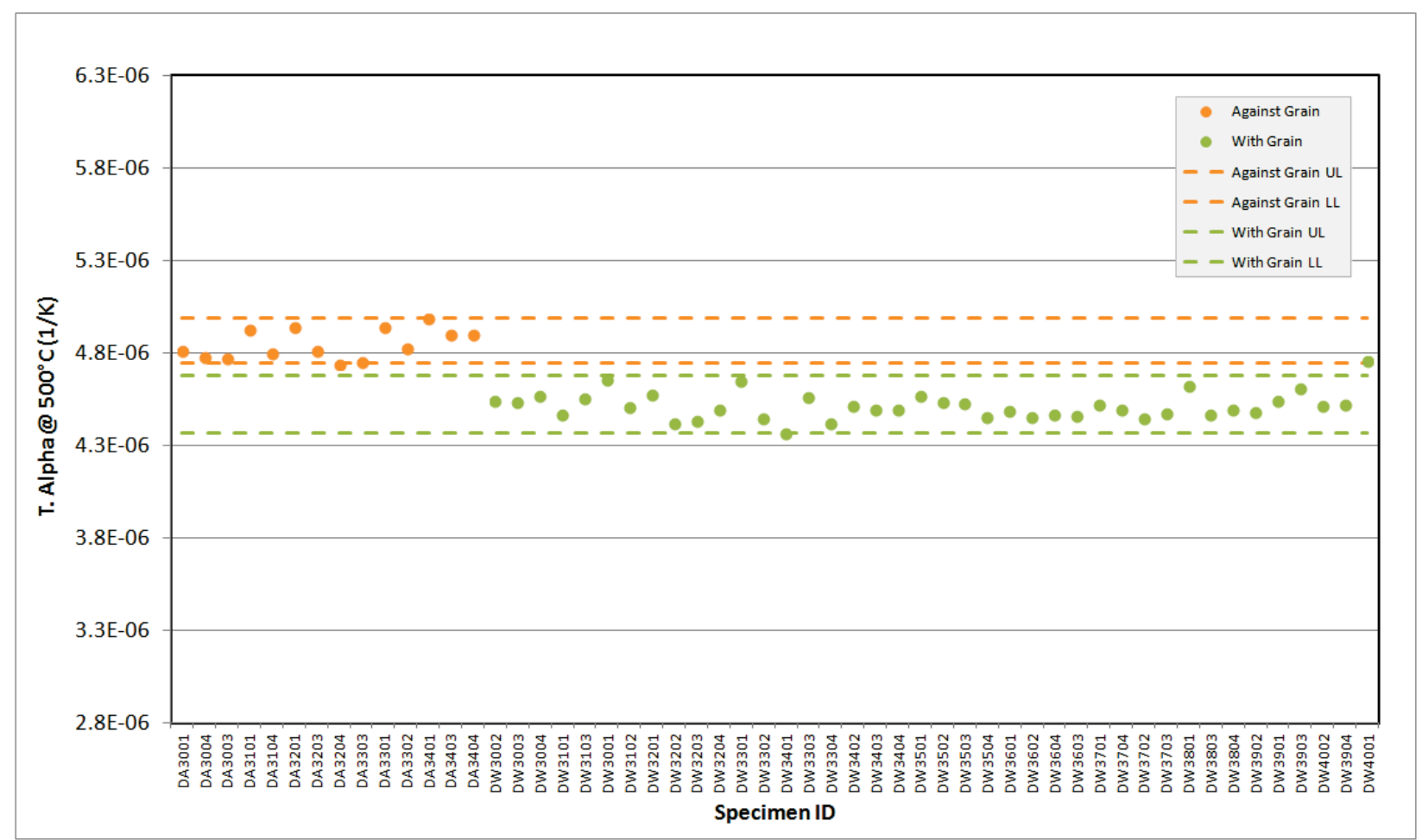

Figure A-59. PCEA Creep Coefficient of Thermal Expansion @ $500^{\circ} \mathrm{C}$. 


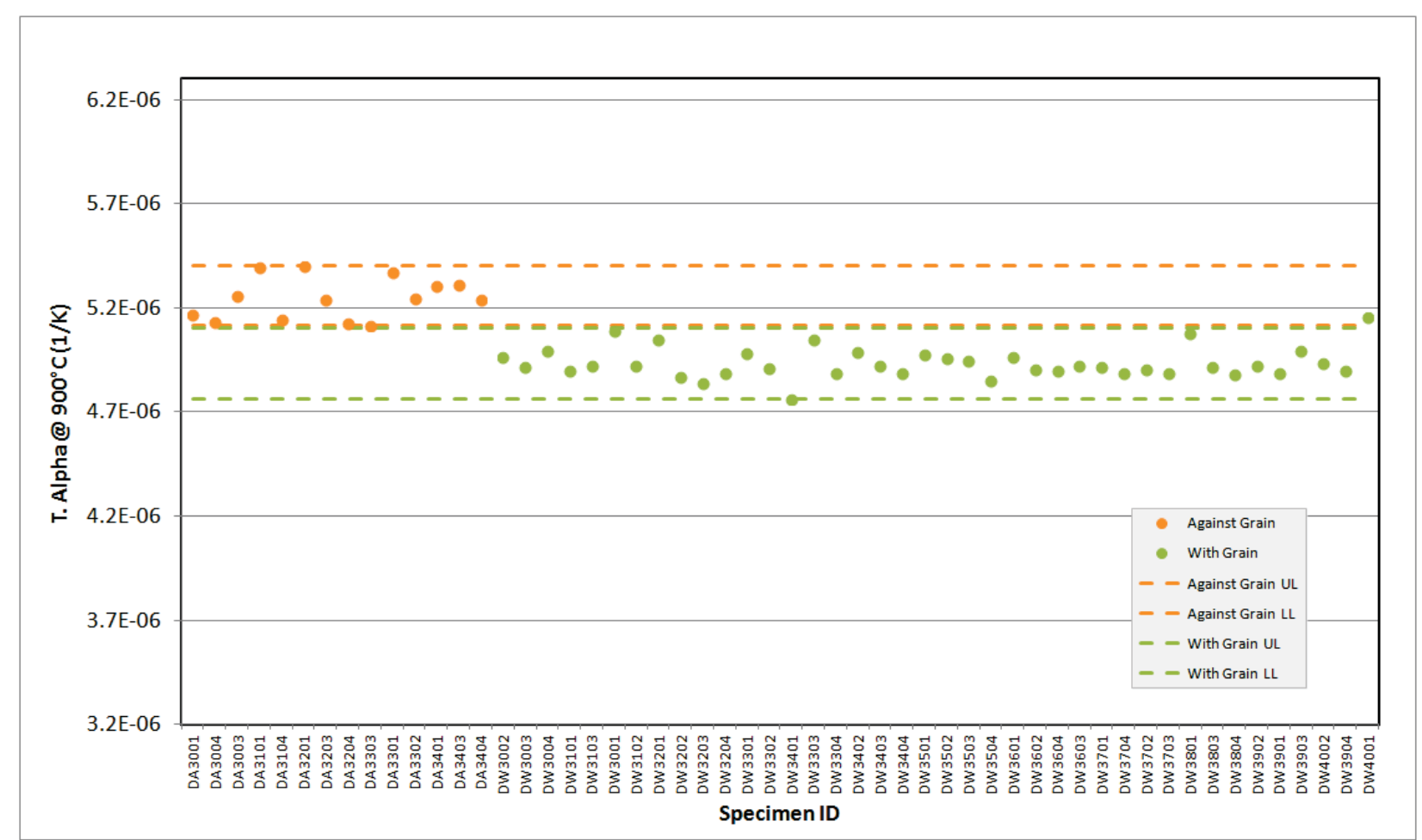

Figure A-60. PCEA Creep Coefficient of Thermal Expansion@ $900^{\circ} \mathrm{C}$.

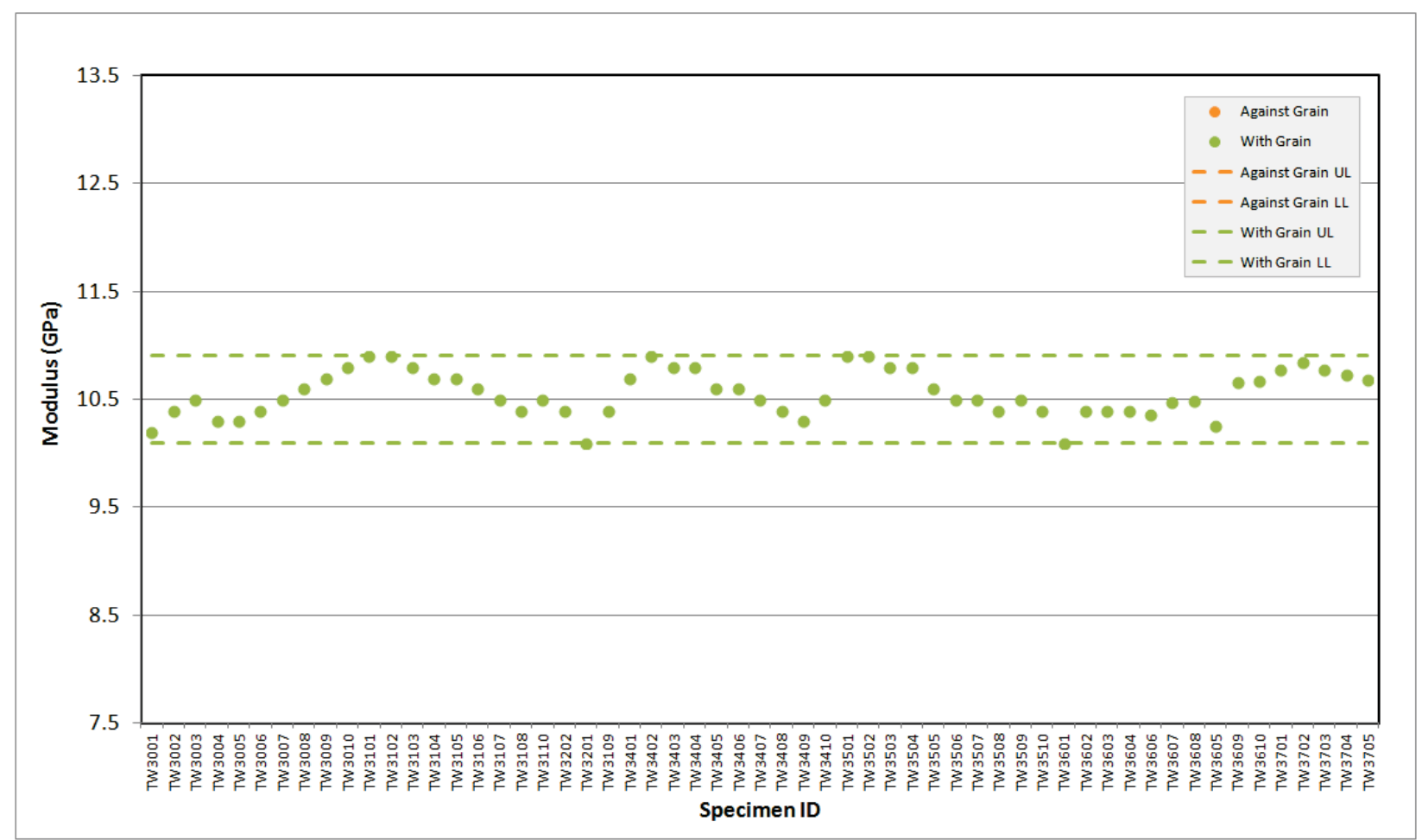

Figure A-61. 2114 Creep Modulus by Sonic Resonance. 


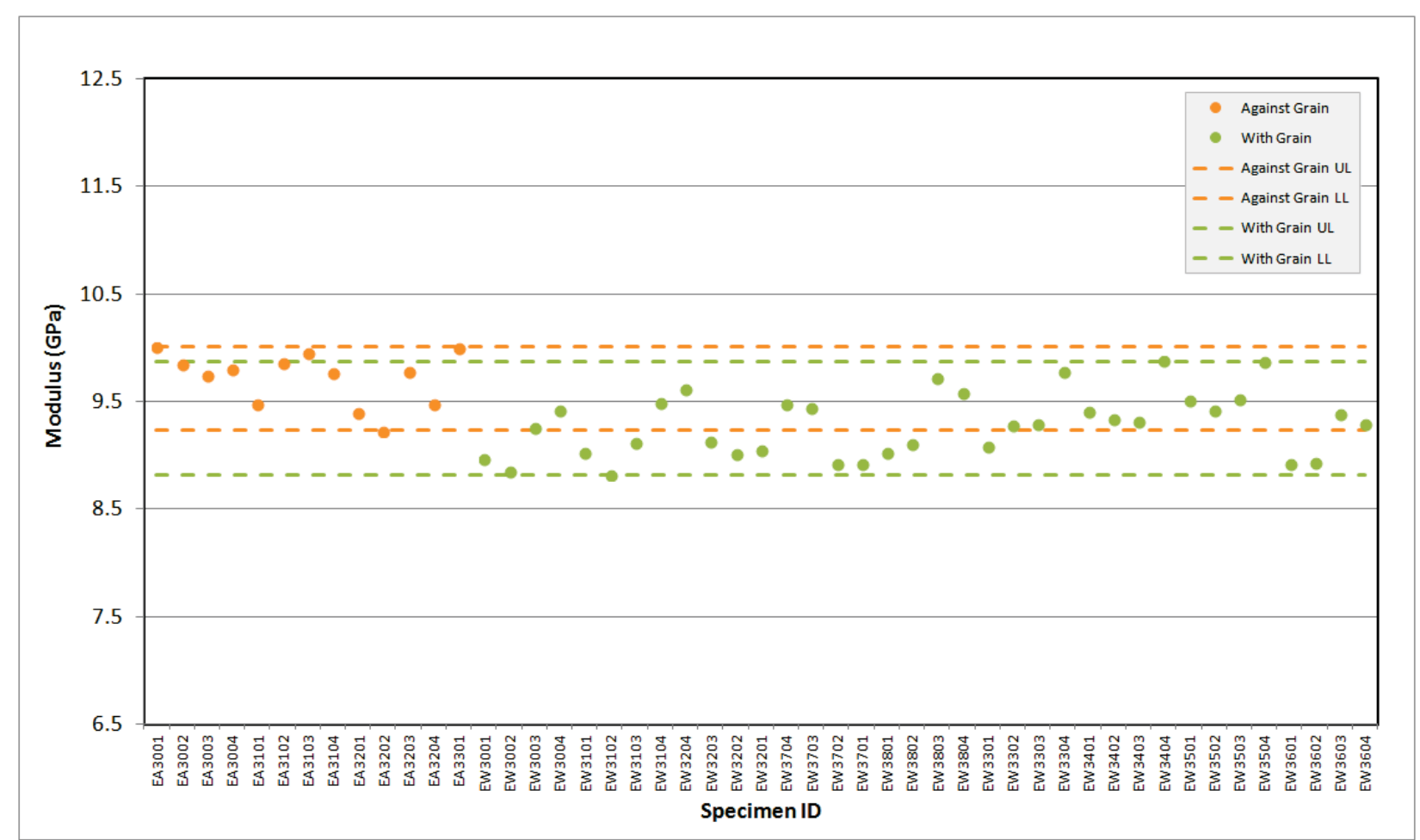

Figure A-62. IG-110 Creep Modulus by Sonic Resonance.

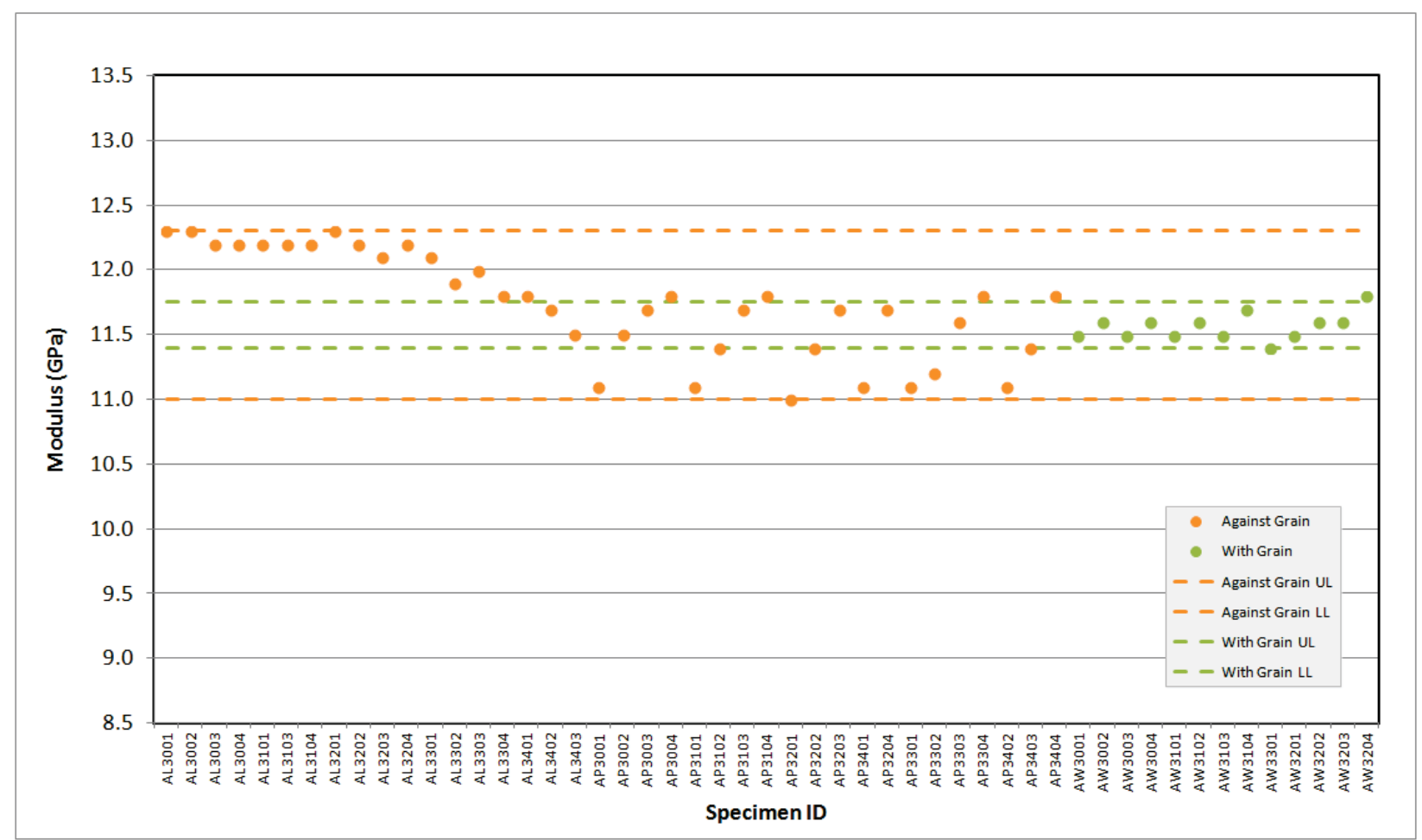

Figure A-63. NBG-17 Creep Modulus by Sonic Resonance. 


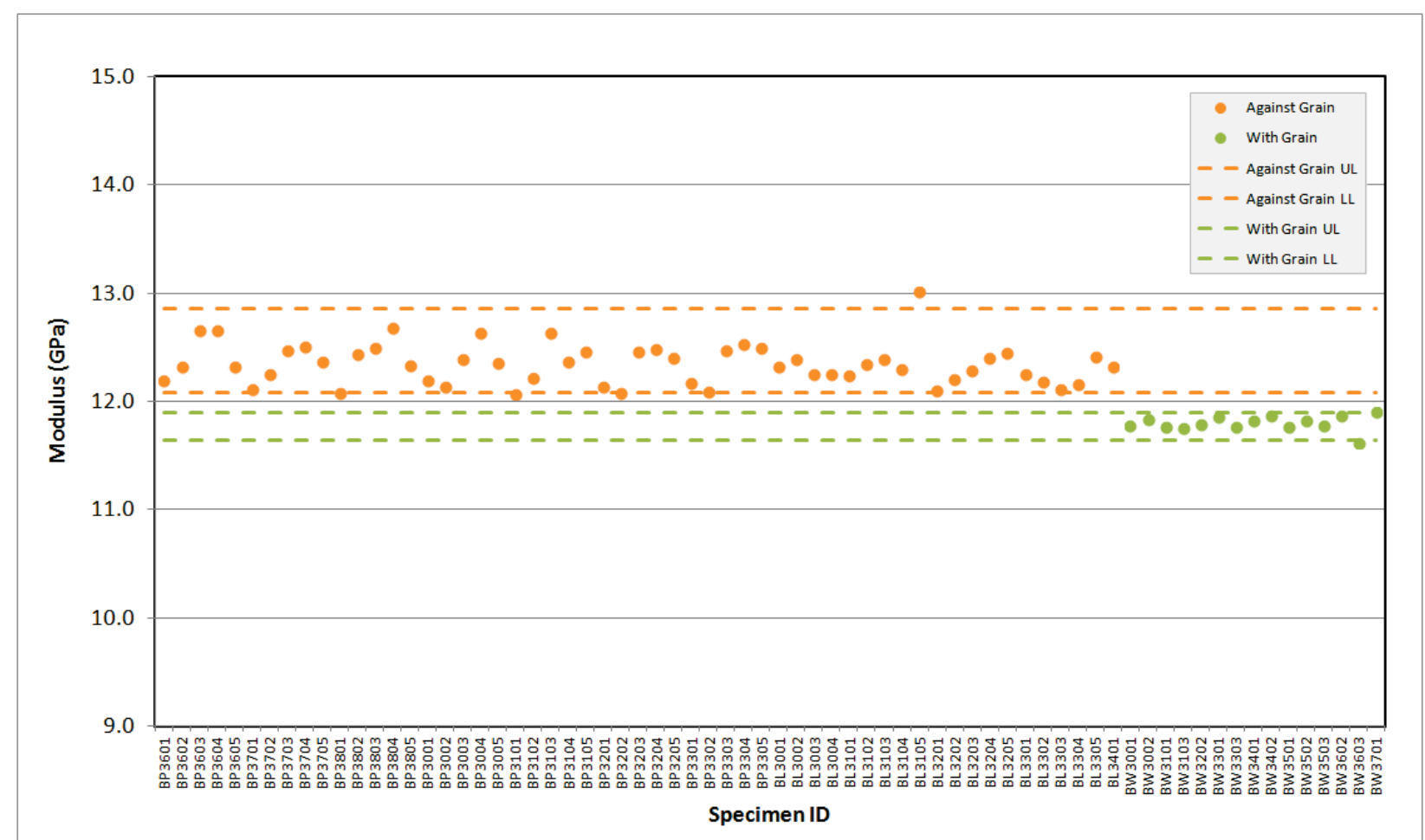

Figure A-64. NBG-18 Creep Modulus by Sonic Resonance.

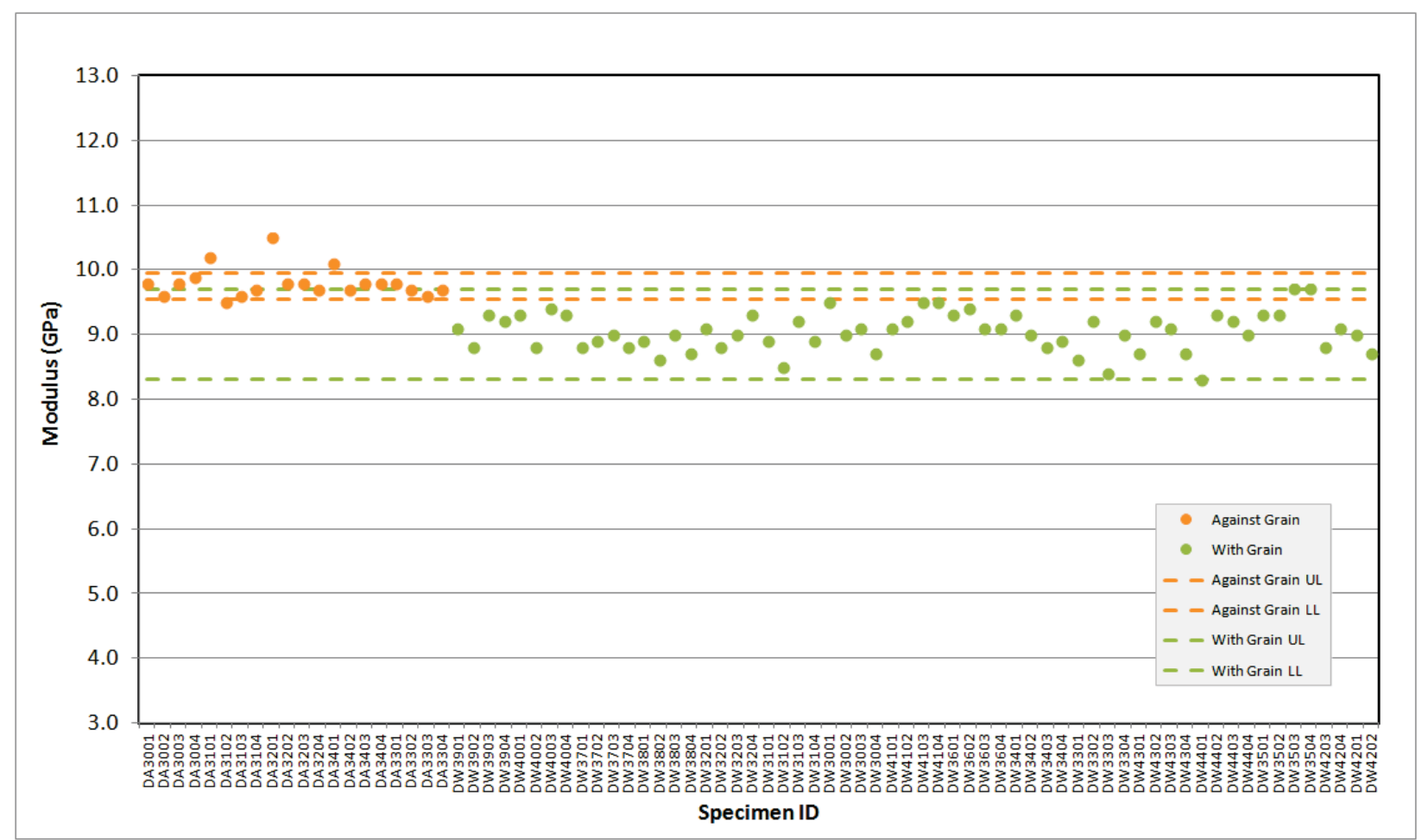

Figure A-65. PCEA Creep Modulus by Sonic Resonance. 


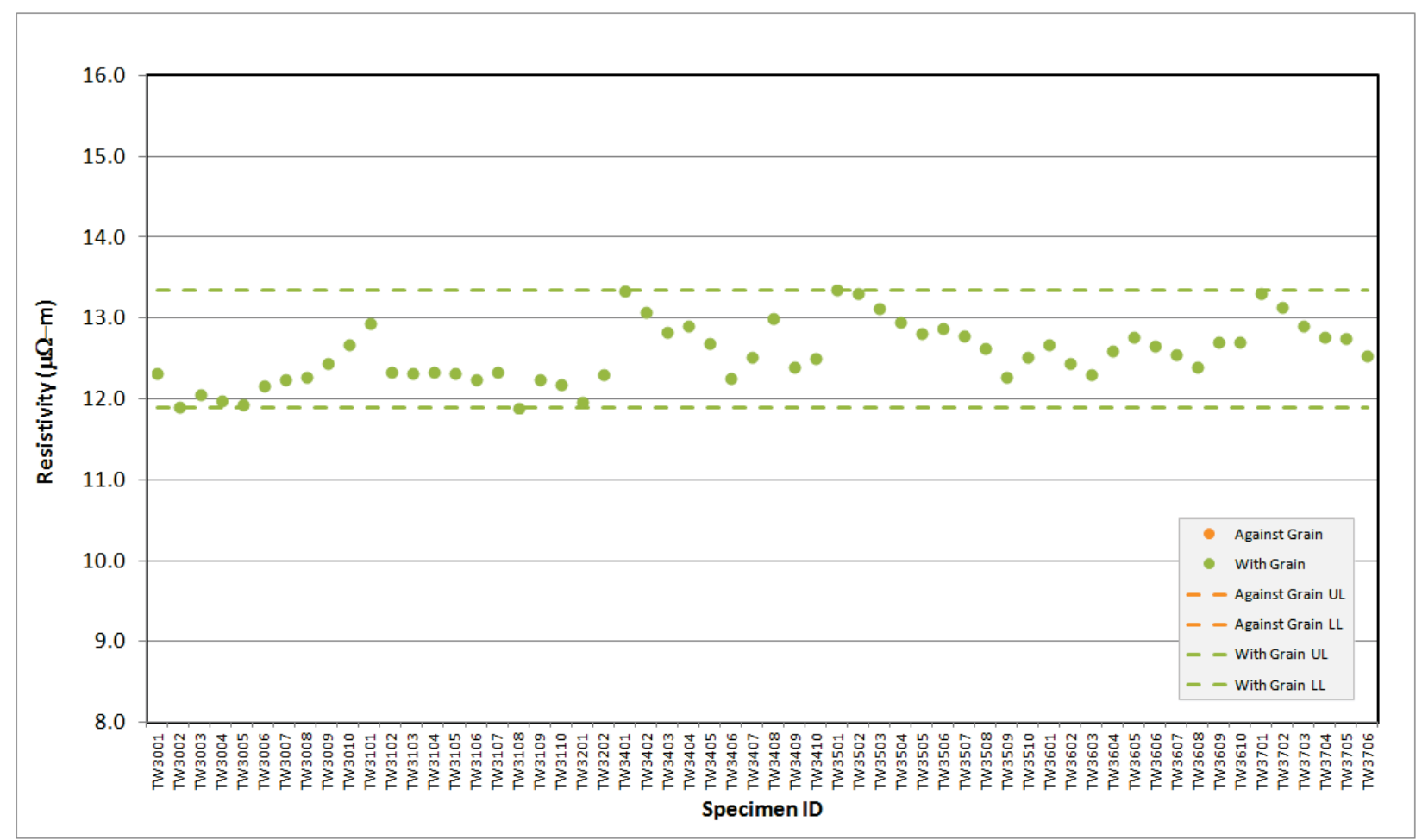

Figure A-66. 2114 Creep Resistivity.

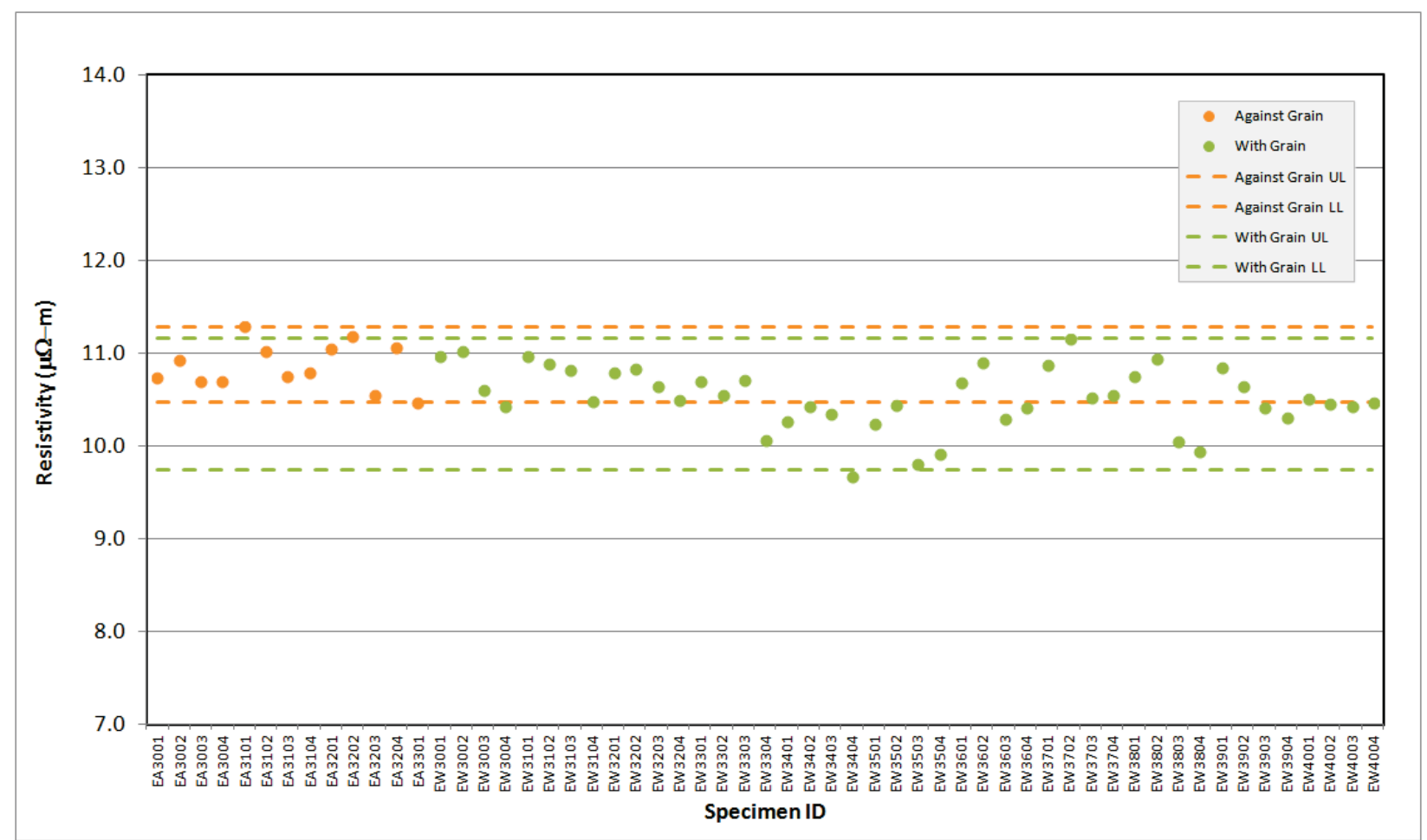

Figure A-67. IG-110 Creep Resistivity. 


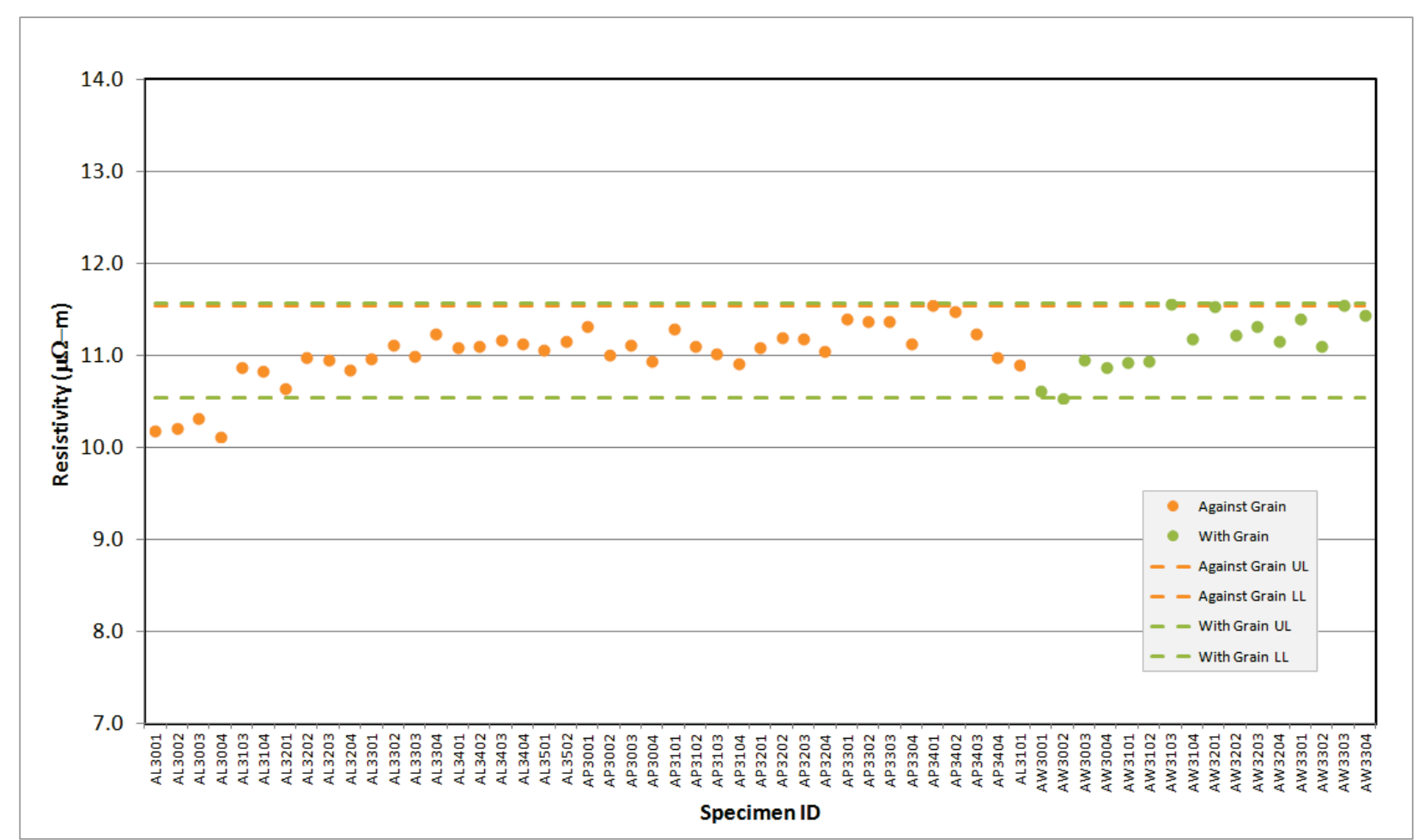

Figure A-68. NBG-17 Creep Resistivity.

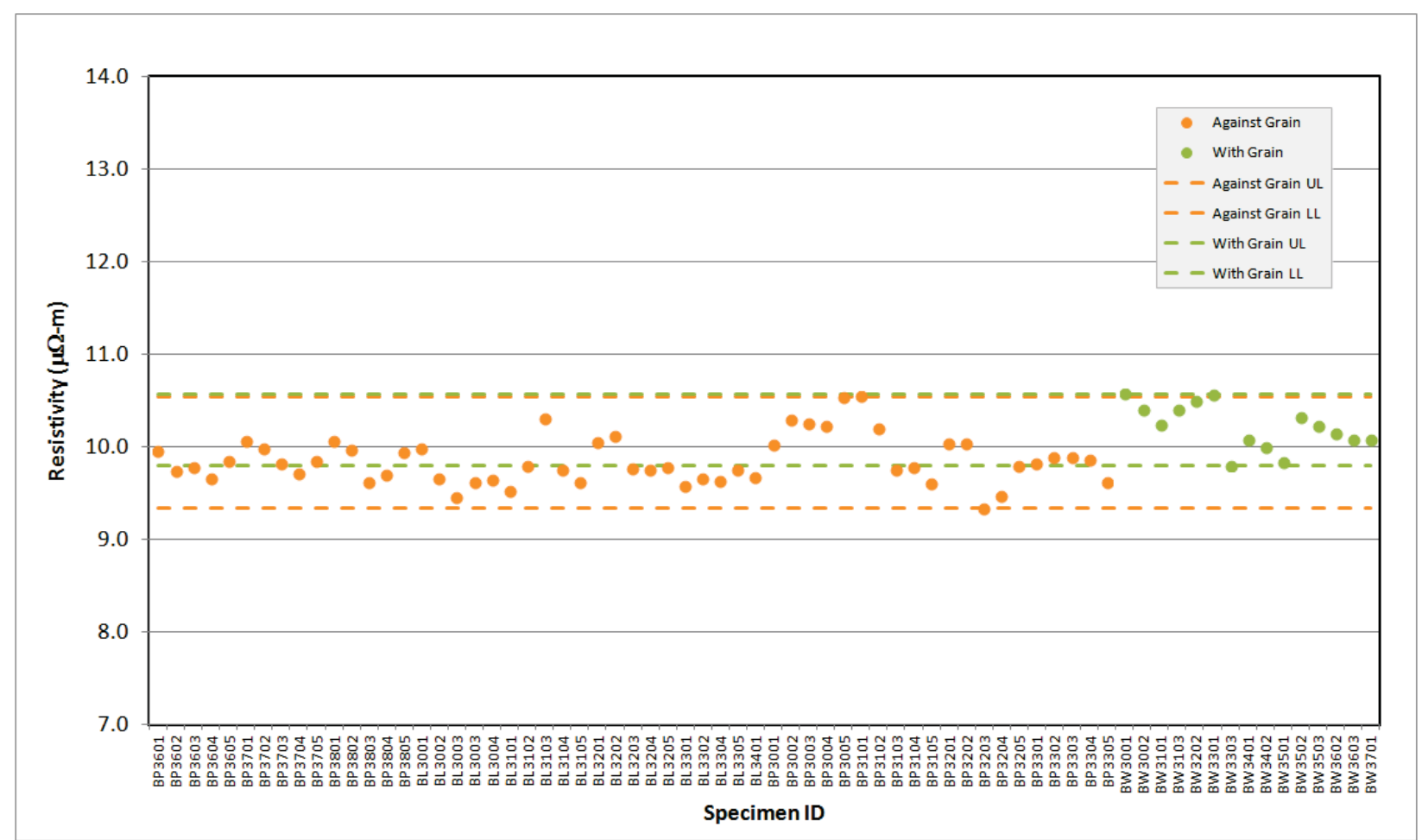

Figure A-69. NBG-18 Creep Resistivity. 


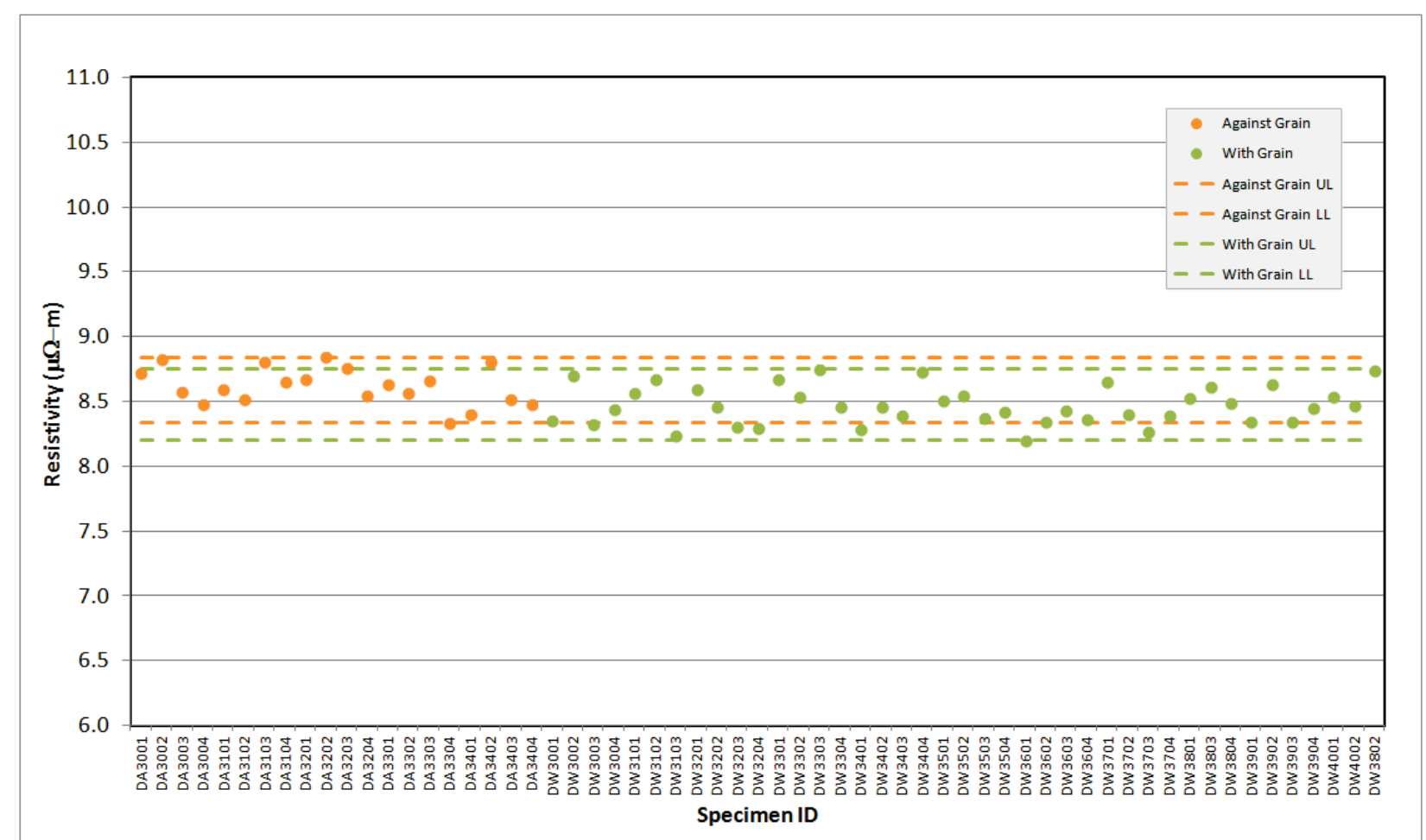

Figure A-70. PCEA Creep Resistivity.

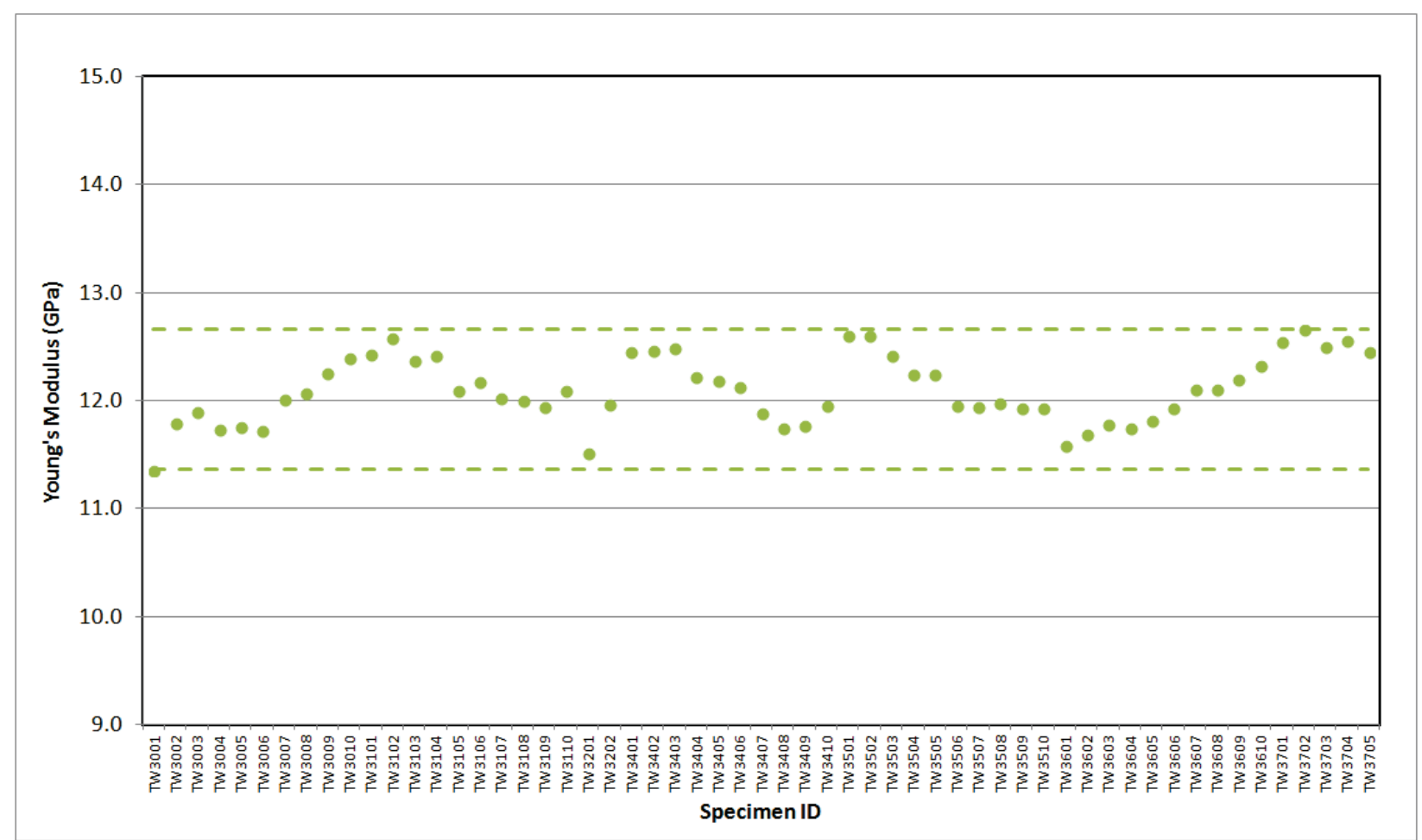

Figure A-71. 2114 Creep Young's Modulus by Sonic Velocity. 


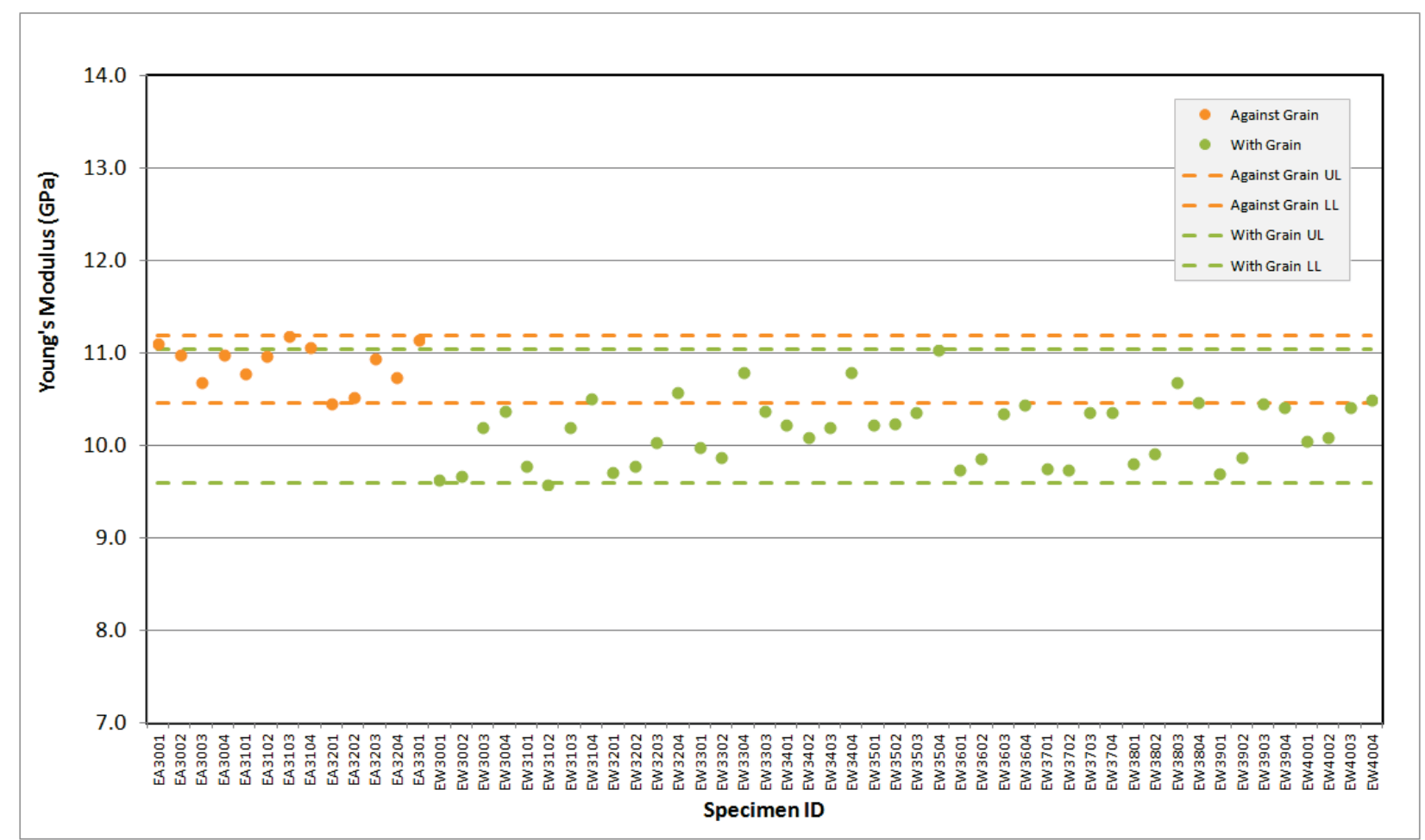

Figure A-72. IG-110 Creep Young's Modulus by Sonic Velocity.

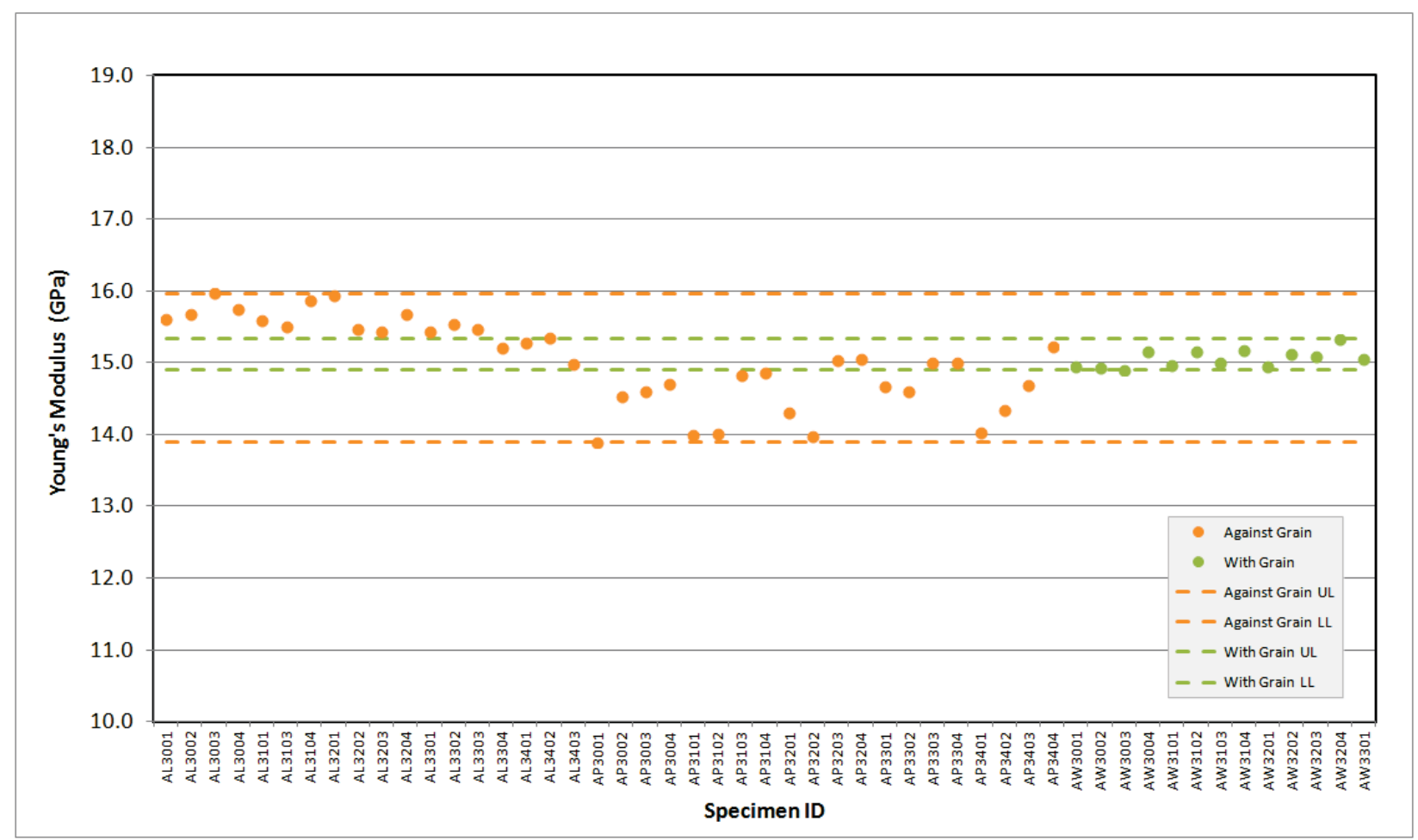

Figure A-73. NBG-17 Creep Young's Modulus by Sonic Velocity. 


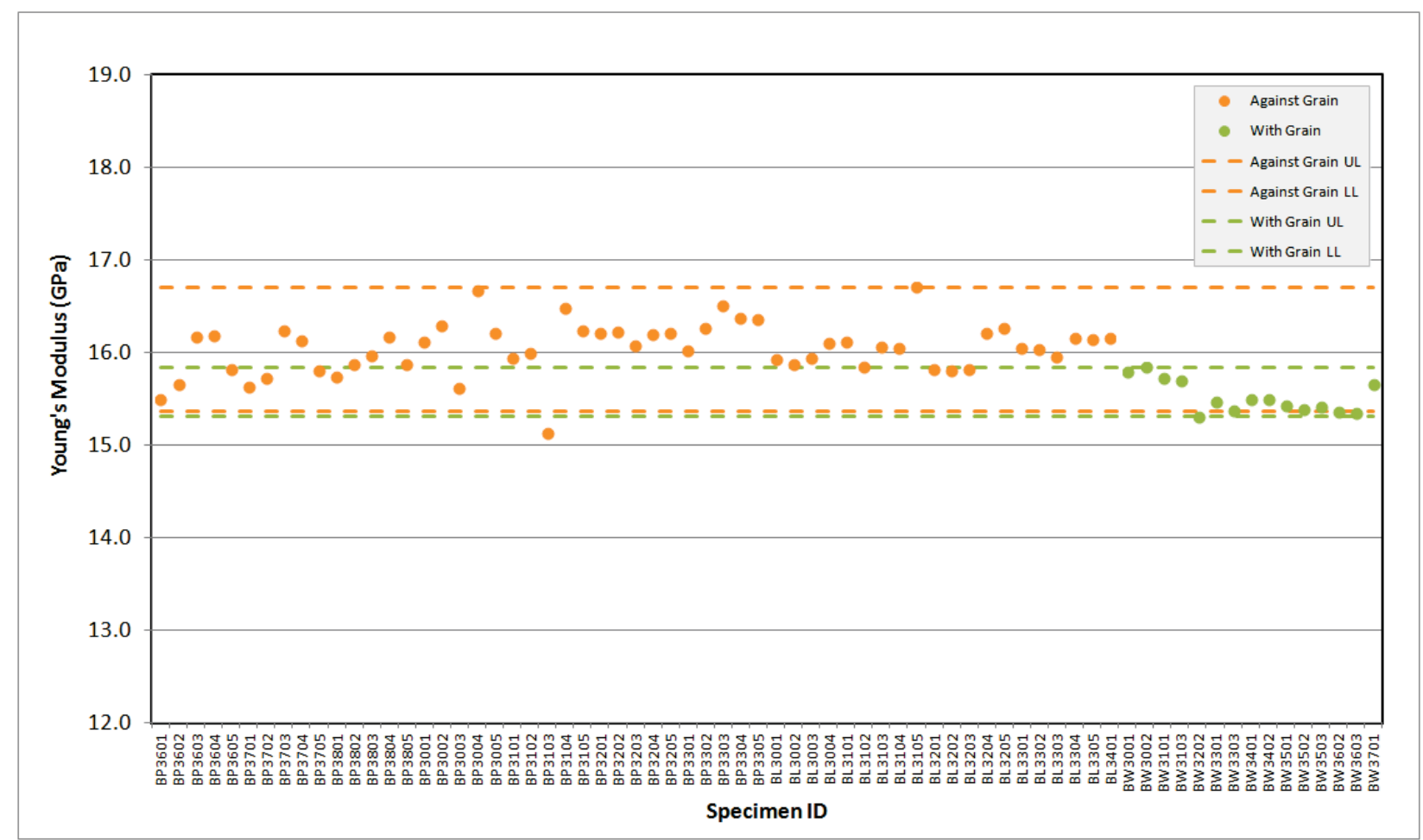

Figure A-74. NBG-18 Creep Young's Modulus by Sonic Velocity.

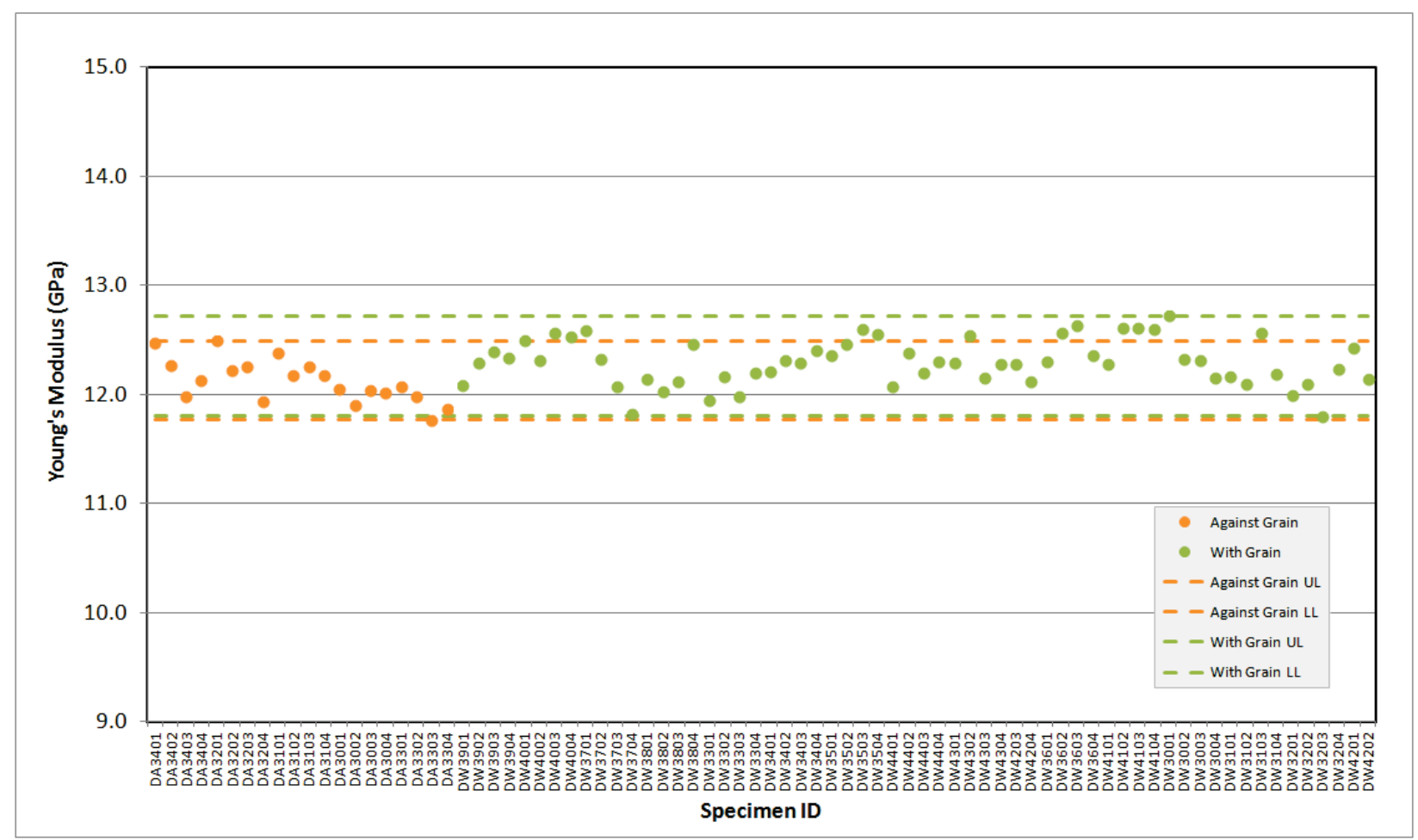

Figure A-75. PCEA Creep Young's Modulus by Sonic Velocity. 


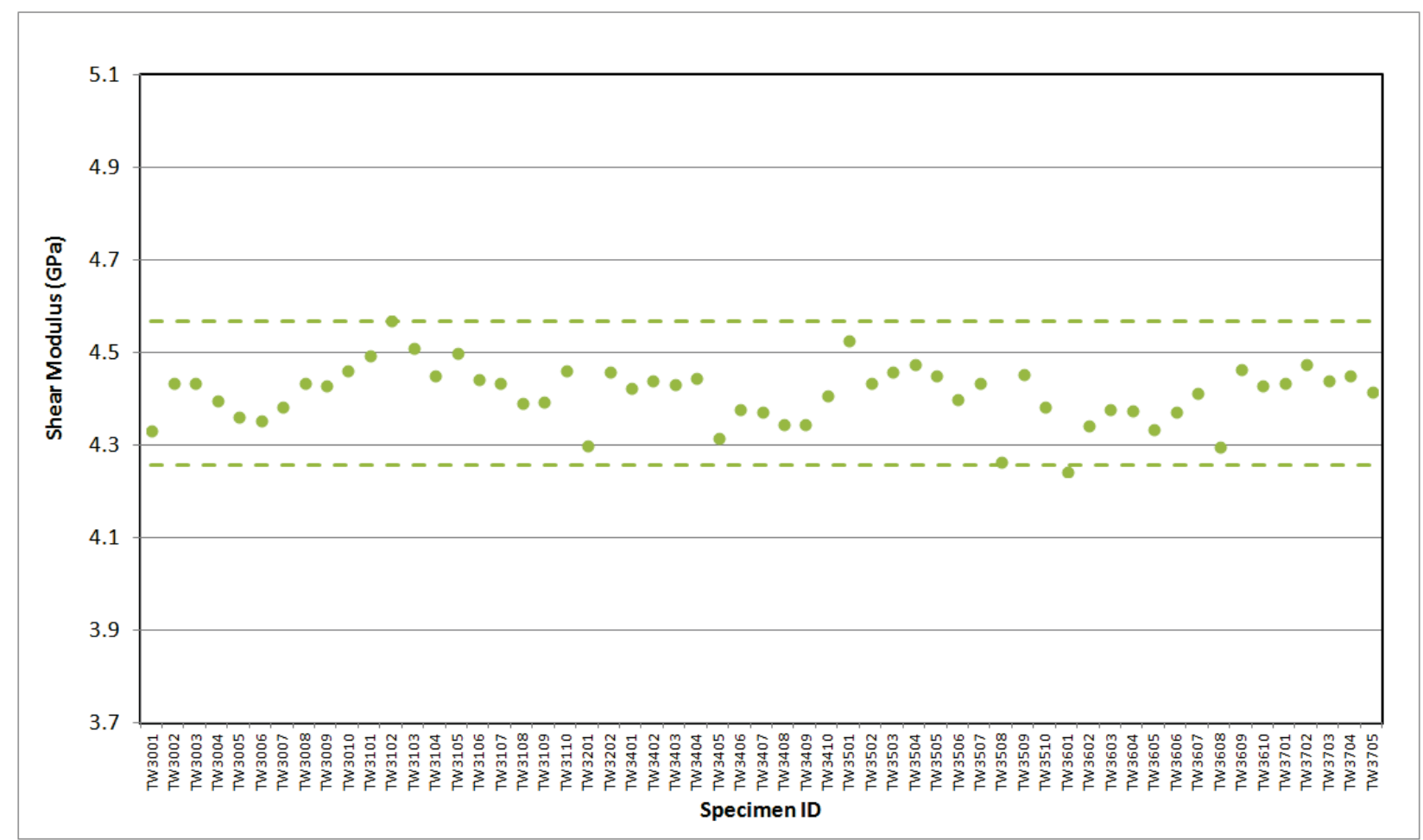

Figure A-76. 2114 Creep Shear Modulus by Sonic Velocity.

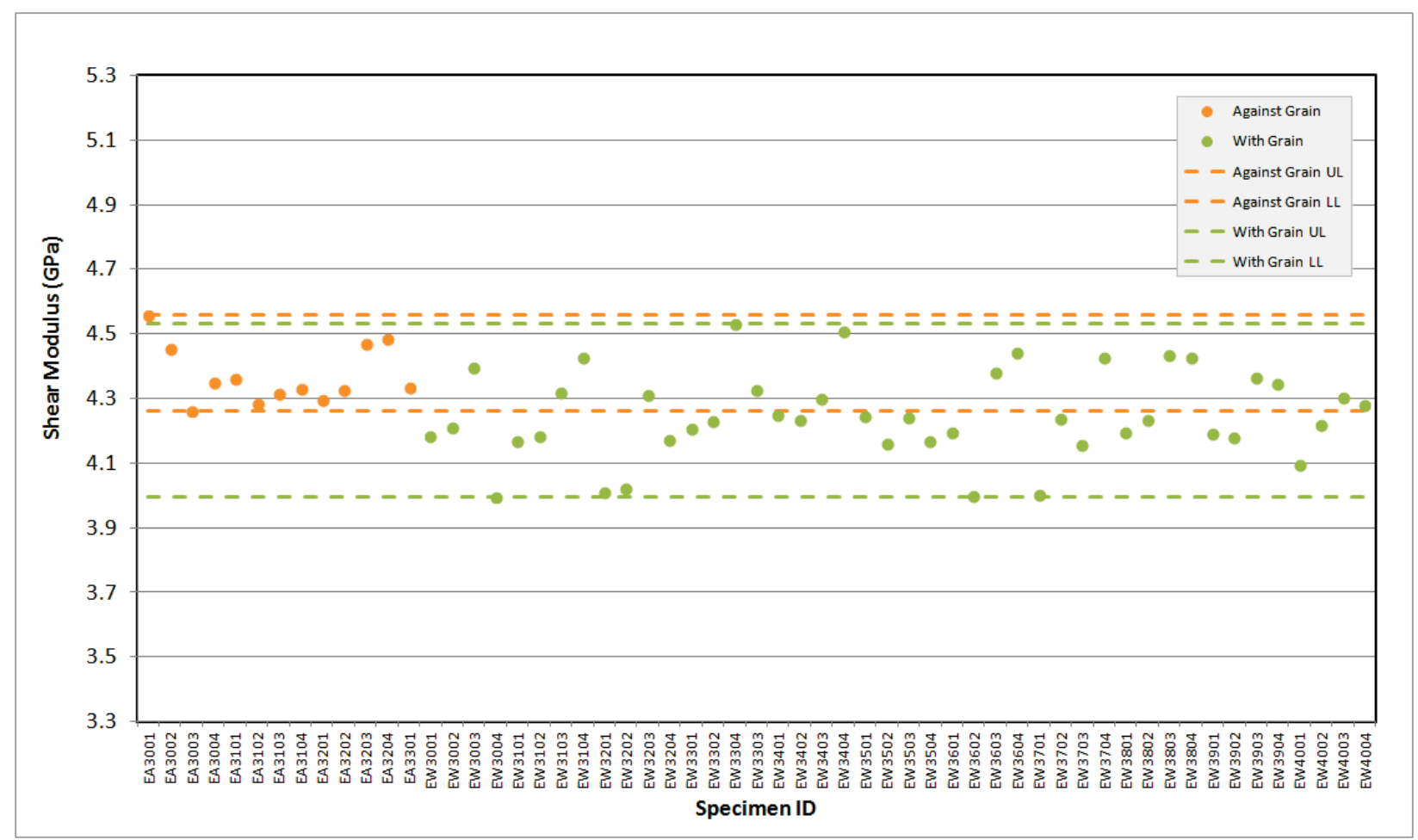

Figure A-77. IG-110 Creep Shear Modulus by Sonic Velocity. 


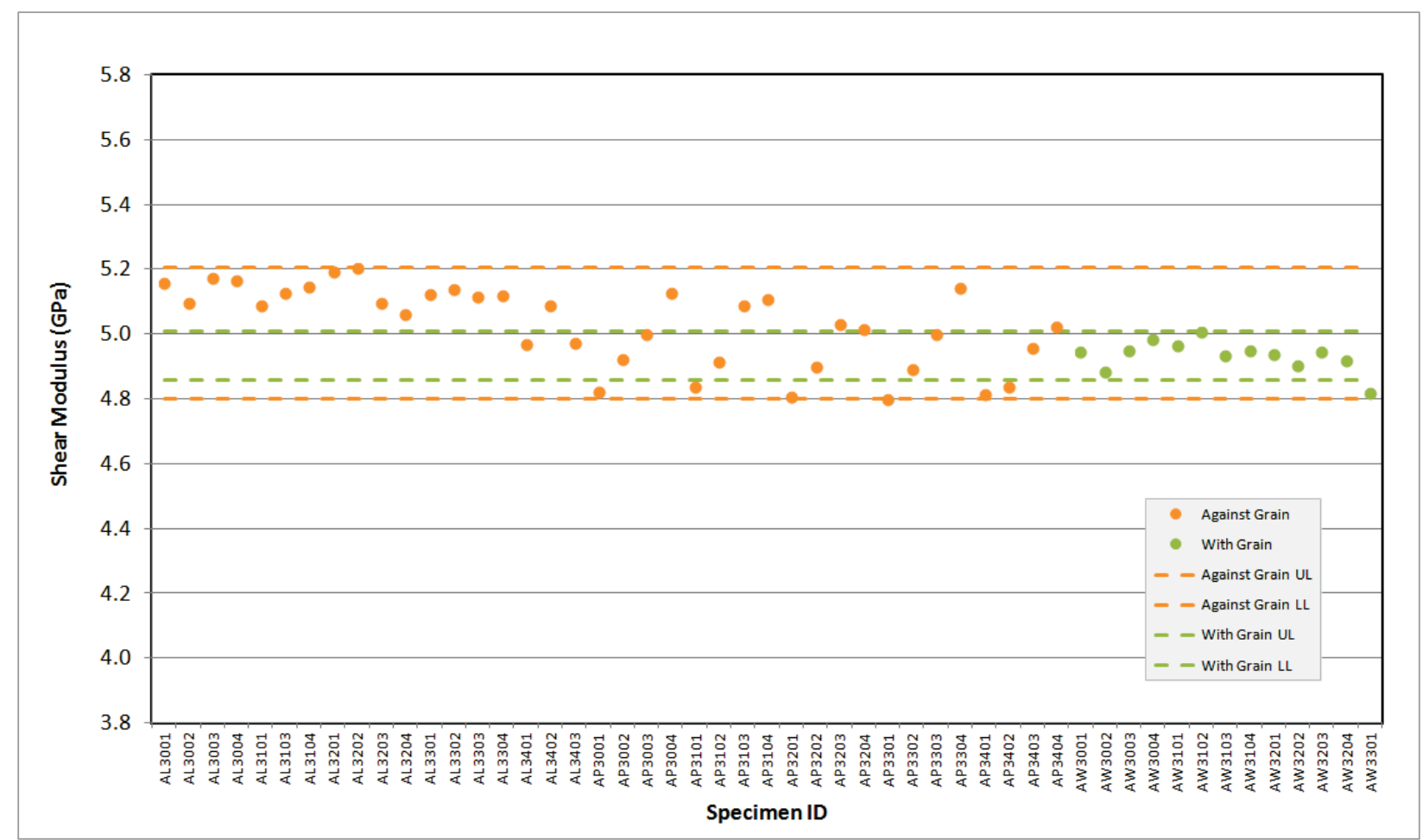

Figure A-78. NBG-17 Creep Shear Modulus by Sonic Velocity.

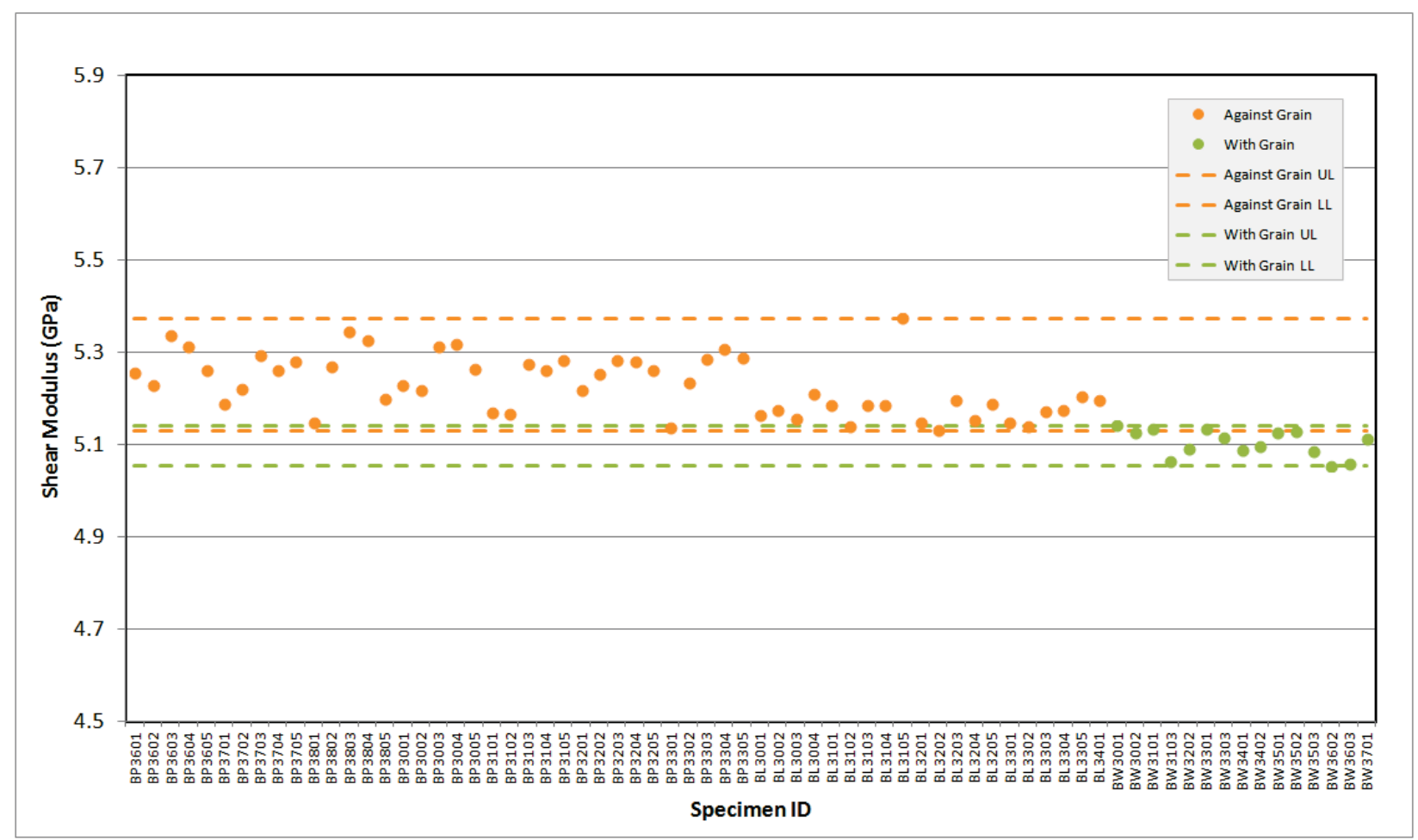

Figure A-79. NBG-18 Creep Shear Modulus by Sonic Velocity. 


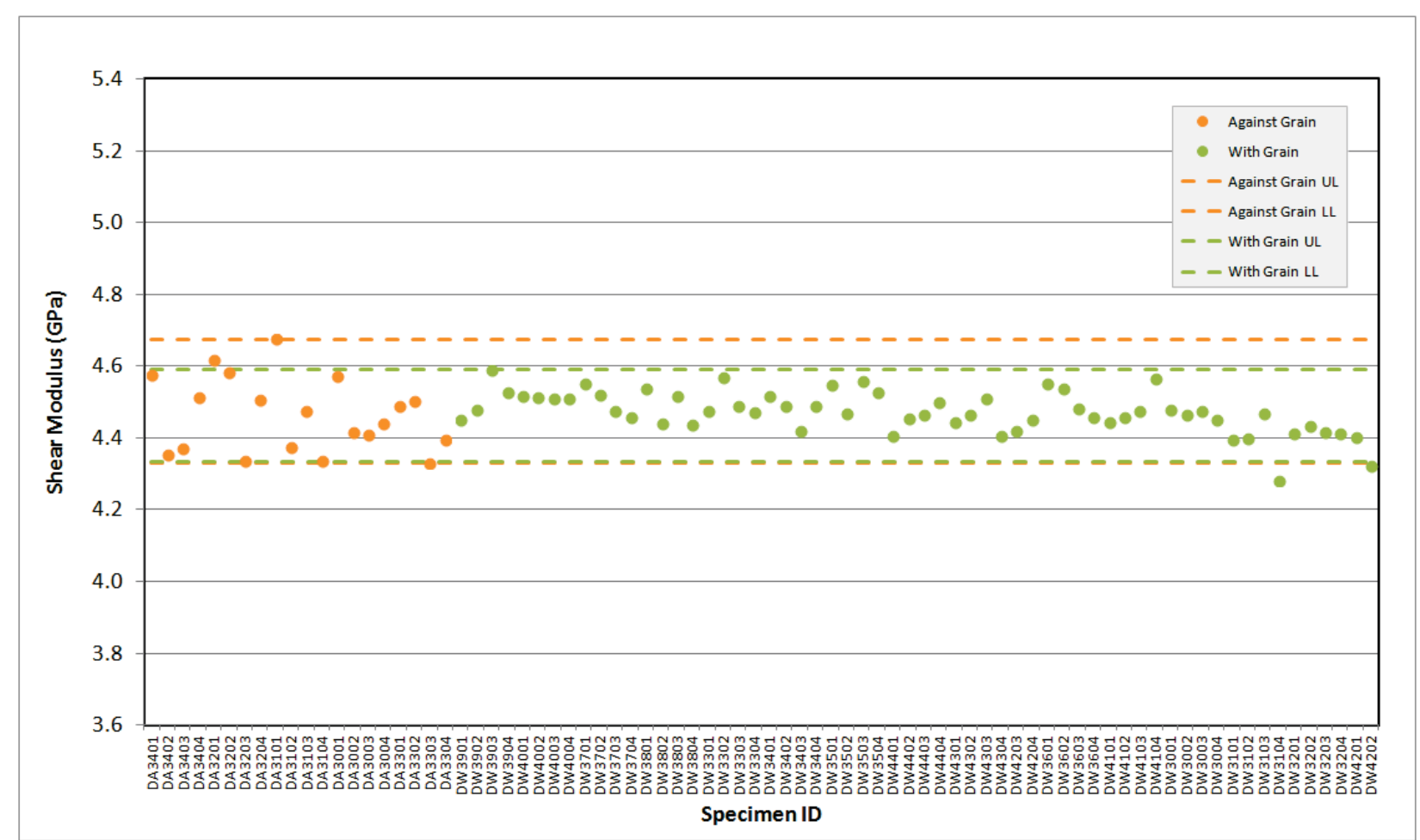

Figure A-80. PCEA Creep Shear Modulus by Sonic Velocity.

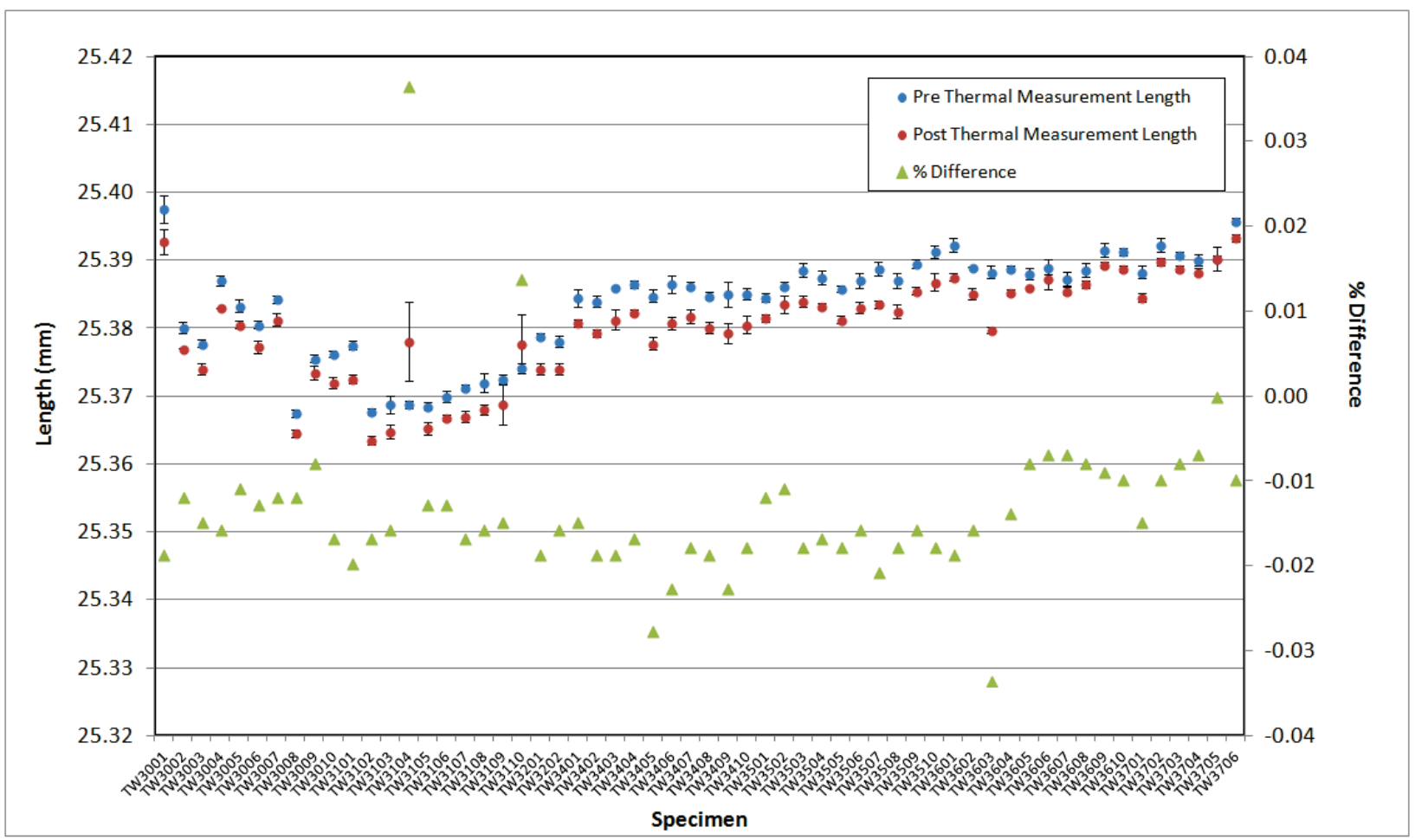

Figure A-81. 2114 Creep Pre vs. Post Thermal Measurement Length Comparison. 


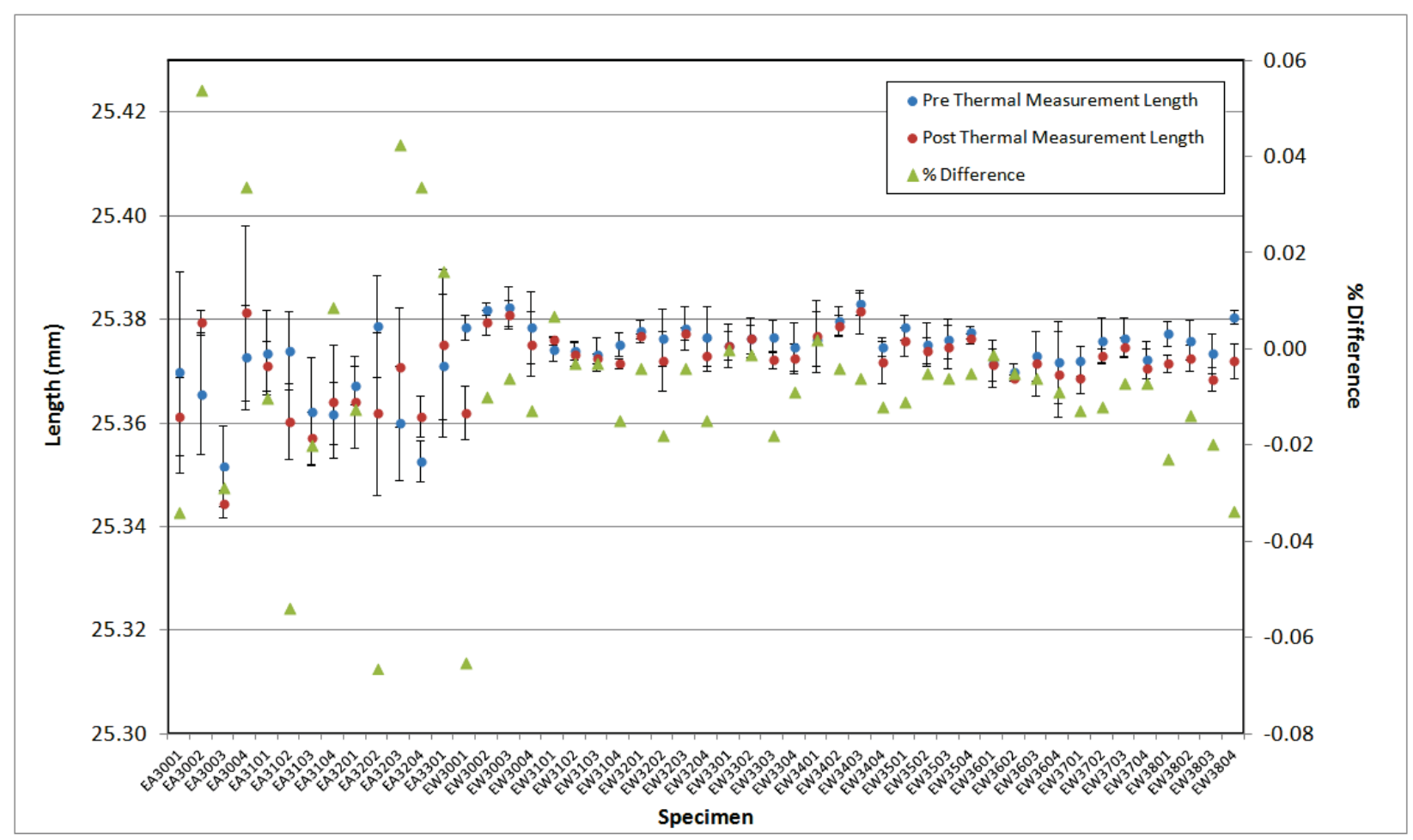

Figure A-82. IG-110 Creep Pre vs. Post Thermal Measurement Length Comparison.

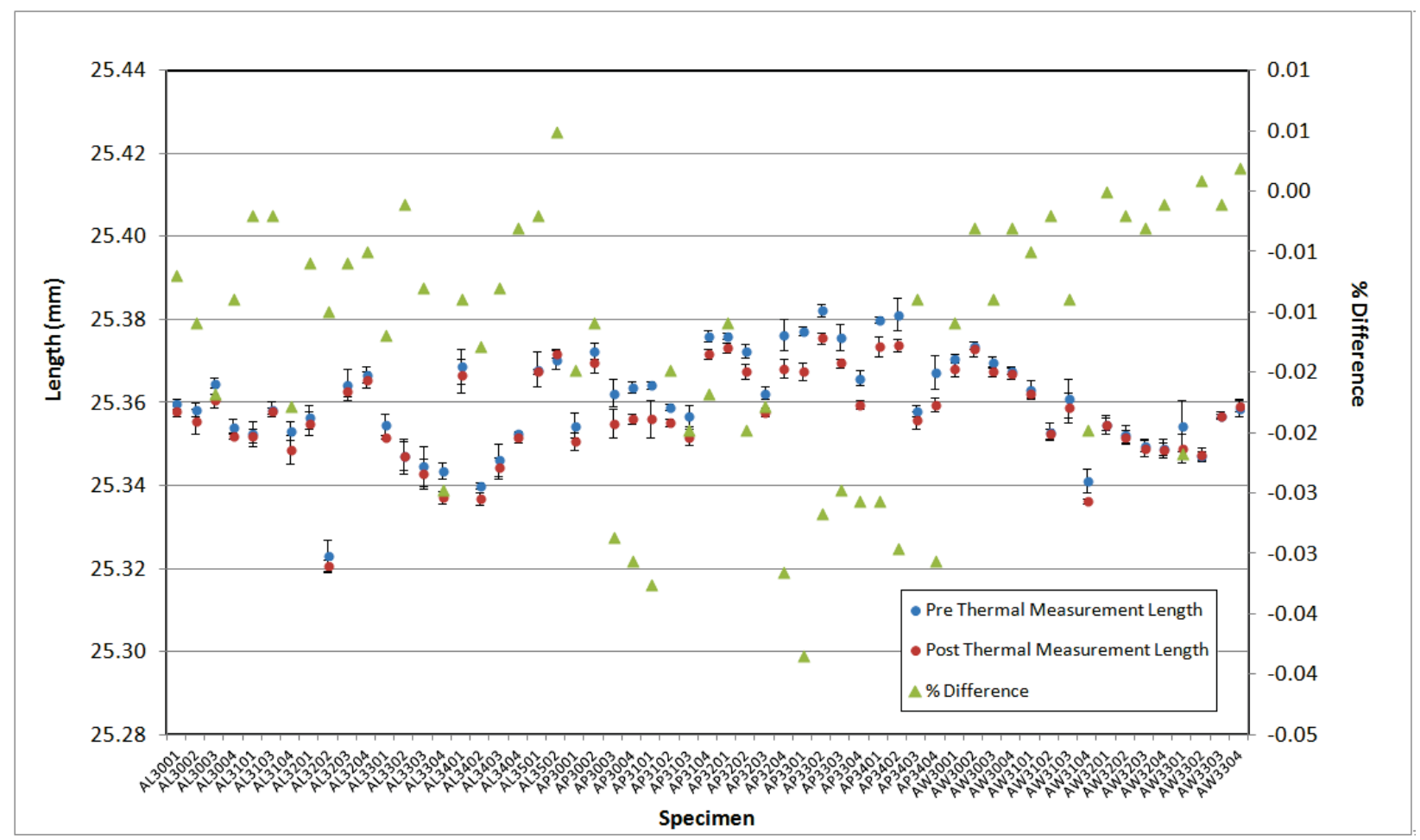

Figure A-83. NBG-17 Creep Pre vs. Post Thermal Measurement Length Comparison. 


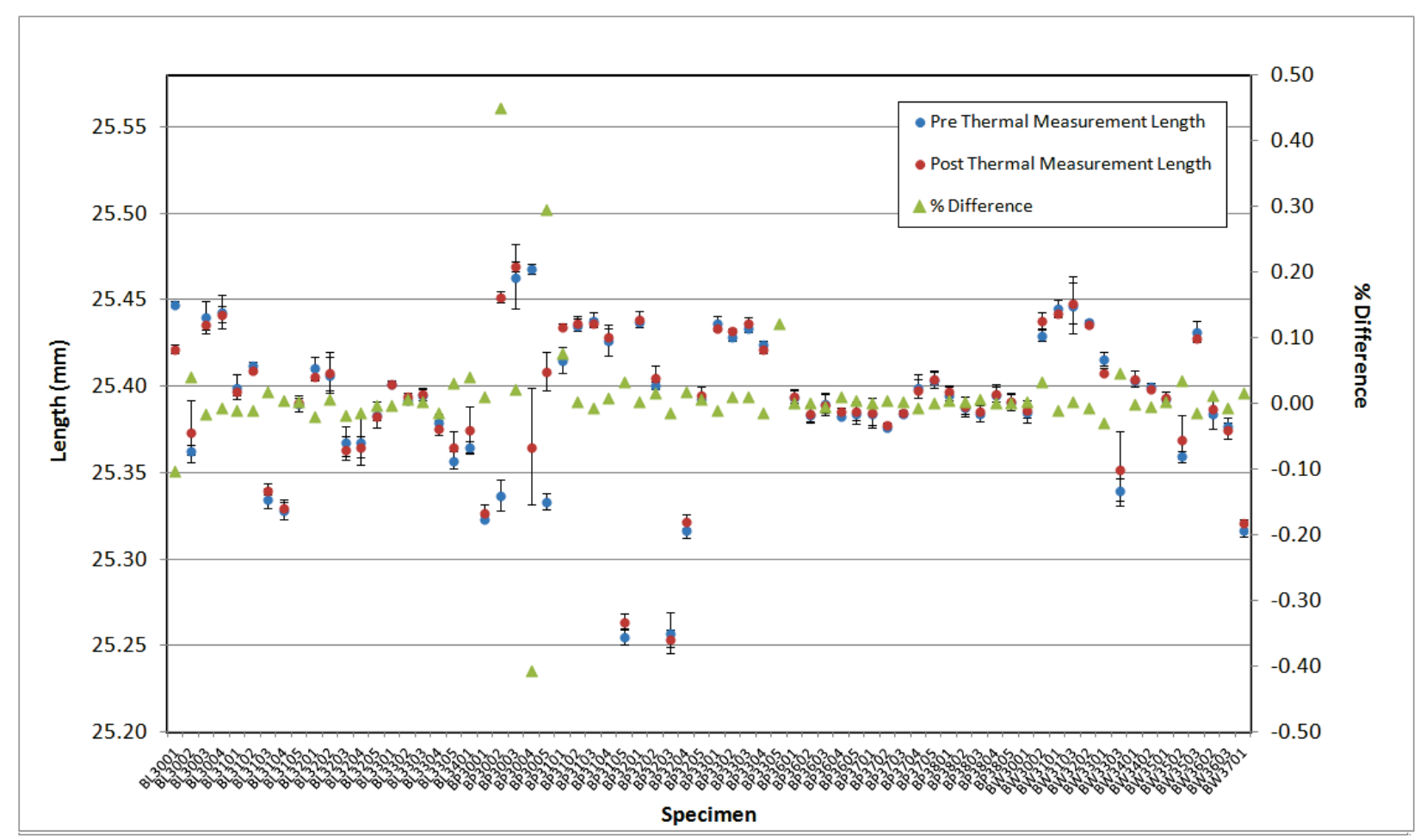

Figure A-84. NBG-18 Creep Pre vs. Post Thermal Measurement Length Comparison.

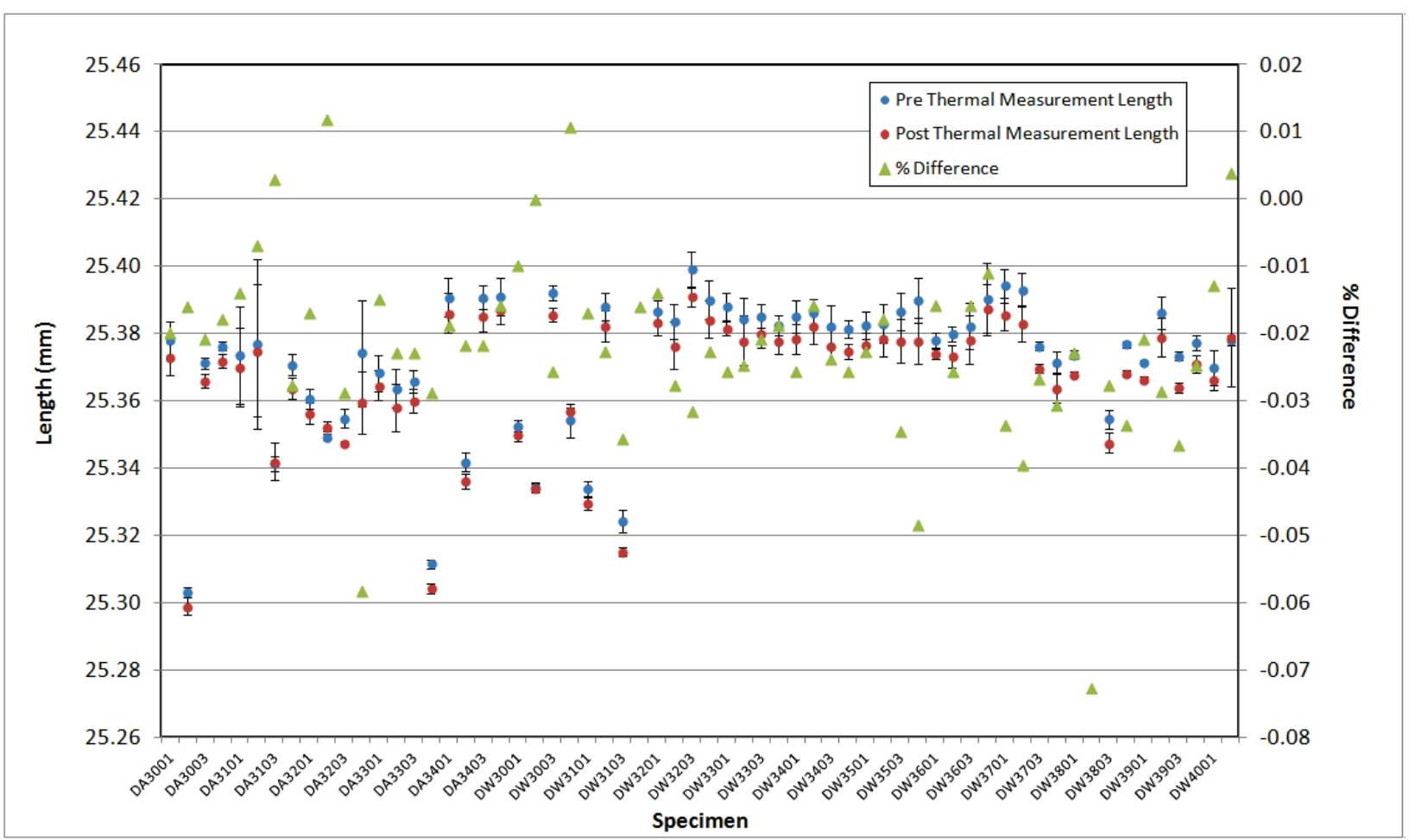

Figure A-85. PCEA Creep Pre vs. Post Thermal Measurement Length Comparison. 


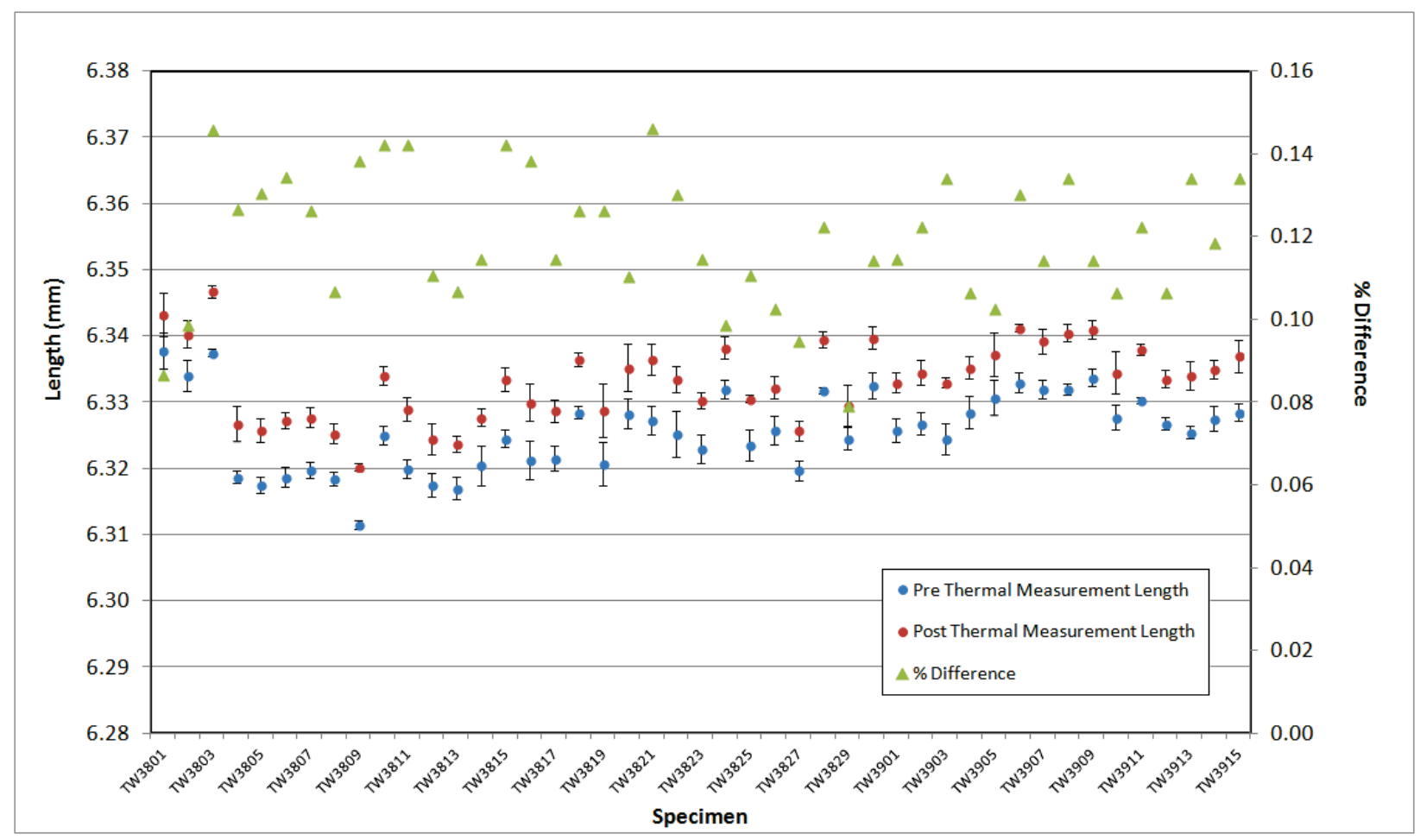

Figure A-86. 2114 Piggyback Pre vs. Post Thermal Measurement Length Comparison.

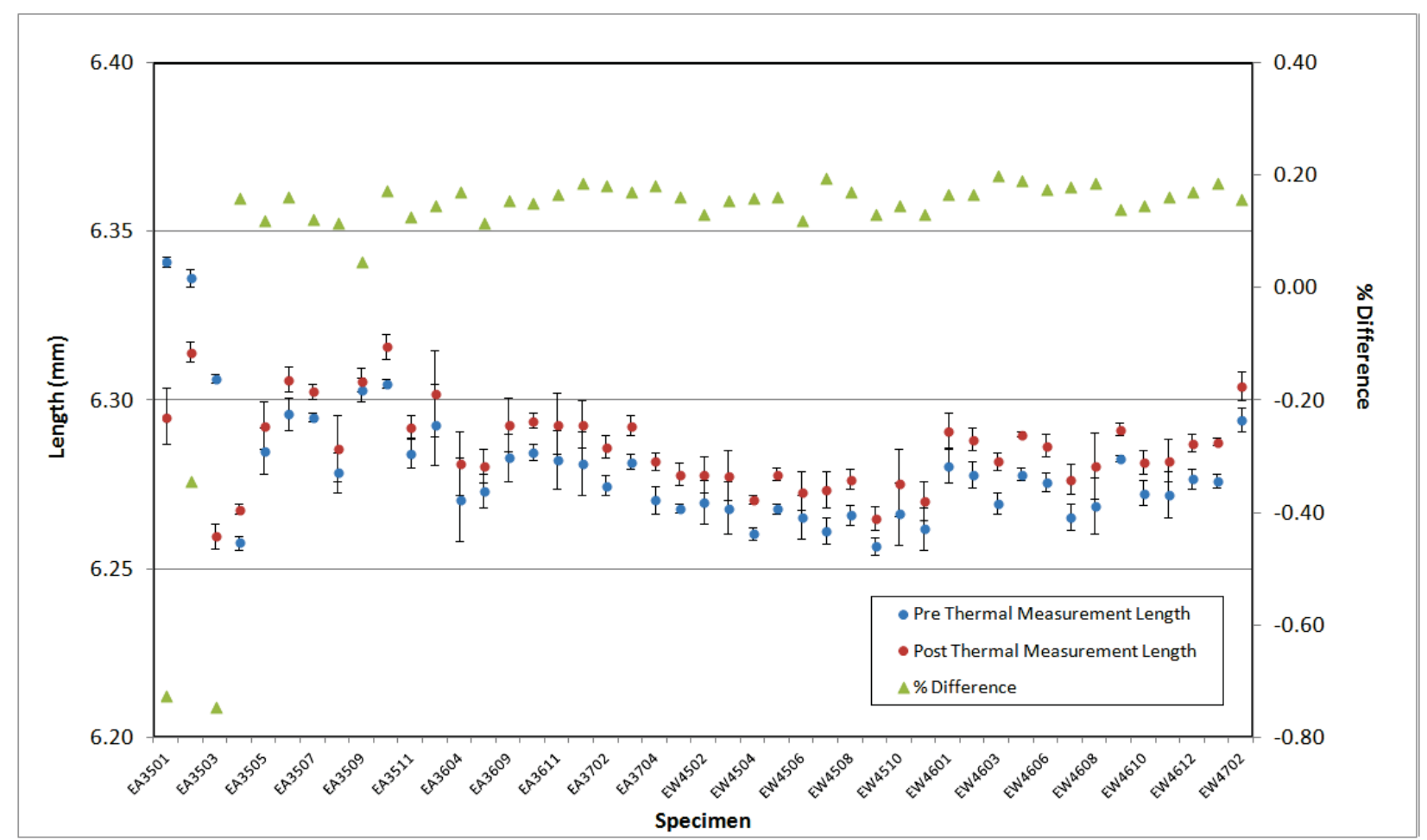

Figure A-87. IG-110 Piggyback Pre vs. Post Thermal Measurement Length Comparison. 


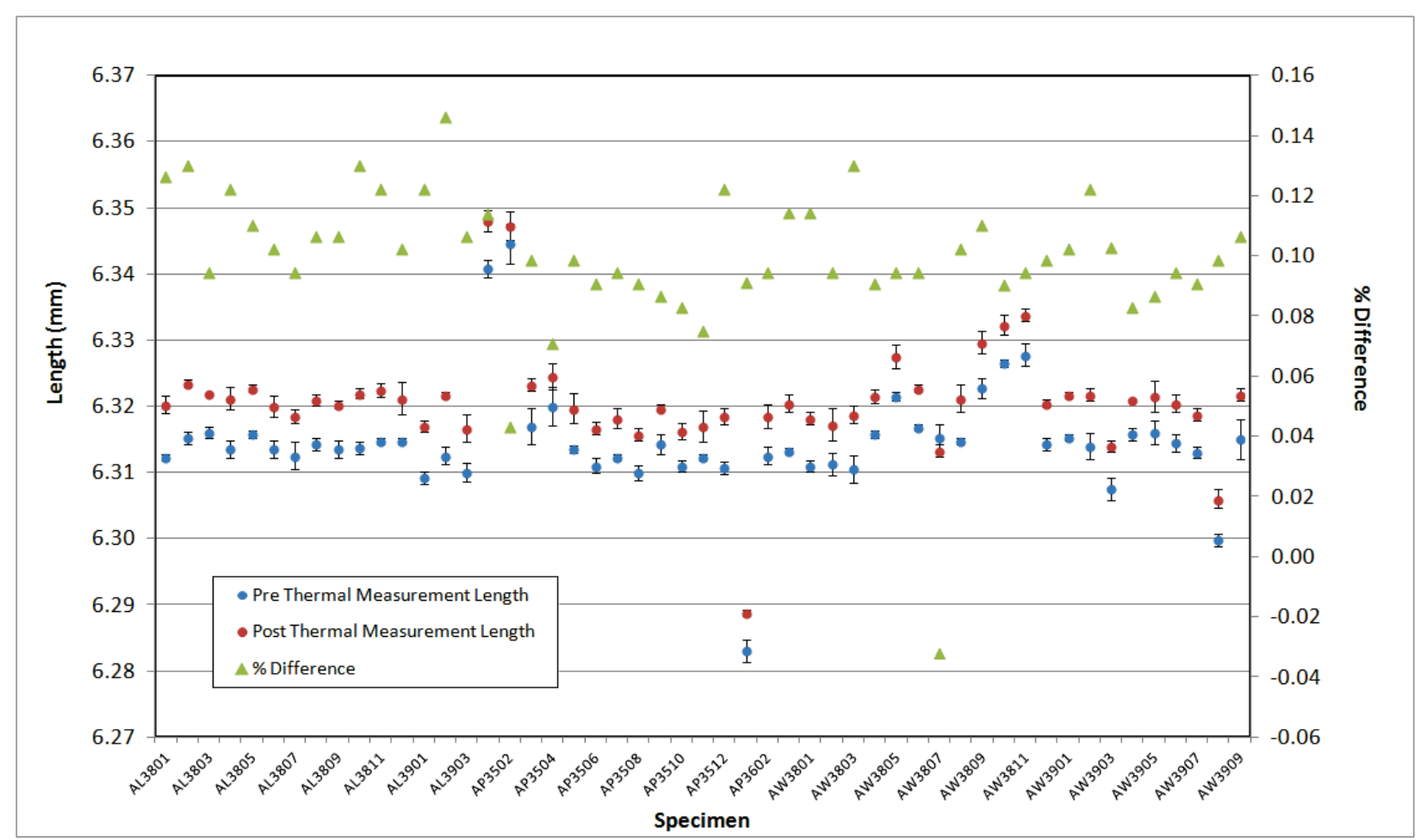

Figure A-88. NBG-17 Piggyback Pre vs. Post Thermal Measurement Length Comparison.

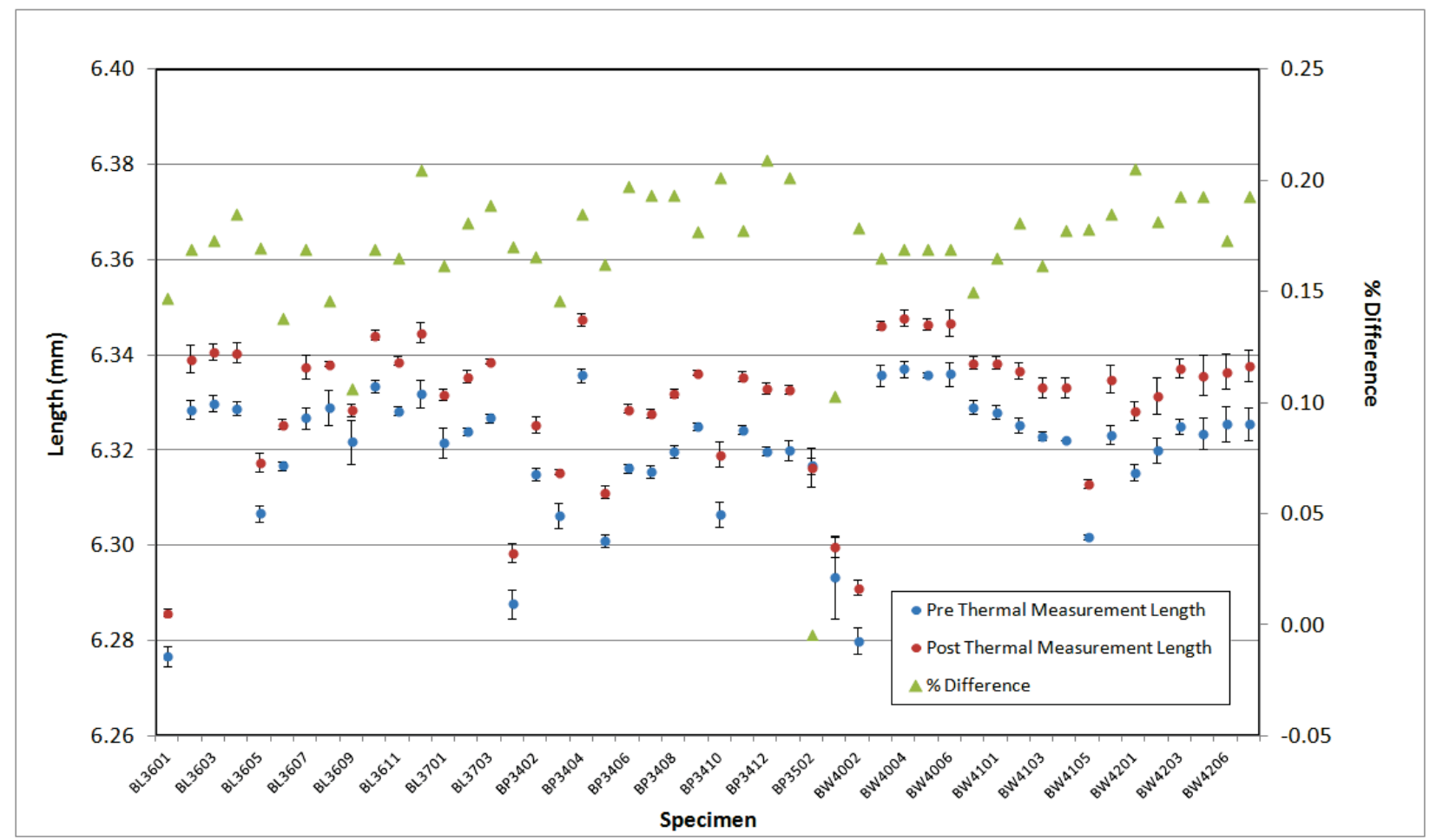

Figure A-89. NBG-18 Piggyback Pre vs. Post Thermal Measurement Length Comparison. 


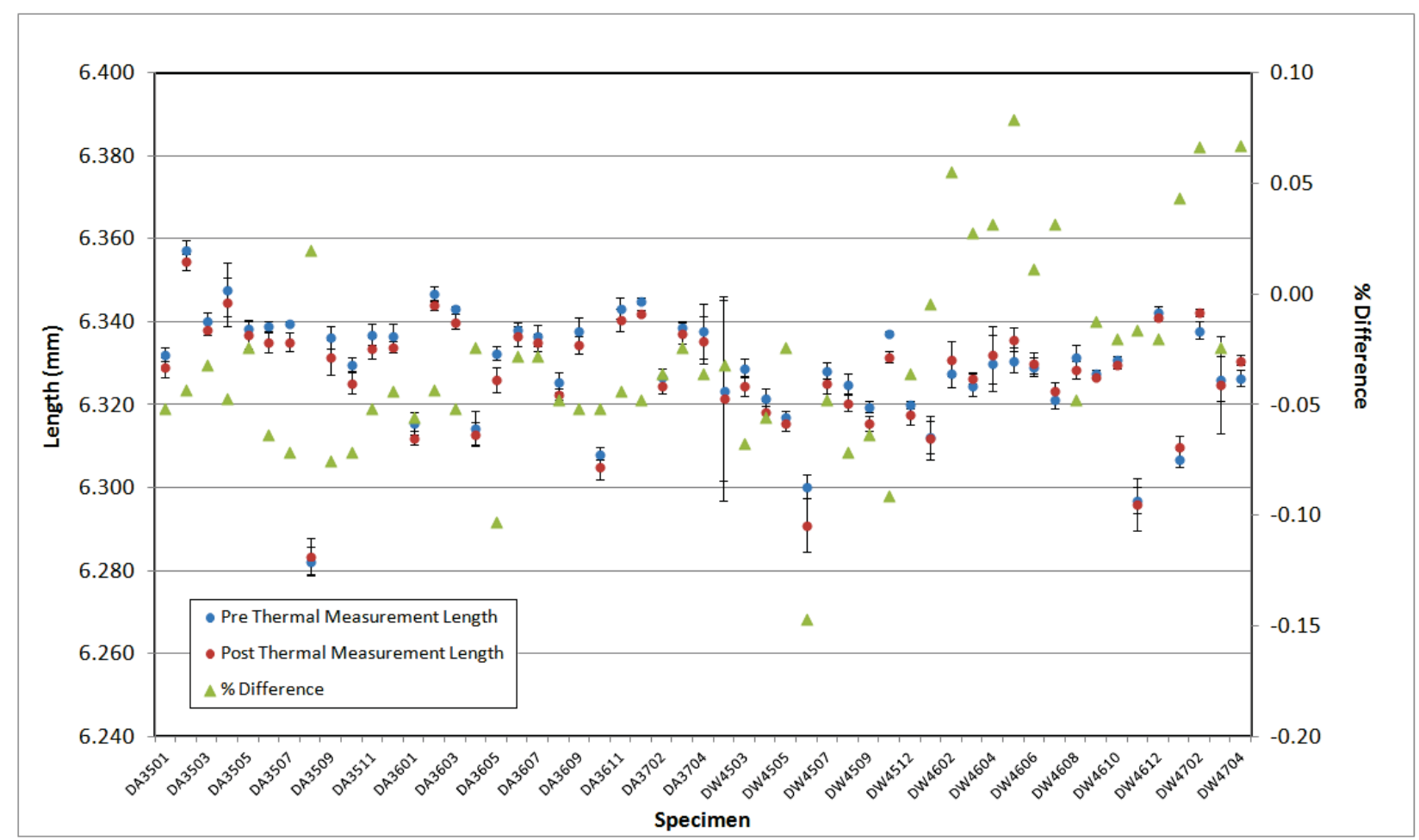

Figure A-90. PCEA Piggyback Pre vs. Post Thermal Measurement Length Comparison.

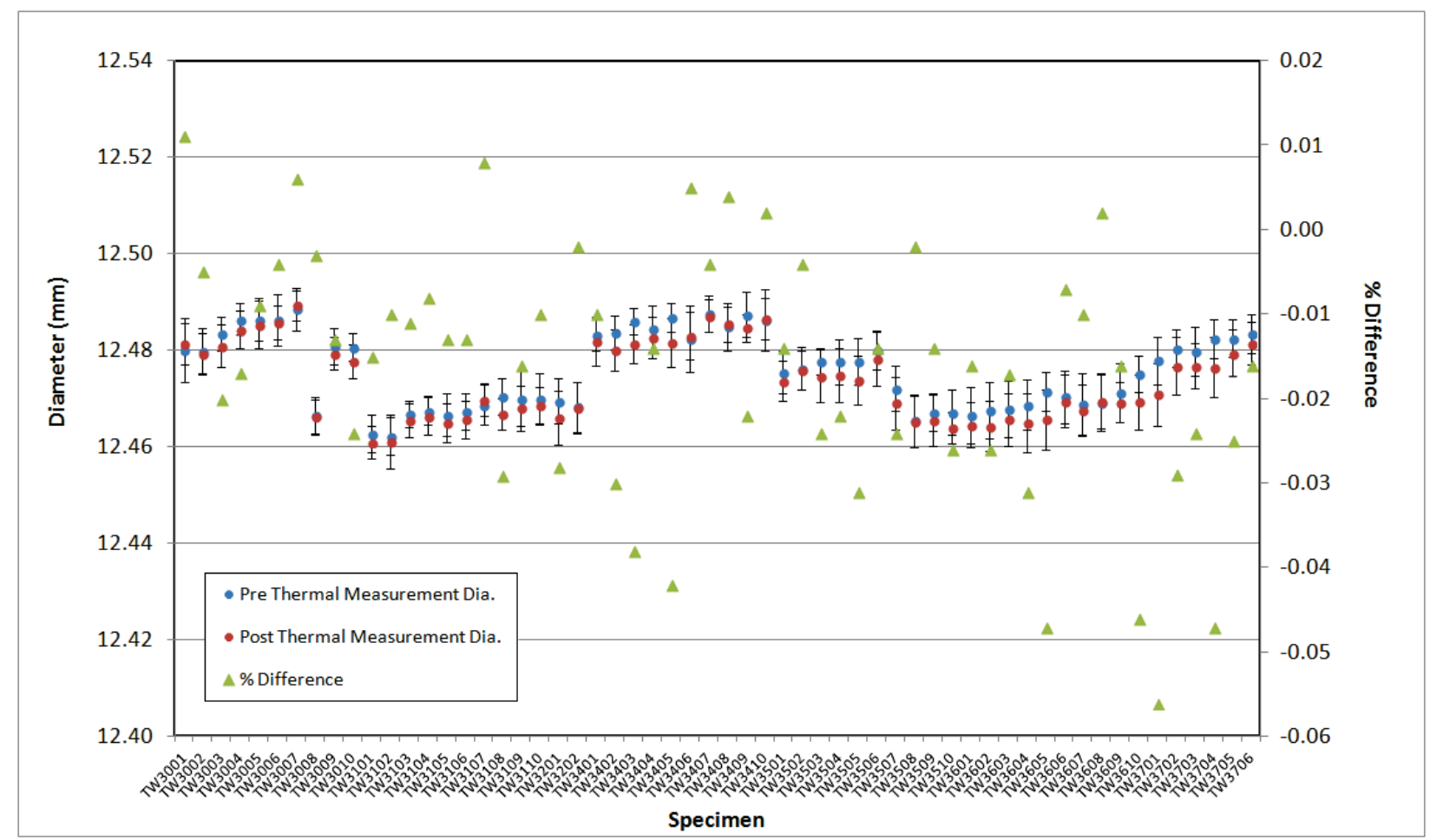

Figure A-91. 2114 Creep Pre vs. Post Thermal Measurement Diameter Comparison. 


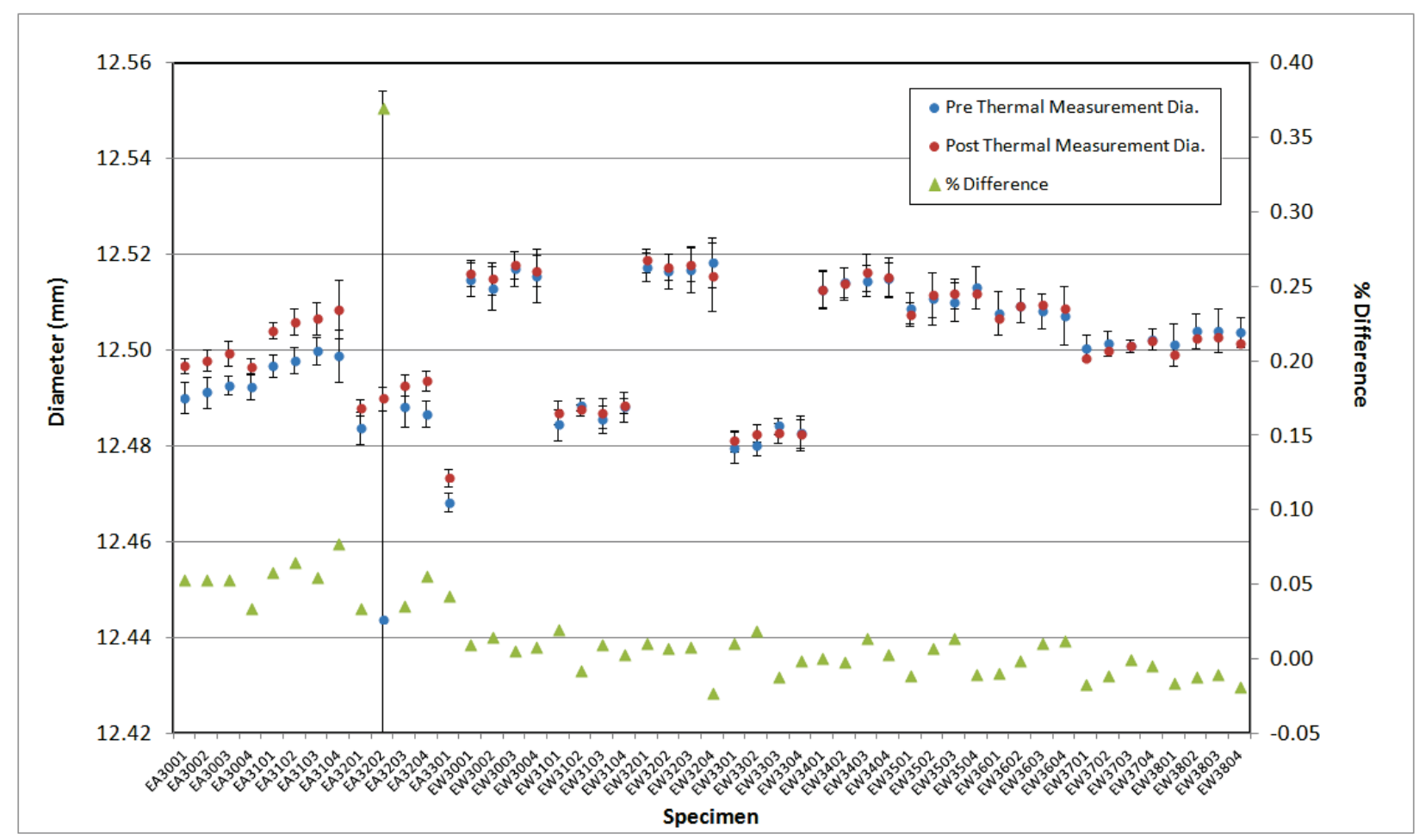

Figure A-92. IG-110 Creep Pre vs. Post Thermal Measurement Diameter Comparison.

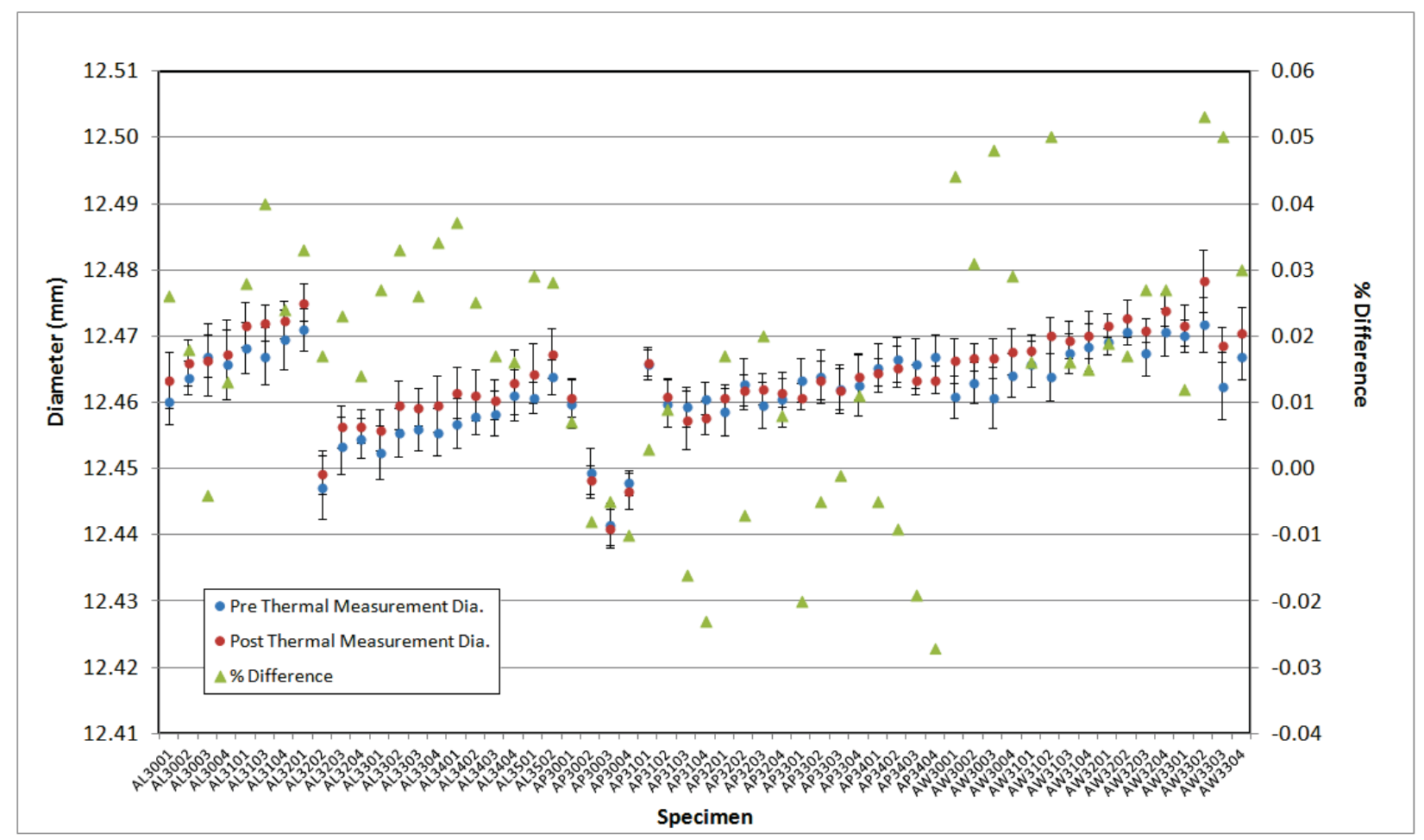

Figure A-93. NBG-17 Creep Pre vs. Post Thermal Measurement Diameter Comparison. 


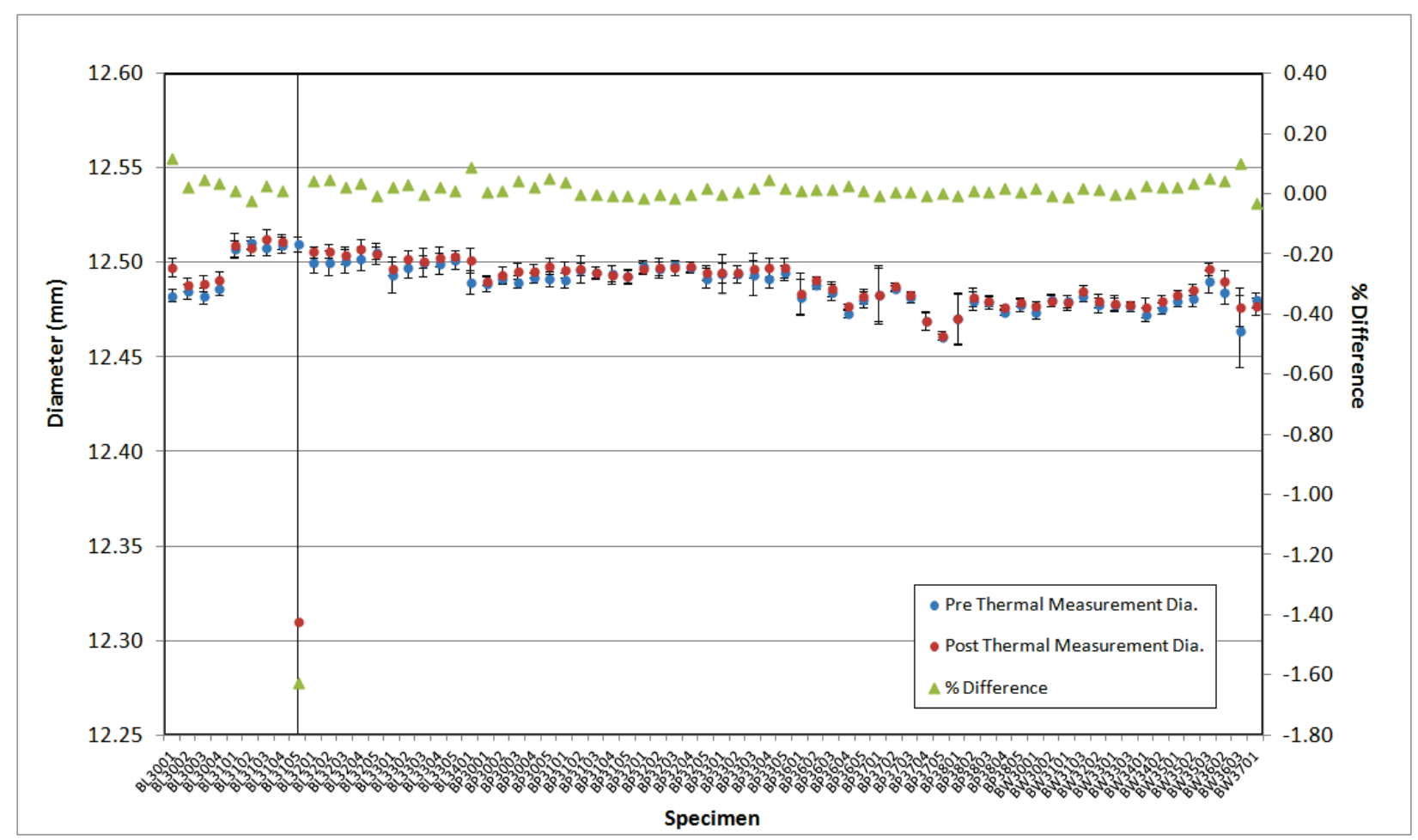

Figure A-94. NBG-18 Creep Pre vs. Post Thermal Measurement Diameter Comparison.

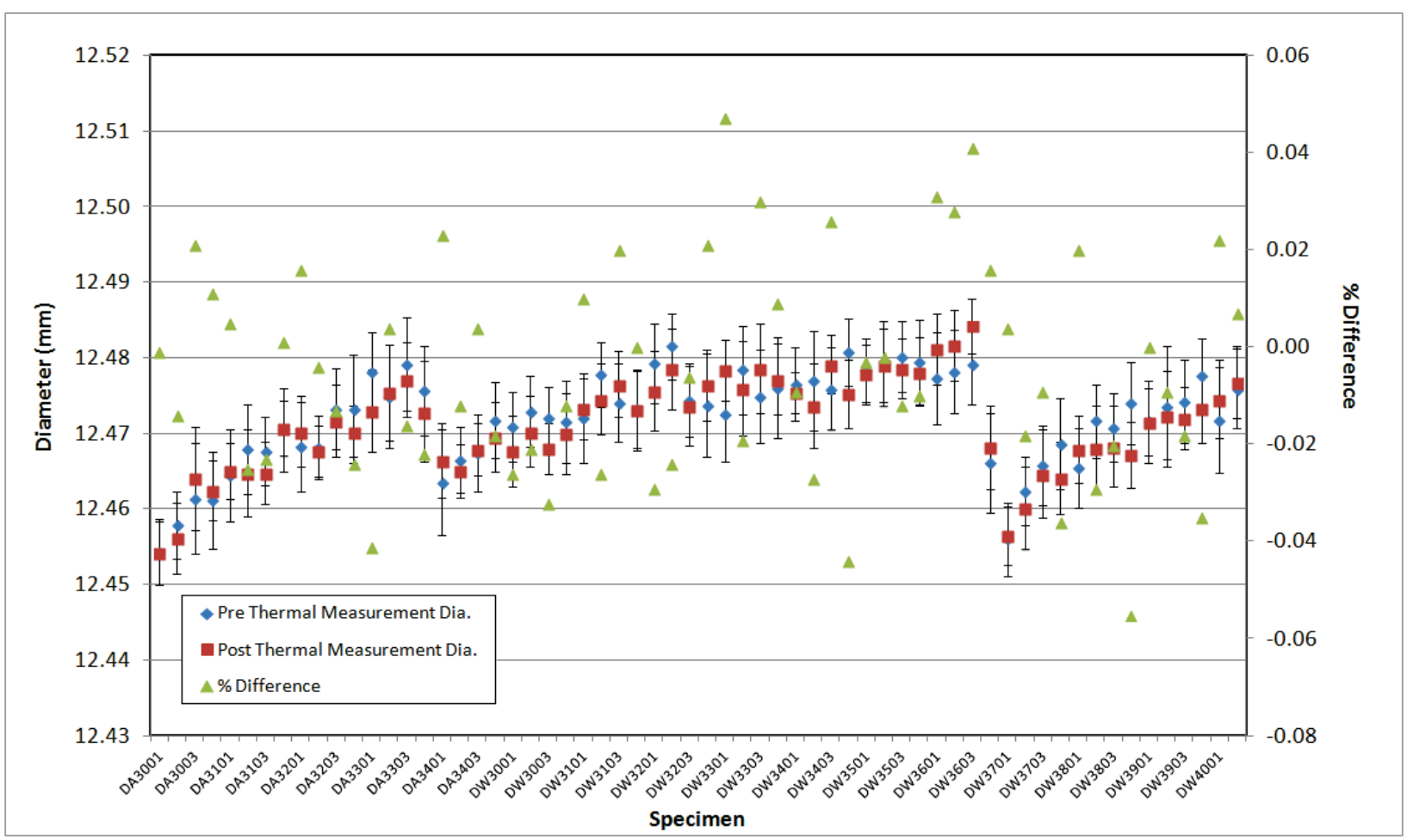

Figure A-95. PCEA Creep Pre vs. Post Thermal Measurement Diameter Comparison. 


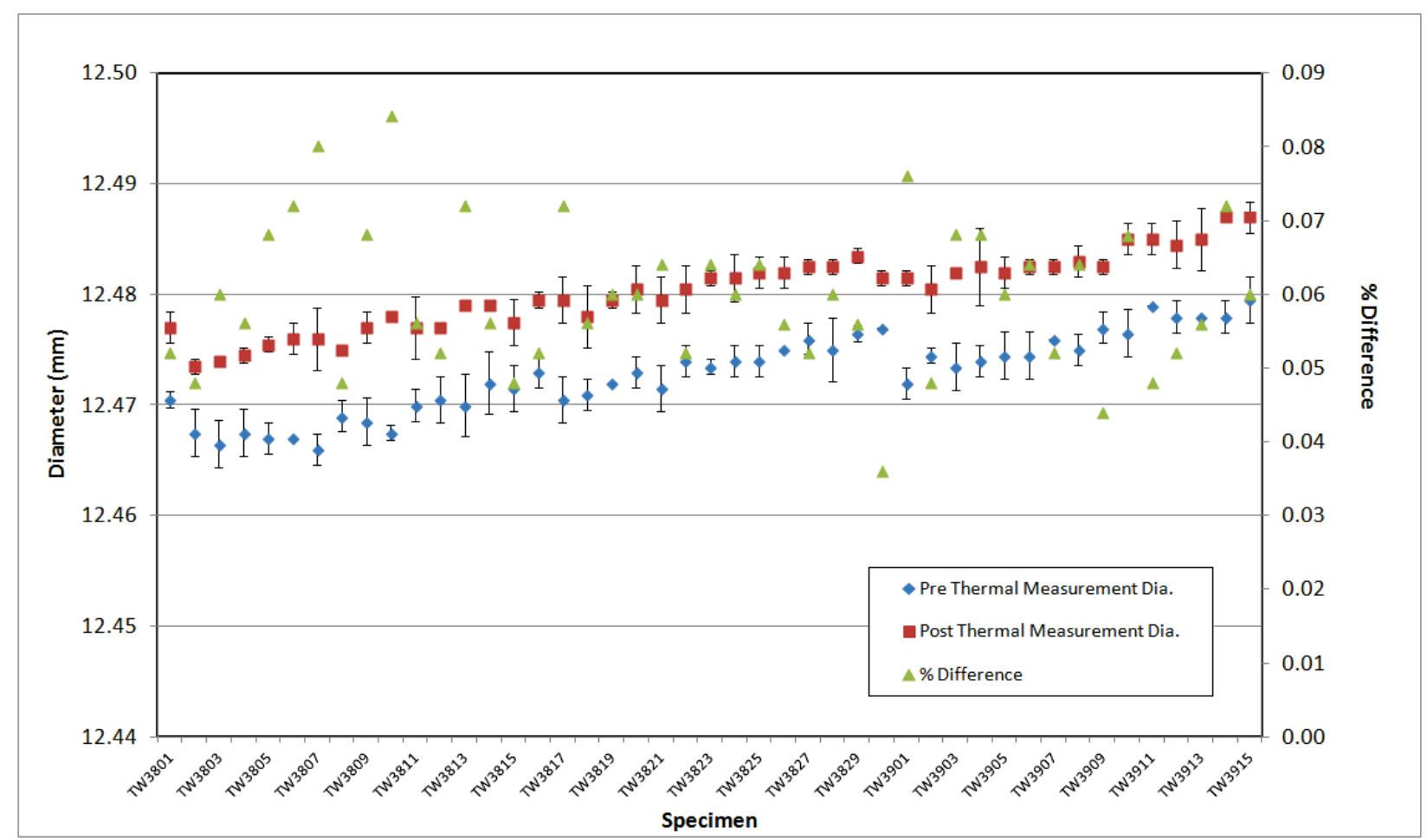

Figure A-96. 2114 Piggyback Pre vs. Post Thermal Measurement Diameter Comparison.

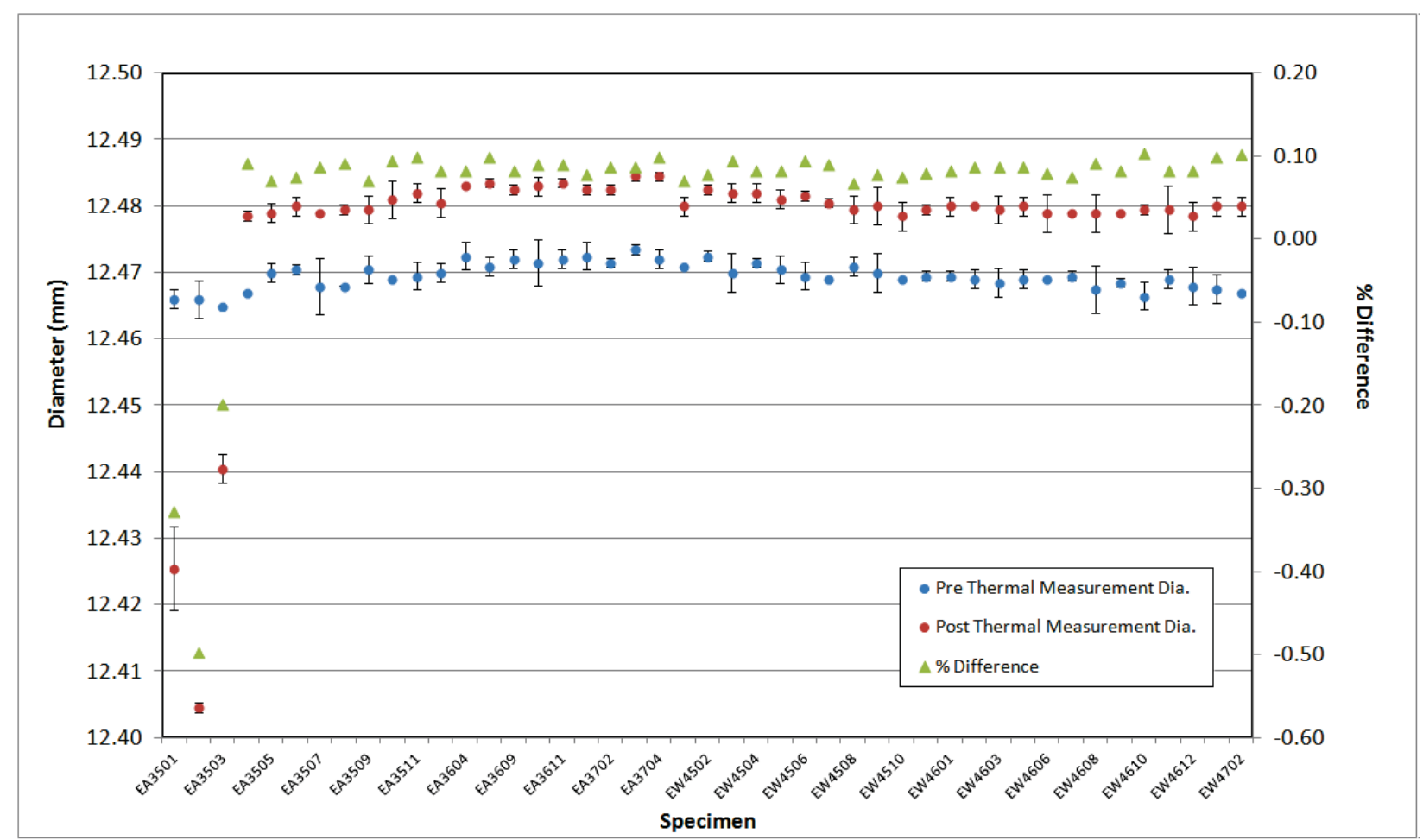

Figure A-97. IG-110 Piggyback Pre vs. Post Thermal Measurement Diameter Comparison. 


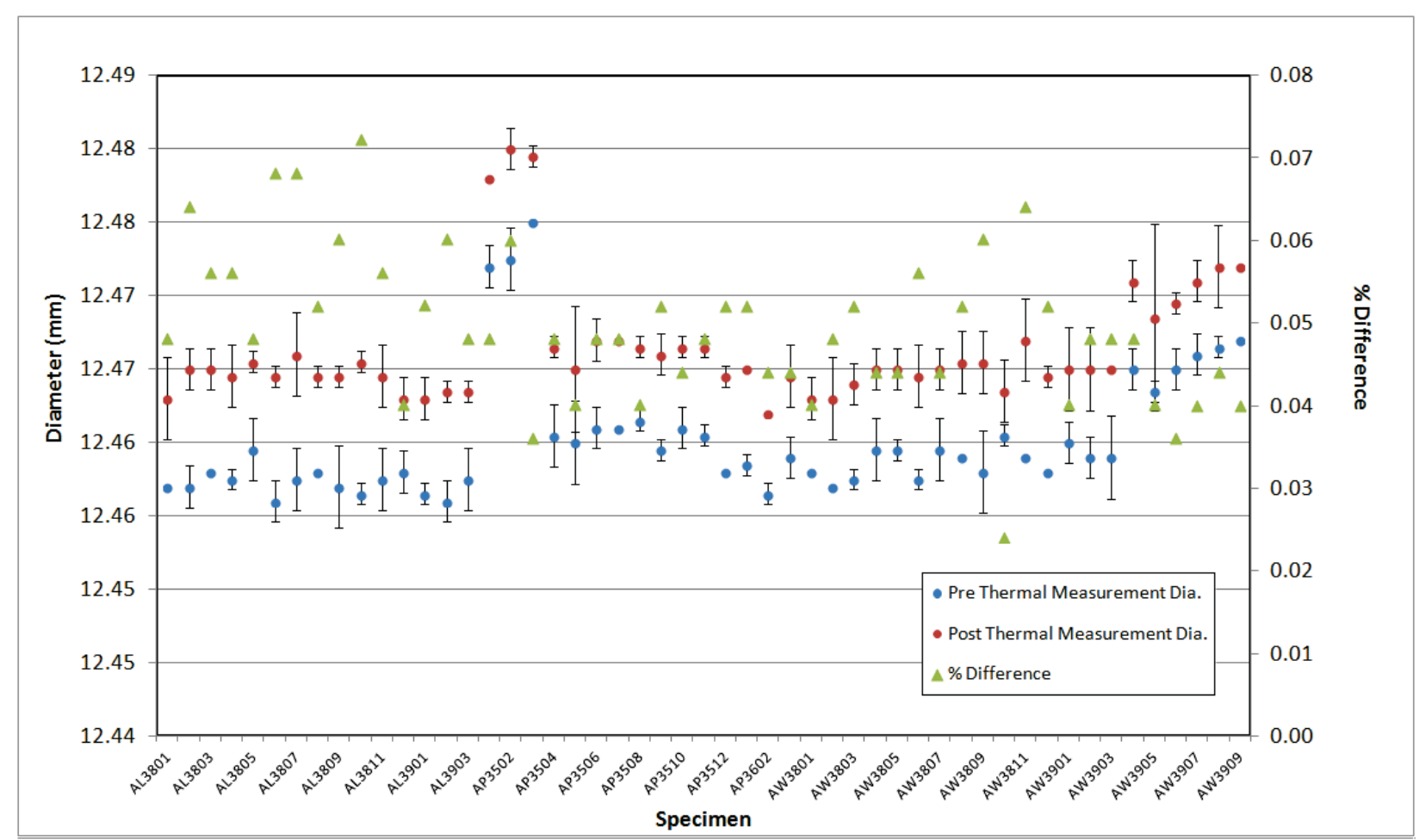

Figure A-98. NBG-17 Piggyback Pre vs. Post Thermal Measurement Diameter Comparison.

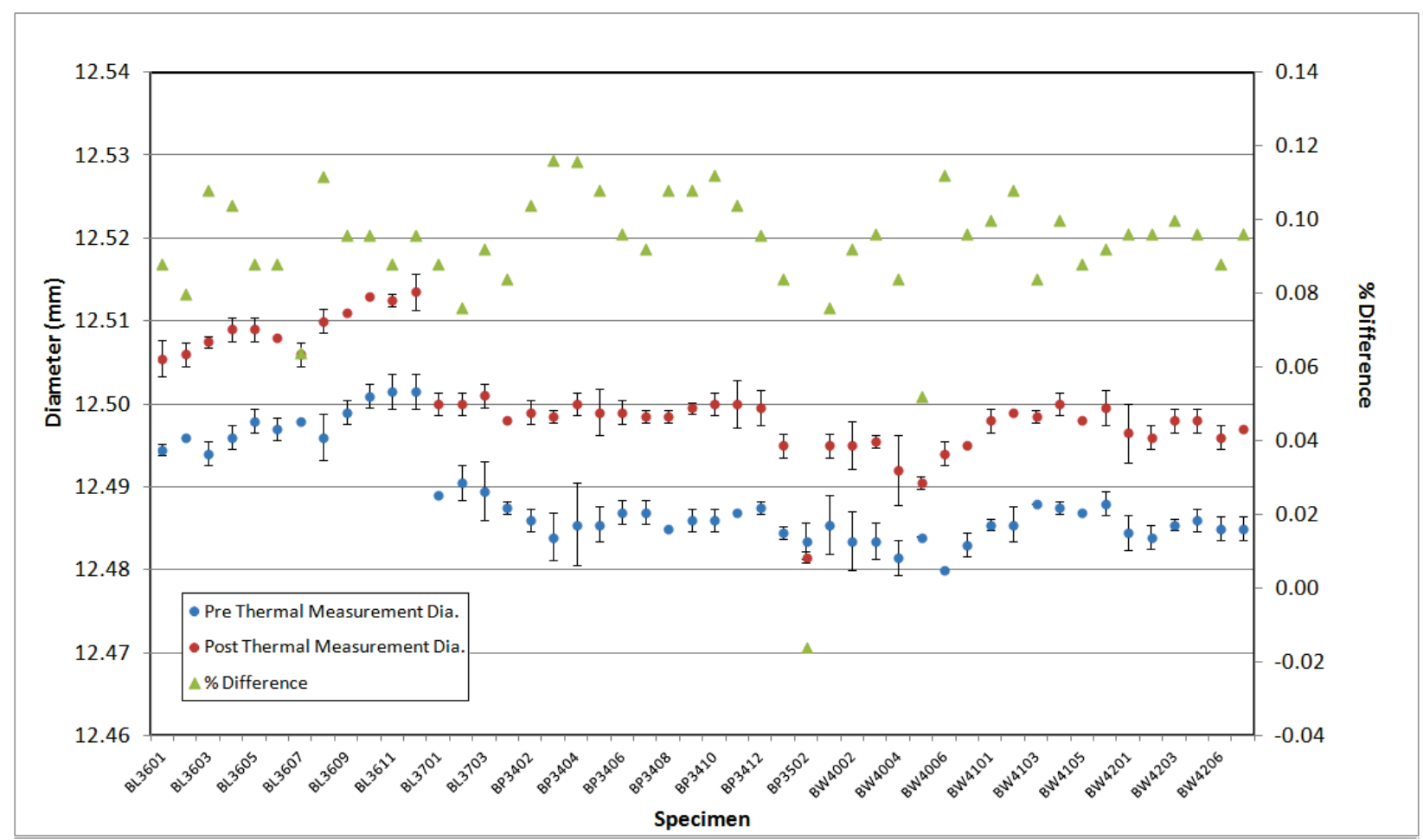

Figure A-99. NBG-18 Piggyback Pre vs. Post Thermal Measurement Diameter Comparison. 


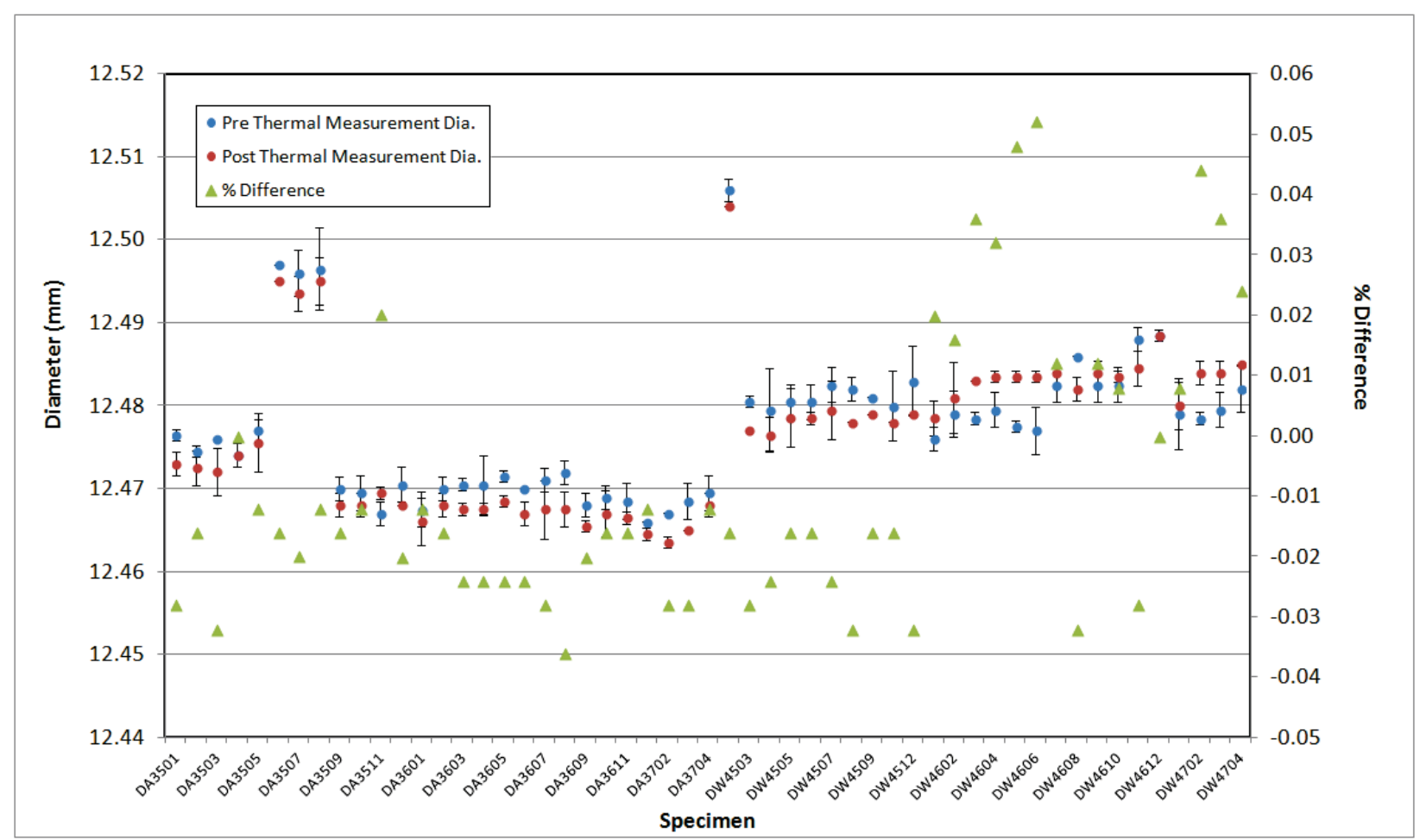

Figure A-100. PCEA Piggyback Pre vs. Post Thermal Measurement Diameter Comparison.

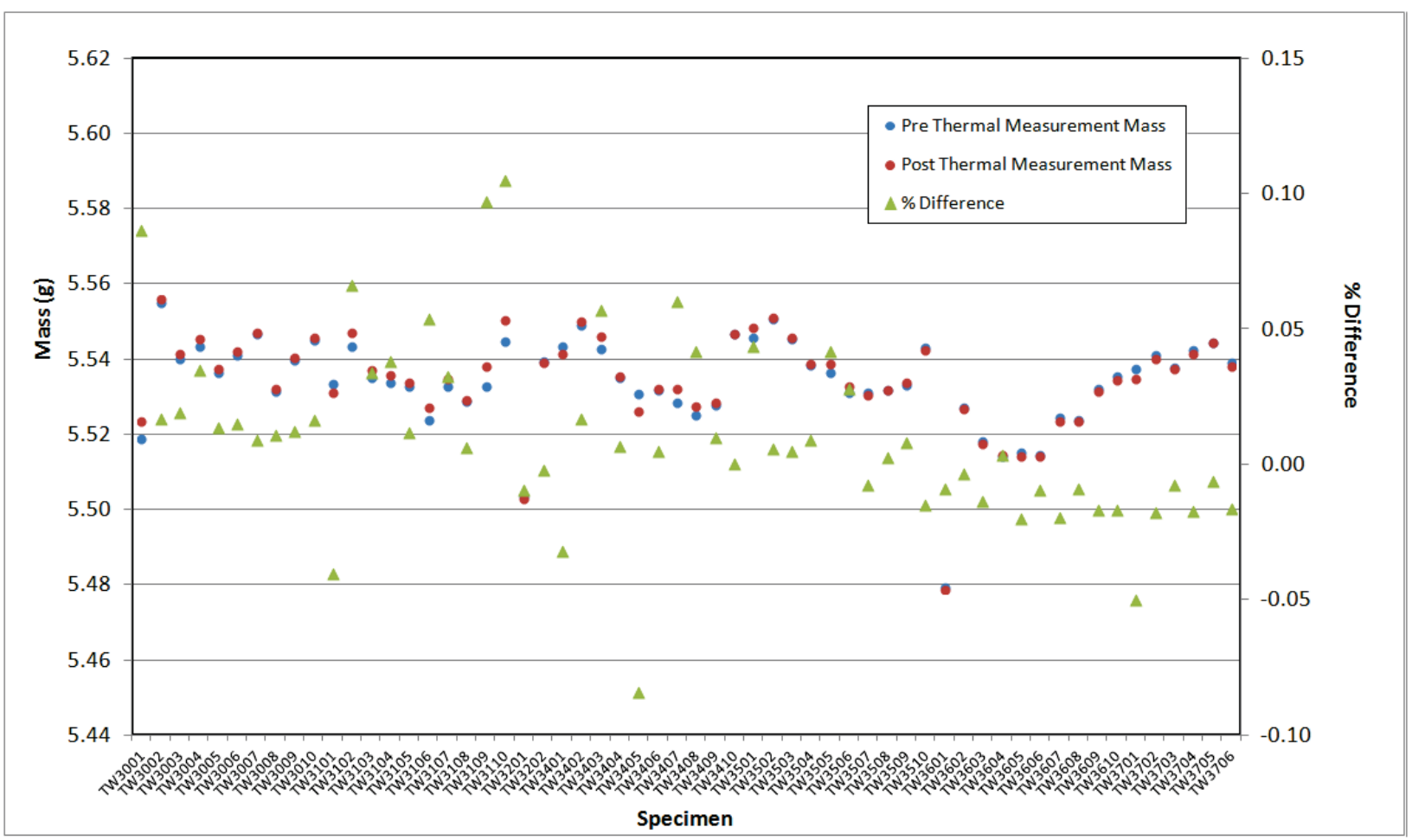

Figure A-101. 2114 Creep Pre vs. Post Thermal Measurement Mass Comparison. 


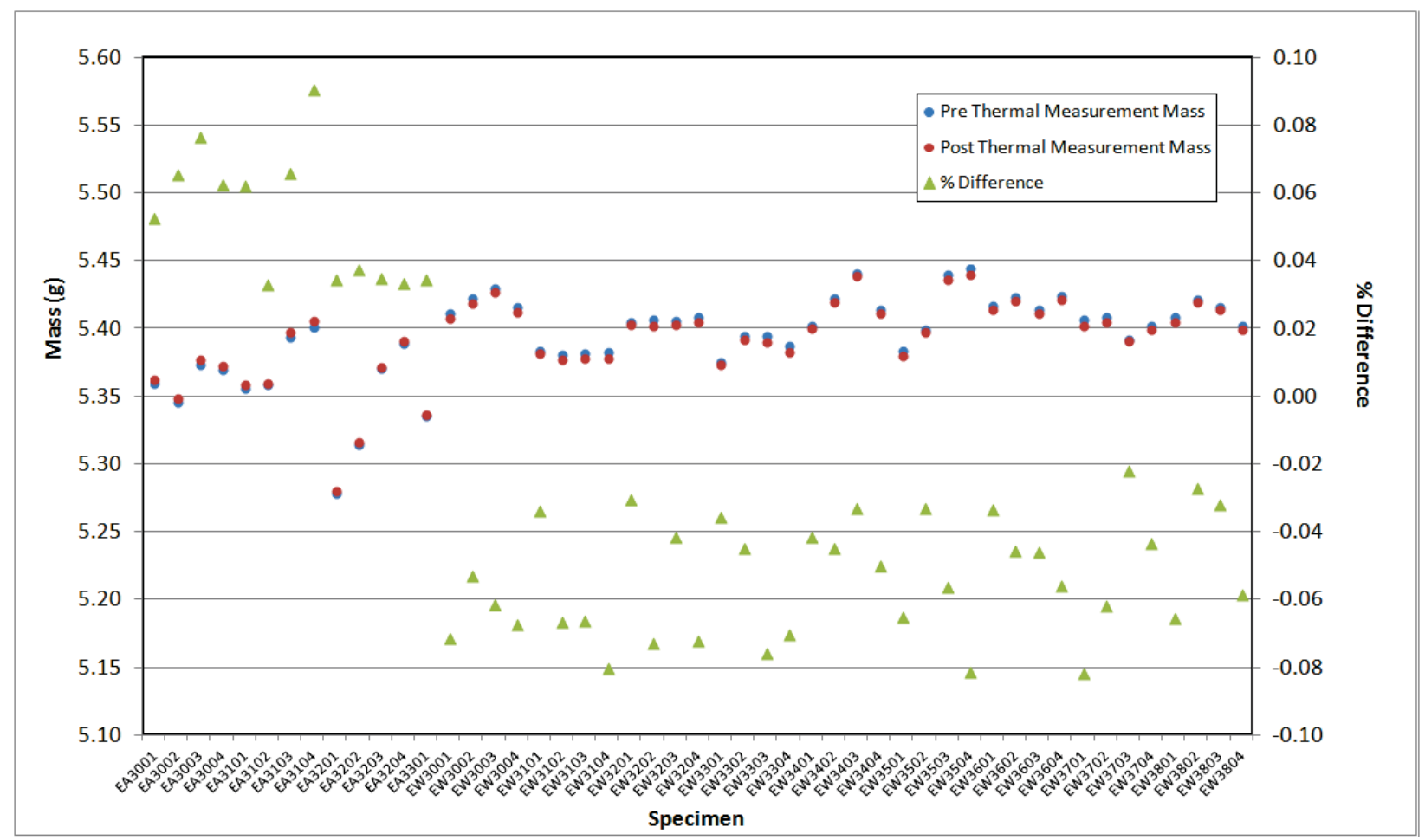

Figure A-102. IG-110 Creep Pre vs. Post Thermal Measurement Mass Comparison.

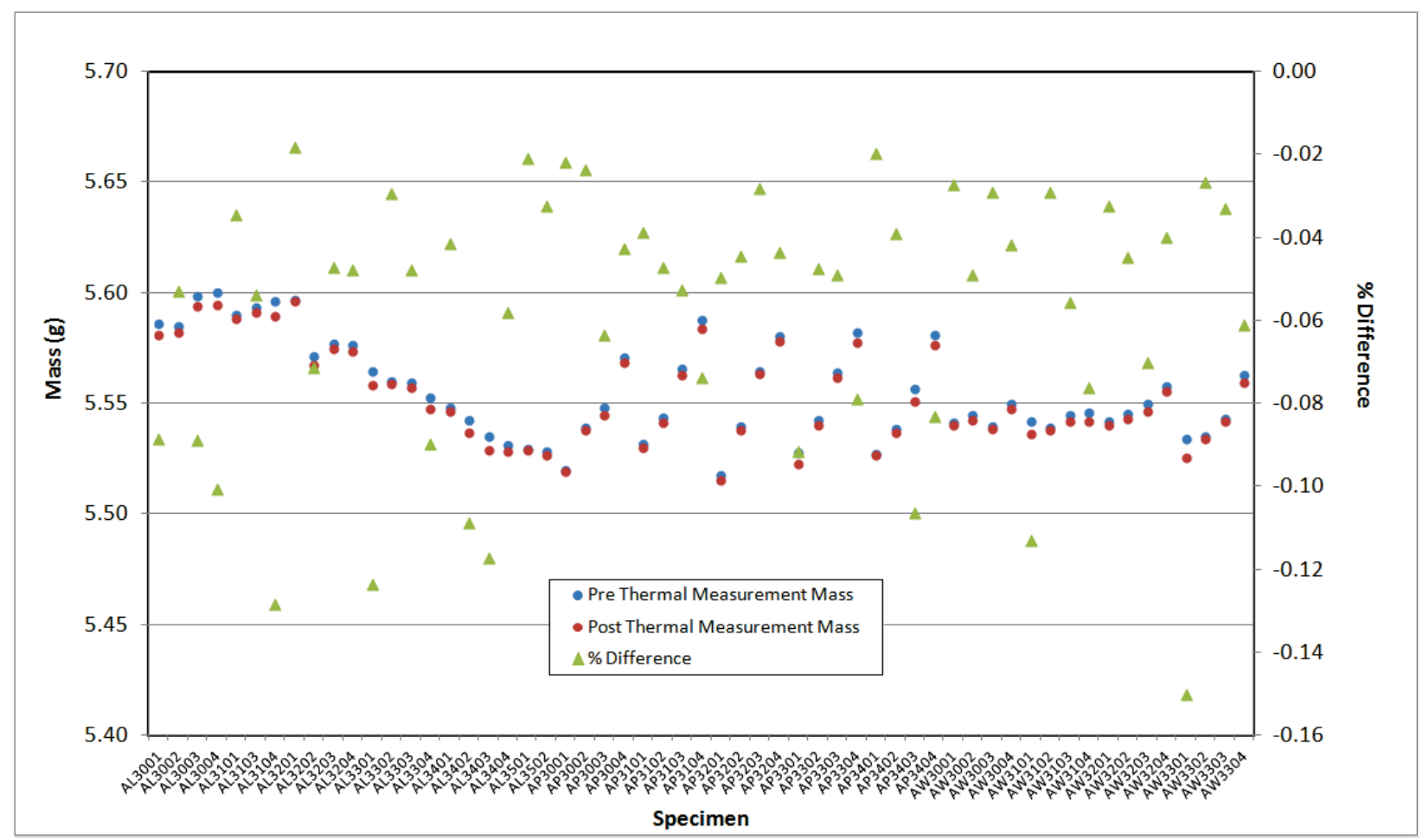

Figure A-103. NBG-17 Creep Pre vs. Post Thermal Measurement Mass Comparison. 


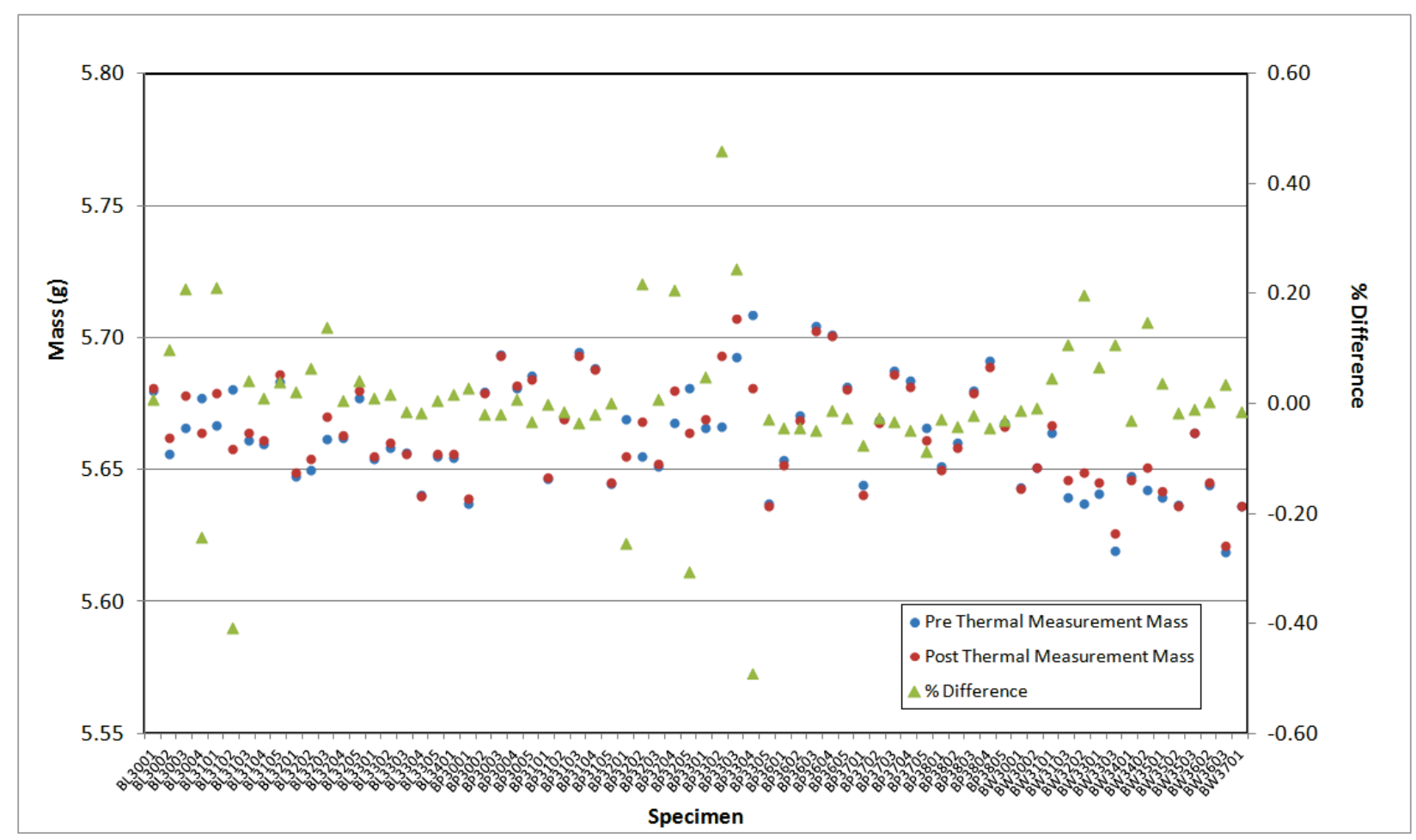

Figure A-104. NBG-18 Creep Pre vs. Post Thermal Measurement Mass Comparison.

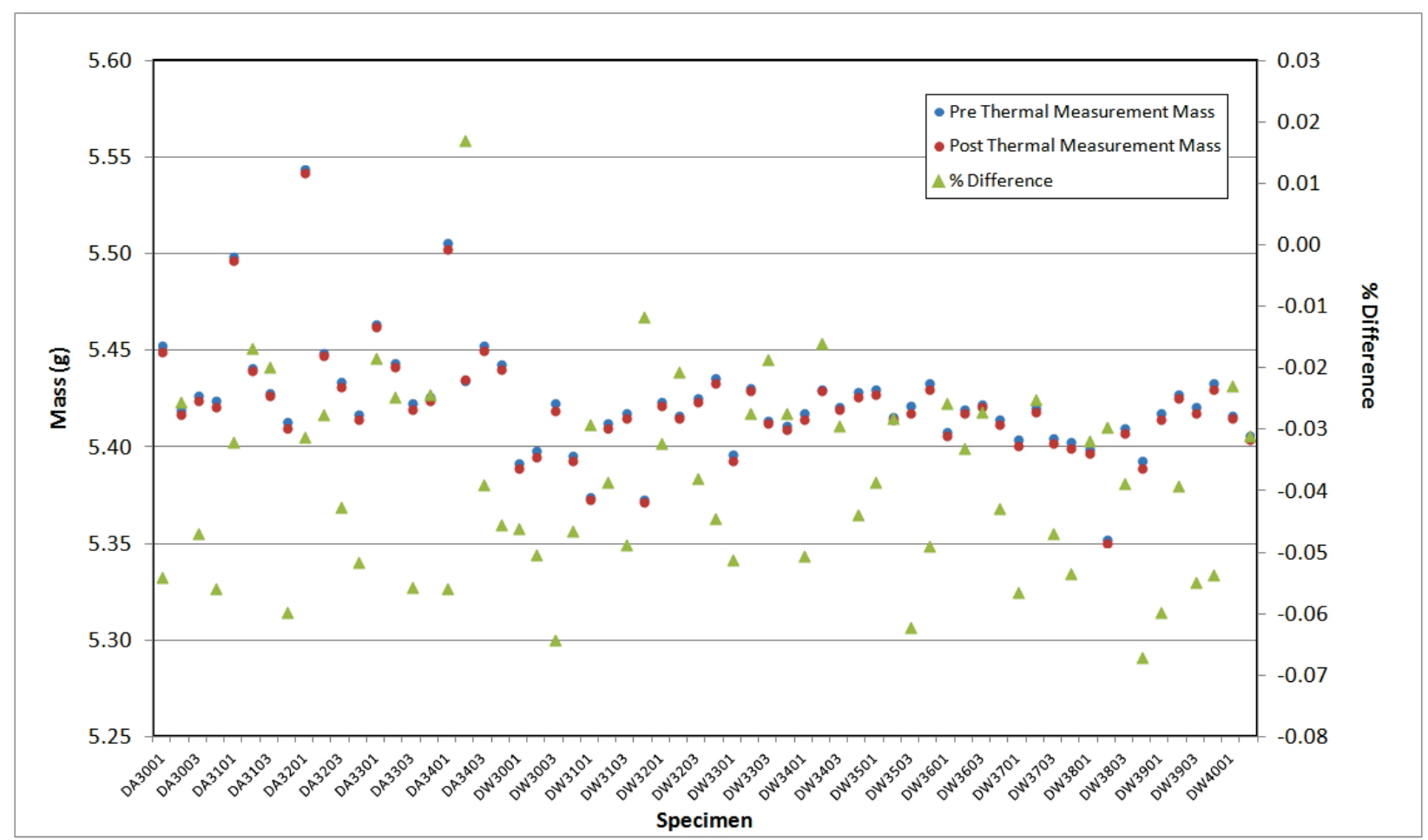

Figure A-105. PCEA Creep Pre vs. Post Thermal Measurement Mass Comparison. 


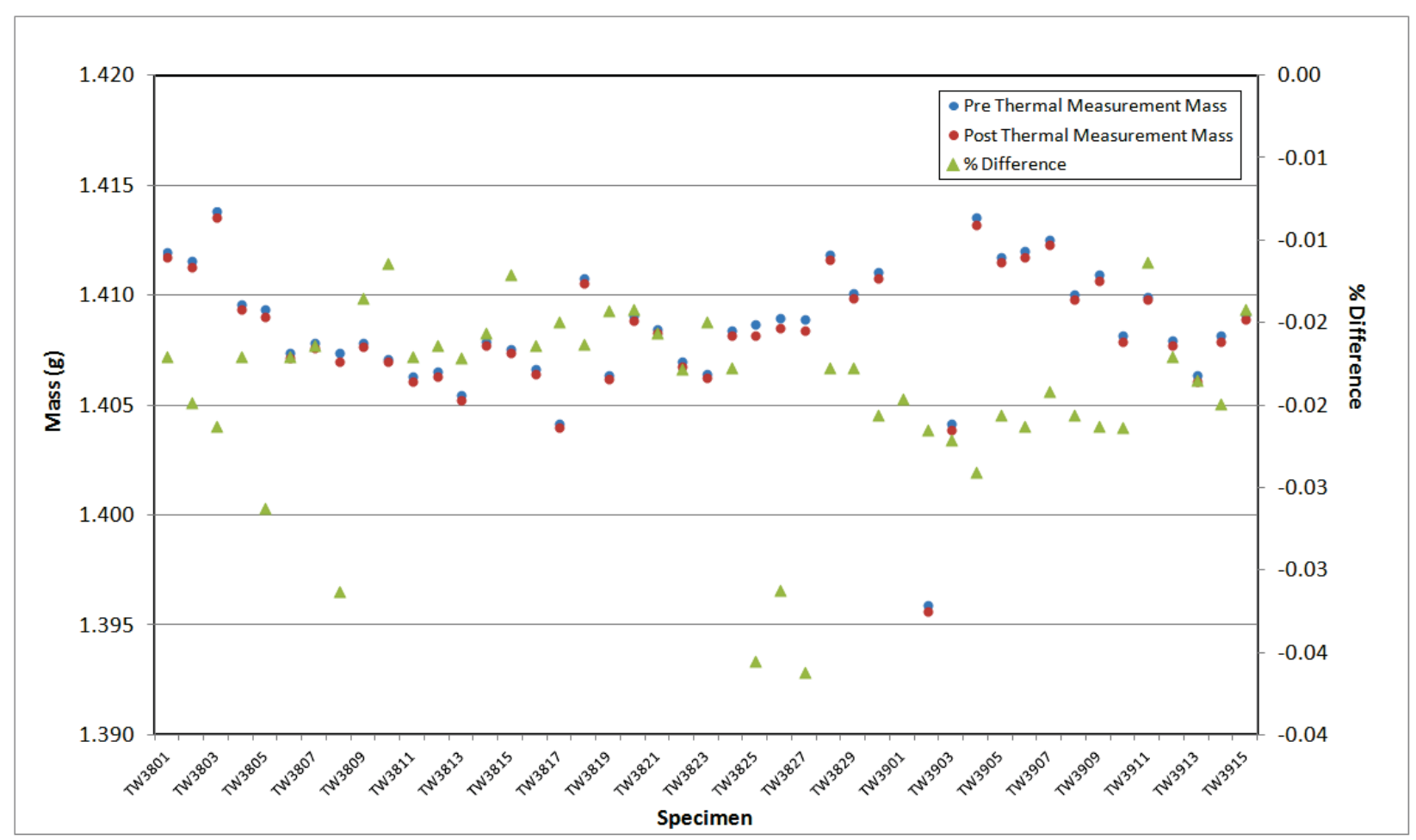

Figure A-106. 2114 Piggyback Pre vs. Post Thermal Measurement Mass Comparison.

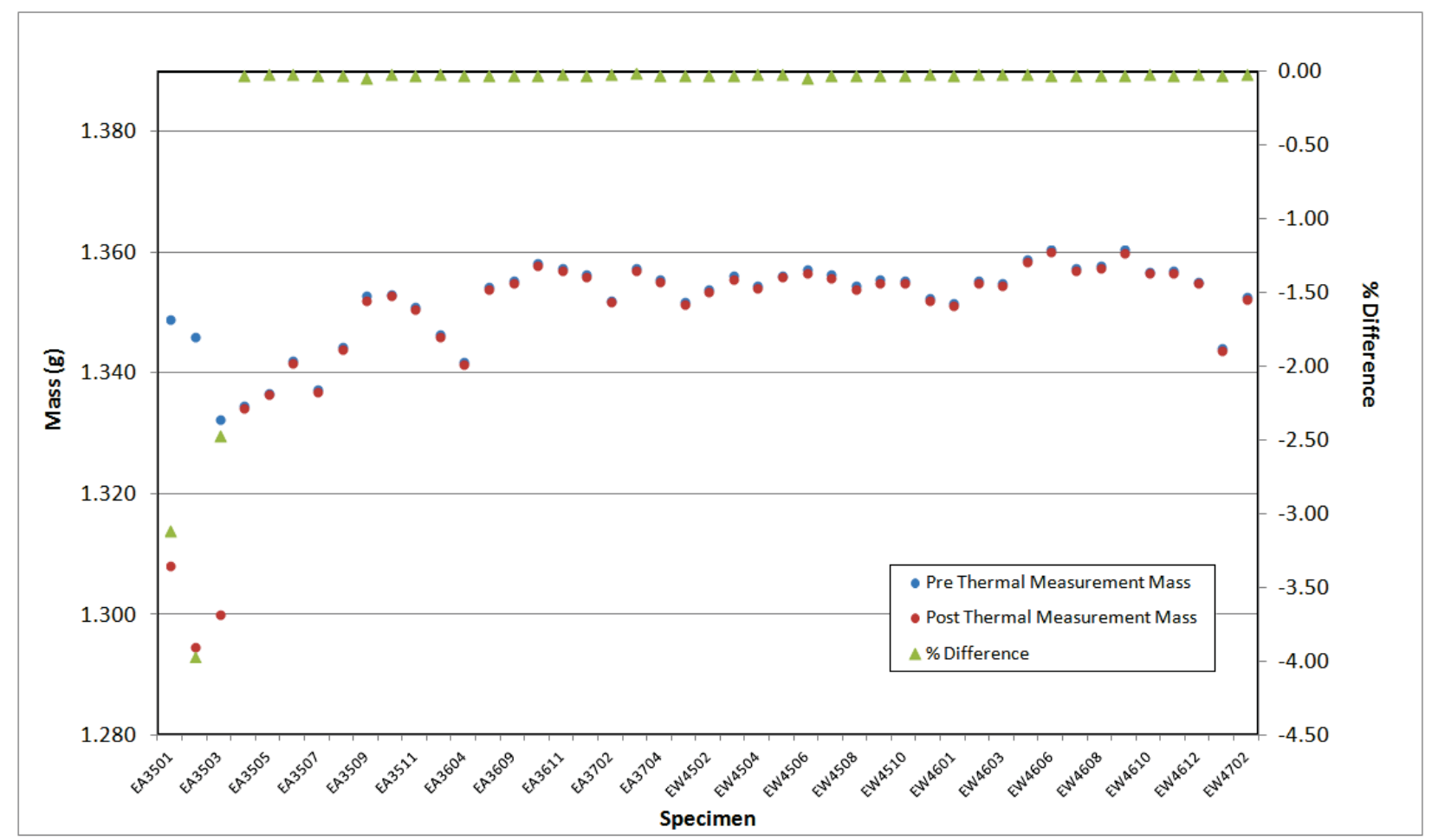

Figure A-107. IG-110 Piggyback Pre vs. Post Thermal Measurement Mass Comparison. 


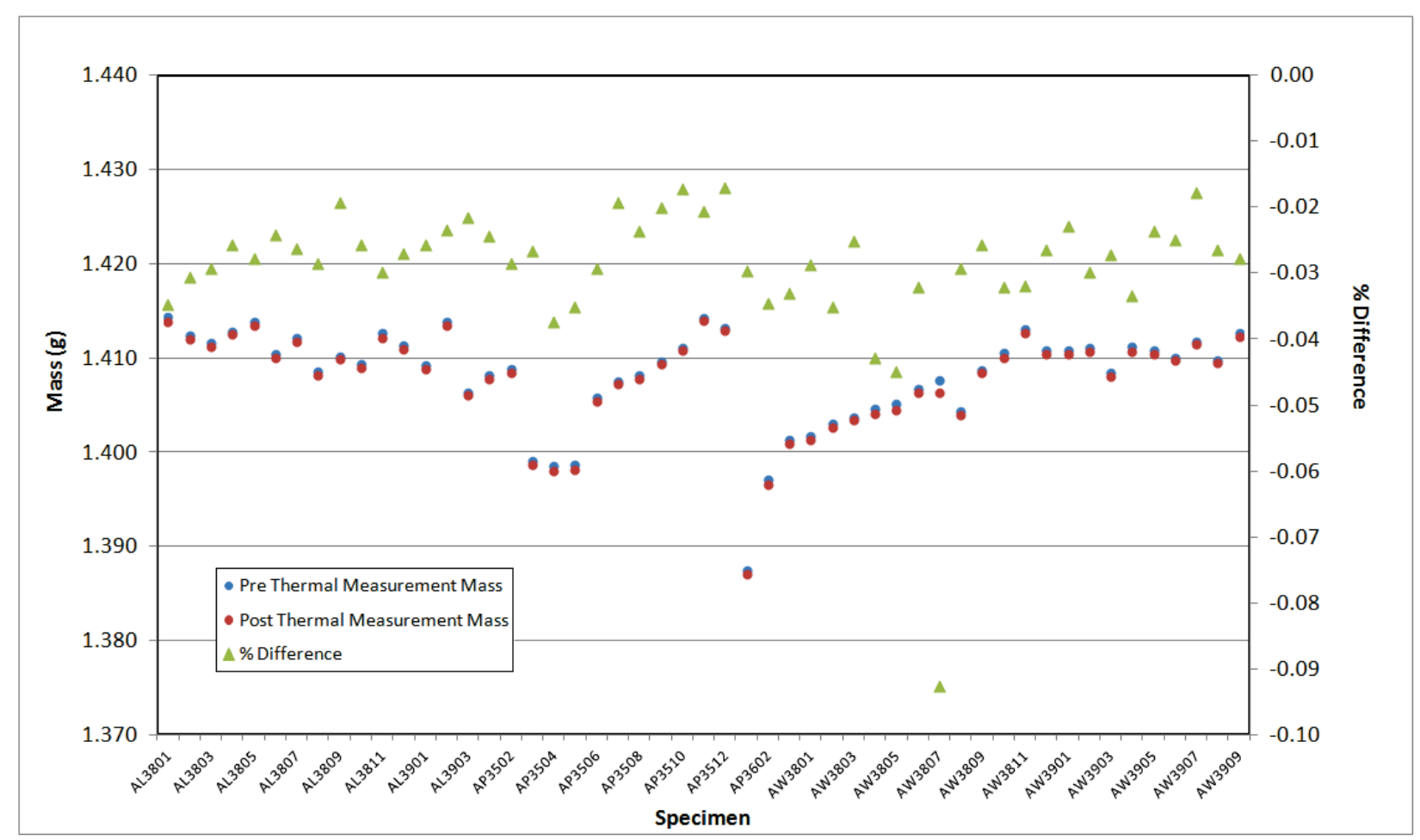

Figure A-108. NBG-17 Piggyback Pre vs. Post Thermal Measurement Mass Comparison.

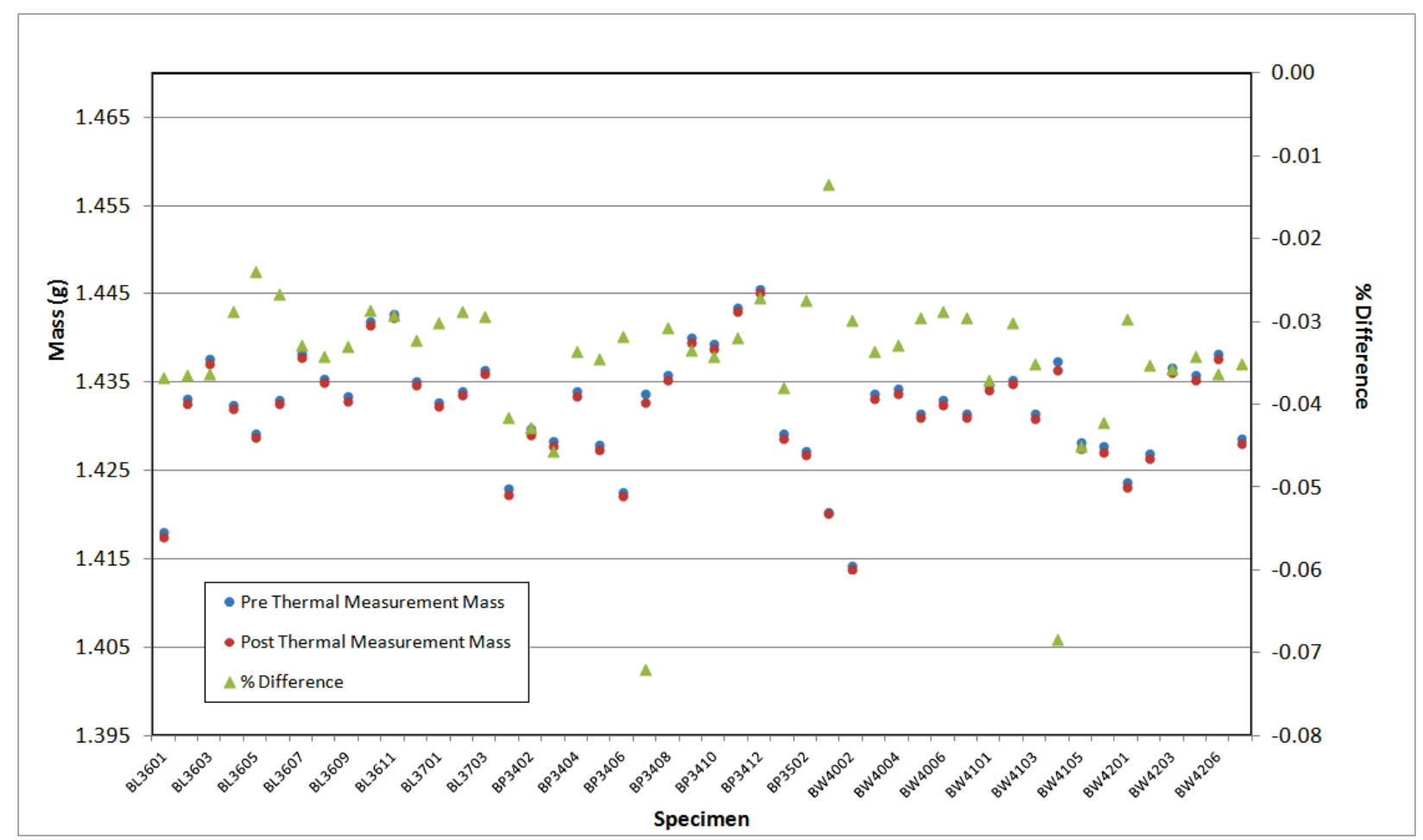

Figure A-109. NBG-18 Piggyback Pre vs. Post Thermal Measurement Mass Comparison. 


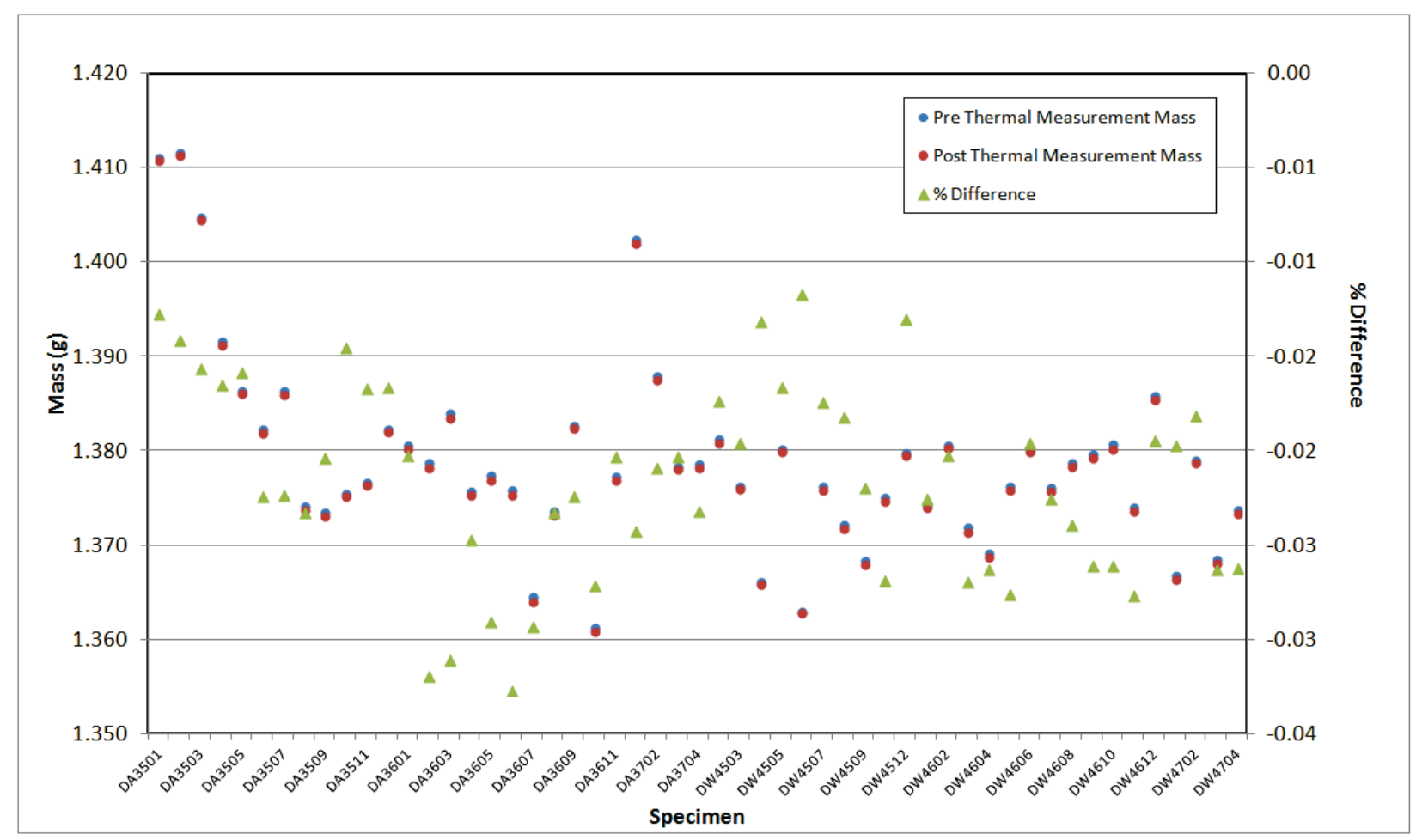

Figure A-110. PCEA Piggyback Pre vs. Post Thermal Measurement Mass Comparison.

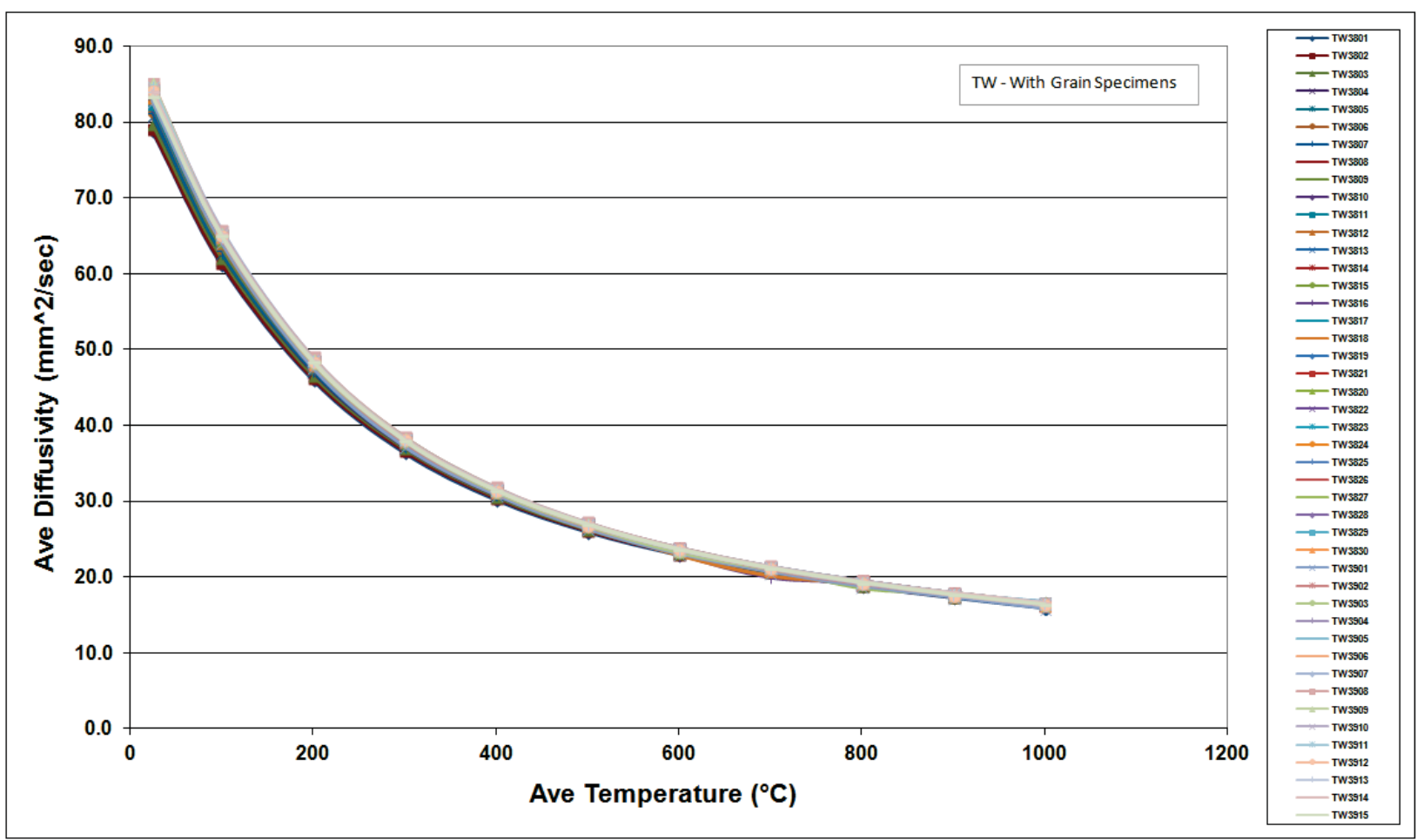

Figure A-111. 2114 Piggyback Diffusivity. 


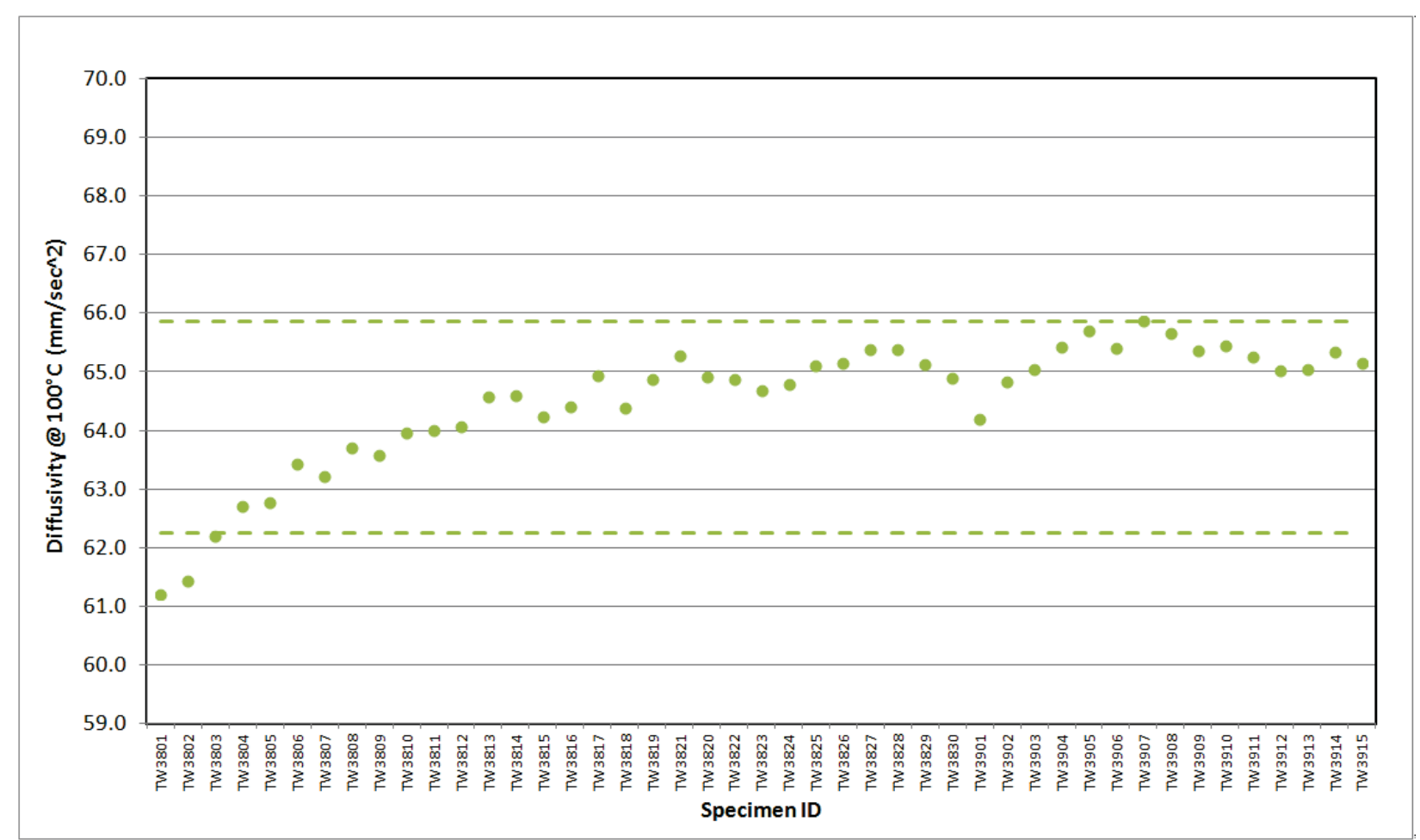

Figure A-112.2114 Piggyback Diffusivity @ 100 C.

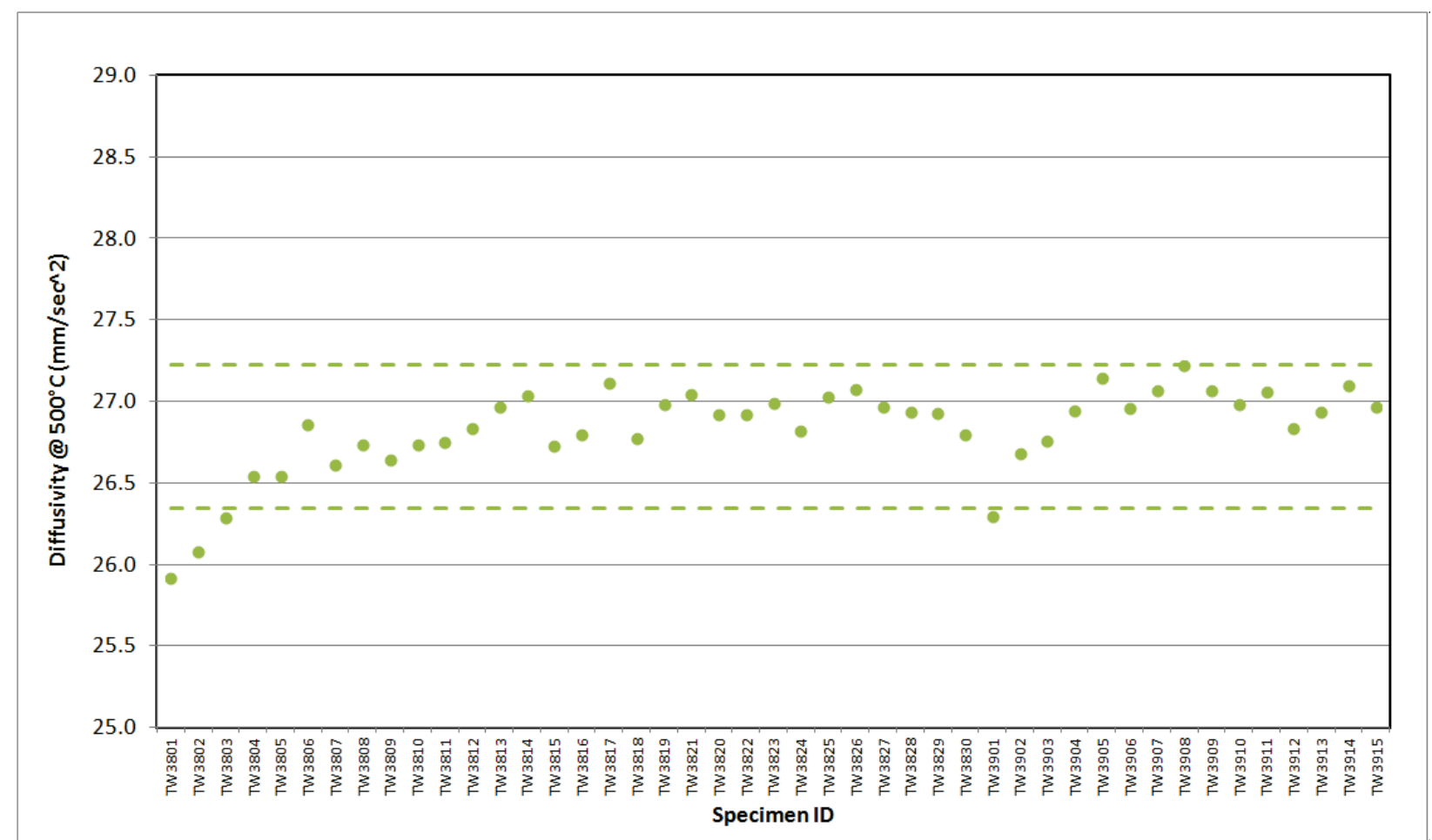

Figure A-113.2114 Piggyback Diffusivity @ 500 C. 


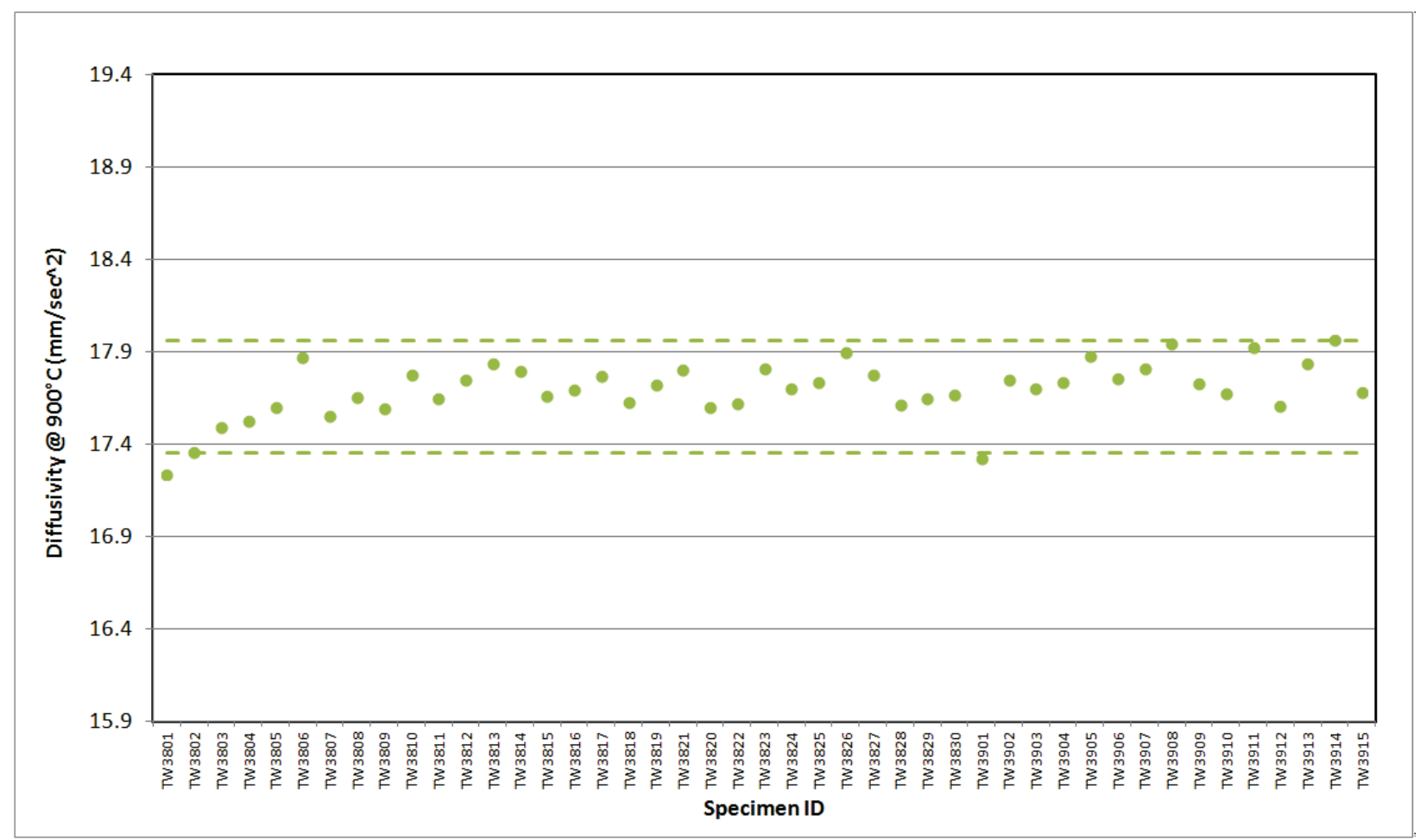

Figure A-114.2114 Piggyback Diffusivity @ $900^{\circ} \mathrm{C}$.

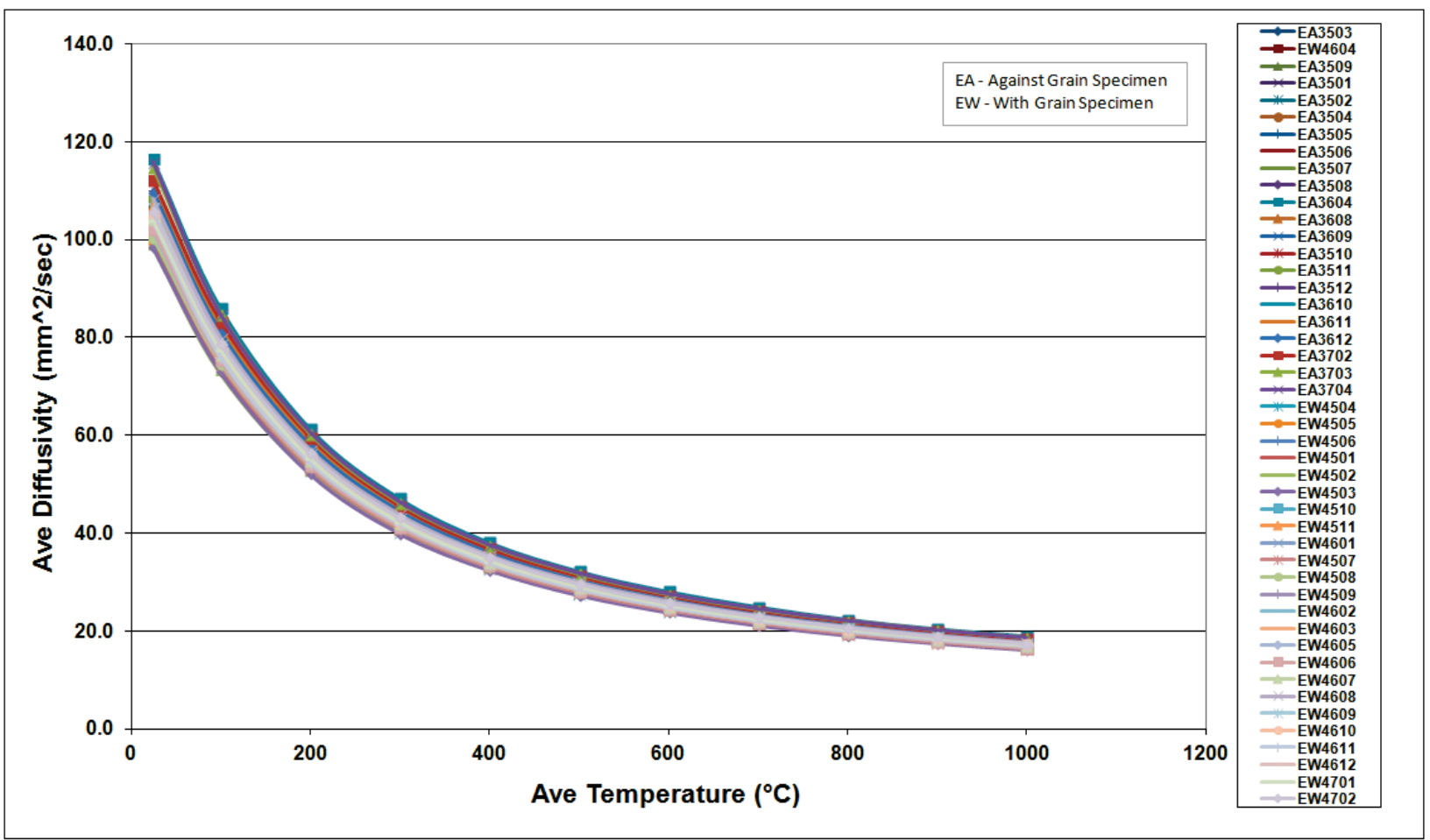

Figure A-115. IG-110 Piggyback Diffusivity. 


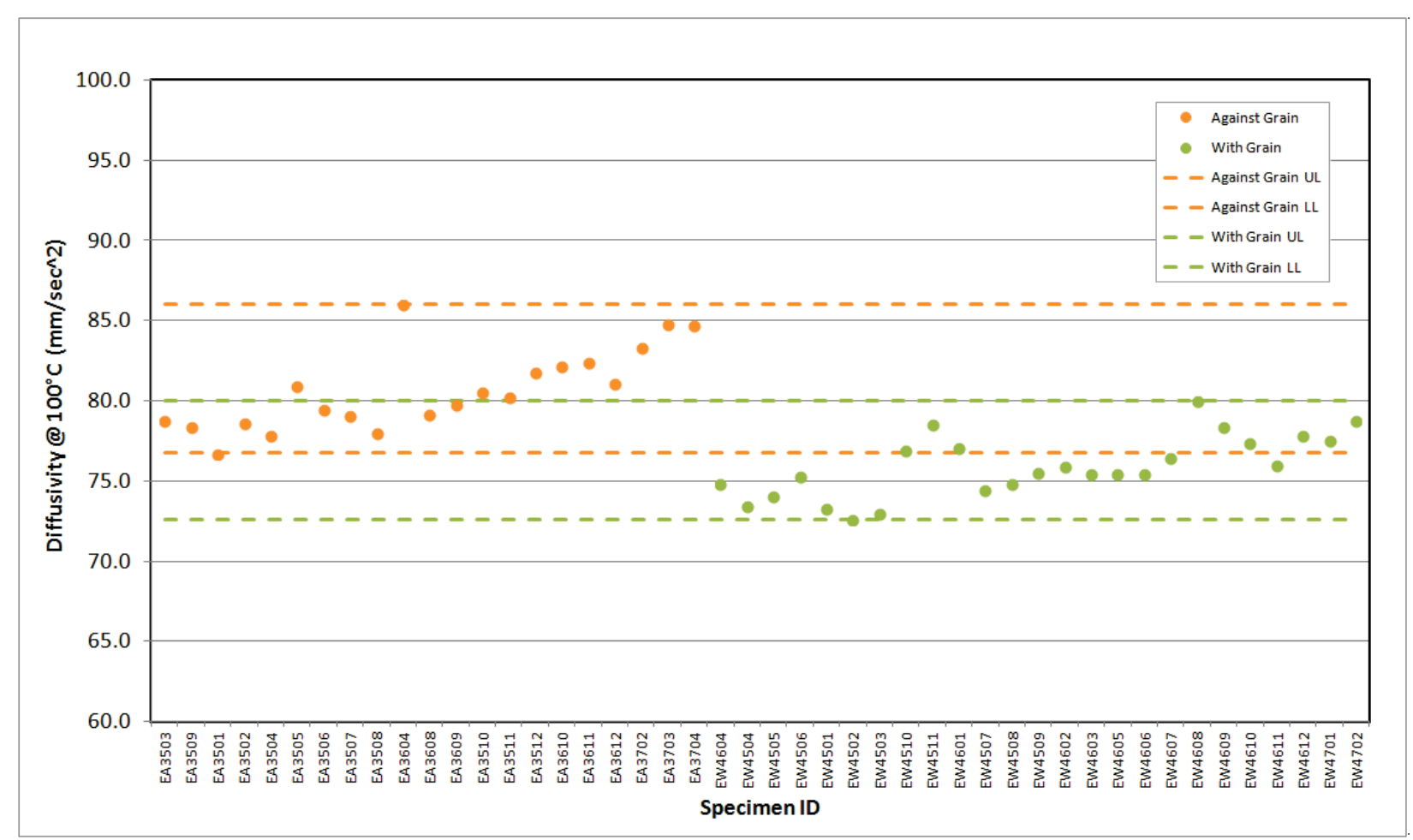

Figure A-116. IG-110 Piggyback Diffusivity @ $100^{\circ} \mathrm{C}$.

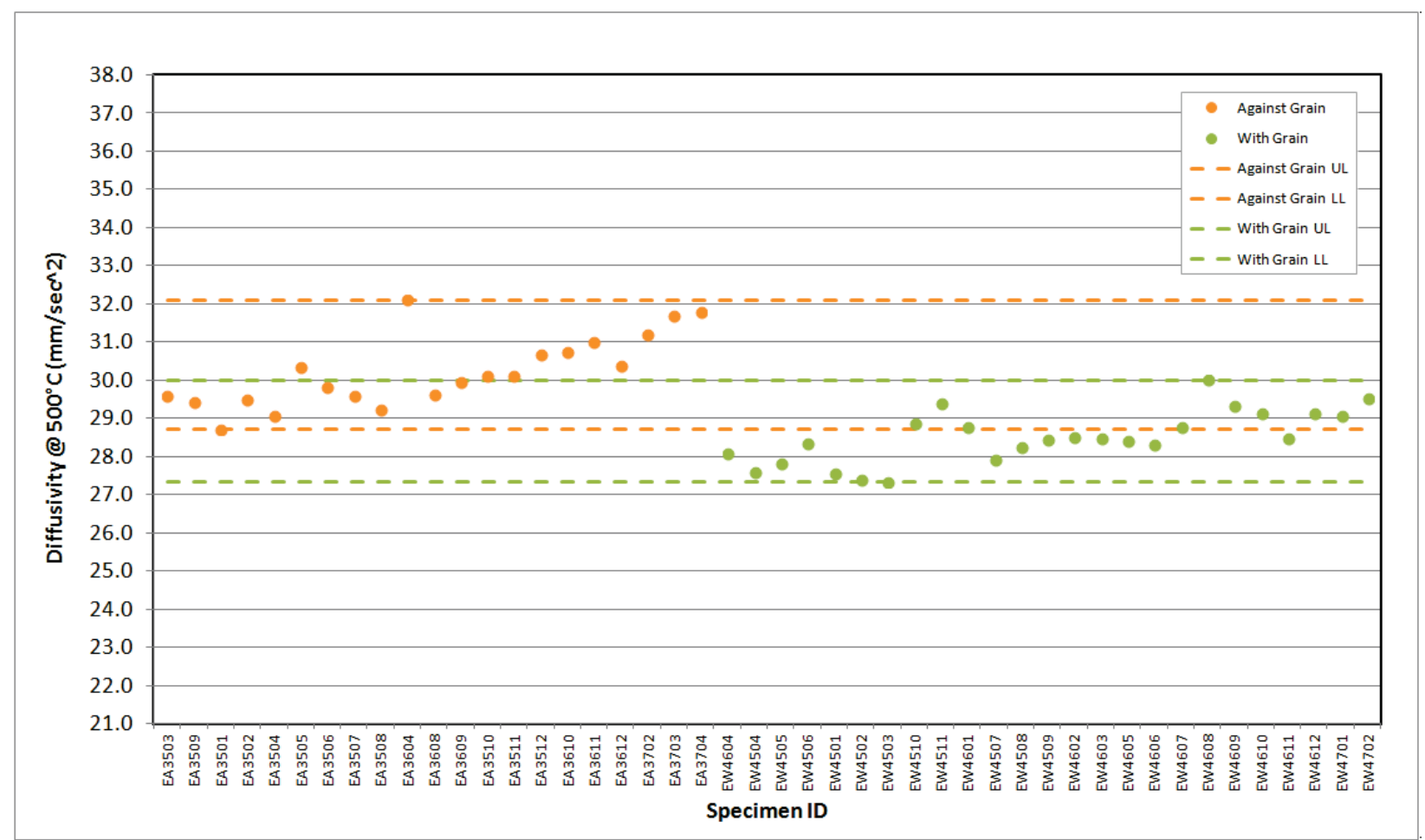

Figure A-117. IG-110 Piggyback Diffusivity @ 500․ 


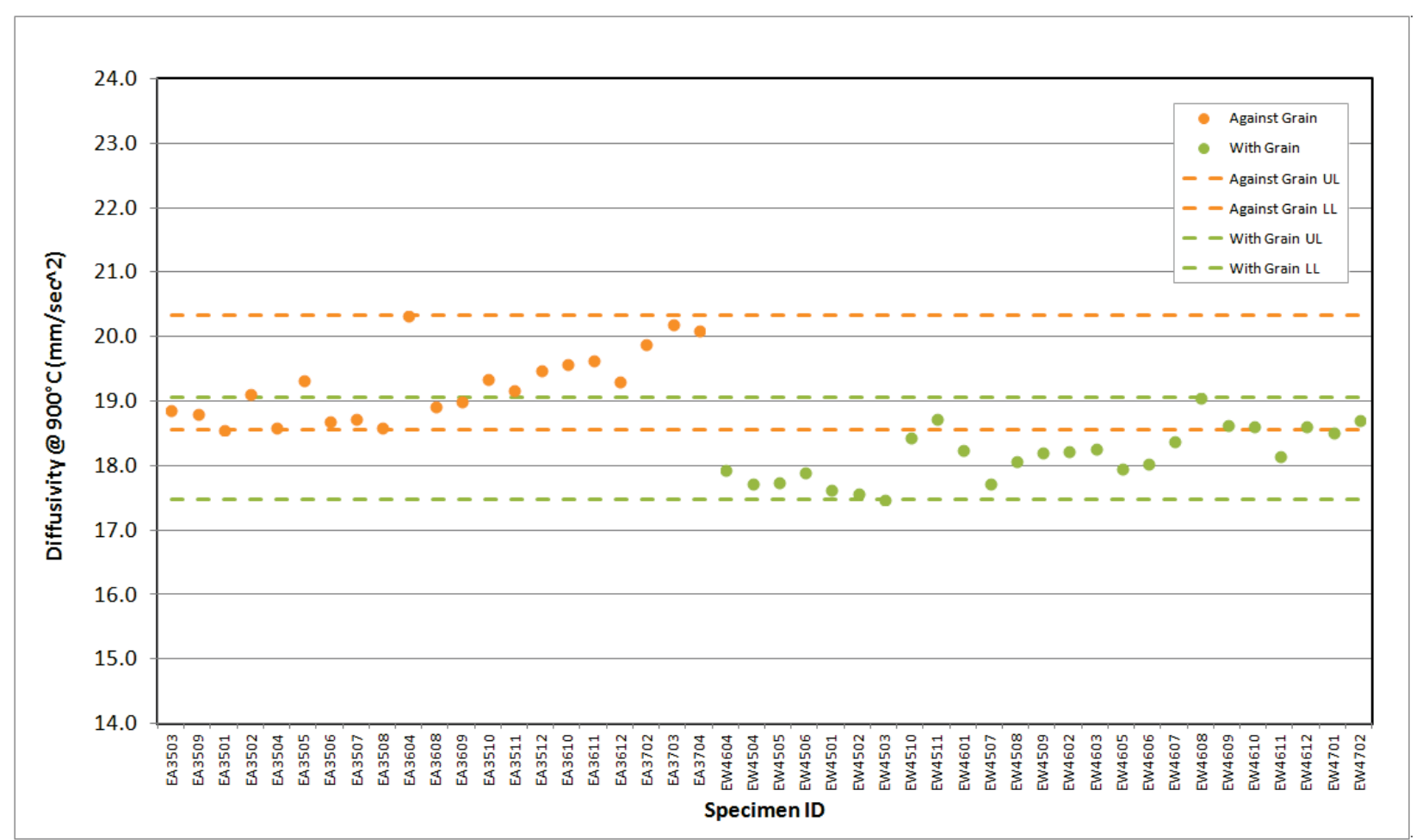

Figure A-118. IG-110 Piggyback Diffusivity @ 900².

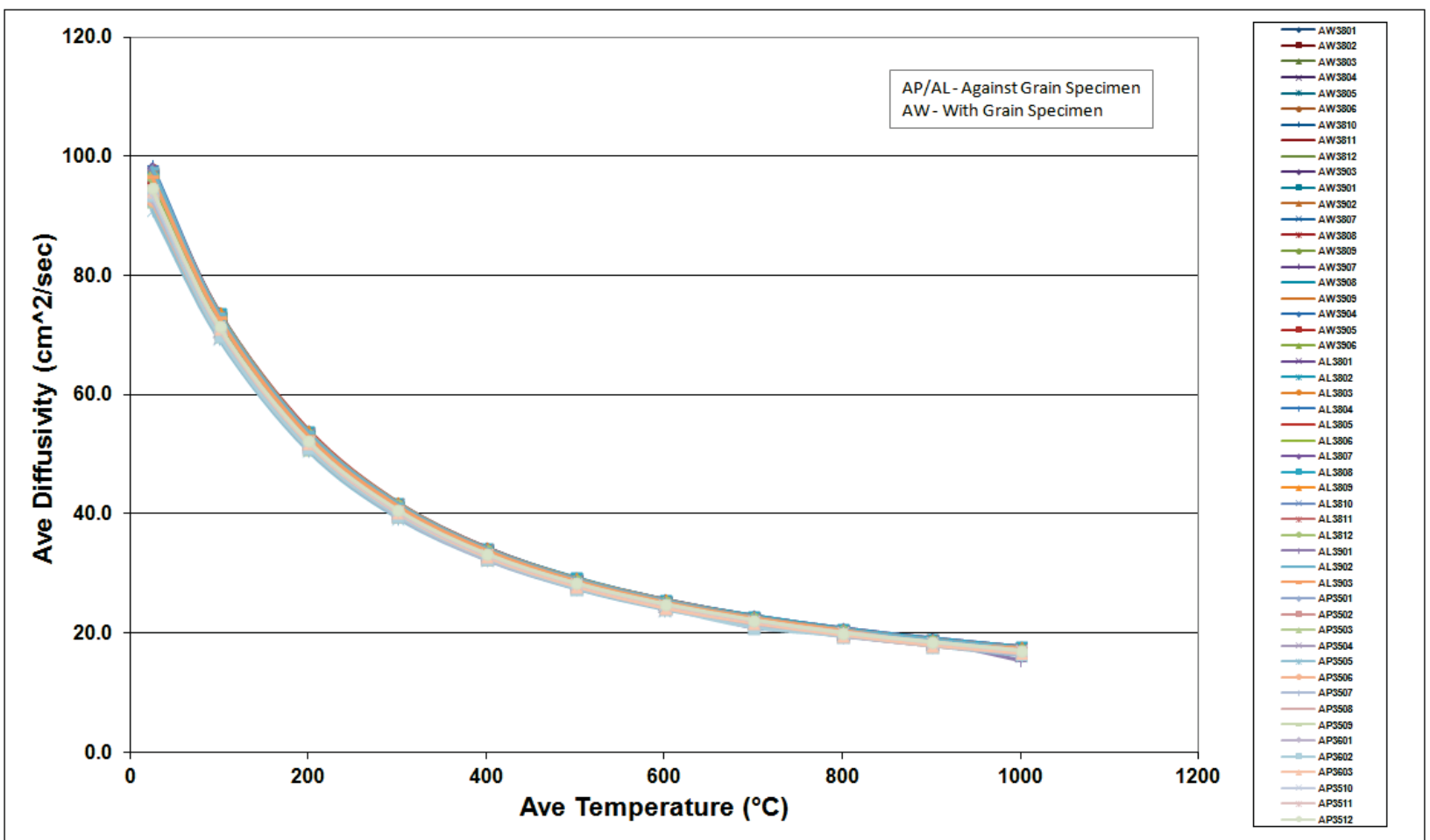

Figure A-119. NBG-17 Piggyback Diffusivity. 


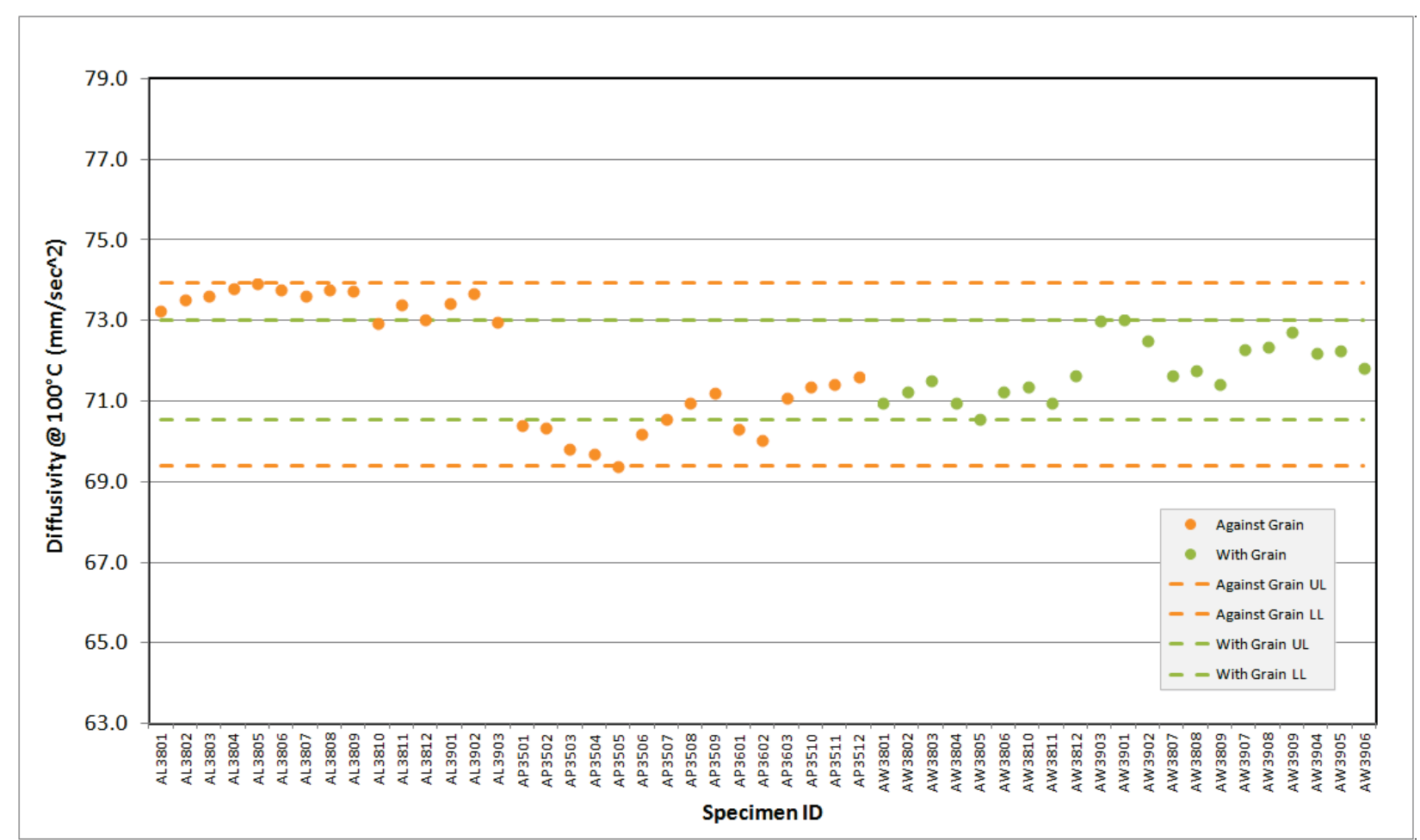

Figure A-120. NBG-17 Piggyback Diffusivity @ $100^{\circ} \mathrm{C}$.

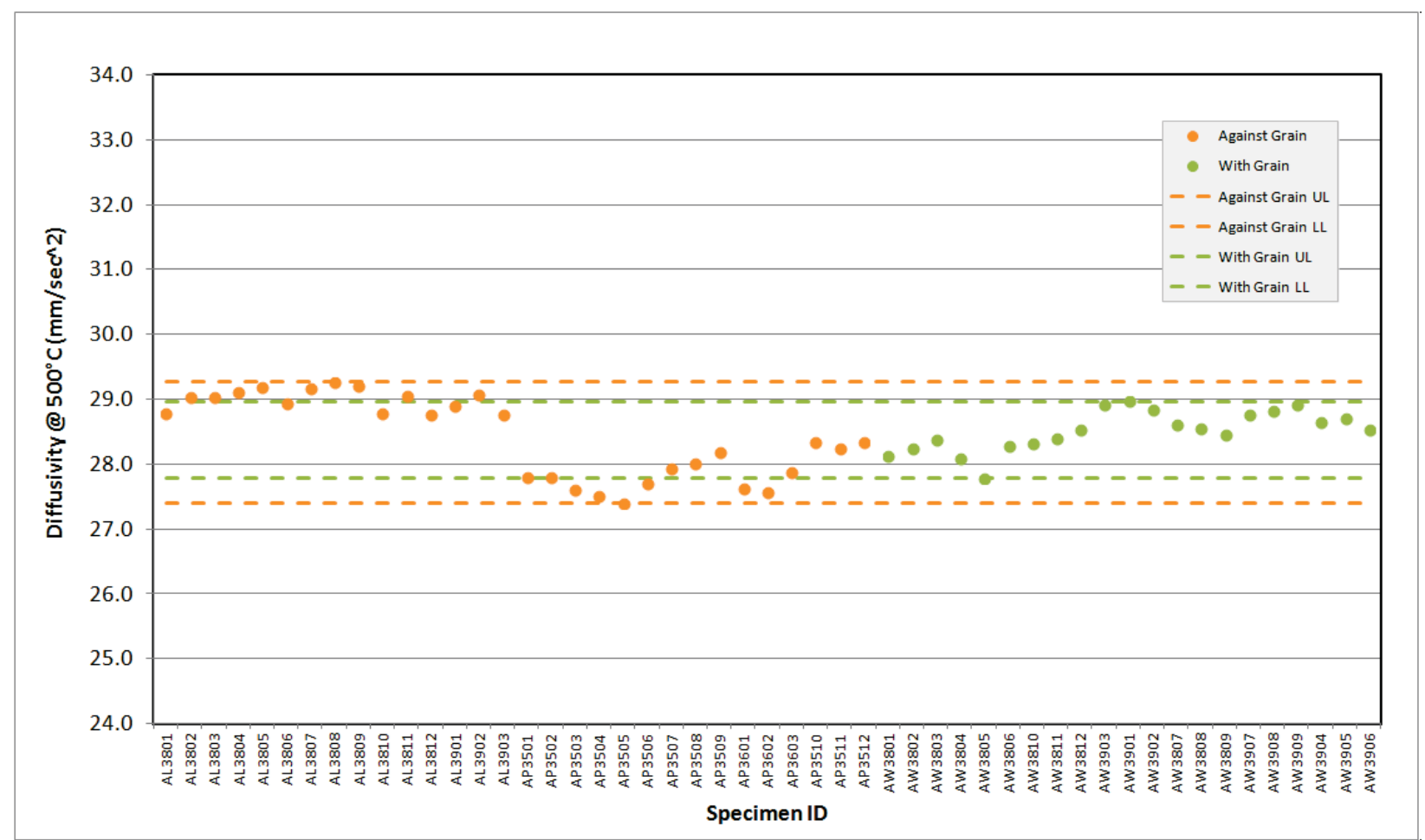

Figure A-121. NBG-17 Piggyback Diffusivity @ 500 C. 


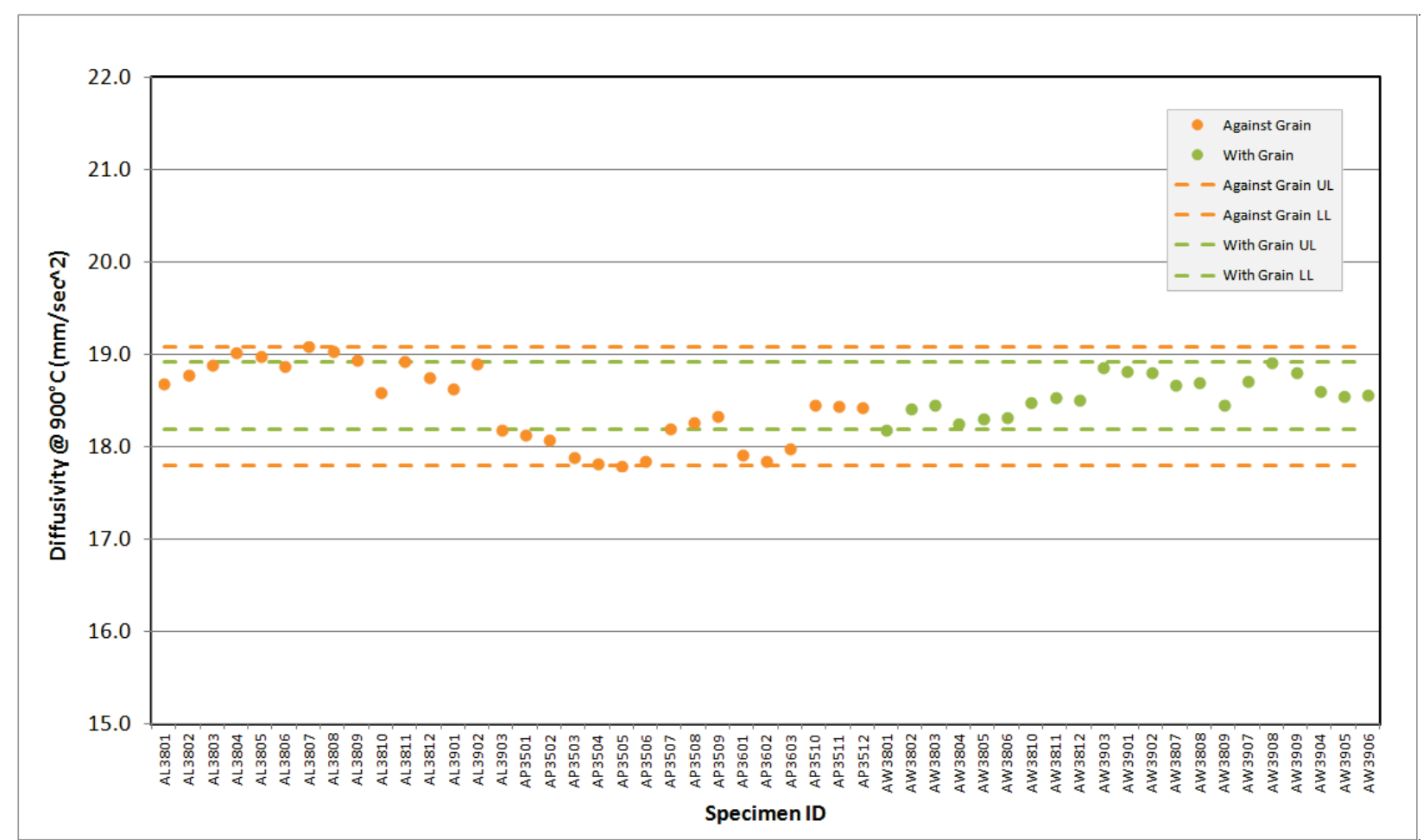

Figure A-122. NBG-17 Piggyback Diffusivity @ $900^{\circ} \mathrm{C}$.

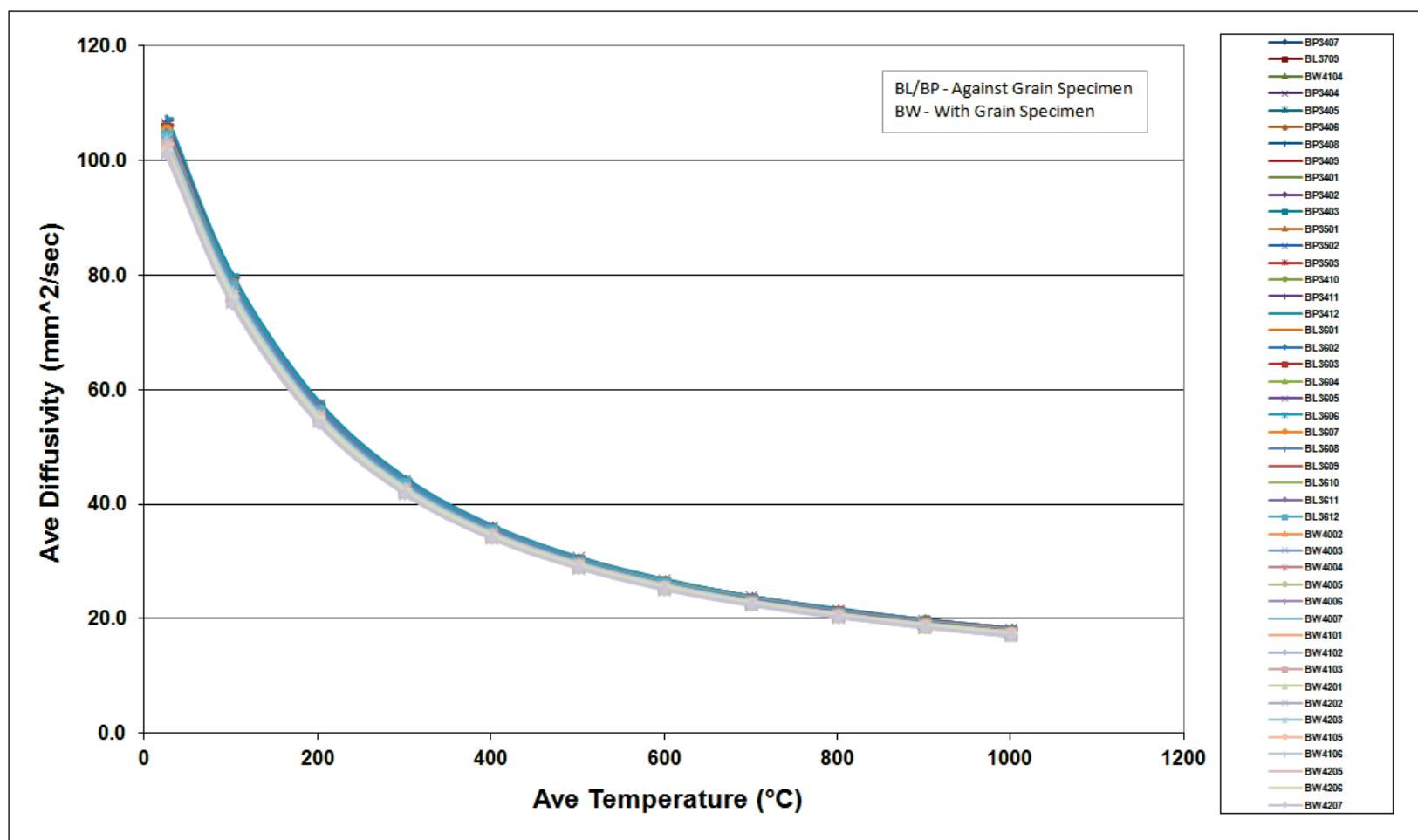

Figure A-123. NBG-18 Piggyback Diffusivity. 


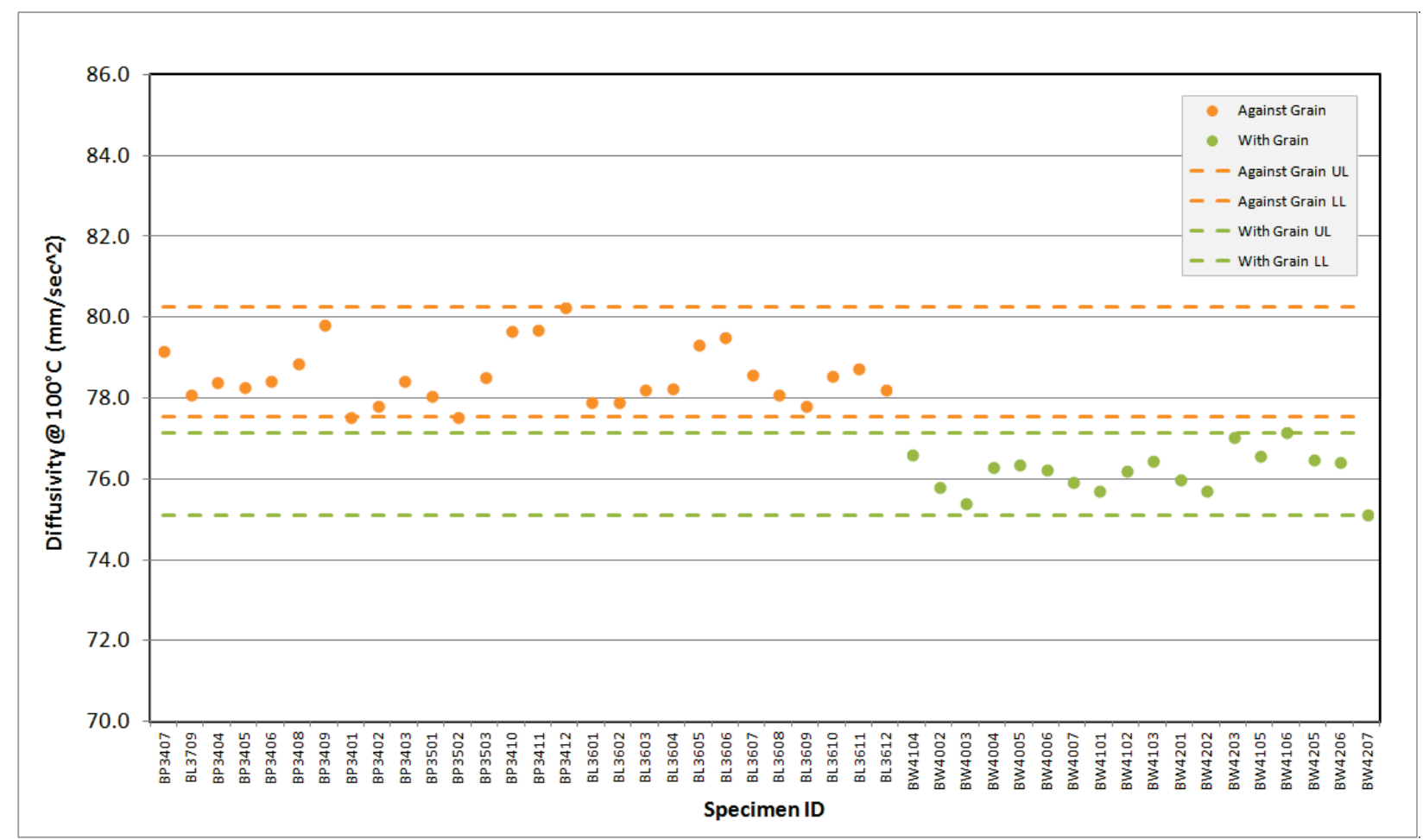

Figure A-124. NBG-18 Piggyback Diffusivity @ $100^{\circ} \mathrm{C}$.

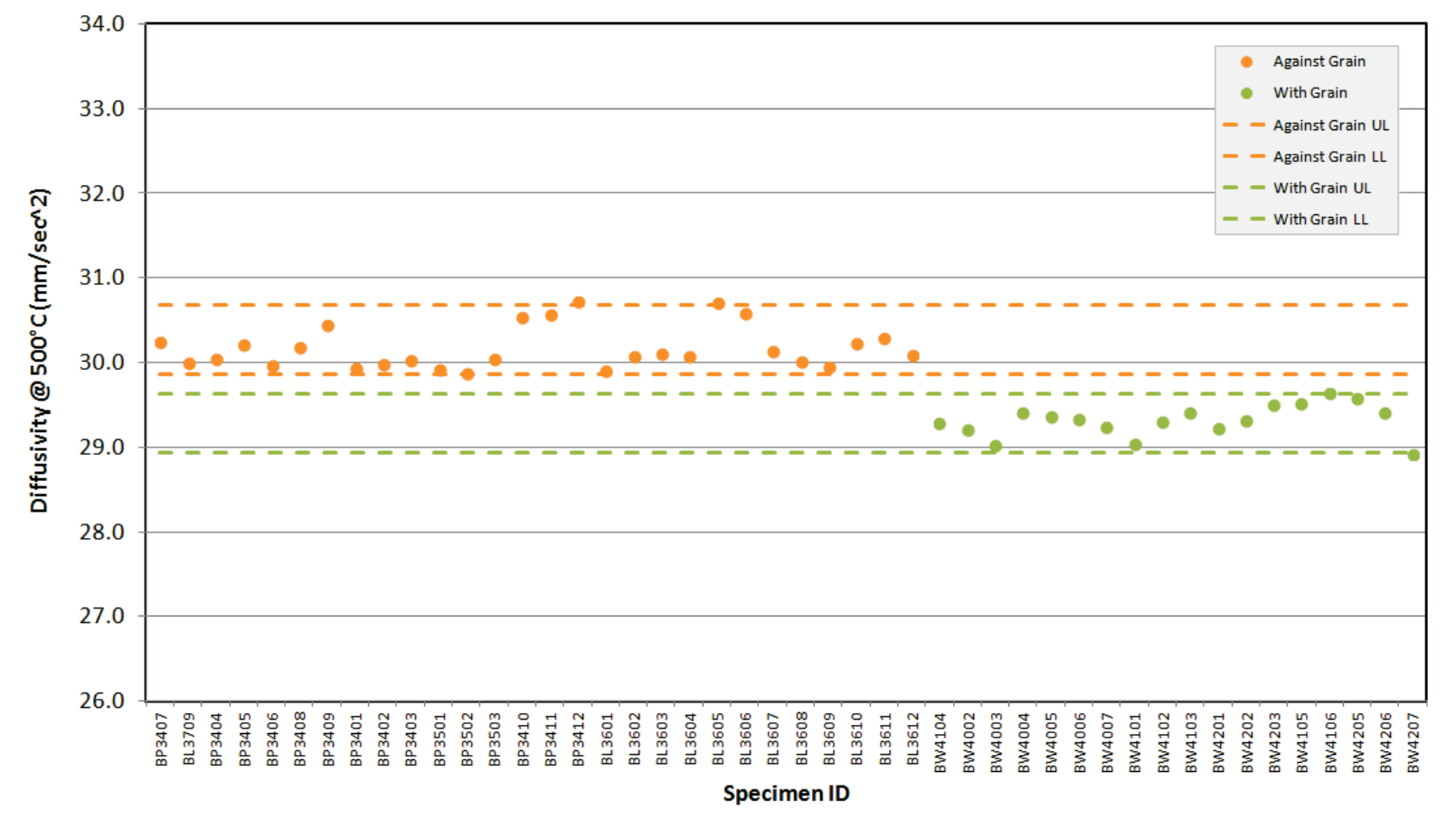

Figure A-125. NBG-18 Piggyback Diffusivity @ $500^{\circ} \mathrm{C}$. 


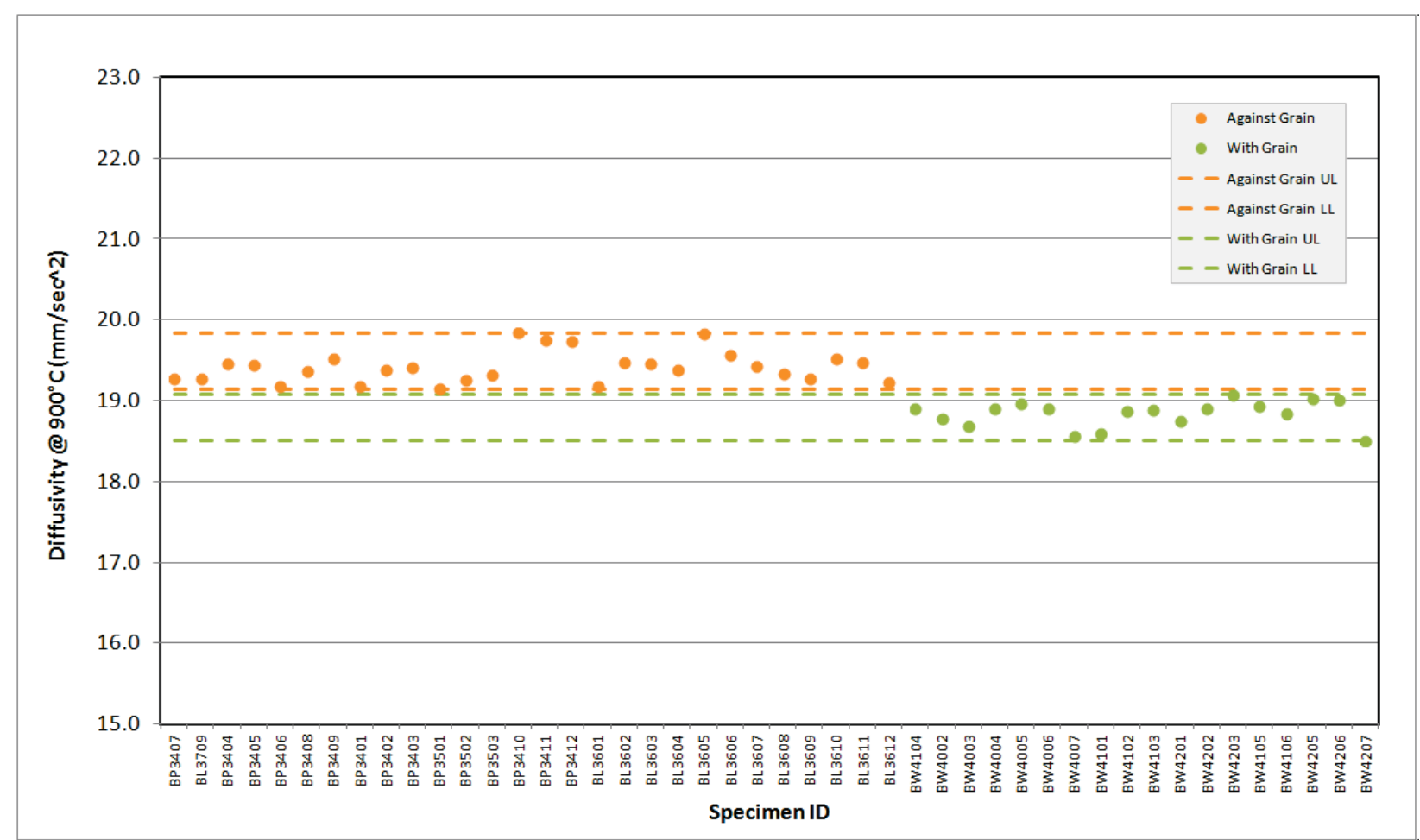

Figure A-126. NBG-18 Diffusivity@900 C.

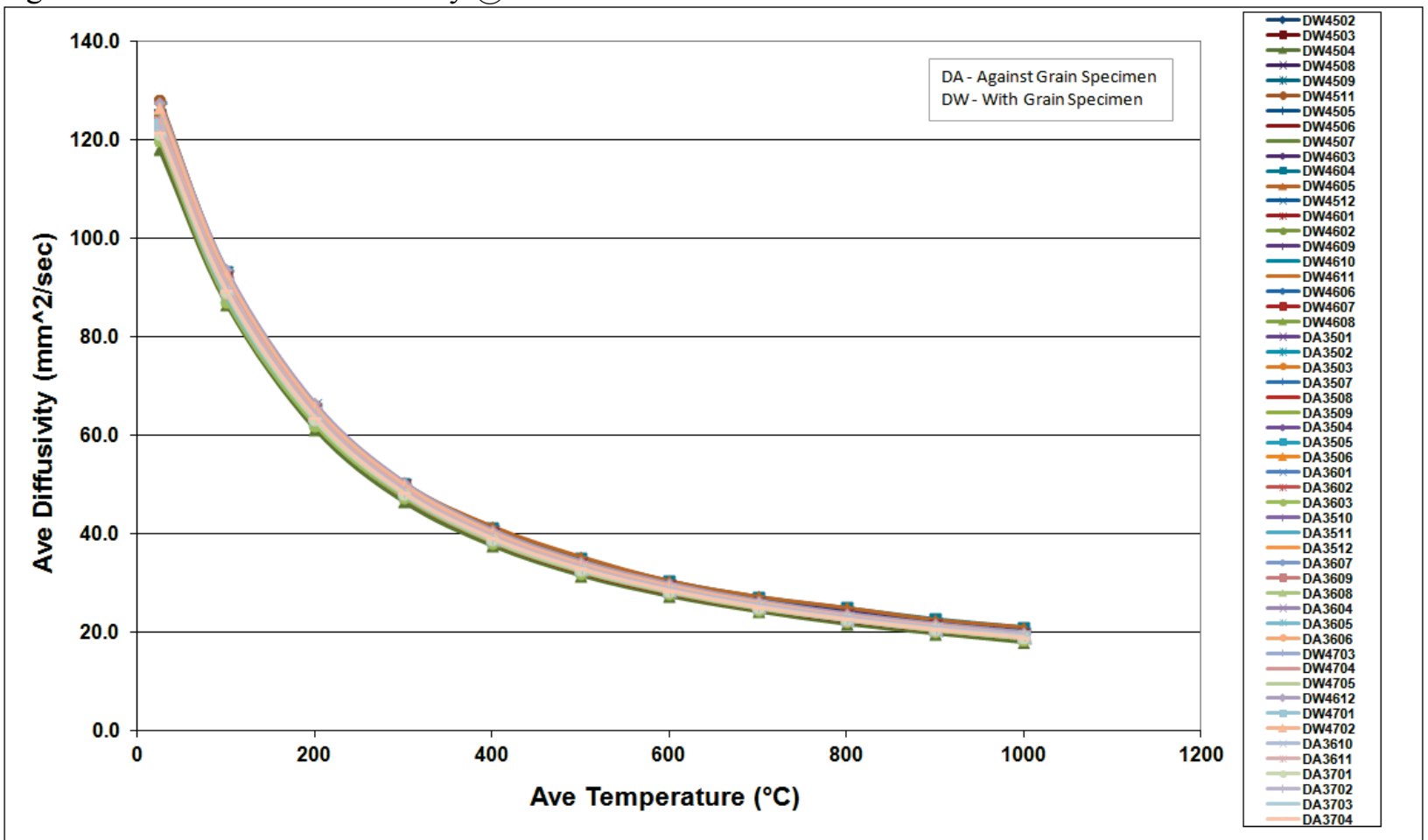

Figure A-127. PCEA Piggyback Diffusivity. 


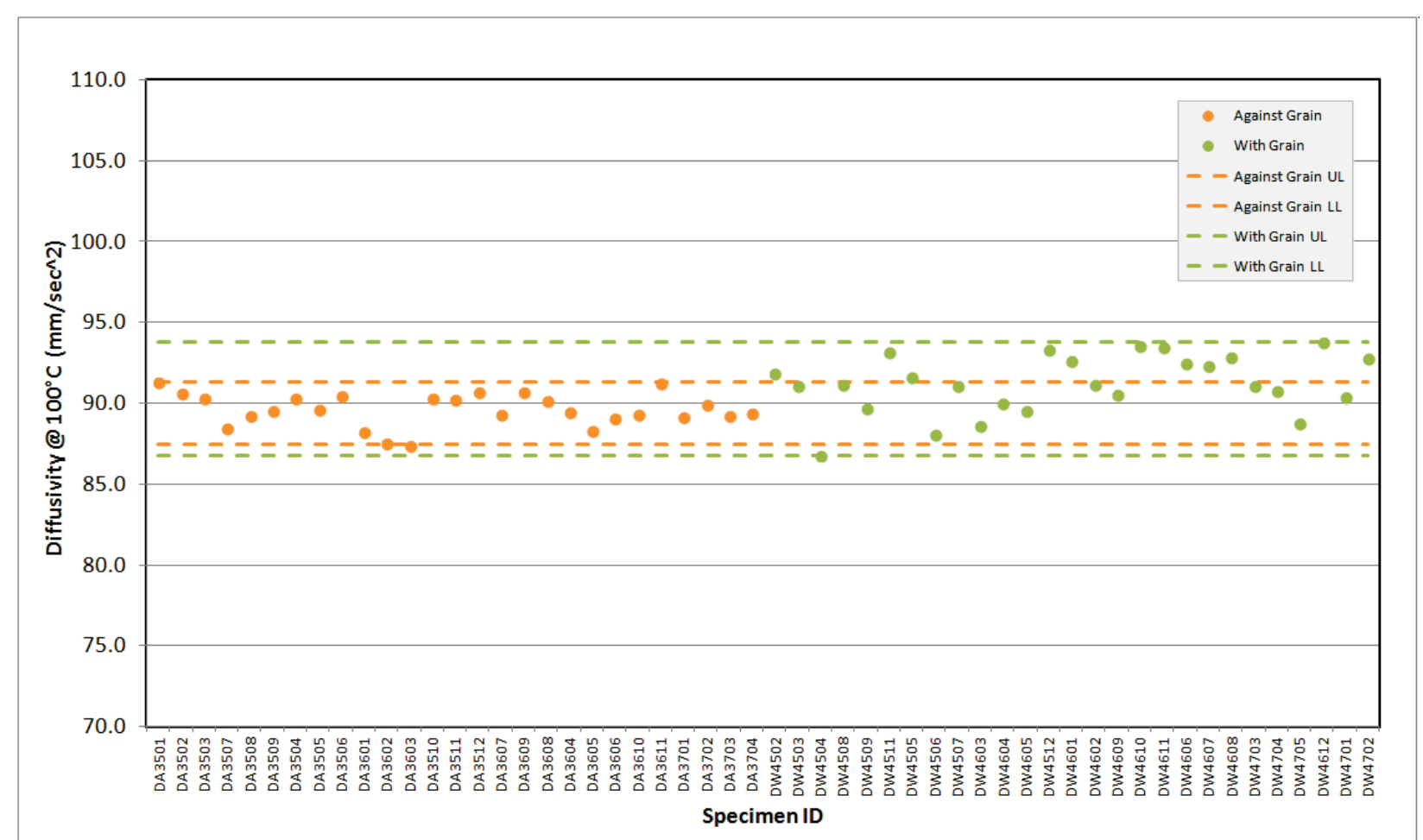

Figure A-128. PCEA Piggyback Diffusivity @ $100^{\circ} \mathrm{C}$.

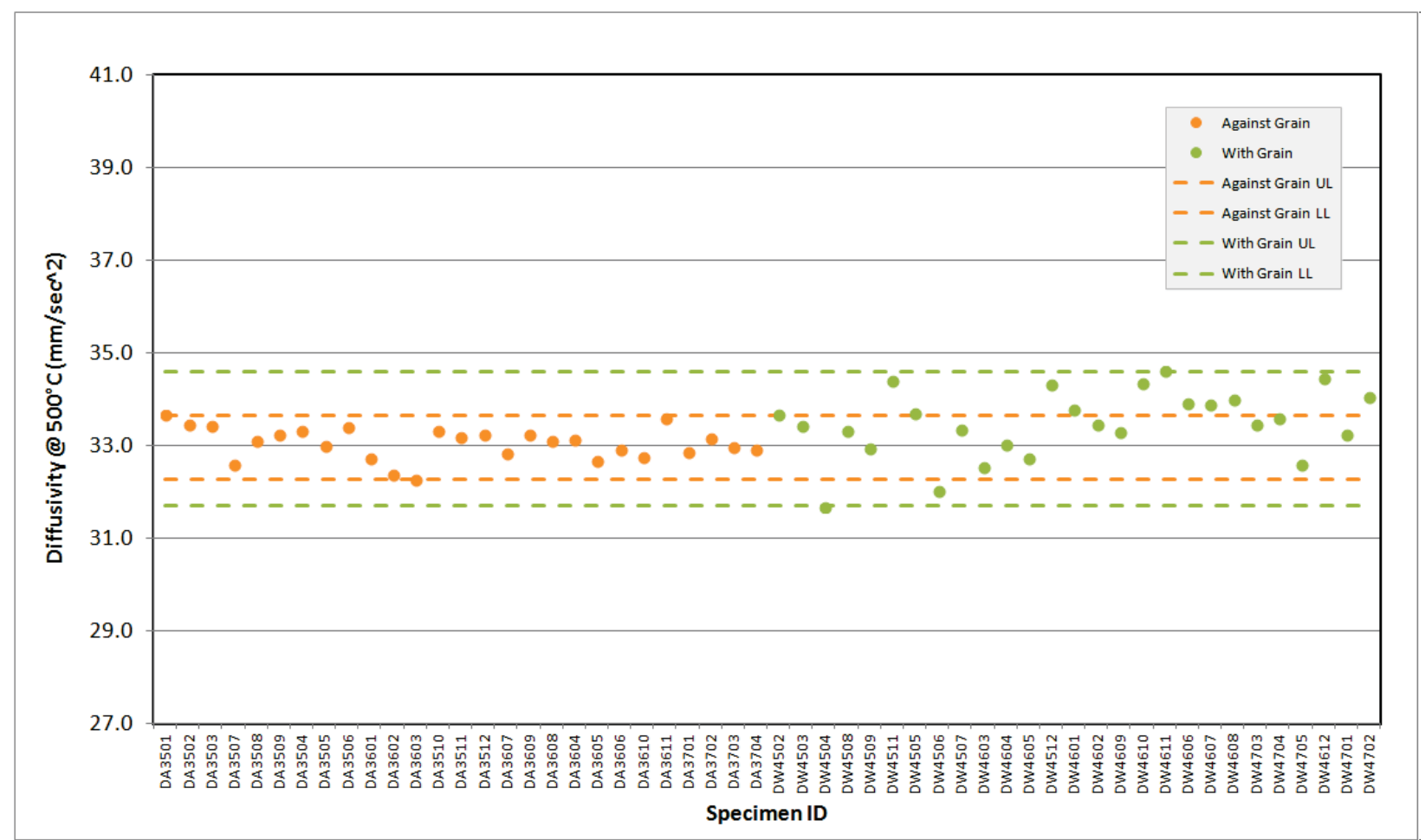

Figure A-129. PCEA Piggyback Diffusivity @ $500^{\circ} \mathrm{C}$. 


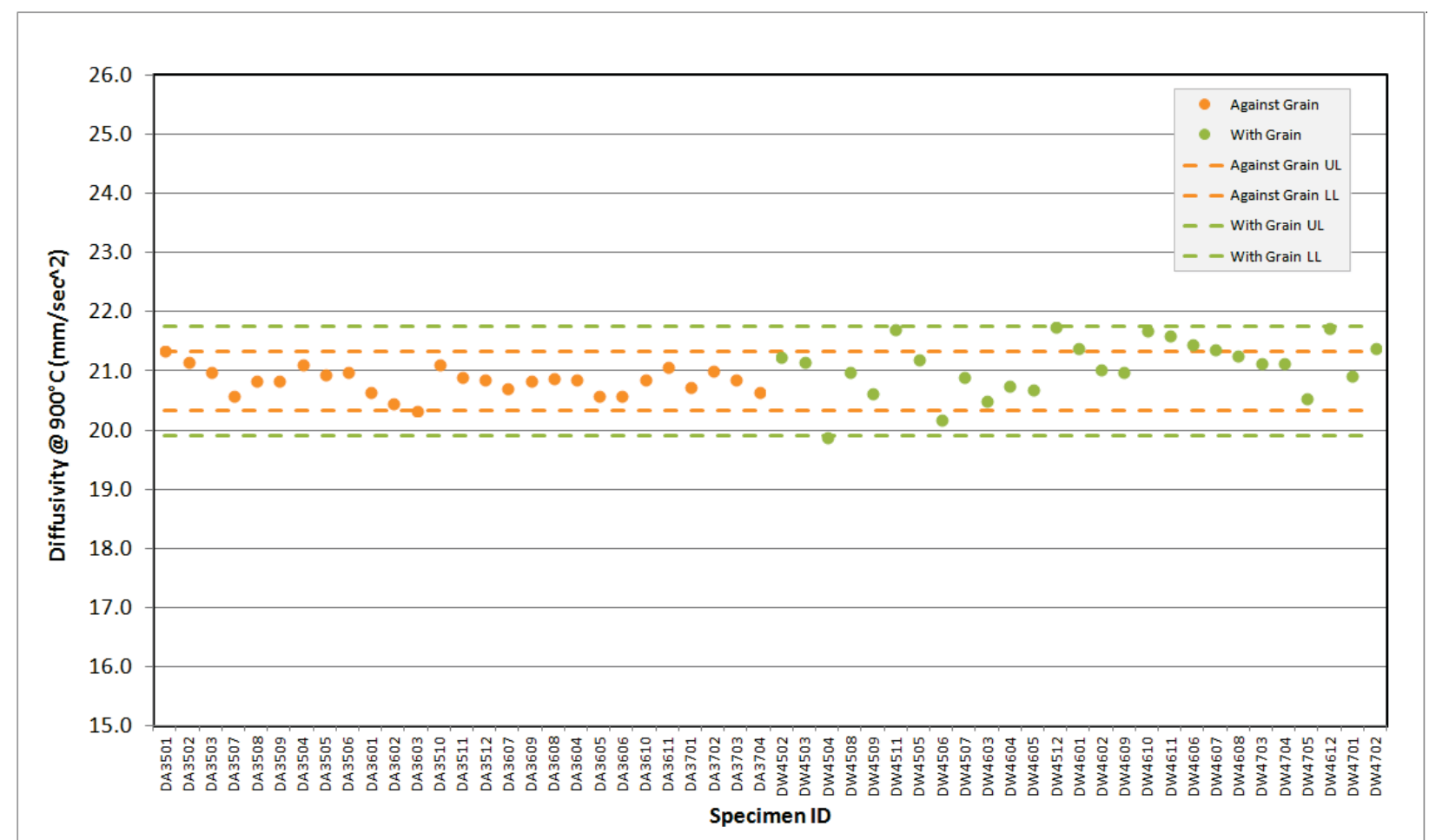

Figure A-130. PCEA Piggyback Diffusivity @ $900^{\circ} \mathrm{C}$. 


\section{Appendix B Summary of Statistical Parameters}

Table B-1. Creep Pre Thermal Measurement Length ( $\mathrm{mm})$ Summary Statistics.

\begin{tabular}{|c|c|c|c|c|c|c|}
\hline $\begin{array}{l}\text { Combined } \\
\text { Specimens }\end{array}$ & Mean & Std Dev & $\operatorname{CoV}(\%)$ & Median & $\begin{array}{l}\text { Upper } \\
\text { Limit }\end{array}$ & $\begin{array}{c}\text { Lower } \\
\text { Limit }\end{array}$ \\
\hline IG-110 & 25.3745 & 0.0093 & 0.04 & 25.3753 & 25.3872 & 25.3641 \\
\hline NBG-17 & 25.3601 & 0.0106 & 0.04 & 25.3591 & 25.3823 & 25.3344 \\
\hline NBG-18 & 25.3719 & 0.1179 & 0.46 & 25.3930 & 25.4800 & 25.2730 \\
\hline PCEA & 25.3657 & 0.0414 & 0.16 & 25.3763 & 25.3990 & 25.3359 \\
\hline 2114 & 25.3820 & 0.0075 & 0.03 & 25.3826 & 25.3975 & 25.3655 \\
\hline $\begin{array}{c}\text { Against } \\
\text { Grain } \\
\text { Specimens }\end{array}$ & Mean & Std Dev & $\mathrm{CoV}(\%)$ & Median & $\begin{array}{l}\text { Upper } \\
\text { Limit }\end{array}$ & $\begin{array}{r}\text { Lower } \\
\text { Limit }\end{array}$ \\
\hline IG-110 & 25.3679 & 0.0164 & 0.06 & 25.3703 & 25.3951 & 25.3405 \\
\hline NBG-17 & 25.3612 & 0.0117 & 0.05 & 25.3616 & 25.3823 & 25.3321 \\
\hline NBG-18 & 25.3704 & 0.1329 & 0.52 & 25.3924 & 25.4669 & 25.3091 \\
\hline PCEA & 25.3627 & 0.0238 & 0.09 & 25.3695 & 25.3910 & 25.3153 \\
\hline 2114 & & & & & & \\
\hline $\begin{array}{l}\text { With Grain } \\
\text { Specimens }\end{array}$ & Mean & Std Dev & $\mathrm{CoV}(\%)$ & Median & $\begin{array}{l}\text { Upper } \\
\text { Limit }\end{array}$ & $\begin{array}{c}\text { Lower } \\
\text { Limit }\end{array}$ \\
\hline IG-110 & 25.3766 & 0.0033 & 0.01 & 25.3765 & 25.3840 & 25.3698 \\
\hline NBG-17 & 25.3584 & 0.0082 & 0.03 & 25.3576 & 25.3738 & 25.3413 \\
\hline NBG-18 & 25.3752 & 0.0781 & 0.31 & 25.3965 & 25.4800 & 25.1889 \\
\hline PCEA & 25.3667 & 0.0460 & 0.18 & 25.3780 & 25.3990 & 25.3511 \\
\hline 2114 & 25.3820 & 0.0075 & 0.03 & 25.3826 & 25.3975 & 25.3655 \\
\hline
\end{tabular}


Table B-2. Creep Pre Thermal Measurement Diameter (mm) Summary Statistics.

\begin{tabular}{|c|c|c|c|c|c|c|}
\hline $\begin{array}{l}\text { Combined } \\
\text { Specimens }\end{array}$ & Mean & Std Dev & $\mathrm{CoV}(\%)$ & Median & $\begin{array}{l}\text { Upper } \\
\text { Limit }\end{array}$ & $\begin{array}{c}\text { Lower } \\
\text { Limit }\end{array}$ \\
\hline IG-110 & 12.5034 & 0.0192 & 0.15 & 12.5089 & 12.5311 & 12.4484 \\
\hline NBG-17 & 12.4630 & 0.0059 & 0.05 & 12.4639 & 12.4718 & 12.4492 \\
\hline NBG-18 & 12.4878 & 0.0102 & 0.08 & 12.4883 & 12.5103 & 12.4606 \\
\hline PCEA & 12.4722 & 0.0058 & 0.05 & 12.4732 & 12.4821 & 12.4581 \\
\hline 2114 & 12.4759 & 0.0072 & 0.06 & 12.4765 & 12.4885 & 12.4599 \\
\hline $\begin{array}{c}\text { Against } \\
\text { Grain } \\
\text { Specimens }\end{array}$ & Mean & Std Dev & $\mathrm{CoV}(\%)$ & Median & $\begin{array}{l}\text { Upper } \\
\text { Limit }\end{array}$ & $\begin{array}{c}\text { Lower } \\
\text { Limit }\end{array}$ \\
\hline IG-110 & 12.4804 & 0.0154 & 0.12 & 12.4852 & 12.4998 & 12.4438 \\
\hline NBG-17 & 12.4616 & 0.0064 & 0.05 & 12.4627 & 12.4710 & 12.4460 \\
\hline NBG-18 & 12.4910 & 0.0100 & 0.08 & 12.4917 & 12.5103 & 12.4653 \\
\hline PCEA & 12.4682 & 0.0066 & 0.05 & 12.4680 & 12.4791 & 12.4543 \\
\hline 2114 & & & & & & \\
\hline $\begin{array}{l}\text { With Grain } \\
\text { Specimens }\end{array}$ & Mean & Std Dev & $\mathrm{CoV}(\%)$ & Median & $\begin{array}{l}\text { Upper } \\
\text { Limit }\end{array}$ & $\begin{array}{l}\text { Lower } \\
\text { Limit }\end{array}$ \\
\hline IG-110 & 12.5111 & 0.0133 & 0.11 & 12.5131 & 12.5311 & 12.4798 \\
\hline NBG-17 & 12.4652 & 0.0043 & 0.03 & 12.4657 & 12.4718 & 12.4541 \\
\hline NBG-18 & 12.4808 & 0.0066 & 0.05 & 12.4795 & 12.4950 & 12.4664 \\
\hline PCEA & 12.4736 & 0.0049 & 0.04 & 12.4740 & 12.4821 & 12.4623 \\
\hline 2114 & 12.4759 & 0.0072 & 0.06 & 12.4765 & 12.4885 & 12.4599 \\
\hline
\end{tabular}


Table B-3.Creep Pre Thermal Measurement Mass (g) Summary Statistics.

\begin{tabular}{|c|c|c|c|c|c|c|}
\hline $\begin{array}{l}\text { Combined } \\
\text { Specimens }\end{array}$ & Mean & Std Dev & $\operatorname{CoV}(\%)$ & Median & $\begin{array}{l}\text { Upper } \\
\text { Limit }\end{array}$ & $\begin{array}{l}\text { Lower } \\
\text { Limit }\end{array}$ \\
\hline IG-110 & 5.4004 & 0.0326 & 0.60 & 5.4071 & 5.4565 & 5.3214 \\
\hline NBG-17 & 5.5592 & 0.0229 & 0.41 & 5.5541 & 5.6039 & 5.5180 \\
\hline NBG-18 & 5.6561 & 0.0302 & 0.53 & 5.6585 & 5.7088 & 5.6027 \\
\hline PCEA & 5.4215 & 0.0262 & 0.48 & 5.4198 & 5.4577 & 5.3819 \\
\hline 2114 & 5.5339 & 0.0125 & 0.23 & 5.5355 & 5.5551 & 5.5061 \\
\hline $\begin{array}{c}\text { Against } \\
\text { Grain } \\
\text { Specimens }\end{array}$ & Mean & Std Dev & $\mathrm{CoV}(\%)$ & Median & $\begin{array}{l}\text { Upper } \\
\text { Limit }\end{array}$ & $\begin{array}{r}\text { Lower } \\
\text { Limit }\end{array}$ \\
\hline IG-110 & 5.3582 & 0.0295 & 0.55 & 5.3644 & 5.4011 & 5.2961 \\
\hline NBG-17 & 5.5655 & 0.0261 & 0.47 & 5.5655 & 5.6039 & 5.5180 \\
\hline NBG-18 & 5.6647 & 0.0310 & 0.55 & 5.6662 & 5.7088 & 5.6178 \\
\hline PCEA & 5.4468 & 0.0336 & 0.62 & 5.4377 & 5.4937 & 5.4132 \\
\hline 2114 & & & & & & \\
\hline $\begin{array}{l}\text { With Grain } \\
\text { Specimens }\end{array}$ & Mean & Std Dev & $\operatorname{CoV}(\%)$ & Median & $\begin{array}{c}\text { Upper } \\
\text { Limit }\end{array}$ & $\begin{array}{r}\text { Lower } \\
\text { Limit } \\
\end{array}$ \\
\hline IG-110 & 5.4145 & 0.0185 & 0.34 & 5.4145 & 5.4565 & 5.3755 \\
\hline NBG-17 & 5.5490 & 0.0104 & 0.19 & 5.5458 & 5.5715 & 5.5341 \\
\hline NBG-18 & 5.6373 & 0.0171 & 0.30 & 5.6384 & 5.6654 & 5.6032 \\
\hline PCEA & 5.4130 & 0.0164 & 0.30 & 5.4162 & 5.4395 & 5.3788 \\
\hline 2114 & 5.5339 & 0.0125 & 0.23 & 5.5355 & 5.5551 & 5.5061 \\
\hline
\end{tabular}


Table B-4. Creep Pre Thermal Measurement Density $\left(\mathrm{g} / \mathrm{cm}^{3}\right)$ Summary Statistics.

\begin{tabular}{|c|c|c|c|c|c|c|}
\hline $\begin{array}{l}\text { Combined } \\
\text { Specimens }\end{array}$ & Mean & Std Dev & $\mathrm{CoV}(\%)$ & Median & $\begin{array}{l}\text { Upper } \\
\text { Limit }\end{array}$ & $\begin{array}{l}\text { Lower } \\
\text { Limit }\end{array}$ \\
\hline IG-110 & 1.7677 & 0.0080 & 0.45 & 1.7689 & 1.7799 & 1.7565 \\
\hline NBG-17 & 1.8322 & 0.0076 & 0.42 & 1.8305 & 1.8453 & 1.8192 \\
\hline NBG-18 & 1.8525 & 0.0053 & 0.29 & 1.8520 & 1.8648 & 1.8401 \\
\hline PCEA & 1.7831 & 0.0085 & 0.47 & 1.7815 & 1.7959 & 1.7694 \\
\hline 2114 & 1.8167 & 0.0039 & 0.22 & 1.8170 & 1.8243 & 1.8074 \\
\hline $\begin{array}{c}\text { Against } \\
\text { Grain } \\
\text { Specimens }\end{array}$ & Mean & Std Dev & $\mathrm{CoV}(\%)$ & Median & $\begin{array}{l}\text { Upper } \\
\text { Limit }\end{array}$ & $\begin{array}{l}\text { Lower } \\
\text { Limit }\end{array}$ \\
\hline IG-110 & 1.7579 & 0.0090 & 0.51 & 1.7578 & 1.7688 & 1.7333 \\
\hline NBG-17 & 1.8347 & 0.0083 & 0.45 & 1.8368 & 1.8453 & 1.8192 \\
\hline NBG-18 & 1.8540 & 0.0053 & 0.28 & 1.8530 & 1.8674 & 1.8441 \\
\hline PCEA & 1.7927 & 0.0111 & 0.62 & 1.7893 & 1.8063 & 1.7806 \\
\hline 2114 & & & & & & \\
\hline $\begin{array}{l}\text { With Grain } \\
\text { Specimens }\end{array}$ & Mean & Std Dev & $\mathrm{CoV}(\%)$ & Median & $\begin{array}{l}\text { Upper } \\
\text { Limit }\end{array}$ & $\begin{array}{l}\text { Lower } \\
\text { Limit }\end{array}$ \\
\hline IG-110 & 1.7710 & 0.0039 & 0.22 & 1.7710 & 1.7799 & 1.7613 \\
\hline NBG-17 & 1.8281 & 0.0038 & 0.21 & 1.8269 & 1.8366 & 1.8216 \\
\hline NBG-18 & 1.8492 & 0.0036 & 0.20 & 1.8498 & 1.8566 & 1.8415 \\
\hline PCEA & 1.7798 & 0.0038 & 0.21 & 1.7800 & 1.7869 & 1.7698 \\
\hline 2114 & 1.8167 & 0.0039 & 0.22 & 1.8170 & 1.8243 & 1.8074 \\
\hline
\end{tabular}


Table B-5. Creep Coefficient of Thermal Expansion $(1 / \mathrm{K})$ at $100{ }^{\circ} \mathrm{C}$ Summary Statistics.

\begin{tabular}{|c|c|c|c|c|c|c|}
\hline $\begin{array}{l}\text { Combined } \\
\text { Specimens }\end{array}$ & Mean & Std Dev & $\operatorname{CoV}(\%)$ & Median & $\begin{array}{l}\text { Upper } \\
\text { Limit }\end{array}$ & $\begin{array}{l}\text { Lower } \\
\text { Limit }\end{array}$ \\
\hline IG-110 & $3.74 \mathrm{E}-06$ & $2.21 \mathrm{E}-07$ & 5.89 & $3.70 \mathrm{E}-06$ & $4.16 \mathrm{E}-06$ & $3.34 \mathrm{E}-06$ \\
\hline NBG-17 & 4.78E-06 & $9.03 \mathrm{E}-08$ & 1.89 & 4.79E-06 & $4.96 \mathrm{E}-06$ & $4.56 \mathrm{E}-06$ \\
\hline NBG-18 & $4.86 \mathrm{E}-06$ & 4.83E-07 & 9.94 & $4.82 \mathrm{E}-06$ & $5.84 \mathrm{E}-06$ & $3.97 \mathrm{E}-06$ \\
\hline PCEA & 4.13E-06 & $1.74 \mathrm{E}-07$ & 4.21 & 4.11E-06 & 4.52E-06 & $3.86 \mathrm{E}-06$ \\
\hline 2114 & 4.73E-06 & $1.18 \mathrm{E}-07$ & 2.49 & 4.74E-06 & 4.95E-06 & 4.49E-06 \\
\hline $\begin{array}{c}\text { Against } \\
\text { Grain } \\
\text { Specimens }\end{array}$ & Mean & Std Dev & $\operatorname{CoV}(\%)$ & Median & $\begin{array}{l}\text { Upper } \\
\text { Limit }\end{array}$ & $\begin{array}{c}\text { Lower } \\
\text { Limit }\end{array}$ \\
\hline IG-110 & $3.91 \mathrm{E}-06$ & $2.09 \mathrm{E}-07$ & 5.33 & $3.78 \mathrm{E}-06$ & $4.16 \mathrm{E}-06$ & $3.68 \mathrm{E}-06$ \\
\hline NBG-17 & $4.78 \mathrm{E}-06$ & $9.86 \mathrm{E}-08$ & 2.06 & 4.79E-06 & $4.96 \mathrm{E}-06$ & $4.53 \mathrm{E}-06$ \\
\hline NBG-18 & 4.82E-06 & 4.82E-07 & 10.00 & 4.77E-06 & $5.74 \mathrm{E}-06$ & 4.02E-06 \\
\hline PCEA & $4.38 \mathrm{E}-06$ & $8.78 \mathrm{E}-08$ & 2.00 & $4.39 \mathrm{E}-06$ & $4.53 \mathrm{E}-06$ & $4.25 \mathrm{E}-06$ \\
\hline 2114 & & & & & & \\
\hline $\begin{array}{l}\text { With Grain } \\
\text { Specimens }\end{array}$ & Mean & Std Dev & $\operatorname{CoV}(\%)$ & Median & $\begin{array}{l}\text { Upper } \\
\text { Limit } \\
\end{array}$ & $\begin{array}{c}\text { Lower } \\
\text { Limit } \\
\end{array}$ \\
\hline IG-110 & $3.68 \mathrm{E}-06$ & $1.94 \mathrm{E}-07$ & 5.28 & $3.66 \mathrm{E}-06$ & $4.15 \mathrm{E}-06$ & $3.34 \mathrm{E}-06$ \\
\hline NBG-17 & 4.77E-06 & $6.65 \mathrm{E}-08$ & 1.39 & 4.79E-06 & $4.86 \mathrm{E}-06$ & 4.63E-06 \\
\hline NBG-18 & $4.98 \mathrm{E}-06$ & $4.80 \mathrm{E}-07$ & 9.64 & $5.13 \mathrm{E}-06$ & $5.61 \mathrm{E}-06$ & $4.38 \mathrm{E}-06$ \\
\hline PCEA & 4.04E-06 & $9.20 \mathrm{E}-08$ & 2.27 & $4.05 \mathrm{E}-06$ & $4.21 \mathrm{E}-06$ & $3.86 \mathrm{E}-06$ \\
\hline 2114 & $4.73 \mathrm{E}-06$ & $1.18 \mathrm{E}-07$ & 2.49 & $4.74 \mathrm{E}-06$ & $4.95 \mathrm{E}-06$ & $4.49 \mathrm{E}-06$ \\
\hline
\end{tabular}


Table B-6. Creep Coefficient of Thermal Expansion $(1 / \mathrm{K})$ at $500{ }^{\circ} \mathrm{C}$ Summary Statistics.

\begin{tabular}{|c|c|c|c|c|c|c|}
\hline $\begin{array}{l}\text { Combined } \\
\text { Specimens }\end{array}$ & Mean & Std Dev & $\mathrm{CoV}(\%)$ & Median & $\begin{array}{l}\text { Upper } \\
\text { Limit }\end{array}$ & $\begin{array}{c}\text { Lower } \\
\text { Limit }\end{array}$ \\
\hline IG-110 & 4.07E-06 & $2.26 \mathrm{E}-07$ & 5.55 & $4.07 \mathrm{E}-06$ & $4.61 \mathrm{E}-06$ & $3.63 \mathrm{E}-06$ \\
\hline NBG-17 & 5.32E-06 & 8.73E-08 & 1.64 & $5.32 \mathrm{E}-06$ & $5.50 \mathrm{E}-06$ & $5.12 \mathrm{E}-06$ \\
\hline NBG-18 & 5.11E-06 & $1.33 \mathrm{E}-07$ & 2.61 & $5.09 \mathrm{E}-06$ & $5.36 \mathrm{E}-06$ & 4.74E-06 \\
\hline PCEA & 4.60E-06 & $1.66 \mathrm{E}-07$ & 3.61 & $4.53 \mathrm{E}-06$ & 4.99E-06 & 4.37E-06 \\
\hline 2114 & 5.23E-06 & $1.03 \mathrm{E}-07$ & 1.96 & $5.22 \mathrm{E}-06$ & 5.39E-06 & $5.06 \mathrm{E}-06$ \\
\hline $\begin{array}{c}\text { Against } \\
\text { Grain } \\
\text { Specimens }\end{array}$ & Mean & Std Dev & $\operatorname{CoV}(\%)$ & Median & $\begin{array}{l}\text { Upper } \\
\text { Limit }\end{array}$ & $\begin{array}{c}\text { Lower } \\
\text { Limit }\end{array}$ \\
\hline IG-110 & $3.80 \mathrm{E}-06$ & $9.67 \mathrm{E}-08$ & 2.54 & $3.80 \mathrm{E}-06$ & $3.96 \mathrm{E}-06$ & $3.63 \mathrm{E}-06$ \\
\hline NBG-17 & 5.32E-06 & $9.36 \mathrm{E}-08$ & 1.76 & $5.34 \mathrm{E}-06$ & $5.50 \mathrm{E}-06$ & $5.13 \mathrm{E}-06$ \\
\hline NBG-18 & $5.10 \mathrm{E}-06$ & $1.38 \mathrm{E}-07$ & 2.71 & $5.07 \mathrm{E}-06$ & $5.36 \mathrm{E}-06$ & $4.73 \mathrm{E}-06$ \\
\hline PCEA & 4.85E-06 & $8.05 \mathrm{E}-08$ & 1.66 & 4.82E-06 & 4.99E-06 & 4.74E-06 \\
\hline 2114 & & & & & & \\
\hline $\begin{array}{l}\text { With Grain } \\
\text { Specimens }\end{array}$ & Mean & Std Dev & $\operatorname{CoV}(\%)$ & Median & $\begin{array}{l}\text { Upper } \\
\text { Limit }\end{array}$ & $\begin{array}{c}\text { Lower } \\
\text { Limit }\end{array}$ \\
\hline IG-110 & 4.17E-06 & $1.72 \mathrm{E}-07$ & 4.13 & $4.18 \mathrm{E}-06$ & 4.61E-06 & $3.83 \mathrm{E}-06$ \\
\hline NBG-17 & 5.30E-06 & $6.87 \mathrm{E}-08$ & 1.30 & 5.31E-06 & $5.41 \mathrm{E}-06$ & $5.17 \mathrm{E}-06$ \\
\hline NBG-18 & $5.12 \mathrm{E}-06$ & $1.16 \mathrm{E}-07$ & 2.27 & $5.15 \mathrm{E}-06$ & $5.32 \mathrm{E}-06$ & $4.91 \mathrm{E}-06$ \\
\hline PCEA & 4.52E-06 & 7.32E-08 & 1.62 & $4.50 \mathrm{E}-06$ & $4.68 \mathrm{E}-06$ & $4.37 \mathrm{E}-06$ \\
\hline 2114 & $5.23 \mathrm{E}-06$ & $1.03 \mathrm{E}-07$ & 1.96 & $5.22 \mathrm{E}-06$ & $5.39 \mathrm{E}-06$ & $5.06 \mathrm{E}-06$ \\
\hline
\end{tabular}


Table B-7. Creep Coefficient of Thermal Expansion (1/K) at $900{ }^{\circ} \mathrm{C}$ Summary Statistics.

\begin{tabular}{|c|c|c|c|c|c|c|}
\hline $\begin{array}{l}\text { Combined } \\
\text { Specimens }\end{array}$ & Mean & Std Dev & $\mathrm{CoV}(\%)$ & Median & $\begin{array}{l}\text { Upper } \\
\text { Limit }\end{array}$ & $\begin{array}{l}\text { Lower } \\
\text { Limit }\end{array}$ \\
\hline IG-110 & 4.37E-06 & $3.24 \mathrm{E}-07$ & 7.41 & 4.44E-06 & $4.89 \mathrm{E}-06$ & $3.76 \mathrm{E}-06$ \\
\hline NBG-17 & 5.74E-06 & $8.56 \mathrm{E}-08$ & 1.49 & $5.74 \mathrm{E}-06$ & 5.91E-06 & $5.57 \mathrm{E}-06$ \\
\hline NBG-18 & 5.31E-06 & $2.00 \mathrm{E}-07$ & 3.77 & $5.24 \mathrm{E}-06$ & $5.75 \mathrm{E}-06$ & $4.96 \mathrm{E}-06$ \\
\hline PCEA & $5.02 \mathrm{E}-06$ & $1.59 \mathrm{E}-07$ & 3.17 & $4.96 \mathrm{E}-06$ & $5.40 \mathrm{E}-06$ & $4.76 \mathrm{E}-06$ \\
\hline 2114 & 5.62E-06 & $9.44 \mathrm{E}-08$ & 1.68 & $5.61 \mathrm{E}-06$ & $5.83 \mathrm{E}-06$ & $5.40 \mathrm{E}-06$ \\
\hline $\begin{array}{c}\text { Against } \\
\text { Grain } \\
\text { Specimens }\end{array}$ & Mean & Std Dev & $\operatorname{CoV}(\%)$ & Median & $\begin{array}{l}\text { Upper } \\
\text { Limit }\end{array}$ & $\begin{array}{c}\text { Lower } \\
\text { Limit }\end{array}$ \\
\hline IG-110 & $3.90 \mathrm{E}-06$ & 8.71E-08 & 2.23 & $3.91 \mathrm{E}-06$ & $4.03 \mathrm{E}-06$ & $3.76 \mathrm{E}-06$ \\
\hline NBG-17 & 5.74E-06 & $9.29 \mathrm{E}-08$ & 1.62 & $5.75 \mathrm{E}-06$ & 5.91E-06 & $5.57 \mathrm{E}-06$ \\
\hline NBG-18 & 5.32E-06 & $2.18 \mathrm{E}-07$ & 4.10 & $5.24 \mathrm{E}-06$ & $5.75 \mathrm{E}-06$ & $5.01 \mathrm{E}-06$ \\
\hline PCEA & $5.25 \mathrm{E}-06$ & 9.99E-08 & 1.90 & $5.24 \mathrm{E}-06$ & $5.40 \mathrm{E}-06$ & $5.12 \mathrm{E}-06$ \\
\hline 2114 & & & & & & \\
\hline $\begin{array}{l}\text { With Grain } \\
\text { Specimens }\end{array}$ & Mean & Std Dev & $\mathrm{CoV}(\%)$ & Median & $\begin{array}{l}\text { Upper } \\
\text { Limit }\end{array}$ & $\begin{array}{c}\text { Lower } \\
\text { Limit }\end{array}$ \\
\hline IG-110 & 4.54E-06 & $1.68 \mathrm{E}-07$ & 3.69 & 4.57E-06 & $4.89 \mathrm{E}-06$ & $4.24 \mathrm{E}-06$ \\
\hline NBG-17 & 5.72E-06 & $6.25 \mathrm{E}-08$ & 1.09 & $5.72 \mathrm{E}-06$ & $5.82 \mathrm{E}-06$ & $5.57 \mathrm{E}-06$ \\
\hline NBG-18 & $5.27 \mathrm{E}-06$ & $1.12 \mathrm{E}-07$ & 2.12 & $5.27 \mathrm{E}-06$ & $5.45 \mathrm{E}-06$ & $5.04 \mathrm{E}-06$ \\
\hline PCEA & 4.94E-06 & 7.35E-08 & 1.49 & $4.92 \mathrm{E}-06$ & $5.10 \mathrm{E}-06$ & $4.76 \mathrm{E}-06$ \\
\hline 2114 & $5.62 \mathrm{E}-06$ & $9.44 \mathrm{E}-08$ & 1.68 & 5.61E-06 & $5.83 \mathrm{E}-06$ & $5.40 \mathrm{E}-06$ \\
\hline
\end{tabular}


Table B-8. Creep Modulus (GPa) by Sonic Resonance Summary Statistics.

\begin{tabular}{|c|c|c|c|c|c|c|}
\hline $\begin{array}{l}\text { Combined } \\
\text { Specimens }\end{array}$ & Mean & Std Dev & $\operatorname{CoV}(\%)$ & Median & $\begin{array}{l}\text { Upper } \\
\text { Limit }\end{array}$ & $\begin{array}{l}\text { Lower } \\
\text { Limit }\end{array}$ \\
\hline IG-110 & 9.3956 & 0.3429 & 3.65 & 9.4030 & 10.0089 & 8.8158 \\
\hline NBG-17 & 11.6980 & 0.3630 & 3.10 & 11.7000 & 12.3000 & 11.0000 \\
\hline NBG-18 & 12.2350 & 0.2843 & 2.32 & 12.2598 & 12.9371 & 11.6194 \\
\hline PCEA & 9.2313 & 0.4368 & 4.73 & 9.2000 & 10.5000 & 8.3000 \\
\hline 2114 & 10.5620 & 0.2101 & 1.99 & 10.5000 & 10.9000 & 10.1000 \\
\hline $\begin{array}{c}\text { Against } \\
\text { Grain } \\
\text { Specimens }\end{array}$ & Mean & Std Dev & $\mathrm{CoV}(\%)$ & Median & $\begin{array}{c}\text { Upper } \\
\text { Limit }\end{array}$ & $\begin{array}{r}\text { Lower } \\
\text { Limit }\end{array}$ \\
\hline IG-110 & 9.7232 & 0.2456 & 2.53 & 9.7869 & 10.0089 & 9.2316 \\
\hline NBG-17 & 11.7421 & 0.4084 & 3.48 & 11.8000 & 12.3000 & 11.0000 \\
\hline NBG-18 & 12.3538 & 0.1873 & 1.52 & 12.3455 & 12.8567 & 12.0781 \\
\hline PCEA & 9.8050 & 0.2305 & 2.35 & 9.8000 & 9.9500 & 9.5500 \\
\hline 2114 & & & & & & \\
\hline $\begin{array}{l}\text { With Grain } \\
\text { Specimens }\end{array}$ & Mean & Std Dev & $\mathrm{CoV}(\%)$ & Median & $\begin{array}{c}\text { Upper } \\
\text { Limit }\end{array}$ & $\begin{array}{r}\text { Lower } \\
\text { Limit } \\
\end{array}$ \\
\hline IG-110 & 9.2773 & 0.2938 & 3.17 & 9.2876 & 9.8716 & 8.8158 \\
\hline NBG-17 & 11.5692 & 0.1032 & 0.89 & 11.6000 & 11.7500 & 11.4000 \\
\hline NBG-18 & 11.7996 & 0.0674 & 0.57 & 11.7841 & 11.8986 & 11.6411 \\
\hline PCEA & 9.0400 & 0.2993 & 3.31 & 9.0500 & 9.7000 & 8.3000 \\
\hline 2114 & 10.5620 & 0.2101 & 1.99 & 10.5000 & 10.9000 & 10.1000 \\
\hline
\end{tabular}


Table B-9. Creep Resistivity $(\mu \Omega-m)$ Summary Statistics.

\begin{tabular}{|c|c|c|c|c|c|c|}
\hline $\begin{array}{l}\text { Combined } \\
\text { Specimens }\end{array}$ & Mean & Std Dev & $\mathrm{CoV}(\%)$ & Median & $\begin{array}{l}\text { Upper } \\
\text { Limit }\end{array}$ & $\begin{array}{l}\text { Lower } \\
\text { Limit }\end{array}$ \\
\hline IG-110 & 10.6107 & 0.3461 & 3.26 & 10.6494 & 11.2873 & 9.7903 \\
\hline NBG-17 & 11.0556 & 0.3253 & 2.94 & 11.1012 & 11.5616 & 10.4809 \\
\hline NBG-18 & 9.9304 & 0.2935 & 2.96 & 9.8590 & 10.5703 & 9.3319 \\
\hline PCEA & 8.5234 & 0.1608 & 1.89 & 8.5200 & 8.8417 & 8.2006 \\
\hline 2114 & 12.5698 & 0.3780 & 3.01 & 12.5383 & 13.3510 & 11.8955 \\
\hline $\begin{array}{c}\text { Against } \\
\text { Grain } \\
\text { Specimens }\end{array}$ & Mean & Std Dev & $\operatorname{CoV}(\%)$ & Median & $\begin{array}{l}\text { Upper } \\
\text { Limit }\end{array}$ & $\begin{array}{r}\text { Lower } \\
\text { Limit }\end{array}$ \\
\hline IG-110 & 10.8653 & 0.2473 & 2.28 & 10.7948 & 11.2873 & 10.4669 \\
\hline NBG-17 & 11.0184 & 0.3247 & 2.95 & 11.0966 & 11.5441 & 10.5443 \\
\hline NBG-18 & 9.8529 & 0.2569 & 2.61 & 9.7938 & 10.5456 & 9.3319 \\
\hline PCEA & 8.6201 & 0.1455 & 1.69 & 8.6123 & 8.8417 & 8.3332 \\
\hline 2114 & & & & & & \\
\hline $\begin{array}{l}\text { With Grain } \\
\text { Specimens }\end{array}$ & Mean & Std Dev & $\operatorname{CoV}(\%)$ & Median & $\begin{array}{c}\text { Upper } \\
\text { Limit }\end{array}$ & $\begin{array}{r}\text { Lower } \\
\text { Limit } \\
\end{array}$ \\
\hline IG-110 & 10.5355 & 0.3370 & 3.20 & 10.5253 & 11.1588 & 9.7490 \\
\hline NBG-17 & 11.1512 & 0.3169 & 2.84 & 11.1768 & 11.5616 & 10.5423 \\
\hline NBG-18 & 10.2146 & 0.2448 & 2.40 & 10.2279 & 10.5703 & 9.7962 \\
\hline PCEA & 8.4762 & 0.1476 & 1.74 & 8.4637 & 8.7502 & 8.2006 \\
\hline 2114 & 12.5698 & 0.3780 & 3.01 & 12.5383 & 13.3510 & 11.8955 \\
\hline
\end{tabular}


Table B-10. Creep Young's Modulus (GPa) by Sonic Velocity.

\begin{tabular}{|c|c|c|c|c|c|c|}
\hline $\begin{array}{l}\text { Combined } \\
\text { Specimens }\end{array}$ & Mean & Std Dev & $\operatorname{CoV}(\%)$ & Median & $\begin{array}{l}\text { Upper } \\
\text { Limit }\end{array}$ & $\begin{array}{c}\text { Lower } \\
\text { Limit }\end{array}$ \\
\hline IG-110 & 10.3387 & 0.4506 & 4.36 & 10.3762 & 11.1839 & 9.5917 \\
\hline NBG-17 & 15.0395 & 0.5215 & 3.47 & 15.0492 & 15.9677 & 13.8967 \\
\hline NBG-18 & 15.9385 & 0.3399 & 2.13 & 15.9665 & 16.7087 & 15.1342 \\
\hline PCEA & 12.2545 & 0.2194 & 1.79 & 12.2684 & 12.7188 & 11.7656 \\
\hline 2114 & 12.1019 & 0.3120 & 2.58 & 12.0945 & 12.6568 & 11.3583 \\
\hline $\begin{array}{c}\text { Against } \\
\text { Grain } \\
\text { Specimens }\end{array}$ & Mean & Std Dev & $\mathrm{CoV}(\%)$ & Median & $\begin{array}{c}\text { Upper } \\
\text { Limit }\end{array}$ & $\begin{array}{r}\text { Lower } \\
\text { Limit }\end{array}$ \\
\hline IG-110 & 10.8949 & 0.2339 & 2.15 & 10.9756 & 11.1839 & 10.4555 \\
\hline NBG-17 & 15.0319 & 0.6019 & 4.00 & 15.0417 & 15.9677 & 13.8967 \\
\hline NBG-18 & 16.0524 & 0.2793 & 1.74 & 16.0800 & 16.7087 & 15.3682 \\
\hline PCEA & 12.1252 & 0.1977 & 1.63 & 12.1041 & 12.4930 & 11.7656 \\
\hline 2114 & & & & & & \\
\hline $\begin{array}{l}\text { With Grain } \\
\text { Specimens }\end{array}$ & Mean & Std Dev & $\mathrm{CoV}(\%)$ & Median & $\begin{array}{l}\text { Upper } \\
\text { Limit }\end{array}$ & $\begin{array}{r}\text { Lower } \\
\text { Limit } \\
\end{array}$ \\
\hline IG-110 & 10.1744 & 0.3578 & 3.52 & 10.2129 & 11.0391 & 9.5917 \\
\hline NBG-17 & 15.0618 & 0.1241 & 0.82 & 15.0572 & 15.3258 & 14.9050 \\
\hline NBG-18 & 15.5212 & 0.1759 & 1.13 & 15.4690 & 15.8401 & 15.3056 \\
\hline PCEA & 12.2976 & 0.2105 & 1.71 & 12.2961 & 12.7188 & 11.7963 \\
\hline 2114 & 12.1019 & 0.3120 & 2.58 & 12.0945 & 12.6568 & 11.3583 \\
\hline
\end{tabular}


Table B-11. Creep Shear Modulus (GPa) by Sonic Velocity.

\begin{tabular}{|c|c|c|c|c|c|c|}
\hline $\begin{array}{l}\text { Combined } \\
\text { Specimens }\end{array}$ & Mean & Std Dev & $\operatorname{CoV}(\%)$ & Median & $\begin{array}{l}\text { Upper } \\
\text { Limit } \\
\end{array}$ & $\begin{array}{c}\text { Lower } \\
\text { Limit } \\
\end{array}$ \\
\hline IG-110 & 4.2772 & 0.1356 & 3.17 & 4.2817 & 4.5574 & 3.9963 \\
\hline NBG-17 & 5.0064 & 0.1163 & 2.32 & 5.0030 & 5.2050 & 4.8001 \\
\hline NBG-18 & 5.2018 & 0.0774 & 1.49 & 5.1916 & 5.3731 & 5.0536 \\
\hline PCEA & 4.4711 & 0.0711 & 1.59 & 4.4743 & 4.6597 & 4.2819 \\
\hline 2114 & 4.4124 & 0.0631 & 1.43 & 4.4303 & 4.5688 & 4.2577 \\
\hline $\begin{array}{c}\text { Against } \\
\text { Grain } \\
\text { Specimens }\end{array}$ & Mean & Std Dev & $\mathrm{CoV}(\%)$ & Median & $\begin{array}{l}\text { Upper } \\
\text { Limit }\end{array}$ & $\begin{array}{c}\text { Lower } \\
\text { Limit }\end{array}$ \\
\hline IG-110 & 4.3717 & 0.0903 & 2.07 & 4.3339 & 4.5574 & 4.2623 \\
\hline NBG-17 & 5.0307 & 0.1232 & 2.45 & 5.0770 & 5.2050 & 4.8001 \\
\hline NBG-18 & 5.2286 & 0.0632 & 1.21 & 5.2203 & 5.3731 & 5.1300 \\
\hline PCEA & 4.4641 & 0.1033 & 2.31 & 4.4580 & 4.6752 & 4.3299 \\
\hline 2114 & & & & & & \\
\hline $\begin{array}{l}\text { With Grain } \\
\text { Specimens }\end{array}$ & Mean & Std Dev & $\operatorname{CoV}(\%)$ & Median & $\begin{array}{l}\text { Upper } \\
\text { Limit }\end{array}$ & $\begin{array}{c}\text { Lower } \\
\text { Limit }\end{array}$ \\
\hline IG-110 & 4.2493 & 0.1348 & 3.17 & 4.2346 & 4.5304 & 3.9963 \\
\hline NBG-17 & 4.9354 & 0.0470 & 0.95 & 4.9458 & 5.0072 & 4.8570 \\
\hline NBG-18 & 5.1034 & 0.0294 & 0.58 & 5.1131 & 5.1407 & 5.0536 \\
\hline PCEA & 4.4734 & 0.0575 & 1.28 & 4.4744 & 4.5889 & 4.3342 \\
\hline 2114 & 4.4124 & 0.0631 & 1.43 & 4.4303 & 4.5688 & 4.2577 \\
\hline
\end{tabular}


Table B-12. Piggyback Pre Thermal Measurement Length (mm) Summary Statistics.

\begin{tabular}{|c|c|c|c|c|c|c|}
\hline $\begin{array}{l}\text { Combined } \\
\text { Specimens }\end{array}$ & Mean & Std Dev & $\operatorname{CoV}(\%)$ & Median & $\begin{array}{l}\text { Upper } \\
\text { Limit }\end{array}$ & $\begin{array}{c}\text { Lower } \\
\text { Limit }\end{array}$ \\
\hline IG-110 & 6.2725 & 0.0266 & 0.42 & 6.2725 & 6.3120 & 6.2345 \\
\hline NBG-17 & 6.3144 & 0.0074 & 0.12 & 6.3138 & 6.3224 & 6.3049 \\
\hline NBG-18 & 6.2623 & 0.1893 & 3.02 & 6.3198 & 6.3370 & 6.2648 \\
\hline PCEA & 6.3252 & 0.0302 & 0.48 & 6.3310 & 6.3573 & 6.3014 \\
\hline 2114 & 6.3270 & 0.0059 & 0.09 & 6.3276 & 6.3378 & 6.3115 \\
\hline $\begin{array}{c}\text { Against } \\
\text { Grain } \\
\text { Specimens }\end{array}$ & Mean & Std Dev & $\operatorname{CoV}(\%)$ & Median & $\begin{array}{c}\text { Upper } \\
\text { Limit }\end{array}$ & $\begin{array}{c}\text { Lower } \\
\text { Limit }\end{array}$ \\
\hline IG-110 & 6.2735 & 0.0364 & 0.58 & 6.2799 & 6.3410 & 6.2078 \\
\hline NBG-17 & 6.3141 & 0.0081 & 0.13 & 6.3129 & 6.3232 & 6.3037 \\
\hline NBG-18 & 6.2910 & 0.1346 & 2.14 & 6.3168 & 6.3358 & 6.2774 \\
\hline PCEA & 6.3324 & 0.0224 & 0.35 & 6.3381 & 6.3533 & 6.3198 \\
\hline 2114 & & & & & & \\
\hline $\begin{array}{l}\text { With Grain } \\
\text { Specimens }\end{array}$ & Mean & Std Dev & $\operatorname{CoV}(\%)$ & Median & $\begin{array}{c}\text { Upper } \\
\text { Limit }\end{array}$ & $\begin{array}{c}\text { Lower } \\
\text { Limit }\end{array}$ \\
\hline IG-110 & 6.2715 & 0.0097 & 0.15 & 6.2695 & 6.2938 & 6.2491 \\
\hline NBG-17 & 6.3151 & 0.0057 & 0.09 & 6.3149 & 6.3196 & 6.3096 \\
\hline NBG-18 & 6.2048 & 0.2613 & 4.21 & 6.3230 & 6.3370 & 6.2385 \\
\hline PCEA & 6.3186 & 0.0349 & 0.55 & 6.3265 & 6.3469 & 6.3044 \\
\hline 2114 & 6.3270 & 0.0059 & 0.09 & 6.3276 & 6.3378 & 6.3115 \\
\hline
\end{tabular}


Table B-13. Piggyback Pre Thermal Measurement Diameter (mm) Summary Statistics.

\begin{tabular}{|c|c|c|c|c|c|c|}
\hline $\begin{array}{l}\text { Combined } \\
\text { Specimens }\end{array}$ & Mean & Std Dev & $\operatorname{CoV}(\%)$ & Median & $\begin{array}{l}\text { Upper } \\
\text { Limit } \\
\end{array}$ & $\begin{array}{c}\text { Lower } \\
\text { Limit }\end{array}$ \\
\hline IG-110 & 12.4695 & 0.0021 & 0.02 & 12.4695 & 12.4735 & 12.4650 \\
\hline NBG-17 & 12.4599 & 0.0041 & 0.03 & 12.4583 & 12.4650 & 12.4560 \\
\hline NBG-18 & 12.4874 & 0.0104 & 0.08 & 12.4865 & 12.5006 & 12.4756 \\
\hline PCEA & 12.4754 & 0.0129 & 0.10 & 12.4765 & 12.4983 & 12.4523 \\
\hline 2114 & 12.4737 & 0.0038 & 0.03 & 12.4740 & 12.4815 & 12.4660 \\
\hline $\begin{array}{c}\text { Against } \\
\text { Grain } \\
\text { Specimens }\end{array}$ & Mean & Std Dev & $\operatorname{CoV}(\%)$ & Median & $\begin{array}{l}\text { Upper } \\
\text { Limit }\end{array}$ & $\begin{array}{c}\text { Lower } \\
\text { Limit }\end{array}$ \\
\hline IG-110 & 12.4703 & 0.0022 & 0.02 & 12.4710 & 12.4735 & 12.4658 \\
\hline NBG-17 & 12.4590 & 0.0040 & 0.03 & 12.4580 & 12.4633 & 12.4560 \\
\hline NBG-18 & 12.4901 & 0.0053 & 0.04 & 12.4883 & 12.5015 & 12.4835 \\
\hline PCEA & 12.4724 & 0.0078 & 0.06 & 12.4700 & 12.4766 & 12.4660 \\
\hline 2114 & & & & & & \\
\hline $\begin{array}{l}\text { With Grain } \\
\text { Specimens }\end{array}$ & Mean & Std Dev & $\mathrm{CoV}(\%)$ & Median & $\begin{array}{c}\text { Upper } \\
\text { Limit }\end{array}$ & $\begin{array}{c}\text { Lower } \\
\text { Limit } \\
\end{array}$ \\
\hline IG-110 & 12.4687 & 0.0016 & 0.01 & 12.4690 & 12.4725 & 12.4655 \\
\hline NBG-17 & 12.4615 & 0.0039 & 0.03 & 12.4595 & 12.4690 & 12.4570 \\
\hline NBG-18 & 12.4820 & 0.0152 & 0.12 & 12.4850 & 12.4880 & 12.4794 \\
\hline PCEA & 12.4781 & 0.0159 & 0.13 & 12.4805 & 12.4885 & 12.4725 \\
\hline 2114 & 12.4737 & 0.0038 & 0.03 & 12.4740 & 12.4815 & 12.4660 \\
\hline
\end{tabular}


Table B-14. Piggyback Pre Thermal Measurement Mass (g) Summary Statistics.

\begin{tabular}{|c|c|c|c|c|c|c|}
\hline $\begin{array}{l}\text { Combined } \\
\text { Specimens }\end{array}$ & Mean & Std Dev & $\mathrm{CoV}(\%)$ & Median & $\begin{array}{l}\text { Upper } \\
\text { Limit }\end{array}$ & $\begin{array}{c}\text { Lower } \\
\text { Limit }\end{array}$ \\
\hline IG-110 & 1.3518 & 0.0079 & 0.59 & 1.3542 & 1.3648 & 1.3360 \\
\hline NBG-17 & 1.4085 & 0.0051 & 0.36 & 1.4095 & 1.4160 & 1.3975 \\
\hline NBG-18 & 1.4193 & 0.0439 & 3.09 & 1.4320 & 1.4455 & 1.4143 \\
\hline PCEA & 1.3763 & 0.0127 & 0.92 & 1.3766 & 1.3912 & 1.3629 \\
\hline 2114 & 1.4083 & 0.0047 & 0.33 & 1.4086 & 1.4138 & 1.4023 \\
\hline $\begin{array}{c}\text { Against } \\
\text { Grain } \\
\text { Specimens }\end{array}$ & Mean & Std Dev & $\mathrm{CoV}(\%)$ & Median & $\begin{array}{l}\text { Upper } \\
\text { Limit }\end{array}$ & $\begin{array}{c}\text { Lower } \\
\text { Limit }\end{array}$ \\
\hline IG-110 & 1.3477 & 0.0087 & 0.65 & 1.3511 & 1.3583 & 1.3237 \\
\hline NBG-17 & 1.4081 & 0.0055 & 0.39 & 1.4090 & 1.4160 & 1.3972 \\
\hline NBG-18 & 1.4269 & 0.0316 & 2.21 & 1.4328 & 1.4455 & 1.4149 \\
\hline PCEA & 1.3805 & 0.0132 & 0.96 & 1.3787 & 1.3980 & 1.3616 \\
\hline 2114 & & & & & & \\
\hline $\begin{array}{l}\text { With Grain } \\
\text { Specimens }\end{array}$ & Mean & Std Dev & $\operatorname{CoV}(\%)$ & Median & $\begin{array}{l}\text { Upper } \\
\text { Limit }\end{array}$ & $\begin{array}{c}\text { Lower } \\
\text { Limit }\end{array}$ \\
\hline IG-110 & 1.3559 & 0.0040 & 0.30 & 1.3561 & 1.3627 & 1.3492 \\
\hline NBG-17 & 1.4093 & 0.0039 & 0.28 & 1.4103 & 1.4160 & 1.4018 \\
\hline NBG-18 & 1.4040 & 0.0595 & 4.24 & 1.4315 & 1.4382 & 1.3791 \\
\hline PCEA & 1.3724 & 0.0109 & 0.80 & 1.3762 & 1.3858 & 1.3535 \\
\hline 2114 & 1.4083 & 0.0047 & 0.33 & 1.4086 & 1.4138 & 1.4023 \\
\hline
\end{tabular}


Table B-15. Piggyback Pre Thermal Measurement Density $\left(\mathrm{g} / \mathrm{cm}^{3}\right)$ Summary Statistics.

\begin{tabular}{|c|c|c|c|c|c|c|}
\hline $\begin{array}{l}\text { Combined } \\
\text { Specimens }\end{array}$ & Mean & Std Dev & $\operatorname{CoV}(\%)$ & Median & $\begin{array}{l}\text { Upper } \\
\text { Limit } \\
\end{array}$ & $\begin{array}{c}\text { Lower } \\
\text { Limit } \\
\end{array}$ \\
\hline IG-110 & 1.7648 & 0.0108 & 0.61 & 1.7682 & 1.7819 & 1.7432 \\
\hline NBG-17 & 1.8294 & 0.0066 & 0.36 & 1.8310 & 1.8405 & 1.8143 \\
\hline NBG-18 & 1.8505 & 0.0059 & 0.32 & 1.8494 & 1.8643 & 1.8392 \\
\hline PCEA & 1.7801 & 0.0114 & 0.64 & 1.7793 & 1.7982 & 1.7594 \\
\hline 2114 & 1.8214 & 0.0058 & 0.32 & 1.8221 & 1.8277 & 1.8143 \\
\hline $\begin{array}{c}\text { Against } \\
\text { Grain } \\
\text { Specimens }\end{array}$ & Mean & Std Dev & $\operatorname{CoV}(\%)$ & Median & $\begin{array}{l}\text { Upper } \\
\text { Limit }\end{array}$ & $\begin{array}{c}\text { Lower } \\
\text { Limit }\end{array}$ \\
\hline IG-110 & 1.7590 & 0.0118 & 0.67 & 1.7642 & 1.7742 & 1.7311 \\
\hline NBG-17 & 1.8292 & 0.0075 & 0.41 & 1.8306 & 1.8405 & 1.8128 \\
\hline NBG-18 & 1.8512 & 0.0063 & 0.34 & 1.8498 & 1.8637 & 1.8392 \\
\hline PCEA & 1.7843 & 0.0136 & 0.76 & 1.7818 & 1.8030 & 1.7624 \\
\hline 2114 & & & & & & \\
\hline $\begin{array}{l}\text { With Grain } \\
\text { Specimens }\end{array}$ & Mean & Std Dev & $\mathrm{CoV}(\%)$ & Median & $\begin{array}{l}\text { Upper } \\
\text { Limit }\end{array}$ & $\begin{array}{r}\text { Lower } \\
\text { Limit } \\
\end{array}$ \\
\hline IG-110 & 1.7707 & 0.0051 & 0.29 & 1.7717 & 1.7819 & 1.7598 \\
\hline NBG-17 & 1.8297 & 0.0042 & 0.23 & 1.8310 & 1.8373 & 1.8222 \\
\hline NBG-18 & 1.8491 & 0.0048 & 0.26 & 1.8488 & 1.8572 & 1.8400 \\
\hline PCEA & 1.7762 & 0.0072 & 0.40 & 1.7778 & 1.7859 & 1.7576 \\
\hline 2114 & 1.8214 & 0.0058 & 0.32 & 1.8221 & 1.8277 & 1.8143 \\
\hline
\end{tabular}


Table B-16. Piggyback Diffusivity $\left(\mathrm{mm}^{2} / \mathrm{sec}\right)$ at $100{ }^{\circ} \mathrm{C}$ Summary Statistics.

\begin{tabular}{|c|c|c|c|c|c|c|}
\hline $\begin{array}{l}\text { Combined } \\
\text { Specimens }\end{array}$ & Mean & Std Dev & $\operatorname{CoV}(\%)$ & Median & $\begin{array}{l}\text { Upper } \\
\text { Limit }\end{array}$ & $\begin{array}{c}\text { Lower } \\
\text { Limit }\end{array}$ \\
\hline IG-110 & 78.0976 & 3.2416 & 4.15 & 77.9398 & 86.0053 & 72.6133 \\
\hline NBG-17 & 71.9314 & 1.2920 & 1.80 & 71.6307 & 73.9233 & 69.3947 \\
\hline NBG-18 & 77.6334 & 1.3433 & 1.73 & 77.9157 & 80.2463 & 75.1067 \\
\hline PCEA & 90.4238 & 1.6616 & 1.84 & 90.3427 & 93.7553 & 86.7930 \\
\hline 2114 & 64.5020 & 1.0857 & 1.68 & 64.8667 & 65.8610 & 62.2524 \\
\hline $\begin{array}{c}\text { Against } \\
\text { Grain } \\
\text { Specimens }\end{array}$ & Mean & Std Dev & $\mathrm{CoV}(\%)$ & Median & $\begin{array}{c}\text { Upper } \\
\text { Limit }\end{array}$ & $\begin{array}{r}\text { Lower } \\
\text { Limit }\end{array}$ \\
\hline IG-110 & 80.6697 & 2.5181 & 3.12 & 80.2780 & 86.0053 & 76.7370 \\
\hline NBG-17 & 72.0394 & 1.5837 & 2.20 & 72.2860 & 73.9233 & 69.3947 \\
\hline NBG-18 & 78.5665 & 0.7260 & 0.92 & 78.4175 & 80.2463 & 77.5300 \\
\hline PCEA & 89.6268 & 1.0166 & 1.13 & 89.5607 & 91.2883 & 87.4888 \\
\hline 2114 & & & & & & \\
\hline $\begin{array}{l}\text { With Grain } \\
\text { Specimens }\end{array}$ & Mean & Std Dev & $\operatorname{CoV}(\%)$ & Median & $\begin{array}{c}\text { Upper } \\
\text { Limit }\end{array}$ & $\begin{array}{r}\text { Lower } \\
\text { Limit } \\
\end{array}$ \\
\hline IG-110 & 75.9369 & 1.9408 & 2.56 & 75.5607 & 79.9697 & 72.6133 \\
\hline NBG-17 & 71.7771 & 0.7026 & 0.98 & 71.6307 & 73.0123 & 70.5500 \\
\hline NBG-18 & 76.1818 & 0.5232 & 0.69 & 76.2593 & 77.1370 & 75.1067 \\
\hline PCEA & 91.2207 & 1.8099 & 1.98 & 91.1330 & 93.7553 & 86.7930 \\
\hline 2114 & 64.5020 & 1.0857 & 1.68 & 64.8667 & 65.8610 & 62.2524 \\
\hline
\end{tabular}


Table B-17. Piggyback Diffusivity $\left(\mathrm{mm}^{2} / \mathrm{sec}\right)$ at $500{ }^{\circ} \mathrm{C}$ Summary Statistics.

\begin{tabular}{|c|c|c|c|c|c|c|}
\hline $\begin{array}{l}\text { Combined } \\
\text { Specimens }\end{array}$ & Mean & Std Dev & $\operatorname{CoV}(\%)$ & Median & $\begin{array}{l}\text { Upper } \\
\text { Limit }\end{array}$ & $\begin{array}{c}\text { Lower } \\
\text { Limit }\end{array}$ \\
\hline IG-110 & 29.2928 & 1.1749 & 4.01 & 29.1888 & 32.0940 & 27.3377 \\
\hline NBG-17 & 28.4710 & 0.5208 & 1.83 & 28.5293 & 29.2613 & 27.4027 \\
\hline NBG-18 & 29.8382 & 0.4790 & 1.61 & 29.9617 & 30.7150 & 28.9197 \\
\hline PCEA & 33.2679 & 0.6050 & 1.82 & 33.2430 & 34.6077 & 31.8770 \\
\hline 2114 & 26.8351 & 0.2722 & 1.01 & 26.9277 & 27.2227 & 26.3432 \\
\hline $\begin{array}{c}\text { Against } \\
\text { Grain } \\
\text { Specimens }\end{array}$ & Mean & Std Dev & $\operatorname{CoV}(\%)$ & Median & $\begin{array}{c}\text { Upper } \\
\text { Limit }\end{array}$ & $\begin{array}{r}\text { Lower } \\
\text { Limit }\end{array}$ \\
\hline IG-110 & 30.2169 & 0.9353 & 3.10 & 30.0943 & 32.0940 & 28.6987 \\
\hline NBG-17 & 28.4364 & 0.6309 & 2.22 & 28.5515 & 29.2613 & 27.4027 \\
\hline NBG-18 & 30.1731 & 0.2500 & 0.83 & 30.0803 & 30.6723 & 29.8673 \\
\hline PCEA & 33.0569 & 0.3464 & 1.05 & 33.1177 & 33.6613 & 32.2627 \\
\hline 2114 & & & & & & \\
\hline $\begin{array}{l}\text { With Grain } \\
\text { Specimens }\end{array}$ & Mean & Std Dev & $\operatorname{CoV}(\%)$ & Median & $\begin{array}{c}\text { Upper } \\
\text { Limit }\end{array}$ & $\begin{array}{r}\text { Lower } \\
\text { Limit } \\
\end{array}$ \\
\hline IG-110 & 28.5166 & 0.6959 & 2.44 & 28.4807 & 29.9997 & 27.3377 \\
\hline NBG-17 & 28.5204 & 0.3106 & 1.09 & 28.5293 & 28.9703 & 27.7907 \\
\hline NBG-18 & 29.3171 & 0.1894 & 0.65 & 29.3222 & 29.6327 & 28.9385 \\
\hline PCEA & 33.4789 & 0.7306 & 2.18 & 33.4663 & 34.6077 & 31.7110 \\
\hline 2114 & 26.8351 & 0.2722 & 1.01 & 26.9277 & 27.2227 & 26.3432 \\
\hline
\end{tabular}


Table B-18. Piggyback Diffusivity $\left(\mathrm{mm}^{2} / \mathrm{sec}\right)$ at $900{ }^{\circ} \mathrm{C}$ Summary Statistics.

\begin{tabular}{|c|c|c|c|c|c|c|}
\hline $\begin{array}{l}\text { Combined } \\
\text { Specimens }\end{array}$ & Mean & Std Dev & $\operatorname{CoV}(\%)$ & Median & $\begin{array}{l}\text { Upper } \\
\text { Limit }\end{array}$ & $\begin{array}{c}\text { Lower } \\
\text { Limit }\end{array}$ \\
\hline IG-110 & 18.6745 & 0.7155 & 3.83 & 18.6178 & 20.3247 & 17.4833 \\
\hline NBG-17 & 18.5073 & 0.3617 & 1.95 & 18.5373 & 19.0877 & 17.8037 \\
\hline NBG-18 & 19.1966 & 0.3359 & 1.75 & 19.2517 & 19.8403 & 18.5113 \\
\hline PCEA & 20.9636 & 0.3885 & 1.85 & 20.9395 & 21.7497 & 20.0017 \\
\hline 2114 & 17.6985 & 0.1528 & 0.86 & 17.7063 & 17.9637 & 17.3549 \\
\hline $\begin{array}{c}\text { Against } \\
\text { Grain } \\
\text { Specimens }\end{array}$ & Mean & Std Dev & $\mathrm{CoV}(\%)$ & Median & $\begin{array}{c}\text { Upper } \\
\text { Limit }\end{array}$ & $\begin{array}{r}\text { Lower } \\
\text { Limit }\end{array}$ \\
\hline IG-110 & 19.2537 & 0.5447 & 2.83 & 19.1800 & 20.3247 & 18.5617 \\
\hline NBG-17 & 18.4601 & 0.4355 & 2.36 & 18.4492 & 19.0877 & 17.8037 \\
\hline NBG-18 & 19.4218 & 0.1930 & 0.99 & 19.4052 & 19.8403 & 19.1463 \\
\hline PCEA & 20.8375 & 0.2271 & 1.09 & 20.8537 & 21.3347 & 20.3340 \\
\hline 2114 & & & & & & \\
\hline $\begin{array}{l}\text { With Grain } \\
\text { Specimens }\end{array}$ & Mean & Std Dev & $\operatorname{CoV}(\%)$ & Median & $\begin{array}{c}\text { Upper } \\
\text { Limit }\end{array}$ & $\begin{array}{r}\text { Lower } \\
\text { Limit } \\
\end{array}$ \\
\hline IG-110 & 18.1879 & 0.4154 & 2.28 & 18.2137 & 19.0523 & 17.4833 \\
\hline NBG-17 & 18.5746 & 0.2096 & 1.13 & 18.5523 & 18.9147 & 18.1913 \\
\hline NBG-18 & 18.8462 & 0.1616 & 0.86 & 18.8990 & 19.0730 & 18.5113 \\
\hline PCEA & 21.0898 & 0.4722 & 2.24 & 21.1433 & 21.7497 & 19.9156 \\
\hline 2114 & 17.6985 & 0.1528 & 0.86 & 17.7063 & 17.9637 & 17.3549 \\
\hline
\end{tabular}


Appendix C Raw Data 


\section{References}

1. T. Burchell, R. Bratton, W. Windes, "NGNP Graphite Selection and Acquisition Strategy," ORNL/TM-2007/153, September 2007.

2. W. Windes, T. Burchell, R. Bratton, "Graphite Technology Development Plan," PLN-2497 Rev. 1, October 2010.

3. R. L. Bratton and T. D. Burchell, "NGNP Graphite Testing and Qualification Specimen Selection Strategy,” INL/EXT-05-00269, May 2005.

4. T. Burchell, J. Strizak, and M. Williams, "AGC-1 Specimen Preirradiation Data Report," ORNL/TM-2010/285, August 2011.

5. T. Reed, "AGC-1 Individual Specimen Fluence, Temperature, and Load Calculation and Tabulation," INL-ECAR-1943, September 2012.

6. T. Reed, AGC-1 As Run Thermal Results, INL-ECAR-1944, September 2012.

7. D. Swank, AGC-2 Graphite Preirradiation Data Package, INL/EXT-10-19588, August 2010.

8. M. Davenport, Technical and Functional Requirements, "Advanced Graphite Capsule AGC-3 Experiment Test Train,” TFR-791, June 2012.

9. DWG 601501, "ATR Advanced Graphite Capsule (AGC) AGC-3 Graphite Specimen Cutout Diagrams," July 11, 2012.

10. DWG 603520, “AGC-3 Test Train Facility Assembly,” June 26, 2012.

11. DWG 603521, AGC Graphite Specimen Holder Machining Details, June 14, 2012.

12. DWG 603524, AGC-3 Specimen Stack-up Arrangements, May 17, 2012.

13. J. R. Perry, "Thermal Evaluations for the AGC-3 Experiment Irradiated in the ATR East Flux Trap, TEM-10200-1, March 1, 2012.

14. T. Burchell and R. Bratton, "Graphite Irradiation Creep Capsule AGC-1 Experimental Plan, ORNL/TM-2005/505. May 2005.

15. T. Burchell, “A Revised AGC-1 Creep Capsule Layout, ORNL/TM-2009/009, January 2009.

16. DWG-601501, “AGC-3 Graphite Specimen Cutout Diagrams,” July 11, 2012.

17. R. D. Cowan, 1963, "Pulse Method of Measuring Thermal Diffusivity at High Temperatures," Journal of Applied Physics 34 (4): 926.

18. Y. S. Touloukian "Thermophysical Properties of Matter - Thermal Diffusivity," John Wiley \& Sons Ltd., May 6, 1973.

19. W. Windes et al., “AGC-2 Graphite Preirradiation Data Analysis Report INLEXT-13-28612,” April 2013. 